\title{
ON COMPLEX FERMI CURVES OF TWO-DIMENSIONAL PERIODIC SCHRÖDINGER OPERATORS
}

\author{
Inauguraldissertation \\ zur Erlangung des akademischen Grades \\ eines Doktors der Naturwissenschaften \\ der Universität Mannheim
}

vorgelegt von

Dipl.-Phys. Alexander Klauer

aus Heidelberg

Mannheim, 2011 
Dekan: $\quad$ Professor Dr. Wolfgang Effelsberg, Universität Mannheim Referent: $\quad$ Professor Dr. Martin Schmidt, Universität Mannheim Korreferent: Professor Dr. Andreas Knauf, Universität Erlangen-Nürnberg Tag der mündlichen Prüfung: 3. Juni 2011 


\begin{abstract}
In dimensions $d \geq 2$, the complex Bloch varieties and the associated Fermi curves of periodic Schrödinger operators with quasi-periodic boundary conditions are defined as complex analytic varieties. The Schrödinger potentials are taken from the Lebesgue space $L^{d / 2}$ in the case $d>2$, and from the Lorentz-Fourier space $\mathcal{F} \ell^{\infty, 1}$ in the case $d=2$. Then, an asymptotic analysis of the Fermi curves in the case $d=2$ is performed. The decomposition of a Fermi curve into a compact part, an asymptotically free part, and thin handles, is recovered as expected. Furthermore, it is shown that the set of potentials whose associated Fermi curve has finite geometric genus is a dense subset of $\mathcal{F} \ell^{\infty, 1}$. Moreover, the Fourier transforms of the potentials are locally isomorphic to perturbed Fourier transforms induced by the handles. Finally, an asymptotic family of parameters describing the sizes of the handles is introduced. These parameters are good candidates for describing the space of all Fermi curves.
\end{abstract}

\title{
Zusammenfassung
}

In $d \geq 2$ Dimensionen werden die komplexen Blochvarietäten und die zugehörigen Fermikurven periodischer Schrödingeroperatoren mit quasiperiodischen Randbedingungen als komplex analytische Varietäten definiert. Die Schrödingerpotentiale entstammen im Fall $d>2$ dem Lebesgueraum $L^{d / 2}$ und im Fall $d=2$ dem Lorentz-Fourier-Raum $\mathcal{F} \ell^{\infty, 1}$. Danach wird im Fall $d=2$ eine asymptotische Analyse der Fermikurven durchgeführt. Erwartungsgemäß erhält man die Aufteilung einer Fermikurve in einen kompakten Teil, einen asymptotisch freien Teil und dünne Henkel. Weiterhin wird gezeigt, dass die Menge der Potentiale, deren zugehörige Fermikurve endliches geometrisches Geschlecht hat, eine dichte Teilmenge von $\mathcal{F} \ell^{\infty, 1}$ ist. Überdies sind die Fouriertransformierten der Potentiale lokal isomorph zu von den Henkeln induzierten, gestörten Fouriertransformierten. Schließlich wird eine asymptotische Familie von Parametern, die die Größe der Henkel beschreiben, eingeführt. Diese Parameter sind gute Kandidaten, den Raum aller Fermikurven zu beschreiben. 



\section{Contents}

$\begin{array}{lll}1 & \text { Introduction } & 1\end{array}$

1.1 The inverse problem . . . . . . . . . . . . . . . . . 1

1.2 What is done in this work $\ldots \ldots \ldots \ldots \ldots$

1.3 The inverse problem in physics . . . . . . . . . . . . . 4

1.4 Acknowledgements $\ldots \ldots \ldots \ldots \ldots \ldots \ldots$

\begin{tabular}{|lll}
2 & Function spaces & 7
\end{tabular}

$2.1 \quad$ Measurable functions and Lebesgue spaces . . . . . . . . . . . 9

2.2 Sobolev spaces and potential spaces $\ldots \ldots \ldots \ldots . \ldots . \ldots$

2.3 Rearrangement-invariant function spaces . . . . . . . . . . . . . 22

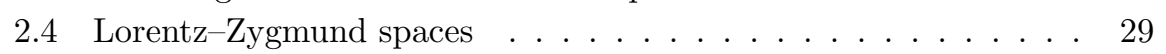

2.5 Fourier spaces $\ldots \ldots \ldots \ldots \ldots \ldots \ldots$

2.6 Bochner spaces and tensor products $\ldots \ldots \ldots \ldots$. . . . . . 38

2.7 Other spaces $\ldots \ldots \ldots \ldots \ldots \ldots \ldots$

2.7 .1 Hardy spaces . . . . . . . . . . . . . . . . . 44

2.7 .2 Banach manifolds . . . . . . . . . . . . . . . 47

2.8 Localisation of function space norms $\ldots \ldots \ldots \ldots \ldots$

2.8 .1 Localisation on the torus . . . . . . . . . . . . 47

2.8 .2 Localisation on discrete lattices . . . . . . . . . . . . 52

2.8 .3 Translation and levelling operators . . . . . . . . . 54

\begin{tabular}{lll}
\hline 3 & Schrödinger operators & 57
\end{tabular}

$3.1 \quad$ Schrödinger operators on the torus $\ldots \ldots \ldots \ldots$. . . . . 57

3.2 The resolvent of the free Schrödinger operator. . . . . . . . . . 59

3.3 Resolvents of general Schrödinger operators . . . . . . . . . . 65

$\begin{array}{lll}4 & \text { Fermi curves } & \mathbf{7 5}\end{array}$

4.1 Bloch varieties and Fermi curves $\ldots \ldots \ldots \ldots \ldots \ldots$

4.2 The free Fermi curve at $d=2 \ldots \ldots \ldots \ldots \ldots \ldots$

4.3 Asymptotic freeness $\ldots \ldots \ldots \ldots$

$4.4 \quad$ The constant potential Fermi curve at $d=2 \ldots \ldots \ldots$. . . 88

$4.5 \quad$ Asymptotic moduli parameterisation . . . . . . . . . . . . 91

$4.5 .1 \quad$ A nonlinear perturbation of the Fourier transform . . . . 91

$4.5 .2 \quad$ Approximation with potentials of finite type. . . . . . . . 104

$4.5 .3 \quad$ Per-double-point approximation of the Fermi curve . . . . 115 
$\begin{array}{llr}5 & \text { Summary and outlook } & \mathbf{1 2 5}\end{array}$

5.1 Works with different focus . . . . . . . . . . . . . . . . . . 125

5.2 Compact resolvent . . . . . . . . . . . . . . . . . . . 126

5.3 Fermi curve asymptotics . . . . . . . . . . . . . . . . . . . . . 129

5.4 Where to go from here . . . . . . . . . . . . . . . . . . . . . . . 129

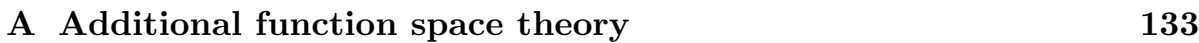

A.1 Lorentz-Karamata spaces . . . . . . . . . . . . . . . . . . 133

\begin{tabular}{|lr}
\hline B Failed extension to Dirac operators & 137
\end{tabular}

B.1 Dirac operators . . . . . . . . . . . . . . . . . . 138

B.1.1 Dirac operators on the torus . . . . . . . . . . . . . 138

B.1.2 Resolvents of free Dirac operators . . . . . . . . . . . . . 139

B.1.3 Resolvents of general Dirac operators . . . . . . . . . . . 142

B.2 Fermi curves of Dirac operators . . . . . . . . . . . . . . . . 145

B.2.1 Bloch varieties and Fermi curves . . . . . . . . . . . . . . 145

B.2.2 Free and asymptotically free Dirac Fermi curves . . . . . 148

B.3 Asymptotic analysis of Dirac Fermi curves . . . . . . . . . . . . . 150

B.3.1 A nonlinear perturbation of the Fourier transform . . . . 151

B.3.2 Approximation with finite-type Dirac Fermi curves . . . . 157

B.3.3 Failure to estimate the perturbed Fourier transforms . . . 159 


\section{List of Figures}

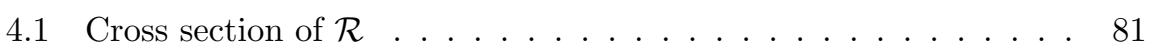

$8.2 \quad$ Cross section of $F(0) \ldots \ldots \ldots \ldots \ldots$

4.3 Two examples of handles $\ldots \ldots \ldots \ldots \ldots$ 


\section{Chapter 1}

\section{Introduction}

\subsection{The inverse problem}

The Schrödinger equation in $d$ dimensions, $d \in \mathbb{Z}_{>0}$, is given by

$$
(-\Delta+u) \psi=\lambda \psi
$$

Here, $u$ and $\psi$ are functions from $\mathbb{R}^{d}$ to $\mathbb{C}$, and $\lambda$ is a complex scalar. The function $u$ acts on $\psi$ by left multiplication. The Laplace operator $\Delta$, on the other hand, acts as the differential operator

$$
\Delta=\sum_{i=1}^{d} \frac{\partial^{2}}{\partial x_{i}^{2}}
$$

where $x_{1}, \ldots, x_{d}$ are coordinates for $\mathbb{R}^{d}$ with respect to the canonical ordered basis. Hence, for $d \geq 2$, the Schrödinger equation is a partial differential equation. We call the operator $-\Delta+u$ the Schrödinger operator with potential $u$. In particular, the Schrödinger equation is an eigenvalue equation for the Schrödinger operator in which a non-trivial solution $\psi$ is an eigenfunction belonging to the eigenvalue $\lambda$. The set of possible eigenvalues is the spectrum of the Schrödinger operator.

We are interested in periodic Schrödinger operators. By this, we mean two things.

Firstly, the potential $u$ must be a periodic function. For $d=1$, this condition has a very simple formalisation. Just pick some $b \in \mathbb{R}, b \neq 0$, and let $u$ fulfil the condition

$$
u(x+b)=u(x) \quad \text { for all } x \in \mathbb{R} .
$$

Of course, this condition does not in general remain the same if we pick a different $b$. In this sense, $b$ becomes a parameter for our locus of discourse. For general $d$, periodicity means not just one period, but $d$ independent periods. It is helpful to put slightly more sophistication into this concept than in the case $d=1$ and express periodicity through a geometric lattice $\Gamma \subseteq \mathbb{R}^{d}$ of rank $d$ :

$$
u(x+\gamma)=u(x) \quad \text { for all } x \in \mathbb{R}^{d} \text { and all } \gamma \in \Gamma .
$$


Secondly, a solution of the Schrödinger equation $\psi$ must be a quasi-periodic function, that is, there is some boundary condition $k \in \mathbb{C}^{d}$ such that

$$
\psi(x+\gamma)=e^{2 \pi i(k \mid \gamma)} \psi(x) \text { for all } x \in \mathbb{R}^{d}, \gamma \in \Gamma,
$$

where $(\cdot \mid \cdot)$ is the complex extension of the canonical Euclidean bilinear form on $\mathbb{R}^{d}$.

This second condition is not entirely independent of the first. The periodicity of $u$ and the chain rule imply

$$
(-\Delta+u(x)) \psi(x+\gamma)=((-\Delta+u) \psi)(x+\gamma) \quad \text { for all } x \in \mathbb{R}^{d}, \gamma \in \Gamma .
$$

In other words, the periodic Schrödinger operator commutes with all translation operators $T_{\gamma}, \gamma \in \Gamma$, which are defined by

$$
\left(T_{\gamma} \psi\right)(x):=\psi(x+\gamma)
$$

Hence, assuming the Schrödinger operator and the translation operators are diagonalisable in some suitable sense (take, for example, the Schrödinger operator as a closed operator on $L^{2}\left(\mathbb{R}^{d}\right)$ with real-valued potential), they are simultaneously so. Therefore, given some fixed solution $\psi$, there is a function $\chi: \Gamma \rightarrow \mathbb{C}$ such that the translation operators act on $\psi$ as

$$
T_{\gamma} \psi=\chi(\gamma) \psi \quad \text { for all } \gamma \in \Gamma
$$

in this case. The $\mathbb{Z}$-module structure of $\Gamma$ then yields

$$
\chi\left(\gamma_{1}+\gamma_{2}\right)=\chi\left(\gamma_{1}\right) \chi\left(\gamma_{2}\right) \text { for all } \gamma_{1}, \gamma_{2} \in \Gamma .
$$

Since the translation operators do not have zero eigenvalues, we arrive at 1.1.1), except that we posed this condition independently of the aforementioned prerequisites.

Although we introduced $k$ as a boundary condition, we shall consider it part of an extended spectrum of the Schrödinger operator. That is, for each Schrödinger operator (identified by its potential $u$ ) we define the Bloch variety

$$
\begin{aligned}
B(u):=\left\{(k, \lambda) \in \mathbb{C}^{d} \times \mathbb{C}: \text { There is a non-trivial solution } \psi\right. \text { of the } \\
\text { Schrödinger equation }(-\Delta+u) \psi=\lambda \psi \\
\text { such that } \left.\psi(x+\gamma)=\exp (2 \pi i(k \mid \gamma)) \psi(x) \text { for all } x \in \mathbb{R}^{d}, \gamma \in \Gamma\right\} .
\end{aligned}
$$

We can now pose the inverse problem of periodic Schrödinger operators. It consists of two subproblems.

- The moduli problem. Describe/parameterise the set of all Bloch varieties.

- The isospectral problem. Given a fixed potential $u_{0}$, describe/parameterise the set of all potentials $u$ such that $B(u)=B\left(u_{0}\right)$.

As it stands, the inverse problem is somewhat ill-posed. For example, what kind of "varieties" should the Bloch varieties be, and what space should we choose $u$ from such that $B(u)$ actually is such a variety? However, for the purposes of this introduction, we shall use this approximate problem specification, and provide the necessary additional details later in the work. 


\subsection{What is done in this work}

It turns out that a complete solution of the inverse problem is a very extensive programme. In this work, we shall solve only a small part of it. For $d=1$, the inverse problem has been solved thoroughly MO75, MM75, MT76, Kri77, MO80, Kor08. We shall specifically concentrate on the case $d=2$, for fixed $\lambda$. The latter condition means that we extract a fixed- $\lambda$ slice from the Bloch variety (called a Fermi curve). We then solve the moduli problem asymptotically, that is, for the large- $k$ case, for that slice. Let us explain what we do in the individual chapters.

In chapter 2, we introduce various function spaces, intended, amongst other things, for our potentials and solutions to live in. After all, we cannot expect the Bloch variety to have nice properties for just any distribution $u$. Nevertheless, we strive towards a space of potentials as large as possible to make the Bloch variety a complex variety. It turns out that for the case $d=2$, this requires a rather comprehensive collection of function spaces, including some less standard spaces. Besides the usual Lebesgue, Bessel potential, and Sobolev spaces, we shall also delve somewhat into the theory of rearrangement-invariant Banach function spaces, especially Lorentz-Zygmund spaces. We shall also deal with Fourier transforms and the spaces they define, as well as the duality of multiplication and convolution operators. In this context, we shall introduce some interpolation theory. Furthermore, we shall introduce Bochner spaces and investigate their relation to tensor products, and also mention some spaces of minor importance. We close the chapter with the introduction of several localisation techniques for function space norms. The vast majority of results presented in this chapter is well-known. Hence, the chapter should be thought of as a gentle exposition for beginners in the subject. Experts can probably skip the chapter on first reading.

In chapter 3, we execute the standard programme to establish the resolvent of the Schrödinger operator as a meromorphic, compact operator valued function on an $L^{2}$ Hilbert space. We also prove some continuity results with respect to different topologies. As we intend to do this for a relatively general space of potentials, we have to employ a small number of new techniques "under the hood" of the standard programme. Readers interested in these new techniques are advised to read the whole chapter, while others may content themselves with section 3.1 and theorem 3.3 .24 .

Chapter 4, is the heart of this work. With the results from chapter 3, we are finally able to give a proper definition of the Bloch variety and the Fermi curve. From then on restricting our attention to the case $d=2$, we embark on a systematic asymptotic analysis of the Fermi curve. First, we calculate the free $(u=0)$ Fermi curve explicitly, and then go on to show that general Fermi curves (or rather, certain reductions of them) consist of three parts: an asymptotically free part, an at most countable number of handles, and the remainder, which is contained in a compact set. The remainder of the chapter is concerned with the handles. Since constant potentials play a special role, we calculate the Fermi curves of constant potentials explicitly, and then define a special kind of Fourier transform of the potential, such that the resultant Fourier coefficients have a rather direct connexion with the geometry of the handles. This enables us to define potentials of finite type, and show that they are dense in our chosen space of potentials. Finally, we introduce a set of parameters describing the handles, thus solving the moduli problem for Fermi curves asymptotically. 
In chapter 5. we give a short summary of our results, discuss some results of others, and explain what further steps have to be taken to completely solve the entire inverse problem.

This work contains two appendix chapters. Appendix A is just a prequel to appendix B. In it, Lorentz-Karamata spaces, a generalisation of LorentzZygmund spaces due to Neves, are defined. This further scale of spaces is interesting in its own right as it has a certain completeness property (theorem A.1.6) which the other scales introduced up to that point lack. In appendix B we present the remnant of an attempt to achieve our results using Dirac operators instead of Schrödinger operators. Using Dirac theory instead of Schrödinger theory seemed quite feasible at first. The Dirac operator is simpler to handle than the Schrödinger operator, and in physics, the former is the relativistic version of the latter. Indeed, for fixed energy, the Dirac spectrum encompasses the Schrödinger spectrum. The further development may even work for $\mathrm{L}^{2}$ potentials. However, since we are using more general spaces of potentials, certain technicalities render the attempt doomed to fail. We have left it in, as far as it works, as an example that even a very beautiful and promising ansatz may, even after early successes, turn out to be unusable in the end.

\subsection{The inverse problem in physics}

Let us make some quick remarks about the connexion of the periodic Schrödinger operator and the inverse problem in general, with physics. This section highlights only a small number of examples. We make no claim of completeness.

The periodic Schrödinger operator has, at least in some cases, deep ties with physics. Indeed, Schrödinger himself was a physicist and used the Schrödinger equation to obtain the physical phenomenon of quantisation as an eigenvalue problem in 1926 Sch26b, Sch26d, Sch26a, Sch26c]. Already earlier, on the 21st of April, 1912, Friedrich and Knipping, on a proposal of Laue, demonstrated experimentally by scattering X-rays on a certain crystalline solid that the atoms in the solid are quite precisely arranged according to a three-dimensional lattice [MR87, fn. 316]. This leads to the assumption of a periodic effective electric potential in a crystalline solid 1 . Furthermore, the squared modulus of the eigenfunction, $|\psi|^{2}$, has a physical interpretation as observable probability density for a test particle (electron) in such a potential, giving rise to condition (1.1.1), at least for $k \in \mathbb{R}^{d}$. Therefore, the Schrödinger operator can serve as a model of physical reality for a broad class of scattering experiments.

The direct solution of the Schrödinger equation yields a spectrum for some concrete potential. A solution of the inverse problem, however, is of greater practical value, for the spectrum can be measured much more easily than the potential. For example, it is quite natural to ask what potentials can possibly be responsible for some measured spectrum (the isospectral problem). On the other hand, one might want to know whether one can create a physical configuration whose spectrum fulfils certain properties (the moduli problem).

This physical view of the inverse problem generalises beyond the Schrödinger operator, and is indeed older than the papers of Schrödinger. For example, H. Weyl writes in the introduction of Wey12 (a work dated May 7th, 1911):

\footnotetext{
${ }^{1}$ Several idealisations enter here, among them the neglection of thermally induced motion of the atoms and the finite size of the crystal.
} 
„Methoden von solcher Allgemeinheit wie die Theorie der Integralgleichungen haben, wenn man sie auf physikalische Probleme [...] anwendet, ihre Aufgabe damit nicht erschöpft, daß sie ermöglichen, in jedem konkreten Einzelfall die Erscheinungen bis in ihre letzten Details rechnerisch zu verfolgen [...]; vielmehr sollen diese Methoden [...] vor allem das leisten — was kein spezieller Ansatz zu leisten vermag -: die einem großen Komplex von Erscheinungen gemeinsamen Züge ausfindig zu machen. ${ }^{2} 2$

Weyl then goes on to show that for linear wave equations (in particular the case of electromagnetic waves trapped within a thrice differentiable, reflecting boundary), all spectra are asymptotically equal. This result can be seen as a partial solution to a moduli problem. Indeed, in chapter 4, we shall prove a similar statement for Fermi curves.

In any case, Weyl's "common traits" are not even restricted to one single kind of equation. In [GGKM67, Gardner, Greene, Kruskal, and Miura found a connexion between solitary water waves in shallow canals (which are described by the (non-linear) Korteweg-de Vries equation) and the Schrödinger equation. Their results in turn prompted new developments on the mathematical side (see section 5.1 for a selection of the outcomes).

In conclusion, we think it not unwise to view mathematics and physics going hand in hand as far as the theory is concerned, and thus we shall allow ourselves the occasional physical remark.

\subsection{Acknowledgements}

First, and foremost, I would like to thank my advisor, Prof. Martin Schmidt, for providing me with this research problem - especially after research in an earlier subject hit a dead end-and for the numerous conversations we had. Furthermore, I would like to thank my colleagues from our research group for a pleasant working atmosphere, and also all other folks from the A5 building I had the pleasure to lunch with.

On the private side, I would like to thank my parents for their continued support, my brother Andreas for an open ear, and Anke, even though she could not accompany the whole way. Last, but not least, I acknowledge the folks from the St. Ilgen/Brühl RPG groups as living proof that the world has other fun stuff besides mathematics.

\footnotetext{
2 "Methods of such generality as the theory of integral equations, when applied to physical problems, [...] do not exhaust their utility in making it possible to mathematically follow the phenomena of a concrete example to the last detail [...]; rather [...] these methods should provide for the following foremost — which no concrete ansatz can provide - : to identify the common traits in a grand complex of phenomena."
} 


\section{Chapter 2}

\section{Function spaces}

Setting out from the standard Lebesgue spaces we shall introduce all the function spaces necessary for our work.

The results presented in this chapter are, for the most part, well-known. Since the inception of Lebesgue integrals in 1901, a century of research has seen the introduction of multiple kinds of Banach function spaces ultimately derived from Lebesgue spaces. A considerable body of literature has emerged illuminating many important families of function spaces from many different angles. Hence, the greatest difficulty to the mere usufructuaries of what can arguably be called the cementing substance of modern functional analysis is not so much the adaptation and development of existing theory to their research specifics (for to be honest, some or other differential equation is often what is actually in the back of the function space creator's mind anyway) than, indeed, the choice of the right tool, which reduces, in effect, to the choice of the right (family of) function spaces. In the making of the present thesis a nontrivial amount of time was spent on research which family of function spaces might yield the desired results in best possible generality.

Naturally enough, we start with Lebesgue $L^{p}$-spaces of complex-valued functions defined on $\sigma$-finite measure spaces in section 2.1. In fact, the nature of periodic Schrödinger operators allows us to restrict our attention to but two kinds of measure spaces, namely flat $d$-dimensional tori and discrete lattices, each with their standard $\sigma$-algebras and measures.

Since the Schrödinger equation is a partial differential equation, the next logical step is to consider Sobolev $W^{m, p}$-spaces as a straightforward generalisation of the $L^{p}$-spaces. Indeed, there are certain Bessel potential spaces which are natural candidates for the target space of the free Schrödinger resolvent. These potential spaces can be identified with certain Sobolev spaces in most cases. But just in the case of dimension $d=2$, the case central to this work, this identification fails.

The failure of Sobolev spaces in the case $d=2$ is only marginal in terms of $L^{p}$-exponents. Therefore, we need a generalisation of $L^{p}$-spaces which allows for finer grained control over function regularity than just the exponent $p$. The resulting family of spaces should also be compatible with problems involving potentials. In particular, multiplication with a potential should be an operation as controllable as in the case of Lebesgue or Sobolev spaces. These properties are fulfilled by the rather general class of rearrangement-invariant Banach 
function spaces. Of course, derivatives are no longer explicit in the definitions of these spaces. Therefore, integral kernels of resolvent operators, which are handled rather implicitly by the Bessel and Sobolev machineries, must now be given explicit attention. As all occurring operators are translation invariant, the resulting integrals can be expressed as convolutions. Within certain bounds (see the remarks about Fourier spaces below) the convolution operation can be considered dual to the multiplication operation. As long as this duality is valid, the use of convolutions does not pose any additional difficulties.

The most significant shortcoming of rearrangement-invariant Banach function spaces, however, is that in their (local or global) regularity properties, they are constrained by $L^{1}$ - and $L^{\infty}$-spaces. For example, while a sufficiently regular Sobolev space can easily escape the scale ${ }^{1}$ of Lebesgue spaces and enter the scale of continuous or even Hölder continuous functions, a rearrangementinvariant Banach function space can never escape the bounds set by $L^{1}$ and $L^{\infty}$. Even within these bounds, limits of this type sometimes occur on subscales of Banach function spaces. However, it will be desired to escape these limits in at least one case. The solution is to drop consideration of Banach function spaces in favour of quasi-Banach function spaces by requiring only a weak form of the triangle inequality.

Lorentz-Zygmund spaces are a subclass of rearrangement-invariant quasiBanach function spaces amenable to formulation of user-friendly interpolation results on the one hand, and explicitly containing the Lebesgue $L^{p}$-spaces as a special case on the other. In a greater part of this work, we shall almost exclusively operate in this family of spaces.

It will turn out that instead working in location space (the compact torus), a larger class of potentials can be treated by a Fourier transform of the whole setting to momentum space (a discrete momentum lattice). This necessitates the introduction of Fourier spaces, i.e. spaces of functions (or, rather, distributions) whose Fourier transforms belong to certain Lorentz-Zygmund spaces. Such a transformation adds a layer of indirection to the problem: clearly, a space of functions whose Fourier transforms have certain properties is less concrete than a space of functions endowed with the same such properties themselves. It is therefore appropriate to question whether the gain in generality satisfies the accompanying loss in concreteness. Luckily, in our case the results from location space theory combined with the well-understood interpolation properties of the Fourier transform near $L^{1}$ with respect to Lorentz-Zygmund spaces thoroughly alleviate this problem, for they enable us to develop the concrete, less general version of our theorems alongside the general ones.

Occasionally, we shall encounter multivariate functions. By currying, i.e. "domain and codomain shuffling", these functions may be considered as univariate with values in function spaces. As such, they become amenable to Bochner integration theory. In particular, one can define (quasi-)Banach function spaces of multivariate functions using Bochner integrals in the usual definition of Lebesgue spaces. It is possible, in principle, to generalise this scheme to the case of Lorentz-Zygmund spaces. We shall, however, take a slightly different route and start with the representability of Bochner spaces which are based on separable Lebesgue spaces as tensor products of such spaces. The tensor

\footnotetext{
${ }^{1} \mathrm{~A}$ scale of spaces in the informal sense is best imagined as a family of spaces indexed by one or more variables running through subintervals of $[-\infty, \infty]$. Attempts to formalise the concept of a scale have been made, see e.g. KP66.
} 
product representation, despite being formally equivalent to the Bochner space representation in many cases, eases the handling of linear operators between Bochner spaces considerably. Generalisation to the Lorentz-Zygmund case is straightforward. What remains is the rather subtle question under what circumstances boundedness of linear operators is hereditary with respect to the tensor product operation. It turns out that multiplication and convolution are compatible while the Fourier transform is not. This demonstrates another advantage of using Fourier spaces, as we need to take tensor products only of functions which have already been Fourier transformed.

This completes the collection of linear function spaces we work extensively with. At this point we slide in a small section on two less extensively used structures. Earlier, we had mentioned the failure of equivalence of Sobolev and Bessel potential spaces in certain corner cases. In the case of the free Schrödinger equation the best possible replacement in one such case for the Lebesgue space $L^{1}$ is the real Hardy space. Unfortunately, the real Hardy space exhibits some properties which must be considered pathological when it comes to multiplication with a function. Nevertheless, the fact that the real Hardy space is the optimal replacement for $L^{1}$ in the free case gives important clues as to which rearrangement-invariant function space is optimal in the non-free case. Lastly, we shall recall the definition of a Banach manifold, which is the correct structure to encode the set of Fermi curves.

In the last section of this chapter we introduce two processes by which function space norms can be localised. When estimating the bound of a linear operator the only question of interest is usually whether the bound is finite or infinite. The one important exception is when, for example in the creation of an inverse, the operator is used in the definition of an infinite series such as a Neumann series. In this case, a more explicit bound is required, usually smaller than one. The actual bound of a linear operator is malleable by replacing the norms of its domain and codomain with equivalent norms or quasi-norms. Localisation is just such a process. We shall employ localisation on finite and on discrete measure spaces. Expectably, localisation is only applicable to a single linear operator, or a small neighbourhood of a single linear operator of a certain class. It is, in general, not uniform. The failure of uniformness provides an insight into the internal structure of the relevant spaces.

\subsection{Measurable functions and Lebesgue spaces}

Let $(X, \Sigma, \mu)$ be a measure space (see Bor98 $^{2}$ for the modern concept of measure; a contemporary survey of pre-Borelian attempts can be found in Sch00, II.4]). Furthermore, let $\overline{\mathbb{C}}:=\mathbb{C} \cup\{\infty\}$ with the Riemann sphere standard topology. Now, let $\mathfrak{M}((X, \Sigma, \mu), \overline{\mathbb{C}})$ be the set of all measurable, essentially finitevalued extended complex-valued functions on $X$, that is all functions $f: X \rightarrow \overline{\mathbb{C}}$ $\mu$-almost all values of which are finite, and for which $f^{-1}(U) \in \Sigma$ whenever $U$ is open. As there will be no opportunity for confusion, we shall simply write $\mathfrak{M}(X, \overline{\mathbb{C}})$ for this set.

Sometimes we only consider functions with values in $[0, \infty]$, giving rise to

\footnotetext{
${ }^{2}$ The author found a yellowed and heavily sunned copy of the fourth (1950) edition of this work in the department library. Its pages were still uncut! Thus decades worth of students must have been prevented from perusing this original text on measure theory.
} 
the set $\mathfrak{M}(X,[0, \infty])$, where we endow $[0, \infty]$ with the relative topology. As a further abbreviation of notation, we shall write $\mathfrak{M}(X)$ for $\mathfrak{M}(X, \overline{\mathbb{C}})$ and $\mathfrak{M}^{+}(X)$ for $\mathfrak{M}(X,[0, \infty])$.

We shall formulate our statements for generic $\sigma$-finite measure spaces $X$ whenever it is not inconvenient. However, it will often be more convenient to work directly with the measure spaces inherent to the problem at hand. Therefore we shall introduce right here the two measure spaces used most commonly in this work.

Let $\mathbb{R}^{d}$ be the standard $d$-dimensional real vector space. Unless we say so otherwise, we shall assume $d \geq 2$. Endowed with the standard Euclidean bilinear form $(\cdot \mid \cdot)$ with respect to its canonical basis, this space becomes the standard $d$ dimensional real geometric vector space. Moreover, it becomes a measure space with respect to the Lebesgue measure Leb02.

Now, let $B$ be an arbitrary, ordered basis of $\mathbb{R}^{d}$ and let $\Gamma$ be the free $\mathbb{Z}$ module over $B$. The embedding $\mathbb{Z} \subseteq \mathbb{R}$ induces a unique embedding $\Gamma \subseteq$ $\mathbb{R}^{d}$. Thus $\Gamma$ inherits the symmetric bilinear form $(\cdot \mid \cdot)$ from $\mathbb{R}^{d}$ and becomes a (geometric) lattice. The concept of a lattice is an obvious abstraction from structures appearing in nature (e.g. in crystallography) and has found its way into modern mathematics already in the 19th century, if not earlier. A recent account of lattice theory can be found in CS88.

The first measure space we shall need regularly is the flat torus $F:=\mathbb{R}^{d} / \Gamma$. The torus $F$ may also be identified with a fundamental domain in $\mathbb{R}^{d}$ and thus $F$ inherits its $\sigma$-algebra and its measure from the Lebesgue measure on $\mathbb{R}^{d}$. The boundary of the fundamental domain is immaterial in this context. Also note that without taking the basis $B$, from which $\Gamma$ was originally constructed, into account, the choice of a fundamental domain may not be unique, even modulo translations. The resulting measure on $F$, however, is. The most important additional property of $F$ is that, since $B$ is a generating set, it is a finite measure space.

Each lattice $\Gamma$ has its $\mathbb{Z}$-module of linear forms $\Gamma \rightarrow \mathbb{Z}$. The canonical vector space isomorphism $\operatorname{Hom}\left(\mathbb{R}^{d}, \mathbb{R}\right) \cong \mathbb{R}^{d}$ induces the representation

$$
\Gamma^{*}:=\left\{x \in \mathbb{R}^{n}:(x \mid \gamma) \in \mathbb{Z} \text { for all } \gamma \in \Gamma\right\}
$$

for this structure. Clearly, $\Gamma^{*}$ is a lattice. It is called the dual lattice to $\Gamma$. The dual lattice arises naturally when Fourier-transforming a function on $F$. Hence, $\Gamma^{*}$ shall be our second common measure space. Since $\Gamma^{*}$ is a discrete space, we naturally use a counting measure on $\Gamma^{*}$ such that $\Gamma^{*}$ is a completely atomic measure space, with each atom having measure 1.

In this work, we shall encounter the results of developments and variations on the general theme of normed spaces, which revolve around a functional $\|\cdot\|: V \rightarrow$ $\mathbb{C}$ on a complex vector space $V$. We begin with the enumeration of the most common properties normally required of such a functional Ban22:

(F1) Positivity: $\|x\| \geq 0$ for all $x \in V$ (in particular, $\|x\| \in \mathbb{R}$ ).

(F2) Regularity: If $x \in V$, then $\|x\|=0$ if and only if $x=0$.

(F3) Absolute linearity: $\|\lambda x\|=|\lambda|\|x\|$ for all $\lambda \in \mathbb{C}$ and for all $x \in V$.

(F4) Triangle inequality: $\|x+y\| \leq\|x\|+\|y\|$ for all $x, y \in V$. 
(F5) Completeness: If $\left(x_{n}\right)_{n \in \mathbb{N}}$ is a sequence with $x_{n} \in V$ for all $n \in \mathbb{N}$ and $\lim _{n, m \rightarrow \infty}\left\|x_{n}-x_{m}\right\|=0$, then $\left(x_{n}\right)_{n \in \mathbb{N}}$ has a limit $x \in V$, i.e. there is an $x \in V$ such that $\lim _{n \rightarrow \infty}\left\|x_{n}-x\right\|=0$.

If $\|\cdot\|$ fulfils (F1), (F3) and (F4), it is called a semi-norm. If, in addition, (F2) is fulfilled, it is a norm. Finally, if $\|\cdot\|$ satisfies (F1) through (F5), $(V,\|\cdot\|)$ is called a complex Banach space. Again, we shall write $V$ instead of $(V,\|\cdot\|)$ if no confusion is possible. Remember that the limit in (F5) may not be unique without (F2).

The complex numbers $\mathbb{C}$ constitute a valued field which is complete with respect to its valuation and thus is certainly a Banach space. This property extends to certain subsets of $\mathfrak{M}(X)$. The Lebesgue integral Leb01 allows the definition of the functional

$$
\|f\|_{p}:= \begin{cases}\left(\int_{X}|f|^{p} \mathrm{~d} \mu\right)^{1 / p} & (0<p<\infty), \\ \inf _{N \in \Sigma} \sup _{\mu(N)=0} \mid f \in X \backslash N & (p=\infty),\end{cases}
$$

leading to the definition of the spaces

$$
\widetilde{L}^{p}(X, \mathbb{C}):=\left\{f \in \mathfrak{M}(X):\|f\|_{p}<\infty\right\} .
$$

Note that we omit the bar over $\mathbb{C}$ even though the functions in $\widetilde{L}^{p}(X, \mathbb{C})$ may have singularities. Obviously, $\|\cdot\|_{p}$ fulfils (F1) and (F3). Generalising a convexity theorem of Minkowski [Min96, IV.40] to Lebesgue integrals yields (F4) provided that $p \geq 1$. Hence, in the case of $\|\cdot\|_{p},(\mathrm{~F} 4)$ is also called Minkowski's inequality. While Minkowski proved his inequality using partial derivatives and case elimination, modern treatments often hide the convexity information in the more basic Young's inequality. The proof Minkowski's inequality with Young's inequality makes use of another Lebesgue adapted variation of an inequality, which is due to L. J. Rogers Rog87, this time regarding mean values for products. Nowadays, this inequality bears the name Hölder's inequality as Hölder generalised Rogers's result Hö89. Ironically, Rogers's less general version is all what would have been necessary for F. Riesz to state Hölder's inequality in its modern form Rie10, $\$ 2]^{3}$

Theorem 2.1.1 (Hölder's inequality for $p$-integrable functions). Given $0<$ $p, q, r \leq \infty$ with $r^{-1}=p^{-1}+q^{-1}$, the inequality

$$
\|f g\|_{r} \leq\|f\|_{p}\|g\|_{q}
$$

holds for all $f, g \in \mathfrak{M}(X)$ whenever the multiplications make sense.

Riesz stated this inequality only for $r=1$ but, being a multiplicative inequality, it readily generalises to all positive $r$ by using $|f|^{r}$ and $|g|^{r}$ in place of $f$ and $g$.

The spaces $\widetilde{L}^{p}(X, \mathbb{C})$ also fulfil the completeness property (F5), even for $p<$ 1 [Rie09], a result which bears the name of Fischer and F. Riesz as, after a hint from Hilbert, these two authors arrived independently at a preliminary result

\footnotetext{
${ }^{3}$ Riesz attributed the inequality to Cauchy $(p=2)$ and Hölder, omitting Rogers. On the other hand, he correctly attributed (F4) to Minkowski. However, he suggested Minkowski proved his inequality in 1907 , when in fact he did so as early as 1896 .
} 
concerning Fourier transforms of square-integrable functions Rie07b, Rie07a, Fis07.

However, the spaces $\widetilde{L}^{p}(X, \mathbb{C})$ do not necessarily fulfil the regularity property (F2). If $N \in \Sigma$ is a non-empty set of zero measure, then the characteristic function $\chi_{N}$ is nonzero, but $\left\|\chi_{N}\right\|_{p}=0$. So $\widetilde{L}^{p}(X, \mathbb{C})$ are not Banach spaces whenever a set such as $N$ exists in $X$. The simple solution is to divide out the kernel of zero measure sets:

$$
L^{p}(X, \mathbb{C}):=\widetilde{L}^{p}(X, \mathbb{C}) /\left\{f \in \widetilde{L}^{p}(X, \mathbb{C}):\|f\|_{p}=0\right\} .
$$

This operation does not hurt the other properties (F1), (F3), (F4) and (F5) and clearly the functional $\|\cdot\|_{p}$ is well-defined on $L^{p}(X, \mathbb{C})$. Hence, $L^{p}(X, \mathbb{C})$ is a Banach space for $1 \leq p \leq \infty$. We shall write $L^{p}(X)$ as a shorthand for $L^{p}(X, \mathbb{C})$, and $\ell^{p}\left(\Gamma^{*}\right)$ for $L^{p}\left(\Gamma^{*}\right)$. Moreover, we will not explicitly distinguish between measurable functions and equivalence classes of such functions with respect to the above kernel.

In the remainder of this section, we shall investigate how the $L^{p}$-spaces are interrelated to each other. As $\|\cdot\|_{p}$ is defined via an integral (at least for $p<\infty$, but the case $p=\infty$ is usually easier anyway), its values are influenced by both the local (e.g. discontinuities and singularities) and the global (e.g. decay behaviour) structure of its arguments. Therefore interrelation analysis is usually done in terms of sums and intersections of $L^{p}$-spaces with mutually dual exponents. In order to simplify things, however, we shall do this analysis for $X=F$ and $X=\Gamma^{*}$ separately, where there are no global and no local contributions, respectively.

Proposition 2.1.3. Let $0<p \leq q \leq \infty$, then $L^{q}(F) \subseteq L^{p}(F)$ and the embedding is continuous.

Proof. Since $\mu(F)<\infty,\|1\|_{r}=\left\|\chi_{F}\right\|_{r}<\infty$ for all $0<r \leq \infty$. Hence, the proposition follows immediately from Theorem 2.1.1.

In the case $X=\Gamma^{*}$ it is quite the other way round:

Proposition 2.1.4. Let $0<p \leq q \leq \infty$, then $\ell^{p}\left(\Gamma^{*}\right) \subseteq \ell^{q}\left(\Gamma^{*}\right)$ and the embedding is continuous.

Proof. All but a finite number of values of a $p$-summable function on $\Gamma^{*}$ must have modulus smaller than one, so the inclusion is obvious. Continuity follows from general considerations regarding this type of space, see for example BS88. Theorem 1.1.8].

With the inclusion relations among the spaces $L^{p}(F)$ and $\ell^{p}\left(\Gamma^{*}\right)$, respectively, cleared up, we now turn to the matter of the relations between these two classes of spaces. As we have mentioned before, the lattice $\Gamma^{*}$ is the natural dual partner of $F$. As an illustration, define

$$
\begin{aligned}
\psi_{\kappa}: \mathbb{R}^{d} & \rightarrow \mathbb{C}, \\
x & \mapsto e^{2 \pi i(\kappa \mid x)}
\end{aligned}
$$

for $\kappa \in \mathbb{C}^{d}$. Clearly, if $\kappa \in \Gamma^{*}$, then $\psi_{\kappa}$ is $\Gamma$-periodic. Therefore, we shall take $F$ as the domain of $\psi_{\kappa}$ in this case. This is a family of uniformly bounded, 
infinitely differentiable functions. The duality between $F$ and $\Gamma^{*}$ extends, to a limited degree, to the Lebesgue spaces. We can define the Fourier transform via the Fourier integral

$$
(\mathcal{F} f)(\kappa):=\int_{F} \psi_{-\kappa}(x) f(x) \mathrm{d} x
$$

We also write $\widehat{f}$ for $\mathcal{F} f$.

We need to make precise relations between domains and codomains of $\mathcal{F}$. This is best done in the setting of distribution theory as begun by Sobolev in Sob63 and, independently, L. Schwartz, whose life work consists of work related to distribution theory to a good part. We shall not go into too much detail and only mention the key results without proof. Let us begin with two benign metric spaces. In what follows, a multi-index will always be a $d$-tuple $\alpha \in\left(\mathbb{Z}_{>0}\right)^{d}$ and $|\alpha|$ will denote the 1-norm of $\alpha$. We also assume the reader knows the standard multi-index operations.

Definition 2.1.5. We define the Schwartz function spact 4

$$
\mathfrak{S}(F):=C^{\infty}(F)
$$

where $C^{\infty}(F)$ is the space of infinitely real differentiable functions on $F$, and the Schwartz sequence space

$$
\mathfrak{s}\left(\Gamma^{*}\right):=\left\{f: \Gamma^{*} \rightarrow \mathbb{C}: \sup _{\kappa \in \Gamma^{*}}\left(1+\kappa^{2}\right)^{n / 2}|f(\kappa)|<\infty \text { for all } n \in \mathbb{N}\right\} .
$$

Here, $\kappa^{2}$ is just a shorthand for $(\kappa \mid \kappa)$. We topologise these two spaces by defining two families of Fréchet semi-norms, $\left\{P_{n}\right\}_{n \in \mathbb{N}}$ for $\mathfrak{S}(F)$, and $\left\{p_{n}\right\}_{n \in \mathbb{N}}$ for $\mathfrak{s}\left(\Gamma^{*}\right)$, by

$$
\begin{aligned}
& P_{n}(f):=\sup _{x \in F} \sum_{|\alpha| \leq n}\left|\left(\partial^{\alpha} f\right)(x)\right|, \\
& p_{n}(f):=\sup _{\kappa \in \Gamma^{*}}\left(1+\kappa^{2}\right)^{n / 2}|f(\kappa)|,
\end{aligned}
$$

where $\alpha$ is a multi-index, yielding the metric

$$
d(f, g):=\sum_{n \in \mathbb{N}} 2^{-n} \frac{P_{n}(f-g)}{1+P_{n}(f-g)}
$$

on $\mathfrak{S}(F)$, and likewise on $\mathfrak{s}\left(\Gamma^{*}\right)$.

Clearly, the Fourier transform is defined for all $f \in \mathfrak{S}(F)$. We also have

Proposition 2.1.6. Let $f \in \mathfrak{S}(F)$ and $\alpha$ be a multi-index. Then

$$
\widehat{\partial^{\alpha} f}=(2 \pi i \kappa)^{\alpha} \widehat{f} .
$$

\footnotetext{
${ }^{4}$ On an Euclidean space, one would define the Schwartz function space to be the rapidly decreasing smooth functions. Since $F$ is compact, this is of course unnecessary here.
} 
Given this proposition, we see that $\mathcal{F}$ maps $\mathfrak{S}(F)$ continuously into $\mathfrak{s}\left(\Gamma^{*}\right)$. Like the Fourier integral for functions in $\mathfrak{S}(F)$, we can define the Fourier series for sequences in $\mathfrak{s}\left(\Gamma^{*}\right)$ :

$$
\left(\mathcal{F}^{\prime} f\right)(x):=\frac{1}{\mu(F)} \sum_{\kappa \in \Gamma^{*}} \psi_{\kappa}(x) f(\kappa) .
$$

We have the following central theorem:

Theorem 2.1.8 (Fourier inversion theorem). The Fourier transform $\mathcal{F}$ is a homeomorphism from $\mathfrak{S}(F)$ to $\mathfrak{s}\left(\Gamma^{*}\right)$, and its inverse is $\mathcal{F}^{\prime}$.

Due to this theorem we sometimes use $\mathcal{F}$ instead of $\mathcal{F}^{\prime}$ when no confusion is possible. We also mark Fourier transformations in both directions by $\widehat{\imath}$. We now define the following important operation:

Definition 2.1.9 (convolution). For $f, g \in \mathfrak{S}(F)$, we define the convolution

$$
(f * g)(x):=\int_{F} f(x-y) g(y) \mathrm{d} y,
$$

and likewise for $f, g \in \mathfrak{s}\left(\Gamma^{*}\right)$ :

$$
(f * g)(\kappa):=\sum_{\lambda \in \Gamma^{*}} f(\kappa-\lambda) g(\lambda)
$$

The convolution is a bilinear operator on $\mathfrak{S}(F)$ and $\mathfrak{s}\left(\Gamma^{*}\right)$, respectively. It is the dual operation to multiplication in the following sense:

Theorem 2.1.10 (convolution theorem). Let $f_{1}, g_{1} \in \mathfrak{S}(F)$ and $f_{2}, g_{2} \in \mathfrak{s}\left(\Gamma^{*}\right)$, then

$$
\begin{array}{ll}
\widehat{f_{1} * g_{1}}=\widehat{f}_{1} \widehat{g}_{1}, & \widehat{f_{1} g_{1}}=\frac{1}{\mu(F)}\left(\widehat{f}_{1} * \widehat{g}_{1}\right), \\
\widehat{f_{2} * g_{2}}=\mu(F) \widehat{f}_{2} \widehat{g}_{2}, & \widehat{f_{2} g_{2}}=\widehat{f}_{2} * \widehat{g}_{2} .
\end{array}
$$

We can greatly expand the scope of the Fourier transform and the convolution by the following means.

Definition 2.1.12 (tempered distributions). Let $\mathfrak{S}^{*}(F)$ the space of continuous linear functionals on $\mathfrak{S}(F)$. We endow this space with the weak ${ }^{*}$ topology and call its elements the tempered distributions. We define the Fourier transform of $\omega \in \mathfrak{S}^{*}(F)$ to a sequence on $\Gamma^{*}$ by

$$
(\mathcal{F} \omega)(\kappa):=\omega\left(\psi_{-\kappa}\right)
$$

(recall that $\psi_{\kappa} \in \mathfrak{S}(F)$ for all $\kappa \in \Gamma^{*}$ ).

The next proposition makes the relevance of this definition obvious.

Proposition 2.1.14. For each $f \in \mathfrak{S}(F)$ the function

$$
\begin{aligned}
\omega_{f}: \mathfrak{S}(F) & \rightarrow \mathbb{C}, \\
g & \mapsto \int_{F} f(x) g(x) \mathrm{d} x
\end{aligned}
$$


is an element of $\mathfrak{S}^{*}(F)$. The mapping $f \mapsto \omega_{f}$ is continuous, injective, has a dense image, and in particular

$$
\widehat{f}=\mathcal{F} \omega_{f}
$$

for all $f \in \mathfrak{S}(F)$.

This proposition allows us to concretise the "abstract" Fourier transform defined in (2.1.13) for all kinds of objects for which a construction like 2.1.15) makes sense. More generally, the embedding $\mathfrak{S}(F) \hookrightarrow \mathfrak{S}^{*}(F)$ induced by this definition allows a domain enlargement of the Fourier integral for locally convex spaces through Hahn-Banach extensions [Hel12. We can now deal with the codomain:

Theorem 2.1.16 (extension of Fourier inversion). Define the space $\mathfrak{s}^{*}\left(\Gamma^{*}\right)$ by

$$
\begin{array}{r}
\mathfrak{s}^{*}\left(\Gamma^{*}\right):=\left\{f: \Gamma^{*} \rightarrow \mathbb{C}: \text { There is a } C>0 \text { and an } n \in \mathbb{N}\right. \\
\text { such that } \left.|f(\kappa)| \leq C\left(1+\kappa^{2}\right)^{n / 2} \text { for all } \kappa \in \Gamma^{*}\right\} .
\end{array}
$$

Let $f \in \mathfrak{s}^{*}\left(\Gamma^{*}\right)$, then for every $g \in \mathfrak{s}\left(\Gamma^{*}\right)$ the product $f g$ is summable. Moreover, the linear functional

$$
\begin{aligned}
\omega_{f}: \mathfrak{s}\left(\Gamma^{*}\right) & \rightarrow \mathbb{C}, \\
g & \mapsto \sum_{\kappa \in \Gamma^{*}} f(\kappa) g(\kappa)
\end{aligned}
$$

is continuous. The mapping $f \mapsto \omega_{f}$ is a bijection from $\mathfrak{s}^{*}\left(\Gamma^{*}\right)$ to the space of continuous linear functionals on $\mathfrak{s}\left(\Gamma^{*}\right)$. In particular, $\mathfrak{s}^{*}\left(\Gamma^{*}\right)$ inherits the weak ${ }^{*}$ topology. The Fourier transform is a homeomorphism from $\mathfrak{S}^{*}(F)$ to $\mathfrak{s}^{*}\left(\Gamma^{*}\right)$. Its inverse $\mathcal{F}^{\prime}$ is given by

$$
\left(\mathcal{F}^{\prime} f\right)(g)=\frac{1}{\mu(F)} \sum_{\kappa \in \Gamma^{*}} f(\kappa) \widehat{g}(-\kappa) .
$$

In particular, $\mathfrak{s}\left(\Gamma^{*}\right) \subseteq \mathfrak{s}^{*}\left(\Gamma^{*}\right)$ densely and

$$
\mathcal{F}^{\prime} f=\omega_{\widehat{f}}
$$

for all $f \in \mathfrak{s}\left(\Gamma^{*}\right)$, with $\omega_{\widehat{f}}$ as in 2.1.15.

We first use this theorem to extend the convolution from $\mathfrak{S}(F)$ to $\mathfrak{S}^{*}(F)$. Clearly, $\mathfrak{s}^{*}\left(\Gamma^{*}\right)$ is closed under multiplication, and multiplication is continuous on this space. In light of the convolution theorem 2.1.10, this allows us to define

$$
\omega * \chi:=\mathcal{F}^{\prime}((\mathcal{F} \omega)(\mathcal{F} \chi))
$$

for $\omega, \chi \in \mathfrak{S}^{*}(F)$. In particular, the first and the last equation of 2.1.11 generalise to $f_{1}, g_{1} \in \mathfrak{S}^{*}(F)$ and $f_{2}, g_{2} \in \mathfrak{s}^{*}\left(\Gamma^{*}\right)$. The remaining two equations, however, do not. Indeed, it is generally impossible to multiply two distributions from $\mathfrak{S}^{*}(F)$, and, likewise, convolute two distributions from $\mathfrak{s}^{*}\left(\Gamma^{*}\right)$. However, some less general extensions certainly do exist, which brings us back to our actual objects of study, the Lebesgue spaces. 
The identification 2.1.15 makes sense for $f \in L^{1}(F)$ and hence, by Proposition 2.1.3. for all $f \in L^{p}(F), 1 \leq p \leq \infty$. We also clearly have $\ell^{p}\left(\Gamma^{*}\right) \subseteq \mathfrak{s}^{*}\left(\Gamma^{*}\right)$ for $0<p \leq \infty$, yielding a natural definition of the Fourier transform on these Lebesgue spaces. As for a suitable codomain, $\mathcal{F}$ maps $L^{1}(F)$ continuously into $\ell^{\infty}\left(\Gamma^{*}\right)$ and $\ell^{1}\left(\Gamma^{*}\right)$ continuously into $L^{\infty}(F)$ by the triangle inequality. The counterpoint is provided by Parseval's theorem which stipulates that $L^{2}(F)$ and $\ell^{2}\left(\Gamma^{*}\right)$ are isomorphic with the Fourier transforms as isomorphisms. This theorem has found widespread use not only in mathematics but also in physics and engineering. There are claims that Parseval actually omitted the proof of his theorem ${ }^{5}$ but the need to prove this important identity in a modern setting was clearly recognised. For example, the paper Ray02 of Rayleigh contains what is essentially a proof for one-dimensional Gaussians, but the methodology can (perhaps with a little hindsight) be expanded to much more general classes of functions. Nowadays, Parseval's theorem is seen as a special case of Plancherel's theorem Pla10, which works in all locally compact vector spaces.

The usual shorthand to describe the behaviour of the Fourier operators expounded in the previous paragraph is to say they are of (strong) types $(1, \infty)$ and $(2,2)$. More generally, any operator is said to be of type $(p, q)$, if it maps a Lebesgue space with exponent $p$ continuously into a Lebesgue space with exponent $q$. The domain (i.e. whether we have an $L^{p}$ - or an $\ell^{p}$-space) is omitted when confusion is not a concern. More general results of the form $\left(2^{n} /\left(2^{n}-1\right), 2^{n}\right)$ $\left(n \in \mathbb{Z}_{>0}\right)$ for the Fourier operator due to W. H. Young You12b, You13] led to the following interpolation result by Hausdorff [Hau23].

Theorem 2.1.17 (Hausdorff-Young inequality). Let $1 \leq p \leq 2$ and $2 \leq q \leq \infty$ such that $p^{-1}+q^{-1}=1$. Then the Fourier transforms are of type $(p, q)$.

This theorem provides a device to change between Lebesgue spaces $L^{p}$ and $\ell^{q}$ with important restrictions on $p$ and $q$ as stated.

We remark that interpolation results such as the previous theorem can be acquired for general operators. This theory has been started by M. Riesz [Rie27]. The actual connexion to usual interpolation (convex combination of inverses of Lebesgue exponents) was made by Thorin [Tho39], leading to the name RieszThorin interpolation. Interpolation will play an crucial role in our work, but we shall base its introduction in section 2.3 on a different approach due to Marcinkiewicz in combination with an extension of Thorin interpolations due to Calderón.

With the convolution as the natural counterpart of pointwise multiplication, we need a counterpart to Hölder's inequality in Lebesgue spaces. The following inequality holds:

Theorem 2.1.18 (Young's inequality for $p$-integrable functions You12a]). Let $1 \leq p, q, r \leq \infty$ with $r^{-1}=p^{-1}+q^{-1}-1 \geq 0$. Then

$$
\|f * g\|_{r} \leq\|f\|_{p}\|g\|_{q}
$$

for all $f, g \in \mathfrak{M}(X)$ whenever the inequality makes sense.

Multiplication, convolution and the Fourier transforms will play an important role in other Banach spaces of functions, too. Therefore we shall generalise some of the results of this section in the following sections.

\footnotetext{
${ }^{5}$ Parseval's original treatment [Par06] has recently been made available on the web. The author encourages interested readers to verify these claims themselves.
} 


\subsection{Sobolev spaces and potential spaces}

Sobolev spaces are spaces of $L^{p}$-functions certain derivatives of which are also of $L^{p}$ type, with the same $p$. We shall need Sobolev spaces only over the compact torus $F$. Hence, the easiest way for us to arrive at a precise definition of Sobolev spaces is the strong extension method. The space $C^{0}(F)$, that is, the space of continuous functions endowed with the max-norm, is continuously embedded in $L^{\infty}(F)$ because $F$ is compact. By Proposition 2.1.3, we have $C^{0}(F) \subseteq L^{p}(F)$ continuously for all $1 \leq p \leq \infty$ (we shall not need the case $0<p<1$ ). Therefore, all spaces $C^{m}(F)$ of $m$-times real continuously differentiable functions on $F$ are linear subspaces of $L^{p}(F)$.

We define the Sobolev norm to be the functional

$$
\|f\|_{m, p}:=\sum_{|\alpha| \leq m}\left\|\partial^{\alpha} f\right\|_{p},
$$

where $m \in \mathbb{Z}_{>0}, 1 \leq p \leq \infty, \alpha$ is a $d$-dimensional non-negative multi-index with 1 -norm, and $\partial^{\alpha}$ denotes the $\alpha$-th partial derivative. With the above considerations, it is clear that, for all $1 \leq p \leq \infty$, the Sobolev norm is finite on and makes normed spaces of the spaces $C^{m}(F)$. Furthermore, the Sobolev norm dominates the $L^{p}$-norm for the respective $p$. The Sobolev spaces can now be defined naturally: the Sobolev space $W^{m, p}(F)$ for $m \in \mathbb{Z}_{\geq 0}$ and $1 \leq p \leq \infty$ is the closure of $C^{m}(F) \subseteq L^{p}(F)$ with respect to the Sobolev norm $\|\cdot\|_{m, p}$. Clearly, $W^{m, p}(F)$ is a Banach space.

Of course, if $F$ were not compact, such a simple definition of Sobolev spaces would not be possible. Indeed, in order to treat the case of domain $\mathbb{R}^{d}$, Sobolev introduced a weak extension method [Sob63, §6]. Given a multi-index $\alpha \in \mathbb{Z}_{>0}^{d}$, the definition of derivative is extended so that $\partial^{\alpha} f$ is the unique distribution fulfilling

$$
\int\left(f \partial^{\alpha} g-(-1)^{|\alpha|} g \partial^{\alpha} f\right)=0
$$

for all $C^{\infty}$ test functions $g$. The Sobolev space with parameters $(m, p)$ is then given by those $m$-times weakly differentiable distributions for which the Sobolev norm $\|\cdot\|_{m, p}$ is finite.

It is a valid question whether the strong and weak extension method both lead to the same definition of Sobolev space. Since, locally, weak and strong extension are equivalent [Fri44], we have equivalence of definitions with respect to the compact measure space $F$, provided that the closure of $C^{m}(F)$ is taken in an absolutely continuous $L^{p}$-space (see section 2.8 for more information on absolutely continuous spaces). For $1 \leq p<\infty$ the $L^{p^{p}}$ spaces are absolutely continuous (as long as they are defined over a $\sigma$-finite measure space), so for those $p$ our spaces $W^{m, p}(F)$ satisfy the standard definition. In fact, this equivalence can be extended to arbitrary domains [MS64, but again only for $1 \leq p<\infty$. Indeed, for $p=\infty$ equivalence fails. As a counterexample, consider any continuous function which is not strongly differentiable in only one point. As a single point has measure zero, such a function is weakly differentiable, but though the graph of its weak derivative can be exhausted by a sequence of graphs of continuous functions, approximation fails in $L^{\infty}$. Henceforward, we shall consider the Sobolev space $W^{m, p}(F)$ only for $1 \leq p<\infty$.

One of the benefits of Sobolev spaces with respect to differential equations is obvious by now. Sobolev spaces enable us to pose problems involving differential 
operators in the context of Banach spaces. For example, if $f \in L^{p}(F), 1 \leq p<$ $\infty$, the equation

$$
\Delta \psi=f
$$

is an equation in the Banach space $L^{p}(F)$ if we stipulate that $\psi \in W^{2, p}(F)$. In other words, we can seek for solutions $\psi$ in the Banach space $W^{2, p}(F)$.

As such it is worthwhile to investigate the interrelations among the Sobolev spaces $W^{m, p}(F)$ (and hence Lebesgue spaces, as, clearly, $W^{0, p}(F)=L^{p}(F)$ for $1 \leq p<\infty)$. Two such interrelations are immediately obvious. For fixed $m$, an analogon to Proposition 2.1.3 holds, and for fixed $p$, Sobolev spaces defined by higher order derivatives are contained in all lower order spaces. Note that, despite the Sobolev spaces becoming smaller with increasing $m$, they are all dense in their respective $L^{p}$-space, as already the step functions are dense in these spaces [Rie10, $\S 4$ and $\S 16]$ and step functions are clearly weakly differentiable. This can also be seen from the strong viewpoint using approximation through mollifiers [Fri44] (but see also [Sob63, §8] for a similar technique).

A less obvious observation is the fact that requiring a function $f$ to belong to some Sobolev space makes it more regular with respect to $L^{p}$-spaces (or $C^{0}$, in place of $L^{\infty}$ ) with increasing $m$. This is a loose description of a set of results which have collectively become known as Sobolev's theorem. Recall that we have defined $d$ to be the dimension of the torus $F$. Perhaps the most illustrative result as to what degree regularisation occurs is the following

Theorem 2.2.2 (Sobolev Sob63, §10]). Let $f \in W^{d, 1}(F)$, then $f$ is continuous. Moreover, the embedding $W^{d, 1}(F) \subseteq C^{0}(F)$ is bounded.

In other words, for an integrable function to be continuous, it is sufficient for its weak derivatives up to order $\operatorname{dim}(F)$ to be integrable as well. If one doesn't want to go all the way from $p=1$ to $C^{0}$ in one step, the following result is useful.

Theorem 2.2.3 (Sobolev Sob63, $\S 7$ and $\S 10]$ ). Let $l \in \mathbb{Z}_{>0}, m \in \mathbb{Z}_{>0}$ and $1 \leq$ $p<q<\infty$ with $m p<d$ and $q^{-1}=p^{-1}-m d^{-1}$. Then $W^{l+m, p}(F) \subseteq W^{l, q}(F)$ and the embedding is continuous.

Sobolev proved this result only for $l=0$ but since $q<\infty$, the generalisation is obvious.

The embeddings in the last two theorems are optimal in the sense that $C^{0}(F)$ cannot be replaced with $C^{m}(F)$ where $m>0$, or even with the Hölder space $C^{0, \alpha}(F)$ where $\alpha>0$, and $W^{l, q}(F)$ cannot be replaced with a better Sobolev space. Of the optimal embeddings, we need only the two presented, but there are others. In particular, we are working only with the torus $F$, so we can totally avoid the question of what geometric regularity conditions are necessary on the measure space used for certain Sobolev embeddings to exist.

For each optimal embedding, there is a whole family of non-optimal ones. In these cases, the Sobolev embedding may become compact instead of merely continuous. We use this opportunity to insert a digression on compact operators and the important role they play with respect to differential equations. See Yos95] for a general textbook on functional analysis, including the theory of compact operators.

Recall that a compact operator is a linear operator which maps the unit ball into a relatively compact set. In infinite-dimensional vector spaces, the unit 
ball is not compact, so compactness is quite a strong condition. Luckily, the following properties make compact operators easy to identify:

Proposition 2.2.4. (a) In products of compact operators, the following viral behaviour is expressed. Let $E_{1}, E_{2}, E_{3}$ be Banach spaces and let $A_{1}: E_{1} \rightarrow$ $E_{2}$ and $A_{2}: E_{2} \rightarrow E_{3}$ be bounded linear operators. Then the product $A_{2} A_{1}$ is compact if $A_{1}$ or $A_{2}$ is compact.

(b) Let $E_{1}, E_{2}$ be Banach spaces and denote by $\mathcal{L}\left(E_{1}, E_{2}\right)$ the Banach space of bounded linear operators $E_{1} \rightarrow E_{2}$, and by $\mathcal{K}\left(E_{1}, E_{2}\right)$ the subset of compact operators. Then $\mathcal{K}\left(E_{1}, E_{2}\right)$ is a closed subspace of $\mathcal{L}\left(E_{1}, E_{2}\right)$. If $E:=E_{1}=E_{2}$, then $\mathcal{K}(E, E)$ is also a two-sided ideal in $\mathcal{L}(E, E)$.

(c) Let $E$ be a Banach space and $P: E \rightarrow E$ a bounded linear operator of finite rank. Then $P$ is compact. If $E$ has a Schauder basis, then the bounded linear operators of finite rank are dense in $\mathcal{K}(E, E){ }^{6}$

We also state the following slight alteration of a lemma due to F. Riesz.

Proposition 2.2.5. Let $E$ be a Banach space and let $P: E \rightarrow E$ be a compact projector. Then $P$ has finite rank.

Proof. Let $E_{1}$ be the image of $P$ and let $x \in E_{1}$ with $\|x\| \leq 1$, then $x$ lies in the closed unit ball $B_{E}$ of $E$, so, due to $P x=x$, the image $P B_{E}$ contains the closed unit ball $B_{E_{1}}$ of $E_{1}$. Clearly, the space $E_{1}$ is generated by this unit ball. Now, $P B_{E}$ is compact because $P$ is compact. Therefore, the closed set $B_{E_{1}}$ is compact as well, and thus sequentially compact. It now follows from [Rie16, $\S 1$, Hilfssatz 5] that $E_{1}$ is finite-dimensional. Hence $P$ has finite rank.

In dealing with differential equations, one often needs to consider the spectral theory of certain operators. Let $E$ be some Banach space, $D \subseteq E$ a dense subspace and $A: D \rightarrow E$ linear, not necessarily bounded operator whose graph is closed in $E \times E$ (that is, $A$ is a closed operator). Typically, $A$ will be some kind of differential operator. The eigenvalue equation

$$
\lambda x-A x=0,
$$

where the eigenvector $x \in E, x \neq 0$, and the eigenvalue $\lambda \in \mathbb{C}$, is then welldefined in the sense that if, for some sequence $\left(x_{n}\right)_{n \in \mathbb{N}}$ in $D$ converging to $x$, the sequence $\left(A x_{n}\right)_{n \in \mathbb{N}}$ actually does converge to $\lambda x$, then $\lambda x \in D$ due to closedness. Hence, $A$ gives rise to a family of operators $\lambda-A$ parameterised by $\lambda \in \mathbb{C}$. Naturally, we are interested in the set of eigenvalues of $A$. Being in an analytical setting, it seems apt to describe the eigenvalues as the singularities of the mapping

$$
\lambda \mapsto(\lambda-A)^{-1} .
$$

This mapping is called the resolvent of $A$. To make this work, we need a welldefined domain for this mapping which induces sufficiently benign analytical properties. Our domain of choice is the resolvent set

$\rho(A):=\{\lambda \in \mathbb{C}: \lambda-A$ has a bounded inverse whose domain is dense in $E\}$.

\footnotetext{
${ }^{6}$ See Enf73 for a Banach space (without a Schauder basis) providing a counterexample. On the other hand, for most common spaces, especially on $\Gamma^{*}$, it is often not too difficult to construct an explicit Schauder basis.
} 
The remaining $\lambda$ are defined to constitute the spectrum of $A$,

$$
\operatorname{Spec}(A):=\mathbb{C} \backslash \rho(A) .
$$

The resolvent is a nice mapping now, but there are several problems with the spectrum. The definition of eigenvalues through equation (2.2.6) is purely algebraic. In particular, $\lambda \in \mathbb{C}$ is an eigenvalue of $A$ only if $\lambda-A$ does not have an inverse at all. Such elements of $\operatorname{Spec}(A)$ constitute the point spectrum $\operatorname{Spec}_{P}(A)$. Another reason why $\lambda$ might be an element of $\operatorname{Spec}(A)$ is that, while an inverse $(\lambda-A)^{-1}$ exists, its domain is not dense in $E$. Such $\lambda$ constitute the residual spectrum $\operatorname{Spec}_{R}(A)$. Finally, even if an inverse with dense domain exists, it need not be bounded, giving rise to the continuous spectrum $\operatorname{Spec}_{C}(A)$. We have thus arrived at a partition of $\operatorname{Spec}(A) \operatorname{into} \operatorname{Spec}_{P}(A), \operatorname{Spec}_{R}(A)$ and $\operatorname{Spec}_{C}(A)$.

Since there are three types of spectra, in order to make use of the resolvent method, we need to know somehow, what $\lambda \in \operatorname{Spec}(A)$ goes into which spectrum. This is doable for simple operator: 7 , but difficult in general. This situation simplifies considerably if we restrict ourselves to operators $A$ which have a compact power, that is, operators from the radical ideal $\sqrt{\mathcal{K}}(E, E)$ of $\mathcal{K}(E, E)$. The spectral theory of such operators was started by F. Riesz [Rie16, §2] and later completed by Schauder [Sch30], who provided necessary additions in duality theory.

Theorem 2.2.7 (Riesz-Schauder). If $A \in \sqrt{\mathcal{K}}(E, E)$, then each nonzero eigenvalue of $A$ has finite multiplicity. Conversely, each nonzero $\lambda \in \operatorname{Spec}(A)$ is an eigenvalue of $A$ (i.e. $\operatorname{Spec}(A) \backslash\{0\} \subseteq \operatorname{Spec}_{P}(A)$ ). The spectrum of $A$ does not have any accumulation point, with the possible exception of zero.

It turns out that we shall need this theorem for compact operators only, that is, work with $\mathcal{K}(E, E)$ instead of $\sqrt{\mathcal{K}}(E, E)$. This theorem can be reversed in the following sense:

Theorem 2.2.8 (Cauchy-Nagumo Nag36). If $A$ is closed and $\lambda_{0} \in \operatorname{Spec}(A)$ such that $\lambda_{0}$ is not an accumulation point of $\operatorname{Spec}(A)$, then the resolvent of $A$ admits a Laurent expansion

$$
(\lambda-A)^{-1}=\sum_{k=-\infty}^{\infty}\left(\lambda-\lambda_{0}\right)^{k} A_{k}
$$

in a small neighbourhood of $\lambda_{0}$, where

$$
A_{k}=\frac{1}{2 \pi i} \oint_{\lambda_{0}} \frac{(\lambda-A)^{-1}}{\left(\lambda-\lambda_{0}\right)^{k+1}} \mathrm{~d} \lambda .
$$

The residue $A_{-1}$ is a projector. If $\lambda_{0}$ is a pole of the resolvent map, that is, $\left(\lambda-\lambda_{0}\right)^{n}(\lambda-A)^{-1}$ has a removable singularity at $\lambda_{0}$ for sufficiently large $n \in \mathbb{N}$, then $\lambda_{0}$ is an eigenvalue of $A$ and the image of $A_{-1}$ is an $A$-invariant subspace of $E$ equal to the kernel of $\left(\lambda_{0}-A\right)^{n}$.

\footnotetext{
${ }^{7}$ We do not specify the meaning of "simple" in this general setting. Later we shall use the Laplacian and operators related to the nabla operator as simple operators in the sense of this section.
} 
On a technical level, this theorem requires a generalisation of infinitesimal calculus to operator-valued functions allowing for an extension of the Cauchy integral in particular. The contour integral (2.2.9) is meant to be along a small, circular contour anticlockwise around the isolated singularity $\lambda_{0}$ such that no other singularity lies within the contour. Nagumo used a Riemann based integral calculus for this purpose, which we won't analyse in detail. The result will hold for any sufficiently sane integral calculus. Note that, in a different context, we shall introduce the Lebesgue based Bochner integral in section 2.6 .

How do we use Riesz-Schauder theory on differential operators? After all, differential operators are not normally compact, let alone bounded on Lebesgue spaces. The solution is to use Riesz-Schauder theory on a compact resolvent of some simple differential operator to build the resolvent of a more complex operator. So all we have to ensure is that the initial resolvent is compact. One way to achieve that goal is to use non-optimal Sobolev embeddings. The following theorem was proven by Kondrashov [Kon45] building on work by Rellich [Rel30], who had proven the compactness of a certain sublinear integral operator.

Theorem 2.2.10 (Rellich-Kondrashov). Let $l \in \mathbb{Z}_{\geq 0}, m \in \mathbb{Z}_{>0}$ and $1 \leq p<$ $q<\infty$ with $m p \leq d$ and $q^{-1}>p^{-1}-m d^{-1}$. Then $W^{l+m, p}(F) \subseteq W^{l, q}(F)$ and the embedding is compact.

Typically, we will have some simple differential operator $A$ (such as the nabla or the Laplace operator) which maps some Sobolev space boundedly into some Lebesgue space. In order to apply the Rellich-Kondrachov theorem, we would like to have some resolvent $(\lambda-A)^{-1}$ which maps the Lebesgue space into a Sobolev space (which could then be embedded back into a Lebesgue space). As it will turn out, this is not always possible, and this impossibility is the main reason why we need more intricate function spaces.

Let us investigate the target space of $(\lambda-A)^{-1}$. We shall do so in terms of the Laplace operator $\Delta$, which has the following beneficial property immediately derived from Proposition 2.1.6.

Lemma 2.2.11. The application of the Laplace operator on a function defined on $F$ transforms to a multiplication with the non-positive sequence $\left(-4 \pi \kappa^{2}\right)_{\kappa \in \Gamma^{*}}$ under the Fourier operator.

We can now get a handle on the resolvent by considering the operator

$$
(\mathrm{id}-\Delta)^{-m / 2},
$$

which already looks very similar to a resolvent of a simple differential operator. The following theorem gives a precise meaning to this operator.

Theorem 2.2.13. Let $m \in \mathbb{Z}_{>0}$. Then there is a Bessel kernel of $m$-th order $G_{m} \in L^{1}(F)$ such that

$$
\widehat{G}_{m}(\kappa)=\left(1+4 \pi^{2} \kappa^{2}\right)^{-m / 2} .
$$

Proof. Aronszajn and Smith introduced Bessel kernels $G_{m}^{\mathbb{R}^{d}} \in L^{1}\left(\mathbb{R}^{d}\right)$ with the specified Fourier transform AS61. One of the advantages of these kernels is their exponential decay behaviour near infinity. It enables us to define the 
corresponding $\Gamma$-periodic kernels $G_{m} \in L^{1}(F)$ via the absolutely convergent series (cf. GJ87, 7.3])

$$
G_{m}(x):=\sum_{\gamma \in \Gamma} G_{m}^{\mathbb{R}^{d}}(x+\gamma)
$$

We have then

$$
\widehat{G}_{m}(\kappa)=\int_{F} \sum_{\gamma \in \Gamma} G_{m}^{\mathbb{R}^{d}}(x+\gamma) \psi_{-\kappa}(x) \mathrm{d} x=\int_{\mathbb{R}^{d}} G_{m}^{\mathbb{R}^{d}}(x) \psi_{-\kappa}(x) \mathrm{d} x=\widehat{G}_{m}^{\mathbb{R}^{d}}(\kappa)
$$

because $\Gamma$ and $\Gamma^{*}$ are dual.

By this theorem, the operator 2.2 .12 can be defined by the convolution with the Bessel kernel $G_{m}$. Since $G_{m} \in L^{1}(F)$, we can do so in a Lebesgue space setting. Thus, we can define our desired target spaces by

$$
B^{m, p}(F):=\left\{f \in L^{p}(F): \text { There is a } g \in L^{p}(F) \text { such that } f=G_{m} * g\right\},
$$

where $m \in \mathbb{Z}_{>0}$ and $1 \leq p \leq \infty$. It can be shown that the function $g$ is unique, so this definition induces the well-defined norm

$$
\|f\|_{B^{m, p}}:=\|g\|_{p}
$$

making Banach spaces of $B^{m, p}(F)$. The relationship of the Bessel potential spaces $B^{m, p}(F)$ with the Sobolev spaces $W^{m, p}(F)$ for $1 \leq p<\infty$ is as follows.

Theorem 2.2.14 ([Cal61]). Let $m \in \mathbb{Z}_{>0}$.

1. Equivalence of Bessel And Sobolev Spaces. Let either

(a) $1<p<\infty$, or

(b) $m$ be even and $p=d=1$.

Then $B^{m, p}(F)=W^{m, p}(F)$ and the norms of both spaces are equivalent.

2. FAilure of EQuivalence. Let $m$ be even, $p=1$ and $d>1$, then $W^{m, p}(F) \subsetneq B^{m, p}(F)$ and the embedding is continuous, but the norms of the two spaces are not equivalent. If $m$ is odd and $p=1$, there is no inclusion in either direction in any dimension $d$.

In the next few sections, we shall look for ways to circumvent the $p=1$ failure of equivalence.

\subsection{Rearrangement-invariant function spaces}

Rearrangement-invariant function spaces are spaces which are stable under certain measure-preserving transformations. We shall consider such spaces over both $X=F$ and $X=\Gamma^{*}$. In the last two sections we have seen that Lebesgue spaces are not always adequate. We can fit partial differential equations into Banach space theory through Bessel spaces, but they are only useful to us if they can be embedded into some Lebesgue space. Therefore, we shall investigate general function spaces with properties that make them "like" Lebesgue 
spaces. A general reference is BS88, though we shall attempt to cite original contributions where it seems appropriate.

Our mode of creation of such function spaces will invariably be through the norm functional. That is, following the work of Luxemburg [Lux55, we consider functionals $\|\cdot\|: \mathfrak{M}(X) \rightarrow[0, \infty]$ and define function spaces $E$ by

$$
E:=\{f \in \mathfrak{M}(X):\|f\|<\infty\} .
$$

In order to arrive at, essentially, Banach spaces, we impose conditions on $\|\cdot\|$ similar to the conditions (F1) through (F5) from section 2.1. plus some more to ensure Lebesgue likeness:

(B0) Equiabsoluteness: $\|f\|=\||f|\|$ for all $f \in \mathfrak{M}(X)$. In other words, it is sufficient to specify $\|\cdot\|$ on $\mathfrak{M}^{+}(X)$.

(B1) Positivity: $\|f\| \geq 0$ for all $f \in \mathfrak{M}^{+}(X)$ (implied by the range of $\|\cdot\|$ ).

(B2) Regularity: If $f \in \mathfrak{M}^{+}(X)$, then $\|f\|=0$ if and only if $f(x)=0$ for $\mu$-almost all $x \in X$.

(B3) Absolute linearity: $\|\lambda f\|=|\lambda|\|f\|$ for all $\lambda \in \mathbb{C}$ and all $f \in \mathfrak{M}^{+}(X)$.

(B4) Triangle inequality: $\|f+g\| \leq\|f\|+\|g\|$ for all $f, g \in \mathfrak{M}^{+}(X)$.

(B5) The Fatou property: If $f \in \mathfrak{M}^{+}(X)$ and $\left(f_{n}\right)_{n \in \mathbb{N}}$ is a sequence of functions in $\mathfrak{M}^{+}(X)$ such that for $\mu$-almost every $x \in X$, the sequence $\left(f_{n}(x)\right)_{n \in \mathbb{X}}$ is monotonically increasing and converges to $f(x)$, then the sequence of norms $\left(\left\|f_{n}\right\|\right)_{n \in \mathbb{N}}$ is monotonically increasing and converges to $\|f\|$.

(B6) Monotonicity: For all $f, g \in \mathfrak{M}^{+}(X)$, if $f(x) \leq g(x)$ for $\mu$-almost all $x \in X$, then $\|f\| \leq\|g\|$.

(B7) Local finiteness: For all $A \in \Sigma$ with $\mu(A)<\infty,\left\|\chi_{A}\right\|<\infty$. In particular, $E$ contains the constant functions if $X$ is a finite measure space.

(B8) Local integrability: For all $A \in \Sigma$ with $\mu(A)<\infty$ there is a $C>0$ such that

$$
\int_{A} f \mathrm{~d} \mu \leq C\|f\|
$$

for all $f \in \mathfrak{M}^{+}(X)$.

We shall call a space $E$ which arises from a functional $\|\cdot\|$ fulfilling (B0) through (B8) a Banach function space, and its norm a Banach function norm. After taking a quotient like in (2.1.2), Banach function spaces become Banach spaces by virtue of, essentially, (B1) through (B5). Note that the completeness property has been replaced with the stronger Fatou property (see e.g. BS88, Theorem 1.1.6]). The equiabsoluteness property (B0) looks innocuous but implies the important property that $|f| \in E$ if $f \in E$. Hence, (B0) is a simple stability property. The monotonicity property (B6) is implied by the Fatou property (take $f_{n}:=g-(g-f) / n$ ) but important enough to state separately. For example, properties (B1) through (B5) trivially generalise from $\mathfrak{M}^{+}(X)$ to the whole $\mathfrak{M}(X)$ by (B0) with the exception of the triangle inequality (B4), 
which needs (B6) as well. Likewise, monotonicity will enable us to make Banach spaces of Bochner spaces in section 2.6. Note that monotonicity excludes the Sobolev and Bessel potential spaces from the family of Banach function spaces. The local finiteness (B7) property ensures that $E$ contains the compactly supported step functions, allowing for reuse of standard proof techniques for Lebesgue spaces, such as the frequent use of the uniform convergence theorem. Finally, the local integrability condition (B8) ensures that all $f \in E$ are nowhere infinite except on a set of measure zero.

We stated that we wanted our conditions (B0) through (B8) on Banach function spaces in order to generalise the Lebesgue $L^{p}$-spaces. Clearly, $L^{p}(F)$ and $\ell^{p}\left(\Gamma^{*}\right)$ are Banach function spaces for $1 \leq p \leq \infty$. However, for $0<p<1$ this is no longer the case. For one, $p$-integrable functions are not necessarily locally integrable if $p<1$. We can, of course, weaken this property to

(B8') Local $p$-integrability: For all $A \in \Sigma$ with $\mu(A)<\infty$ there is a $C>0$ and a $p>0$ such that

$$
\int_{A} f^{p} \mathrm{~d} \mu \leq C\|f\|
$$

for all $f \in \mathfrak{M}^{+}(F)$.

without losing much. In particular, functions are still finite $\mu$-almost everywhere and convergence in norm still implies convergence in measure. A more serious problem is the failure of the triangle inequality (B4). In fact, the Lebesgue spaces are not locally convex for $0<p<1$, so some things are just not going to work in them as in Banach spaces. On the other hand, all is not lost, as the following lemma shows.

Lemma 2.3.1. Let $f, g \in \mathfrak{M}(X)$. Then for all $0<p<1$,

$$
\|f+g\|_{p}^{p} \leq\|f\|_{p}^{p}+\|g\|_{p}^{p} .
$$

In particular, there is a $C \geq 1$ depending only on $p$ such that

$$
\|f+g\|_{p} \leq C\left(\|f\|_{p}+\|g\|_{p}\right) .
$$

Proof. Since (B6) holds for all Lebesgue spaces, we may assume that $f, g \in$ $\mathfrak{M}^{+}(X)$. On $\mathbb{R}_{0}^{+}$, the mapping $a \mapsto a^{r}$ is monotonically increasing for all $r>0$, and concave for $0<r<1$. Therefore we surely have

$$
\int_{X}(f+g)^{p} \mathrm{~d} \mu \leq \int_{X} f^{p} \mathrm{~d} \mu+\int_{X} g^{p} \mathrm{~d} \mu .
$$

For the remainder of the statement of the lemma, it is therefore sufficient to show

$$
(x+y)^{r} \leq C_{r}\left(x^{r}+y^{r}\right)
$$

for all $x, y \geq 0$ and all $r>0$, where $C_{r} \geq 1$ depends only on $r$. For $0<r \leq 1$ this is implied by concaveness, with $C_{r}=1$. Assuming the claim to be true for an arbitrary $r>0$, we have

$$
\begin{aligned}
(x+y)^{r+1} \leq C_{r}\left(x^{r}+y^{r}\right)(x+y) & \\
& \leq C_{r}\left(x^{r+1}+y^{r+1}+2 \max (x, y)^{r+1}\right) \leq 3 C_{r}\left(x^{r+1}+y^{r+1}\right),
\end{aligned}
$$

proving the claim for all $r>0$ by induction. 
This lemma entices us to put forth the property

(B4') Weak triangle inequality: There is a $p>0$ such that $\|f+g\|^{p} \leq\|f\|^{p}+\|g\|^{p}$ for all $f, g \in \mathfrak{M}^{+}(X)$. In particular, $\|f+g\| \leq C(\|f\|+\|g\|)$, where $C \geq 1$ does not depend on $f$ or $g$.

We call a functional $\|\cdot\|: \mathfrak{M}(X) \rightarrow[0, \infty]$ which fulfils (B0) through (B8), with (B4) replaced with (B4') and (B8) replaced with (B8'), a quasi-norm, and the space $E$ it defines a quasi-Banach function space. Clearly, all Lebesgue spaces are quasi-Banach function spaces. Many properties of and theorems for Banach spaces extend to quasi-Banach spaces. Notable exceptions are theorems requiring the application of the triangle inequality consecutively an arbitrary or even infinite number of times, theorems involving infinite series in particular. One victim is the equivalence of completeness with the Riesz-Fischer property, which is used to show that the Fatou property does in fact imply completeness. Fortunately, it is trivial to augment the standard proof (we use [BS88, Theorem 1.1.6] as a template) of completeness to quasi-Banach function spaces:

Proposition 2.3.2. Let $E$ be a quasi-Banach function space. Then $E$ is complete.

Proof. Let $\left(f_{n}\right)_{n \in \mathbb{N}}$ be a Cauchy sequence. From that sequence, choose a Cauchy subsequence $\left(g_{n}\right)_{n \in \mathbb{N}}$ such that

$$
\sum_{n=1}^{\infty}\left\|g_{n+1}-g_{n}\right\|^{p}<\infty
$$

where $0<p \leq 1$ is chosen to be compatible with (B4'). Now, by the monotonicity property and the weak triangle inequality,

$\left\|\sum_{n=1}^{N}\left(g_{n+1}-g_{n}\right)\right\|^{p} \leq\left\|\sum_{n=1}^{N}\left|g_{n+1}-g_{n}\right|^{p}\right\| \leq \sum_{n=1}^{N}\left\|g_{n+1}-g_{n}\right\|^{p} \leq \sum_{n=1}^{\infty}\left\|g_{n+1}-g_{n}\right\|^{p}$

for all $N \in \mathbb{N}$ and $p \leq 1$. Hence, as in the standard proof,

$$
\sum_{n=1}^{\infty}\left(g_{n+1}(x)-g_{n}(x)\right)
$$

converges pointwise $\mu$-almost everywhere to a function $f$. The remainder follows, as in the standard proof, from [BS88, Lemma 1.1.5 (ii)], which is also valid for quasi-Banach function spaces.

Let us now come to rearrangement-invariant function spaces, a subclass of quasi-Banach function spaces. Let $\sigma: X \rightarrow X$ a mapping such that for all $A \in \Sigma, \sigma^{-1}(A) \in \Sigma$ and

$$
\mu(A)=\mu\left(\sigma^{-1}(A)\right)
$$

so $\sigma$ is a measure-preserving transformation. The Lebesgue spaces have the property that $\|f\|_{p}=\|f \sigma\|_{p}$ for $f \in \mathfrak{M}^{+}(X)$, which is essentially the extension to infinitesimals of the fact that for a finite sequence $\left(a_{n}\right)_{n=1}^{N}$

$$
\sum_{n=1}^{N} a_{n}=\sum_{n=1}^{N} a_{\tau(n)}
$$


for all permutations $\tau$. This property has the advantage that, when we have some question concerning the norm $\|f\|$, we can transform $f$ to a simpler form without altering the norm. In other words, we rearrange $f$. The general idea to rearrange an object to get a simpler form is quite old. In the simple case of continuous functions $f$ in $L^{1}\left([0,1], \mathbb{R}^{+}\right)$it corresponds to altering the graph of $f$ without altering the area between the graph and the $x$-axis, just as if graph and axis were impermeable membranes containing an incompressible fluid (though certainly not all rearrangements allowed in this physical picture are mathematically permissible).

In order to implement this idea with function spaces, we make the following definition. Let $f \in \mathcal{M}(X)$, then we define the distribution function of $f$ by

$$
\begin{aligned}
\delta_{f}:(0, \infty) & \rightarrow[0, \infty], \\
\lambda & \mapsto \mu(\{x \in X:|f(x)|>\lambda\}) .
\end{aligned}
$$

Two measurable functions $f, g$ are called equimeasurable if $\delta_{f}=\delta_{g}$ (note that $f$ and $g$ need not be defined on the same measure space for this definition to make sense) and a quasi-Banach function space $E$ is called rearrangement-invariant if any two equimeasurable $f, g \in E$ have equal norms: $\|f\|=\|g\|$. All Lebesgue spaces are rearrangement-invariant, with $\delta_{f}=\delta_{f \sigma}$ in particular. Moreover, all rearrangement-invariant Banach spaces are restricted by the Lebesgue scale in the following sense:

Theorem 2.3.3. Let $E$ be a rearrangement-invariant Banach space. If $E$ is a Banach space of functions on the torus $F$, then

$$
L^{\infty}(F) \subseteq E \subseteq L^{1}(F) .
$$

If $E$ is a Banach space of sequences on the lattice $\Gamma^{*}$, then

$$
\ell^{1}\left(\Gamma^{*}\right) \subseteq E \subseteq \ell^{\infty}\left(\Gamma^{*}\right) .
$$

In both cases all embeddings are continuous.

These embedding barriers may be broken by going from Banach function spaces to quasi-Banach function spaces.

In section 2.4 we shall introduce a rearrangement-invariant refinement of the Lebesgue spaces. Before we do that, we introduce two further important concepts in the theory of rearrangement-invariant spaces.

First, for $f \in \mathfrak{M}(X)$ define the decreasing rearrangement of $f$ by

$$
\begin{aligned}
f^{\star}:[0, \infty) & \rightarrow[0, \infty] \\
t & \mapsto \inf \left\{\lambda \in(0, \infty): \delta_{f}(\lambda) \leq t\right\} .
\end{aligned}
$$

The following monotonicity property follows immediately from the definitions:

Proposition 2.3.4. Let $f, g \in \mathfrak{M}(X)$ such that $|f(x)| \leq|g(x)|$ for $\mu$-almost all $x \in X$, then

$$
\delta_{f}(t) \leq \delta_{g}(t), \quad f^{\star}(t) \leq g^{\star}(t),
$$

for all $t>0$. 
Loosely speaking, the decreasing rearrangement simply orders the values of $|f|$ in decreasing sequence, in particular $f$ and $f^{\star}$ are equimeasurable. In the case $X=\Gamma^{*}$ it is therefore customary to adopt $\mathbb{Z}_{>0}$ as the domain of $f^{\star}$, i.e. the original definition is discretised and the domain is shifted by one. If $f^{\star}(n)$ tends to zero for $n \rightarrow \infty, f^{\star}$ indeed enumerates all values of $|f|$ with multiplicity, except possibly zero, in decreasing order.

As its name suggests, the decreasing rearrangement is monotonically decreasing. In connexion with this behaviour, the following result can be useful.

Proposition 2.3.5 (Hardy's lemma). Let $g_{1}, g_{2} \in \mathfrak{M}((0, \infty),[0, \infty])$ such that

$$
\int_{0}^{t} g_{1}(x) \mathrm{d} x \leq \int_{0}^{t} g_{2}(x) \mathrm{d} x
$$

holds for all $t>0$. Then

$$
\int_{0}^{\infty} f(x) g_{1}(x) \mathrm{d} x \leq \int_{0}^{\infty} f(x) g_{2}(x) \mathrm{d} x
$$

for all monotonically decreasing functions $f:(0, \infty) \rightarrow[0, \infty]$.

This proposition allows us to estimate more complex expressions from the decreasing rearrangement to define new quasi-norms. In view of a generalisation of Hölder's inequality with respect to such new norms, the following two theorems are of utmost importance.

Theorem 2.3.6 (Hardy-Littlewood inequality [HL30]). Let $f, g \in \mathfrak{M}(X)$, then

$$
\int_{X}|f g| \mathrm{d} \mu \leq \int_{0}^{\mu(X)} f^{\star}(x) g^{\star}(x) \mathrm{d} x .
$$

The next theorem shows that the rearrangement-invariant Banach function norms on $X$ are determined by rearrangement-invariant Banach function norms for functions on $[0, \infty)$.

Theorem 2.3.7 (Luxemburg representation theorem). Let $X$ be a non-atomic $\sigma$-finite measure space (resp. $\left.X=\Gamma^{*}\right)$. Furthermore, let $\|\cdot\|$ be a rearrangementinvariant Banach function norm on $\mathfrak{M}(X)$. Then there is a rearrangementinvariant Banach function norm $\|\cdot\|^{\prime}$ on $\mathfrak{M}\left([0, \infty),[0, \infty]\right.$ ) (or, for $X=\Gamma^{*}$, on $\left.\mathfrak{M}\left(\mathbb{Z}_{>0},[0, \infty]\right)\right)$ such that

for all $f \in \mathfrak{M}(X)$.

$$
\|f\|=\left\|f^{\star}\right\|^{\prime}
$$

We have the following simple corollary to these two theorems:

Corollary 2.3.8. Let $f, g \in \mathfrak{M}(X)$ with the property that

$$
\lim _{t \rightarrow \infty} f^{\star}(t)=\lim _{t \rightarrow \infty} g^{\star}(t)=0,
$$

then

$$
\int_{0}^{t}(f g)^{\star}(s) \mathrm{d} s \leq \int_{0}^{t} f^{\star}(s) g^{\star}(s) \mathrm{d} s
$$

for all $t>0$ (all $t \in \mathbb{Z}_{>0}$ if $X$ is discrete). 
Proof. Consider the case $X=F$ first. Let $t>0$. If $t>\mu(F)$, the claim follows directly from the Hardy-Littlewood inequality (theorem 2.3.6 because the integration over the decreasing rearrangement in the left hand side of 2.3 .10 is a Luxemburg representation of the $L^{1}$-norm in the sense of theorem 2.3.7. Otherwise, there is a measurable set $M \subseteq F$ with $\mu(M)=t$ such that

$$
\int_{M}|f g| \mathrm{d} \mu=\int_{0}^{t}(f g)^{\star}(s) \mathrm{d} s
$$

by [BR80, Lemma 2.2.5]. Now, the Hardy-Littlewood inequality applied on the measure space $M$ yields

$$
\int_{0}^{t}(f g)^{\star}(s) \mathrm{d} s \leq \int_{0}^{\mu(M)}\left(\left.f\right|_{M}\right)^{\star}(s)\left(\left.g\right|_{M}\right)^{\star}(s) \mathrm{d} s=\int_{0}^{t}\left(\left.f\right|_{M}\right)^{\star}(s)\left(\left.g\right|_{M}\right)^{\star}(s) \mathrm{d} s .
$$

Now let $\tilde{f}$ and $\widetilde{g}$ be the extensions of $\left.f\right|_{M}$ and $\left.g\right|_{M}$ to $F$, respectively, obtained by setting them zero off $M$. Then the distribution functions of $\tilde{f}$ and $\left.f\right|_{M}$ as well as of $\widetilde{g}$ and $\left.g\right|_{M}$ are equal. Now clearly, $|\widetilde{f}(x)| \leq|f(x)|$ and $|\widetilde{g}(x)| \leq|g(x)|$ for all $x \in F$. Hence, proposition 2.3.4 implies

$$
\int_{0}^{t}\left(\left.f\right|_{M}\right)^{\star}(s)\left(\left.g\right|_{M}\right)^{\star}(s) \mathrm{d} s \leq \int_{0}^{t} f^{\star}(s) g^{\star}(s) \mathrm{d} s
$$

proving the corollary for $X=F$.

Now, assume $X=\Gamma^{*}$. In this setting, the inequality 2.3.10 becomes, in accordance with our conventions for the decreasing rearrangement,

$$
\sum_{n=1}^{N}(f g)^{\star}(n) \leq \sum_{n=1}^{N} f^{\star}(n) g^{\star}(n)
$$

for all $N \in \mathbb{Z}_{>0}$. Now, due to the property 2.3 .9 and since $\Gamma^{*}$ is discrete, for each $N \in \mathbb{Z}_{>0}$ there is a set $M \subseteq \Gamma^{*}$ with $N$ elements such that

$$
\sum_{\kappa \in M}|f(\kappa) g(\kappa)|=\sum_{n=1}^{N}(f g)^{\star}(n) .
$$

The proof can now proceed as in the case $X=F$.

Finally, let us introduce the Hardy-Littlewood maximal function. For $f \in$ $\mathfrak{M}(X)$, define the maximal function by

$$
\begin{aligned}
f^{\star \star}:(0, \infty) & \rightarrow[0, \infty] \\
t & \mapsto \frac{1}{t} \int_{0}^{t} f^{\star}(x) \mathrm{d} x .
\end{aligned}
$$

The maximal function can be estimated against the decreasing rearrangement and has the advantage of admitting a triangle inequality. 
Proposition 2.3.11. Let $f, g \in \mathfrak{M}(X)$, then $f^{\star}(t) \leq f^{\star \star}(t)$ for all $0<t<\infty$, and

$$
(f+g)^{\star \star}(t) \leq f^{\star \star}(t)+g^{\star \star}(t)
$$

for all $0<t<\infty$.

The maximal function transforms most rearrangement-invariant spaces under our consideration to manifestly the same spaces, with $X$ replaced with a subset of $\mathbb{R}$, in accordance with the Luxemburg representation theorem. There are, however, some notable exceptions, which we shall investigate in the next section.

\subsection{Lorentz-Zygmund spaces}

Lorentz-Zygmund spaces are rearrangement-invariant function spaces which are concrete refinements of the Lebesgue spaces. The scale of Lebesgue spaces has a single parameter, the Lebesgue exponent $p$, with $0<p \leq \infty$, while the scale of Lorentz-Zygmund spaces has two additional parameters, the Lorentz exponent $a$, with $0<a \leq \infty$, and the Zygmund exponent $\alpha$, with $-\infty<\alpha<\infty$. The Lorentz-Zygmund spaces $L^{p, a ; \alpha}(F)$ and $\ell^{p, a ; \alpha}\left(\Gamma^{*}\right)$ are then defined by the following quasi-norms.

Definition 2.4.1 (Lorentz-Zygmund quasi-norms). For $0<p \leq \infty, 0<a \leq$ $\infty$ and $-\infty<\alpha<\infty$ we define the Lorentz-Zygmund quasi-norms $\|\cdot\|_{p, a ; \alpha}$ on $\mathfrak{M}(F)$ by

$$
\|f\|_{p, a ; \alpha}:= \begin{cases}\left(\int_{0}^{\mu(F)}\left(t^{1 / p}\left(1-\log \frac{t}{\mu(F)}\right)^{\alpha} f^{\star}(t)\right)^{a} t^{-1} \mathrm{~d} t\right)^{1 / a}, & \text { if } 0<a<\infty, \\ \sup _{0<t<\mu(F)} t^{1 / p}\left(1-\log \frac{t}{\mu(F)}\right)^{\alpha} f^{\star}(t), & \text { if } a=\infty,\end{cases}
$$

and on $\mathfrak{M}\left(\Gamma^{*}\right)$ by

$$
\|f\|_{p, a ; \alpha}:= \begin{cases}\left(\sum_{n=1}^{\infty}\left(n^{1 / p}(1+\log n)^{\alpha} f^{\star}(n)\right)^{a} n^{-1}\right)^{1 / a}, & \text { if } 0<a<\infty, \\ \sup _{n=1}^{\infty} n^{1 / p}(1+\log n)^{\alpha} f^{\star}(n), & \text { if } a=\infty .\end{cases}
$$

We skip the verification that these definitions actually constitute quasi-norms and direct the reader to BS88 instead. Using Hardy's lemma and the HardyLittlewood inequality, it is easy to see that if $a \neq \infty$ they are norms whenever

$$
\begin{aligned}
t & \mapsto t^{a / p-1}\left(1-\log \frac{t}{\mu(F)}\right)^{a \alpha}, \\
n & \mapsto n^{a / p-1}(1+\log n)^{a \alpha}
\end{aligned}
$$

are non-increasing (there are more cases than these in which the resulting spaces can be made Banach spaces, however).

There are two important special cases of Lorentz-Zygmund spaces. Firstly, if $\alpha=0$, we write $p, a$ instead of $p, a ; 0$ and speak of Lorentz spaces. Secondly, if $p=a$, we write $p ; \alpha$ instead of $p, p ; \alpha$ and speak of Zygmund spaces. Special 
cases of Zygmund spaces were first used in Zyg28, Zyg29, while the Lorentz spaces were invented in Lor50, Lor51. In full generality, Lorentz-Zygmund spaces were first investigated in BR80. The Lebesgue spaces are, of course, also a special case of Lorentz-Zygmund spaces.

Proposition 2.4.2. Let $0<p \leq \infty$. Then $L^{p}(F)=L^{p, p ; 0}(F)$ and $\ell^{p}\left(\Gamma^{*}\right)=$ $\ell^{p, p ; 0}\left(\Gamma^{*}\right)$ and the quasi-norms are equal.

Proof. For $p=a$ and $\alpha=0$, the Lorentz-Zygmund quasi-norms reduce to

$$
\left(\int_{0}^{\mu(F)} f^{\star}(t)^{p} \mathrm{~d} t\right)^{1 / p} \text { and } \sup _{0<t<\mu(F)} f^{\star}(t),
$$

and likewise for $X=\Gamma^{*}$. This proves the claim for $p=\infty$ due to the monotonicity and rearrangement property of $f^{\star}$. For $0<p<\infty, \delta_{f}\left(\lambda^{1 / p}\right)=\delta_{|f|^{p}}(\lambda)$ implies $\left(f^{\star}\right)^{p}=\left(|f|^{p}\right)^{\star}$, finishing the proof due to the equimeasurability of $f$ and $f^{\star}$.

Note that the Lorentz-Zygmund quasi-norm for $1 \leq p \leq \infty, a=p$ and $\alpha=0$ is a Luxemburg representation of the standard $L^{p}$-norm.

We shall now give the Lorentz-Zygmund spaces a similar treatment than the Lebesgue spaces in section 2.1. We begin with the inclusion relations. For $X=\Gamma^{*}$, we need them only in the Lorentz spaces. The conditions for inclusion are relatively simple in this case.

Proposition 2.4.3. Let $0<p, q, a, b \leq \infty$. Then

$$
\ell^{p, a}\left(\Gamma^{*}\right) \subseteq \ell^{q, b}\left(\Gamma^{*}\right)
$$

if one of the following conditions holds:

1. $p<q$,

2. $p=q$ and $a \leq b$.

The inclusions are continuous.

Proof. For $p=q$ and $a \leq b$, this is [BS88, Prop. 4.4.2]. Now, let $p<q$. From the case $p=q$ we already know that $\ell^{p, a}\left(\Gamma^{*}\right) \subseteq \ell^{p, \infty}\left(\Gamma^{*}\right)$. So, for $f \in \ell^{p, a}\left(\Gamma^{*}\right)$ we have

$$
\sup _{n=1}^{\infty} n^{1 / p} f^{\star}(n)<\infty .
$$

Therefore

$$
\sum_{n=1}^{\infty} n^{-c}\left(n^{1 / p} f^{\star}(n)\right)^{d}<\infty
$$

for all $d>0$ and all $c>1$. Choose $d<\min (a, b)$ and set $c=1+d / p-d / q$. Since $p<q, c>1$. This implies

$$
f \in \ell^{q, d}\left(\Gamma^{*}\right) \subseteq \ell^{q, b}\left(\Gamma^{*}\right),
$$

proving the inclusion. Continuity also follows, since the $\ell^{p, \infty}\left(\Gamma^{*}\right) \subseteq \ell^{q, d}\left(\Gamma^{*}\right)$ bound implied by our construction (2.4.4) depends only on $c$ and $d$. 
For $X=F$, the inclusion relations among Lorentz-Zygmund spaces are somewhat more complicated as the following proposition shows.

Proposition 2.4.5 (Sha80, Prop. 3.1]). Let $0<p, q, a, b \leq \infty$ and $-\infty<$ $\alpha, \beta<\infty$. Then

$$
L^{p, a ; \alpha}(F) \subseteq L^{q, b ; \beta}(F)
$$

if and only if at least one of the following conditions holds:

1. $q<p$,

2. $q=p<\infty, a \leq b$ and $\beta \leq \alpha$,

3. $q=p<\infty, a>b$ and $\beta+b^{-1}<\alpha+a^{-1}$,

4. $p=\infty, \alpha+a^{-1} \geq 0$, and either $\alpha \neq 0$ or $a<\infty$ or both (in this case, $\left.L^{p, a ; \alpha}(F)=\{0\}\right)$,

5. $q=p=\infty, \beta+b^{-1}<\alpha+a^{-1}$, and either $\beta+b^{-1}<0$ or $\beta=b^{-1}=0$.

6. $q=p=\infty, a \leq b, \beta+b^{-1}=\alpha+a^{-1}$, and either $\beta+b^{-1}<0$ or $\beta=b^{-1}=0$.

The inclusions are continuous.

Next, we generalise Hölder's inequality to some of the Lorentz-Zygmund spaces.

Theorem 2.4.6 (Hölder's inequality for Lorentz-Zygmund spaces). Let $0<$ $p, q, r, a, b, c \leq \infty$ and $-\infty<\alpha, \beta, \gamma<\infty$ such that

$$
\begin{aligned}
p^{-1}+q^{-1} & =r^{-1}, \\
a^{-1}+b^{-1} & =c^{-1}, \\
\alpha+\beta & =\gamma .
\end{aligned}
$$

Let $f, g \in \mathfrak{M}(X)$. Then the inequality

$$
\|f g\|_{r, c ; \gamma} \leq\|f\|_{p, a ; \alpha}\|g\|_{q, b ; \beta}
$$

holds whenever both sides make sense, provided that one of the following conditions is fulfilled:

$$
\begin{aligned}
& \text { 1. } c<r, \\
& \text { 2. } c=r, \gamma \geq 0 \text { and } X=F, \\
& \text { 3. } c=r, \gamma \leq 0 \text { and } X=\Gamma^{*} \text {. }
\end{aligned}
$$

Proof. If $c=\infty$, then necessarily $a=b=r=p=q=\infty$. Due to monotonicity we have for $X=\Gamma^{*}$

$$
\begin{aligned}
\sup _{n=1}^{\infty}(1+\log n)^{\gamma}(f g)^{\star}(n) & =(f g)^{\star}(1) \leq f^{\star}(1) g^{\star}(1) \\
& =(1+\log 1)^{\alpha} f^{\star}(1)(1+\log 1)^{\beta} g^{\star}(1) \\
& \leq\left(\sup _{n=1}^{\infty}(1+\log n)^{\alpha} f^{\star}(n)\right)\left(\sup _{n=1}^{\infty}(1+\log n)^{\beta} g^{\star}(n)\right) .
\end{aligned}
$$


For $X=F$, only the case $\gamma=0$ is relevant as otherwise the supremum is either zero or infinite. But for $\gamma=0$ we get a special case of the classical Hölder inequality 2.1.1

Now, let $c<\infty$. Under the stated prerequisites, the functions

$$
\begin{aligned}
t & \mapsto t^{c / r-1}\left(1-\log \frac{t}{\mu(F)}\right)^{\gamma c}, \\
n & \mapsto n^{c / r-1}(1+\log n)^{\gamma c}
\end{aligned}
$$

are monotonically decreasing. Hence, we can apply Hardy's lemma 2.3.5 and the Hardy-Littlewood inequality 2.3.8, to wit (for $X=F)$ :

$$
\begin{aligned}
\|f g\|_{r, c ; \gamma}^{c}= & \int_{0}^{\mu(F)} t^{c / r-1}\left(1-\log \frac{t}{\mu(F)}\right)^{\gamma c}(f g)^{\star}(t)^{c} \mathrm{~d} t \\
\leq & \int_{0}^{\mu(F)} t^{c / r-1}\left(1-\log \frac{t}{\mu(F)}\right)^{\gamma c}\left(f^{\star}(t) g^{\star}(t)\right)^{c} \mathrm{~d} t \\
= & \int_{0}^{\mu(F)} t^{c / p} t^{-c / a}\left(1-\log \frac{t}{\mu(F)}\right)^{\alpha c} f^{\star}(t)^{c} \\
& \quad \times t^{c / q} t^{-c / b}\left(1-\log \frac{t}{\mu(F)}\right)^{\beta c} g^{\star}(t)^{c} \mathrm{~d} t
\end{aligned}
$$

Using the classical Hölder inequality:

$$
\begin{aligned}
& \leq\left(\int_{0}^{\mu(F)} t^{a / p-1}\left(1-\log \frac{t}{\mu(F)}\right)^{\alpha a} f^{\star}(t)^{a} \mathrm{~d} t\right)^{c / a} \\
& \quad \times\left(\int_{0}^{\mu(F)} t^{b / q-1}\left(1-\log \frac{t}{\mu(F)}\right)^{\beta b} g^{\star}(t)^{b} \mathrm{~d} t\right)^{c / b} \\
& =\|f\|_{p, a ; \alpha}^{c}\|g\|_{q, b ; \beta}^{c} .
\end{aligned}
$$

For $X=\Gamma^{*}$ the argument runs completely analogously.

We remark that the restrictions on $r$ and $c$ can be removed in most, but not all cases, at the cost of the introduction of a factor (which would depend only on the exponents) in the inequality. One particular case where the restrictions cannot be removed is the case $r=1$ (cf. proposition 2.4.14).

Before we move on to convolution and Fourier transform on Lorentz-Zygmund spaces, we slip in the promised digression on the generalisation of RieszThorin interpolation theory. Let us first outline the class of operators we want to interpolate on.

Definition 2.4.7 (quasi-linear operator). Let $X_{1}, X_{2}$ be $\sigma$-finite measure spaces and let $V \subseteq \mathfrak{M}\left(X_{1}\right)$ be a vector subspace of $\mathfrak{M}\left(X_{1}\right)$. Let $T: V \rightarrow \mathfrak{M}\left(X_{2}\right)$ be an 
operator for which there is a $C \geq 1$, such that for all $f, g \in V$, all $\lambda \in \mathbb{C}$ and $\mu_{2}$-almost all $x \in X_{2}$ the following relations are fulfilled:

$$
\begin{aligned}
|(T(f+g))(x)| & \leq C(|(T f)(x)|+|(T g)(x)|), \\
|(T(\lambda f))(x)| & =|\lambda||(T f)(x)| .
\end{aligned}
$$

Then $T$ is called a quasi-linear operator.

Note that in the cases where $C$ can be chosen equal to one, this definition reduces to the well-known concept of a sub-linear operator, albeit defined on the possibly rather large space $V$. Actual interpolation will be done on subspaces of $V$, so $T$ has to be suitably restricted. Another approach, as originally done by Thorin, would be to define $T$ on dense subsets of all spaces in question. This situation is somewhat reminiscent of the strong and weak extensions with Sobolev spaces. However, our "weak" version of operator definition is more flexible as the conditions on the relationship between operator domains are relatively mild 8 . We make the phrase "suitable restriction" concrete as follows:

Definition 2.4.8. Let $E_{1}, E_{2}$ be quasi-Banach spaces and let $T$ be a quasilinear operator. Assume that $E_{1}$ is contained in the domain of $T$, and that the range of the restriction of $T$ to $E_{1}$ is contained in $E_{2}$. If

$$
\left.T\right|_{E_{1}}: E_{1} \rightarrow E_{2}
$$

is bounded, we call $T$ admissible $E_{1} \rightarrow E_{2}$. To simplify notation, we write $T: E_{1} \rightarrow E_{2}$ to express this admissibility.

In section 2.1, the concept of operator types was introduced. We extend this definition as follows.

Definition 2.4.9 (operator types). Let $T$ be a quasi-linear operator, $X_{1}, X_{2}$ two $\sigma$-finite measure spaces, and $1 \leq p, q \leq \infty$. If

$$
T: L^{p}\left(X_{1}\right) \rightarrow L^{q}\left(X_{2}\right)
$$

then $T$ is said to be of strong type $(p, q)$. Furthermore, if

$$
T: L^{p, 1}\left(X_{1}\right) \rightarrow L^{q, \infty}\left(X_{2}\right),
$$

then $T$ is said to be of weak type $(p, q)$.

While it is technically possible to define operator types for $p, q<1$, it would not fit with the denominations "strong" and "weak" for the restriction to $p, q \geq 1$, together with Propositions 2.4.3 and 2.4.5. implies that a strong type $(p, q)$ operator is also weak type $(p, q)$. At any rate, the interpolation theorems we state are formulated for $1 \leq p, q \leq \infty$ only, although it is possible to extend the theory to $p, q<1$.

We shall now introduce the precise type of operator we wish to do interpolation on. The ideas underlying the following definition are an extension of Thorin's ones due to Calderón Cal66.

\footnotetext{
${ }^{8}$ Specifically, the domains on which $T$ is to be defined must be continuously embeddable into topological subspaces of some common Hausdorff space [BS88 ch. 3]. Since we do interpolation theory only on Lorentz-Zygmund spaces, we can always find a suitable Lebesgue space to fill this role.
} 
Definition 2.4.10. Let $1 \leq p_{1}<p_{2} \leq \infty$ and $1 \leq q_{1}, q_{2} \leq \infty$ with $q_{1}<q_{2}$ or $q_{2}<q_{1}$. Set

$$
m:=\frac{q_{1}^{-1}-q_{2}^{-1}}{p_{1}^{-1}-p_{2}^{-1}} .
$$

This is the slope of the non-horizontal, non-vertical line connecting the pair of points $\sigma:=\left(\left(p_{1}^{-1}, q_{1}^{-1}\right),\left(p_{2}^{-1}, q_{2}^{-1}\right)\right)$, that is, $m \in \mathbb{R} \backslash\{0\}$. Define the Calderón operator $S_{\sigma}$ by

$$
\begin{aligned}
S_{\sigma}: \mathfrak{M}((0, \infty)) & \rightarrow \mathfrak{M}((0, \infty)), \\
\left(S_{\sigma} f\right)(t) & :=t^{-1 / q_{1}} \int_{0}^{t^{m}} s^{1 / p_{1}-1} f(s) \mathrm{d} s+t^{-1 / q_{2}} \int_{t^{m}}^{\infty} s^{1 / p_{2}-1} f(s) \mathrm{d} s .
\end{aligned}
$$

Now let $T$ be a quasi-linear operator such that for all functions $f$ from its domain $\left(S_{\sigma} f^{\star}\right)(1)$ is finite and

$$
(T f)^{\star}(t) \leq C\left(S_{\sigma} f^{\star}\right)(t)
$$

holds for all $0<t<\infty$, where $C>0$ is independent from $f$. Then $T$ is said to be of joint weak type $\left(p_{1}, q_{1} ; p_{2}, q_{2}\right)$.

Beyond the obvious fact that strong operator type implies weak operator type, the following proposition describes further how the strong, weak and joint weak operator types are related to each other.

Proposition 2.4.11 (BS88, Theorem 4.4.11]). Let $1 \leq p_{1}<p_{2} \leq \infty$ and $1 \leq q_{1}, q_{2} \leq \infty$ with $q_{1}<q_{2}$ or $q_{2}<q_{1}$. Let $T$ be a quasi-linear operator. Then

1. If $p_{2}<\infty$, then $T$ is of joint weak type $\left(p_{1}, q_{1} ; p_{2}, q_{2}\right)$ if and only if it is of weak types $\left(p_{1}, q_{1}\right)$ and $\left(p_{2}, q_{2}\right)$.

2. If $p_{2}=\infty$ and $T$ is of joint weak type $\left(p_{1}, q_{1} ; p_{2}, q_{2}\right)$, then it is of weak type $\left(p_{1}, q_{1}\right)$.

3. If $p_{2}=\infty$ and $T$ is of weak type $\left(p_{1}, q_{1}\right)$ and of strong type $\left(p_{2}, q_{2}\right)$, then it is of joint weak type $\left(p_{1}, q_{1} ; p_{2}, q_{2}\right)$.

We can now state the interpolation theorem. It was first proven by Józef Marcinkiewicz Mar39, Zyg56 for Lebesgue spaces and later generalised by Bennett and Rudnick [BR80].

Theorem 2.4.12 (Marcinkiewicz interpolation theorem for Lorentz-Zygmund spaces). Let $X_{1}, X_{2} \in\left\{F, \Gamma^{*}\right\}$ and $T$ a quasi-linear operator which maps functions on $X_{1}$ to function on $X_{2}$. Let $1 \leq p_{1}<p_{2} \leq \infty, 1 \leq q_{1}, q_{2} \leq \infty$ with $q_{1}<q_{2}$ or $q_{2}<q_{1}$ and suppose $T$ is of joint weak type $\left(p_{1}, q_{1} ; p_{2}, q_{2}\right)$. Then the following results hold.

1. Intermediate CASe. Let $0<\theta<1$ and set

$$
p:=\left(\frac{1-\theta}{p_{1}}+\frac{\theta}{p_{2}}\right)^{-1}, \quad q:=\left(\frac{1-\theta}{q_{1}}+\frac{\theta}{q_{2}}\right)^{-1} .
$$

Then for all $0<a \leq \infty$ and all $-\infty<\alpha<\infty$,

$$
T: L^{p, a ; \alpha}\left(X_{1}\right) \rightarrow L^{q, a ; \alpha}\left(X_{2}\right),
$$


with

$$
\|T\| \leq \frac{C}{\theta(1-\theta)},
$$

where $C \geq 0$ does not depend on $p, q$ or $\theta$.

2. LeFt EndPoint CASE. Let $1 \leq a \leq b \leq \alpha^{9}$ and $-\infty<\alpha, \beta<\infty$ with $a^{-1}+\alpha=b^{-1}+\beta$. Set $K:=a^{-1}+\alpha=b^{-1}+\beta$. Then

$T: L^{p_{1}, a ; \alpha+1}(F) \rightarrow L^{q_{1}, b ; \beta}(F) \quad$ if $\left(X_{1}, X_{2}\right)=(F, F), q_{1}<q_{2}$ and $K>0$,

$T: L^{p_{1}, a ; \alpha+1}(F) \rightarrow \ell^{q_{1}, b ; \beta}\left(\Gamma^{*}\right) \quad$ if $\left(X_{1}, X_{2}\right)=\left(F, \Gamma^{*}\right), q_{2}<q_{1}$ and $K>0$,

$T: \ell^{p_{1}, a ; \alpha+1}\left(\Gamma^{*}\right) \rightarrow L^{q_{1}, b ; \beta}(F) \quad$ if $\left(X_{1}, X_{2}\right)=\left(\Gamma^{*}, F\right), q_{2}<q_{1}$ and $K<0$,

$T: \ell^{p_{1}, a ; \alpha+1}\left(\Gamma^{*}\right) \rightarrow \ell^{q_{1}, b ; \beta}\left(\Gamma^{*}\right) \quad$ if $\left(X_{1}, X_{2}\right)=\left(\Gamma^{*}, \Gamma^{*}\right), q_{1}<q_{2}$ and $K<0$.

3. Right endpoint Case. Let $1 \leq a \leq b \leq \infty$ and $-\infty<\alpha, \beta<\infty$ with $a^{-1}+\alpha=b^{-1}+\beta$. Set $K:=a^{-1}+\alpha=b^{-1}+\beta$. Then

$T: L^{p_{2}, a ; \alpha+1}(F) \rightarrow L^{q_{2}, b ; \beta}(F) \quad$ if $\left(X_{1}, X_{2}\right)=(F, F), q_{1}<q_{2}$ and $K<0$,

$T: L^{p_{2}, a ; \alpha+1}(F) \rightarrow \ell^{q_{2}, b ; \beta}\left(\Gamma^{*}\right) \quad$ if $\left(X_{1}, X_{2}\right)=\left(F, \Gamma^{*}\right), q_{2}<q_{1}$ and $K<0$,

$T: \ell^{p_{2}, a ; \alpha+1}\left(\Gamma^{*}\right) \rightarrow L^{q_{2}, b ; \beta}(F) \quad$ if $\left(X_{1}, X_{2}\right)=\left(\Gamma^{*}, F\right), q_{2}<q_{1}$ and $K>0$,

$T: \ell^{p_{2}, a ; \alpha+1}\left(\Gamma^{*}\right) \rightarrow \ell^{q_{2}, b ; \beta}\left(\Gamma^{*}\right) \quad$ if $\left(X_{1}, X_{2}\right)=\left(\Gamma^{*}, \Gamma^{*}\right), q_{1}<q_{2}$ and $K>0$.

Let us collect the joint weak type of some standard operators to see how we can apply the Marcinkiewicz interpolation theorem to them.

Proposition 2.4.13. We have the following operator types:

1. The Fourier operator $\mathcal{F}$ is of joint weak type $(1, \infty ; 2,2)$.

2. Let $f \in L^{p}(X)$ with $1 \leq p \leq \infty$, then the operator

$$
g \mapsto f * g
$$

is of joint weak type $\left(1, p ; p^{\prime}, \infty\right)$,

where $p^{\prime}=p /(p-1)$ is the dual exponent to $p$.

Proof. We have already established that the Fourier operator is both of strong types $(1, \infty)$ and $(2,2)$. That the operator $g \mapsto f * g$ is of strong types $(1, p)$ and $\left(p^{\prime}, \infty\right)$ follows from Young's inequality for $p$-integrable functions. Proposition 2.4.11 now yields the desired result.

Another result directly related to theorem 2.4.12 is the following result for the maximal function (see [HL30, theorem 12]):

Proposition 2.4.14. For $1<p \leq \infty$, the Hardy-Littlewood maximal operator $f \mapsto f^{\star \star}$ maps $L^{p}(F)$ boundedly into $L^{p}\left(\mathbb{R}_{>0}\right)$. If, however, $f \in L^{1}(F)$, then $f^{\star \star} \in L^{1}\left(\mathbb{R}_{>0}\right)$ if and only if $f \in L^{1,1 ; 1}(F)$.

\footnotetext{
${ }^{9}$ In their original statement of the theorem [BR80 Theorem C], Bennett and Rudnick have the additional restriction $a \neq b$. This restriction is unnecessary, as one can see in their proof in section 18; see also their remarks 18.2 and BS88, Theorem 4.6.14].
} 
In conclusion, let us remark two things. Firstly, a more general version of Young's inequality for target spaces $L^{r}$ with $1<r<\infty$ can be found in O'N63, Blo72. However, we shall be more interested in the case $r=\infty$. We have presented interpolation theory only for Lorentz-Zygmund spaces but in fact, more general treatments are possible. In the context of Young's inequality, let us just mention the following result.

Proposition 2.4.15. Let $E$ be any rearrangement-invariant function space over $X$ and let $f \in L^{1}(X)$. Then the operator

$$
g \mapsto f * g
$$

maps $E$ boundedly into itself, and we have

$$
\|f * g\|_{E} \leq\|f\|_{1}\|g\|_{E} .
$$

Proof. Again, for $E=L^{1}(X)$ and $E=L^{\infty}(X)$, this follows from the classical Young's inequality. Given theorem 2.3.3 the claim now follows from BS88, Theorem 3.2.2].

Secondly, the fact that the Fourier operator is actually of strong type $(2,2)$ but the Marcinkiewicz theorem assumes only weak type $(2,2)$ makes the right endpoint case useless in this case. Sharp right endpoint results apart from Parseval's identity, such as [Boc98], appear to be sparse in the literature.

\section{$2.5 \quad$ Fourier spaces}

As we have seen in section 2.1, the Fourier transform is valid on the very general spaces $\mathfrak{S}^{*}(F)$ and $\mathfrak{s}^{*}\left(\Gamma^{*}\right)$. The drawback of these spaces is that they lack the analytical properties of (quasi-)Banach function spaces. In addition, the space $\mathfrak{S}^{*}(F)$ is even algebraically deficient in that it does not have a well-defined multiplication. Therefore, we generally prefer the Lorentz-Zygmund spaces (or, more generally, the rearrangement-invariant spaces) of functions on $F$ or $\Gamma^{*}$, and use the Fourier transform to change between these spaces. This, however, gives rise to new problems. For one, the Fourier transform is "only" of strong types $(1, \infty)$ and $(2,2)$ but not, say, $(\infty, 1)$. A more serious problem are the inherent difficulties associated with building bounded linear operators based on the Fourier transform in Bochner spaces (see section 2.6). We would also like to avoid problems with summability and norm convergence of Fourier series. Therefore, we define a kind of space with a Fourier transform already built in.

Definition 2.5.1 (Fourier space). For $E \subseteq \mathfrak{S}^{*}(F)$ set

$$
\mathcal{F} E:=\left\{f \in \mathfrak{s}^{*}\left(\Gamma^{*}\right): \mathcal{F}^{\prime} f \in E\right\},
$$

and for $E \subseteq \mathfrak{s}^{*}\left(\Gamma^{*}\right)$ set

$$
\mathcal{F} E:=\left\{f \in \mathfrak{S}^{*}(F): \mathcal{F} f \in E\right\} .
$$

If $E$ is a normed space, we call $\mathcal{F} E$ the Fourier space of $E$ and define the Fourier space norm on it by

$$
\|f\|:=\|\mathcal{F} f\|_{E} .
$$

In the special case where $E$ is a Lorentz-Zygmund space with exponents $(p, a ; \alpha)$, we denote the Fourier space norm by $\|\cdot\|_{\mathcal{F} p, a ; \alpha}$. 
Direct calculation readily shows

Proposition 2.5.2. If $E \subseteq \mathfrak{S}^{*}(F)$ or $E \subseteq \mathfrak{s}^{*}\left(\Gamma^{*}\right)$ fulfils one or more of the properties (F1) through (F5) from section 2.1, then so does $\mathcal{F} E$.

In particular, if $E$ is a Banach space, then so is its Fourier space. Note, however, that some of the properties from section 2.3 , especially monotonicity and rearrangement invariance, are generally not preserved.

Arguably, Fourier spaces add a layer of indirection to the concreteness of rearrangement-invariant spaces (Lorentz-Zygmund spaces in particular). Our most important standard operations, however, namely pointwise multiplication and convolution, carry over through the Fourier transform by an appropriate extension of the convolution theorem 2.1.10. Moreover, the following generalisations of Hölder's and Young's inequality hold:

Theorem 2.5.3 (Hölder's inequality for Lorentz-Zygmund-Fourier spaces). Let $1 \leq p_{0} \leq \infty$ and $f \in \mathcal{F} L^{p_{0}}(X)$. Set $p_{0}^{\prime}:=p_{0} /\left(p_{0}-1\right)$ and

$$
\begin{array}{ll}
p_{1}:=1, & q_{1}:=p_{0}, \\
p_{2}:=p_{0}^{\prime}, & q_{2}:=\infty .
\end{array}
$$

Then the multiplication operator

$$
g \mapsto f g
$$

maps $\mathcal{F} L^{p, a ; \alpha}(X)$ boundedly into $\mathcal{F} L^{q, b ; \beta}(X)$, where $p, q, a, b, \alpha, \beta$ are chosen in accordance with the Marcinkiewicz interpolation theorem 2.4.12.

Proof. By the convolution theorem we have

$$
\|f g\|_{\mathcal{F} q, b ; \beta}=C\|\widehat{f} * \widehat{g}\|_{q, b ; \beta}
$$

for some $C>0$. The claim now follows from proposition 2.4 .13

Theorem 2.5.4 (Young's inequality for Lorentz-Zygmund-Fourier spaces). Choose $0<p, q, r, a, b, c \leq \infty,-\infty<\alpha, \beta, \gamma<\infty$ such that they fulfil the three relations of theorem 2.4.6. Let $f \in \mathcal{F} L^{p, a ; \alpha}(X)$ and $g \in \mathcal{F} L^{q, b ; \beta}(X)$. Then

$$
\|f * g\|_{\mathcal{F} r, c ; \gamma} \leq\|f\|_{\mathcal{F} p, a ; \alpha}\|g\|_{\mathcal{F} q, b ; \beta}
$$

provided the convolution is defined ${ }^{10}$ and one of the following conditions holds:

$$
\begin{aligned}
& \text { 1. } c<r \text {, } \\
& \text { 2. } c=r, \gamma \leq 0 \text { and } X=F \text {, } \\
& \text { 3. } c=r, \gamma \geq 0 \text { and } X=\Gamma^{*} \text {. }
\end{aligned}
$$

Proof. Once again, this follows from Theorem 2.4.6 by using the convolution theorem. Note that in the case $c=r$, the inequalities for $\gamma$ reverse due to the change of measure space induced by the Fourier transform.

\footnotetext{
${ }^{10}$ recall that we did not embed all Lorentz-Zygmund spaces on $F$ into $\mathfrak{S}^{*}(F)$
} 


\subsection{Bochner spaces and tensor products}

So far, we have only considered functions with values in the complex numbers (possibly with infinity). In this section we shall investigate functions taking values in a Banach space. In our case, this Banach space will usually be a Banach function space, so that, in essence, we are considering functions of the form

$$
X_{1} \rightarrow\left(X_{2} \rightarrow \mathbb{C}\right) .
$$

By currying ${ }^{11}$ such a function has the equivalent form

$$
X_{1} \times X_{2} \rightarrow \mathbb{C} .
$$

Therefore, the theory of Banach space valued functions can be used to treat multivariate complex-valued functions. However, there is an important restriction. The signature 2.6.2 can be curried again to

$$
X_{2} \rightarrow\left(X_{1} \rightarrow \mathbb{C}\right),
$$

giving (2.6.1) with $X_{1}$ and $X_{2}$ swapped. But once we add an analytical structure on spaces of such functions (see below), these two representations will, in general, be different.

The Bochner integral for Banach space valued functions is defined in a similar fashion as the Lebesgue integral for complex-valued functions. It was introduced in Boc33. Let $(X, \Sigma, \mu)$ be a $\sigma$-finite measure space and $E$ be a complex Banach space. We first define the Bochner integral for step functions.

Definition 2.6.3 (Bochner integral for step functions). Let $f: X \rightarrow E$ be a step function, that is, $f$ has a representation

$$
f(x)=\sum_{k=1}^{n} e_{k} \chi_{M_{k}}(x),
$$

where $M_{1}, \ldots, M_{n} \in \Sigma$ have finite measure, and $e_{1}, \ldots, e_{n} \in E$. Then we define the Bochner integral of $f$ by

$$
\int_{X} f \mathrm{~d} \mu:=\sum_{k=1}^{n} e_{k} \mu\left(M_{k}\right) .
$$

It is easy to see that the Bochner integral is independent of the particular representation (2.6.4). We now introduce Bochner-measurable functions through a limiting process.

Definition 2.6.5 (Bochner-measurable function). A function $f: X \rightarrow E$ is called Bochner-measurable if there is a sequence $\left(f_{n}: X \rightarrow E\right)_{n \in \mathbb{N}}$ of step functions such that

$$
\lim _{n \rightarrow \infty}\left\|f_{n}(x)-f(x)\right\|=0
$$

for $\mu$-almost all $x \in X$. Here, $\|\cdot\|$ is the norm of $E$. We denote the space of Bochner measurable functions $X \rightarrow E$ by $\mathfrak{M}(X, E)$.

\footnotetext{
${ }^{11}$ so named in honour of Haskell Curry
} 
A further limiting process now generalises the Bochner integral to all of the Bochner-measurable functions.

Definition 2.6.6 (Bochner integral). Let $f \in \mathfrak{M}(X, E)$. Then $f$ is said to be Bochner integrable if there is a sequence of step functions $\left(f_{n}\right)_{n \in \mathbb{N}}$ as in definition 2.6.5 such that

$$
\lim _{n \rightarrow \infty} \int_{X}\left\|f_{n}(x)-f(x)\right\| \mathrm{d} \mu=0 .
$$

In this case, the Bochner integral of $f$ is given by

$$
\int_{X} f \mathrm{~d} \mu:=\lim _{n \rightarrow \infty} \int_{X} f_{n} \mathrm{~d} \mu .
$$

This definition makes sense due to the Bochner-measurability of $f$ and the completeness of $E$. The set of Bochner integrable functions is characterised by Bochner's theorem.

Theorem 2.6.7 (Bochner). A function $f \in \mathfrak{M}(X, E)$ is Bochner-integrable if and only if the function

$$
x \mapsto\|f(x)\|
$$

is Lebesgue-integrable. In this case

$$
\left\|\int_{X} f(x) \mathrm{d} x\right\| \leq \int_{X}\|f(x)\| \mathrm{d} x .
$$

Note that in particular, the function 2.6 .8 induces a norm on the Bochnerintegrable functions, making them a Banach space. We denote this space by $L^{1}(X, E)$. This scheme of creating a Banach space of Banach space valued functions can be generalised to all Banach function spaces.

Theorem 2.6.9. Let $E_{1}(X)$ be a Banach function space over the measure space $X$ and let $E_{2}$ be another Banach space (not necessarily a Banach function space). Then the space

$$
E_{1}\left(X, E_{2}\right):=\left\{f \in \mathfrak{M}\left(X, E_{2}\right):\|f(\cdot)\|_{E_{2}} \in E_{1}(X)\right\}
$$

is a Banach space with norm

$$
\|f\|:=\|\| f(\cdot)\left\|_{E_{2}}\right\|_{E_{1}} .
$$

Proof. Properties (F1) and (F3) from section 2.1 are obvious. If $\|f\|=0$, then we have $f(x)=0$ for $\mu$-almost all $x \in X$ since $E_{1}(X)$ and $E_{2}$ are Banach (function) spaces. This shows (F2) (with the usual reservations as in (2.1.2)). To establish the triangle inequality (F4), let $f, g \in E_{1}\left(X, E_{2}\right)$. Then

$$
\|f+g\|=\|\|(f+g)(\cdot)\left\|_{E_{2}}\right\|_{E_{1}}=\|\| f(\cdot)+g(\cdot)\left\|_{E_{2}}\right\|_{E_{1}}
$$

Due to the triangle inequalities in $E_{2}$ and $E_{1}(X)$ and the monotonicity property (B6) of $E_{1}(X)$ :

$$
\leq\|\| f(\cdot)\left\|_{E_{2}}+\right\| g(\cdot)\left\|_{E_{2}}\right\|_{E_{1}} \leq\|f\|+\|g\| .
$$


This also shows that $E_{1}\left(X, E_{2}\right)$ is a vector space. Finally, let $\left(f_{n}\right)_{n \in \mathbb{N}}$ be a sequence in $E_{1}\left(X, E_{2}\right)$ such that

$$
\sum_{n \in \mathbb{N}}\left\|f_{n}\right\|<\infty .
$$

Since $E_{1}(X)$ is complete, there is a $g \in E_{1}(X)$ such that

$$
g=\sum_{n \in \mathbb{N}}\left\|f_{n}(\cdot)\right\|_{E_{2}}, \quad\|g\|_{E_{1}} \leq \sum_{n \in \mathbb{N}}\left\|f_{n}\right\| .
$$

Since $g$ is finite-valued $\mu$-almost everywhere,

$$
\sum_{n \in \mathbb{N}}\left\|f_{n}(x)\right\|_{E_{2}}<\infty
$$

holds for $\mu$-almost all $x \in X$. Since $E_{2}$ is complete, there exists a $\mu$-almost everywhere defined function $f \in \mathfrak{M}\left(X, E_{2}\right)$ such that

$$
f(x)=\sum_{n \in \mathbb{N}} f_{n}(x), \quad\|f(x)\|_{E_{2}} \leq \sum_{n \in \mathbb{N}}\left\|f_{n}(x)\right\|,
$$

for those $x \in X$ for which $f$ is defined. Now $\|f(x)\|_{E_{2}} \leq g(x)$ holds $\mu$-almost everywhere, so $\|f(\cdot)\|_{E_{2}} \in E_{1}$ by the monotonicity property (B6). In particular, $f \in E_{1}\left(X, E_{2}\right)$ and

$$
\|f\| \leq \sum_{n \in \mathbb{N}}\left\|f_{n}\right\| .
$$

Therefore, $E_{1}\left(X, E_{2}\right)$ is complete.

We have not found the above theorem in the literature, despite the simple and standard proof. Less general versions (say, with Lebesgue spaces in place of Banach function spaces) are commonplace, however. Clearly, the same proof can be used for quasi-Banach function spaces $E_{2}$ as well, with the Riesz-Fischer property replaced by a suitable $p$-summed version of the Riesz-Fischer property, resulting in $E_{1}\left(X, E_{2}\right)$ also being a quasi-Banach space.

Let $E_{1}(X)$ and $G_{1}(Y)$ be two Banach function spaces over measure spaces $X$ and $Y$, respectively, and let $E_{2}, G_{2}$ be arbitrary Banach spaces. Given bounded linear operators

$$
A_{1}: E_{1}(X) \rightarrow G_{1}(Y), \quad A_{2}: E_{2} \rightarrow G_{2},
$$

we shall want to define a "combined" linear operator

$$
E_{1}\left(X, E_{2}\right) \rightarrow G_{1}\left(Y, G_{2}\right) .
$$

By the local finiteness property $(\mathrm{B} 7)$, the space $E_{1}\left(X, E_{2}\right)$ surely contains all step functions of the type 2.6.4 . It is therefore natural to stipulate that the combination of $A_{1}$ and $A_{2}$ should operate on a step function $f$ as

$$
f=\sum_{k=1}^{n} \chi_{M_{k}} e_{k} \mapsto \sum_{k=1}^{n} A_{1}\left(\chi_{M_{k}}\right) A_{2}\left(e_{k}\right),
$$

where $M_{1}, \ldots, M_{n} \in \Sigma_{X}$ have finite measure and $e_{1}, \ldots, e_{n} \in E_{2}$. In order to generalise this kind of operation to arbitrary elements of $E_{1}\left(X, E_{2}\right)$, we shall make use of Banach space tensor products. The relevant theory was introduced by Schatten Sch50] and Grothendieck Gro55. 
Definition 2.6.11 (tensor product). Let $V_{1}, V_{2}$ be complex vector spaces and let $\operatorname{Bil}\left(V_{1}, V_{2}\right)$ be the space of bilinear forms on $V_{1} \times V_{2}$. For $x_{1} \in V_{1}$ and $x_{2} \in V_{2}$ we define the tensor $x_{1} \otimes x_{2}$ by

$$
\begin{aligned}
x_{1} \otimes x_{2}: \operatorname{Bil}\left(V_{1}, V_{2}\right) & \rightarrow \mathbb{C}, \\
A & \mapsto A\left(x_{1}, x_{2}\right) .
\end{aligned}
$$

The subspace $V_{1} \otimes V_{2}$ of $\operatorname{Hom}\left(\operatorname{Bil}\left(V_{1}, V_{2}\right), \mathbb{C}\right)$ generated by all tensors $x_{1} \otimes x_{2}$, $x_{1} \in V_{1}, x_{2} \in V_{2}$ is called the tensor product of $V_{1}$ and $V_{2}$.

This concept of a tensor product is purely algebraical. The following proposition provides an analytical construct on the tensor product.

Proposition 2.6.12. Let $E_{1}(X)$ be a Banach function space and $E_{2}$ be a Banach space. Define a linear map

$$
\begin{aligned}
J: E_{1}(X) \otimes E_{2} & \rightarrow E_{1}\left(X, E_{2}\right) \\
\text { by linear extension of } f \otimes e & \mapsto f(\cdot) e .
\end{aligned}
$$

Then $J$ is injective, making a normed space of $E_{1}(X) \otimes E_{2}$ through the norm on $E_{1}\left(X, E_{2}\right)$. Let $E_{1}(X) \widehat{\otimes} E_{2}$ be the completion of $E_{1}(X) \otimes E_{2}$ with respect to this norm, then $J$ extends to an isomorphism from $E_{1}(X) \widehat{\otimes} E_{2}$ to a Banach subspace of $E_{1}\left(X, E_{2}\right)$ containing the step functions.

Proof. Let $f_{1}, f_{2} \in E_{1}(X)$ and $e_{1}, e_{2} \in E_{2}$ and assume $f_{1}(\cdot) e_{1}=f_{2}(\cdot) e_{2}$. If $f_{1}=0$ or $e_{1}=0$ then $f_{2}=0$ or $e_{2}=0$. Otherwise, $\left\{e_{1}, e_{2}\right\}$ is linearly dependent. Without restriction, $e_{1}=e_{2}$ in this case, and thus $f_{1}=f_{2}$. It follows that $f_{1} \otimes e_{1}=f_{2} \otimes e_{2}$ in all cases. The norm of $E_{1}\left(X, E_{2}\right)$ can now be transported back to $E_{1}(X) \otimes E_{2}$, naturally making $J$ continuous. The natural extension of $J$ to the completion then leads to a closed subspace of $E_{1}\left(X, E_{2}\right)$ which contains the step functions because $E_{1}(X)$ contains the step functions by (B7).

Note that the step functions are dense in many Banach function spaces, so we shall have $E_{1}(X) \widehat{\otimes} E_{2} \cong E_{1}\left(X, E_{2}\right)$ very often. In particular, the step functions are dense in $L^{p, a ; \alpha}(X)$ for $1 \leq p \leq \infty, 1 \leq a<\infty$ and $-\infty<\alpha<\infty$ (whenever this space is a Banach function space). If $a=\infty$, the step functions are, in general, not dense. For example, $\ell^{\infty}\left(\Gamma^{*}\right) \widehat{\otimes} E_{2} \subsetneq \ell^{\infty}\left(\Gamma^{*}, E_{2}\right)$ in general. In this particular case, it is often possible to use the subspace $c^{0}\left(\Gamma^{*}\right) \subseteq \ell^{\infty}\left(\Gamma^{*}\right)$ of sequences vanishing at infinity instead, in which the step functions are dense again. If it is not possible to somehow withdraw from $\ell^{\infty}\left(\Gamma^{*}, E_{2}\right)$ to $\ell^{\infty}\left(\Gamma^{*}\right) \widehat{\otimes} E_{2}$, tensor product theory cannot be used and the Bochner spaces have to be used directly.

Anyway, coming back to our bounded linear operators $A_{1}, A_{2}$ from (2.6.10), the prescription

$$
\left(A_{1} \otimes A_{2}\right)\left(e_{1} \otimes e_{2}\right):=\left(A_{1} e_{1}\right) \otimes\left(A_{2} e_{2}\right), \quad e_{1} \in E_{1}(X), e_{2} \in E_{2},
$$

extends to a linear operator $E_{1}\left(X, E_{2}\right) \rightarrow G_{1}\left(Y, G_{2}\right)$ in many cases (at least when the step functions are dense in $\left.E_{1}\left(X, E_{2}\right)\right)$. One problem with this definition is that even though $A_{1}$ and $A_{2}$ are continuous, $A_{1} \otimes A_{2}$ is not always so. We shall now identify the most important cases when $A_{1} \otimes A_{2}$ is continuous. 
Proposition 2.6.13. Let $E(X)$ be a Banach function space, $G_{1}, G_{2}$ be two arbitrary Banach spaces and $A: G_{1} \rightarrow G_{2}$ a bounded linear operator. Then

$$
\operatorname{id} \otimes A: E(X) \widehat{\otimes} G_{1} \rightarrow E(X) \widehat{\otimes} G_{2}
$$

is bounded with $\|\operatorname{id} \otimes A\| \leq\|A\|$.

Proof. It is sufficient to prove boundedness on the dense subspace $E(X) \otimes G_{1}$. Let

$$
\sum_{k=1}^{n} f_{k} \otimes x_{k} \in E(X) \otimes G_{1},
$$

then, using the isometry $J$ :

$$
\begin{aligned}
\left\|(\mathrm{id} \otimes A)\left(\sum_{k=1}^{n} f_{k} \otimes x_{k}\right)\right\| & =\|\| \sum_{k=1}^{n} f_{k}(\cdot) A x_{k}\left\|_{G_{2}}\right\|_{E} \\
& =\|\| A\left(\sum_{k=1}^{n} f_{k}(\cdot) x_{k}\right)\left\|_{G_{2}}\right\|_{E}
\end{aligned}
$$

By monotonicity:

$$
\begin{aligned}
& \leq\|\| A\|\| \sum_{k=1}^{n} f_{k}(\cdot) x_{k}\left\|_{G_{1}}\right\|_{E} \\
& =\|A\|\left\|\sum_{k=1}^{n} f_{k} \otimes x_{k}\right\| .
\end{aligned}
$$

For continuity to be inherited by the left factor, we need an additional property on the linear operator. We call a linear operator $A$ on a Banach function space positive, if

$$
\left|\left(A \chi_{M}\right)(x)\right|=\left(A \chi_{M}\right)(x)
$$

for all $M \in \Sigma$ of finite measure and $\mu$-almost all $x$.

Proposition 2.6.14. Let $E_{1}(X)$ be a Banach function space in which the step functions are dense. Let $E_{2}(Y)$ be another Banach function space and let $A: E_{1}(X) \rightarrow E_{2}(Y)$ be a continuous linear operator which is positive. Furthermore, let $G$ be an arbitrary Banach space. Then

$$
A \otimes \mathrm{id}: E_{1}(X, G) \rightarrow E_{2}(Y, G)
$$

is bounded with $\|A \otimes \mathrm{id}\| \leq\|A\|$.

Proof. Since the step functions are dense in $E_{1}(X), A \otimes$ id is indeed an operator on $E_{1}(X, G)$ by proposition 2.6.12 and it is sufficient to prove boundedness for the step functions. In our representation of the step function

$$
\sum_{k=1}^{n} \chi_{M_{k}} x_{k}
$$


we may assume that the sets $M_{1}, \ldots, M_{n}$ are mutually disjoint. We then have:

$$
\begin{aligned}
\left\|(A \otimes \mathrm{id})\left(\sum_{k=1}^{n} \chi_{M_{k}} x_{k}\right)\right\| & =\|\| \sum_{k=1}^{n}\left(A \chi_{M_{k}}\right)(\cdot) x_{k}\left\|_{G}\right\|_{E_{2}} \\
& \leq\left\|\sum_{k=1}^{n} \mid\left(A \chi_{M_{k}}\right)(\cdot)\right\| x_{k}\left\|_{G}\right\|_{E_{2}} \\
& =\left\|\sum_{k=1}^{n}\left(A \chi_{M_{k}}\right)(\cdot)\right\| x_{k}\left\|_{G}\right\|_{E_{2}} \\
& =\left\|A\left(\sum_{k=1}^{n} \chi_{M_{k}}(\cdot)\left\|x_{k}\right\|_{G}\right)\right\|_{E_{2}} \\
& \leq\|A\|\left\|\sum_{k=1}^{n} \chi_{M_{k}}(\cdot)\right\| x_{k}\left\|_{G}\right\|_{E_{2}}
\end{aligned}
$$

Since the $M_{k}$ are mutually disjoint:

$$
\begin{aligned}
& =\|A\|\|\| \sum_{k=1}^{n} \chi_{M_{k}}(\cdot) x_{k}\left\|_{G}\right\|_{E_{2}} \\
& =\|A\|\left\|\sum_{k=1}^{n} \chi_{M_{k}} x_{k}\right\| .
\end{aligned}
$$

Combining the last two propositions, we get

Corollary 2.6.15. Let $E_{1}(X)$ and $E_{2}(Y)$ be Banach function spaces, where the step functions are dense in $E_{1}(X)$. Let $G_{1}, G_{2}$ be arbitrary Banach spaces. Finally, let $A_{2}: G_{1} \rightarrow G_{2}$ be a continuous linear operator, and $A_{1}: E_{1}(X) \rightarrow$ $E_{2}(Y)$ a continuous linear operator that is a linear combination of positive operators. Then

$$
A_{1} \otimes A_{2}: E_{1}\left(X, G_{1}\right) \rightarrow E_{2}\left(Y, G_{2}\right)
$$

is continuous, with

$$
\begin{aligned}
\left\|A_{1} \otimes A_{2}\right\| & \leq\left\|A_{2}\right\| \inf \left\{\sum_{k=1}^{n}\left|a_{k}\right|\left\|A_{1 k}\right\|:\right. \\
& \left.n \in \mathbb{N}, a_{1}, \ldots, a_{n} \in \mathbb{C}, A_{11}, \ldots, A_{1 k} \text { positive, } A_{1}=\sum_{k=1}^{n} a_{k} A_{1 k}\right\} .
\end{aligned}
$$

Corollary 2.6.16. Let $E_{1}(X)$ and $E_{2}(X)$ be Banach function spaces, where the step functions are dense in $E_{1}(X)$. Let $G$ be an arbitrary Banach space.

1. Let $g \in \mathfrak{M}(X)$ such that the linear operator

$$
A(f):=g f
$$


maps $E_{1}(X)$ continuously into $E_{2}(X)$. Then

$$
A \otimes \mathrm{id}: E_{1}(X, G) \rightarrow E_{2}(X, G)
$$

is continuous with

$$
\| A \otimes \text { id }\|\leq 4\| A \| .
$$

2. Let $H(X)$ be a Banach function space such that for all $g \in H(X)$ the operator

$$
A_{g}(f):=g * f
$$

maps $E_{1}(X)$ continuously into $E_{2}(X)$. Then all the operators

$$
A_{g} \otimes \text { id: } E_{1}(X, G) \rightarrow E_{2}(X, G)
$$

are continuous with

$$
\| A_{g} \otimes \text { id }\|\leq 4\| A_{|g|} \| .
$$

Proof. 1. Let $g_{1}, \ldots, g_{4} \in \mathfrak{M}^{+}(X)$ such that $g=g_{1}-g_{2}+i\left(g_{3}-g_{4}\right)$ and $\left|g_{i}\right| \leq$ $|g| \mu$-almost everywhere for $i=1, \ldots, 4$. Then also $\left|g_{i} f\right| \leq|g f| \mu$-almost everywhere. So by monotonicity, $g_{1}, \ldots, g_{4}$ define positive, continuous multiplication operators from $E_{1}(X)$ to $E_{2}(X)$ of norm smaller than or equal to $\|A\|$. This implies the claim.

2. By the equiabsoluteness property (B0), $g \in H(X)$ implies $|g| \in H(X)$. The operator $A_{|g|}$ is positive, and since $|g * f| \leq|g| *|f| \mu$-almost everywhere by the triangle inequality for integrals, we have $\left\|A_{g}\right\| \leq\left\|A_{|g|}\right\|$. The claim now follows again by decomposing $g$ into $g_{1}, \ldots, g_{4}$ as above.

\subsection{Other spaces}

In this section we shall very briefly introduce the Hardy spaces and the concept of Banach manifolds. We shall not work extensively with these structures and hence do not need to build a lot of theory around them. They are, however, important for directing this work to its goal. Hardy spaces are subspaces of $L^{1}$ but they are not Banach function spaces. Nevertheless, their relation to Bessel potential spaces will give the crucial hint which rearrangement-invariant function space is the "correct" one for our Schrödinger potentials. Finally, the parameter space of our Fermi moduli at the end of this work will be a quasiBanach manifold.

\subsubsection{Hardy spaces}

With respect to the Schrödinger operator, the following question is valid:

Question. For what $1 \leq p<\infty$ do $f \in L^{p}(F)$ and $\Delta f \in L^{p}(F)$ imply $f \in$ $W^{2, p}(F)$ ?

Let us, for a moment, transport this question to functions defined on $\mathbb{R}^{d}$. Given the nature of the Sobolev norm and considering one derivative at a 
time, this question generalises by proposition 2.1.6 to whether the operators $R_{1}, \ldots, R_{d}$ defined via the Fourier transform by

$$
\widehat{R_{i} f}=\frac{\kappa_{i}}{|\kappa|} \widehat{f}, \quad i=1, \ldots, d,
$$

(here, $\kappa$ runs through $\mathbb{R}^{d}$ due to our choice of basic measure space) map $L^{p}\left(\mathbb{R}^{d}\right)$ into itself. This is indeed the case for $1<p<\infty$ but not for $p=1$. We therefore define the Hardy space norm

$$
\|f\|_{H^{1}}:=\|f\|_{1}+\sum_{i=1}^{d}\left\|R_{i} f\right\|_{1}
$$

and the Hardy space

$$
H^{1}\left(\mathbb{R}^{d}\right):=\left\{f \in L^{1}\left(\mathbb{R}^{d}\right):\|f\|_{H^{1}}<\infty\right\} .
$$

With the Hardy space norm, the Hardy space is a Banach space. By construction, it is the optimal subspace $E$ of $L^{1}\left(\mathbb{R}^{d}\right)$ for which $f \in E$ and $\Delta f \in E$ imply $f \in W^{2,1}\left(\mathbb{R}^{d}\right)$.

There is another characterisation of $H^{1}\left(\mathbb{R}^{d}\right)$. A general reference for what follows is the Hardy space primer [Sem94. Let

$$
\mathfrak{T}:=\left\{g \in C^{\infty}\left(\mathbb{R}^{d}\right): \operatorname{supp} g \subseteq B_{1}(0),\|\nabla g\|_{\infty} \leq 1\right\} .
$$

Then we define the grand maximal function of a distribution $f$ :

$$
\begin{aligned}
& f \star \star \star \mathbb{R}^{d} \\
& x \mapsto[0, \infty], \\
& \sup _{0<t} \sup _{g \in \mathfrak{T}}\left|\int_{\mathbb{R}^{d}} t^{-d} f(x-y) g(y / t) \mathrm{d} y\right| .
\end{aligned}
$$

We now have

Theorem 2.7.1 (Maximal characterisation of $H^{1}\left(\mathbb{R}^{d}\right)$ ). Let $f$ be a distribution on $\mathbb{R}^{d}$. Then $f \in H^{1}\left(\mathbb{R}^{d}\right)$ if and only if $f_{\star \star}^{\star} \in L^{1}\left(\mathbb{R}^{d}\right)$. In fact, the grand maximal function induces a norm on $H^{1}\left(\mathbb{R}^{d}\right)$ which is equivalent to the Hardy norm.

Note that unlike the decreasing rearrangement $f^{\star}$ and the maximal function $f^{\star \star}$, the function $f_{\star \star}^{\star}$ is defined on $\mathbb{R}^{d}$ instead of on a subset of the real line. This is because, loosely speaking, that there is no rearrangement-invariance in the Hardy space, and no Luxemburg representation theorem. The relation between $f^{\star \star}$ and $f_{\star \star}^{\star}$ will become apparent soon. Also note that the definition of $\mathfrak{T}$ is somewhat arbitrary. Other definitions (bounds different from 1, rapidly decreasing instead of compactly supported functions, other orders of differentiation) lead to an equivalent norm.

Now that we have the Hardy space on $\mathbb{R}^{d}$, we should define it on $F$ as well. This is done by truncation of the grand maximal function. We omit the details (see [tr72, Gol79, Sem94]) and just mention that the norm is

$$
\|f\|_{H^{1}(F)}:=\int_{F} \sup _{0<t<\epsilon \in \sup }\left|\int_{\mathbb{R}^{d}} t^{-d} f(x-y) g(y / t) \mathrm{d} y\right| \mathrm{d} x,
$$


where $\epsilon>0$ is sufficiently small and depends only on $F$, and $F$ is identified with a fundamental domain in $\mathbb{R}^{d}$ ( $f$ is set to zero outside $F$ ).

The Hardy space $H^{1}(F)$ may be optimal for controlling the inverse of the Laplace operator, and it also fulfils $L^{p}(F) \subseteq H^{1}(F) \subseteq L^{1}(F)$ for all $1<p \leq \infty$, but it has one serious flaw: if $f \in H^{1}(F)$, then $|f| \notin H^{1}(F)$ in general. In particular, if $g \in L^{\infty}(F)$, then the operator $f \mapsto g f$ does not necessarily map $H^{1}(F)$ into itself. This also holds for continuous $g$. In fact, a true improvement on the exponent, such as $g$ being Hölder-continuous, is necessary for this simplest case of the Hölder inequality to hold. Since we are dealing with Schrödinger operators with potential, this failure of the equiabsoluteness property (B0) makes the Hardy space unusable for us. However, the following result of Stein Ste69, Theorem 2] gives us the crucial hint which space satisfying (B0) is the optimal replacement for the Hardy space.

Theorem 2.7.2. There is a constant $C>0$ depending only on $F$ such that for all $f \in L^{1,1 ; 1}(F)$

$$
\|f\|_{H^{1}} \leq C\|f\|_{1,1 ; 1}
$$

and for all $g \in H^{1}(F)$ with $g=|g| \mu$-almost everywhere

$$
\|g\|_{1,1 ; 1} \leq C\|g\|_{H^{1}} .
$$

In particular, the following inclusion relations hold:

$$
L^{1,1 ; 1}(F) \subseteq H^{1}(F), \quad \mathfrak{M}^{+}(F) \cap H^{1}(F) \subseteq L^{1,1 ; 1}(F) .
$$

This immediately gives us the following corollary:

Corollary 2.7.3. Let $E$ be any normed space of measurable functions on $F$ such that

1. $f \in E$ implies $|f| \in E$, and $\|\cdot\|$ and $\||\cdot|\|$ are equivalent norms on $E$,

2. $E$ is continuously embedded in the Hardy space $H^{1}(F)$.

Then $E$ is continuously embedded in the Lorentz-Zygmund space $L^{1,1 ; 1}(F)$.

Proof. If $f \in E$, then $|f| \in E$ by the first condition, so by theorem 2.7.2. $|f| \in L^{1,1 ; 1}(F)$ and thus $f \in L^{1,1 ; 1}(F)$ since $L^{1,1 ; 1}(F)$ fulfils (B0). Obviously, all these mappings are continuous.

This means that in terms of function spaces on $F$, the Lorentz-Zygmund space $L^{1,1 ; 1}(F)$ is the optimal space $E$ fulfilling the following two properties:

1. If $f \in E$ and $\Delta f \in E$, then $f \in W^{2,1}(F)$.

2. For all $g \in L^{\infty}(F)$, the operator $f \mapsto g f$ maps $E$ boundedly into itself.

The relation between $f^{\star \star}$ and $f_{\star \star}^{\star}$ promised above now becomes apparent by comparing proposition 2.4.14 with theorem 2.7.1. 


\subsubsection{Banach manifolds}

In the course of investigating the asymptotic behaviour of Schrödinger spectra, we shall, near the end of this thesis, encounter structures which cannot be identified with a Banach space on a global scale. Locally however, they are, in an appropriate sense, isomorphic to some open subset of a Banach space. Therefore, it makes sense to use the manifold concept in order to model these structures. Hence, Banach manifolds, as we shall introduce them momentarily, are one possibility of generalising the well-known finite-dimensional manifolds to an infinite number of dimensions.

Let $M$ be some Hausdorff space and $E$ be some Banach space. Assume that for each $x \in M$, there is an open neighbourhood $U_{x} \subseteq M$ and an injective function $\varphi_{x}: U_{x} \rightarrow E$ whose image is an open subset of $E$. Assume further that for each $x, y \in M$, the composition maps

$$
\varphi_{y} \circ \varphi_{x}^{-1}: \varphi_{x}\left(U_{x} \cap U_{y}\right) \rightarrow \varphi_{y}\left(U_{x} \cap U_{y}\right)
$$

fulfil some regularity requirement that is to be specified together with the manifold. Typical choices are continuity, differentiability, smoothness, or even analyticity (all of these are possible between two Banach spaces). Then $M$, together with the choices $U_{x}$ and $\varphi_{x}$ is called an E-Banach manifold of the specified regularity.

The choices of $U_{x}$ and $\varphi_{x}$ are not necessarily unique, giving rise to the well-known concepts of different atlases and atlas equivalence. However, as an interesting sidenote, we remark that all infinite-dimensional continuous separable Banach manifolds can be embedded as open subsets into the (unique up to isomorphism) separable Hilbert space [Hen69].

\subsection{Localisation of function space norms}

In this section, we shall define new norms for certain classes of Banach function spaces which are equivalent to the standard norms. These norms will be helpful in altering the bounds of specific linear operators between Banach function spaces later on.

\subsubsection{Localisation on the torus}

Definition 2.8.1. Let $E$ be a quasi-Banach function space and $f \in E$. The function $f$ is called absolutely continuous in $E$ if

$$
\lim _{n \rightarrow \infty}\left\|f \chi_{A_{n}}\right\|=0
$$

for all decreasing sequences of measurable sets $\left(A_{n}\right)_{n \in \mathbb{N}}$ such that

$$
\lim _{n \rightarrow \infty} \chi_{A_{n}}(x)=0
$$

for $\mu$-almost all $x \in X$. If all $f \in E$ are absolutely continuous, then $E$ itself is called absolutely continuous.

Most Lorentz-Zygmund spaces are absolutely continuous: 
Theorem 2.8.2. The Lorentz-Zygmund space $L^{p, a ; \alpha}(X)$ with $0<a<\infty$ is absolutely continuous.

Proof. The space $L^{1}(X)$ is clearly absolutely continuous. Now,

$$
\lim _{n \rightarrow \infty}\left(f \chi_{A_{n}}\right)^{\star}(t)=0
$$

pointwise for $t>0$, so $\left\|f \chi_{A_{n}}\right\|_{p, a ; \alpha} \rightarrow 0$ for a decreasing sequence of sets converging to a set of zero measure.

Note that this theorem does not hold for $a=\infty$ because in this case the Lorentz-Zygmund norm is defined via a supremum. In particular, $L^{\infty}(X)$ is not absolutely continuous.

Before we can proceed, we need some discrete geometry. In particular, we shall define a family of geometric coverings of $F$. Recall the ordered basis $B=$ $\left(b_{1}, \ldots, b_{d}\right)$ for the lattice $\Gamma$. In the fundamental domain interpretation of $F$, every $x \in F$ is uniquely determined as implied by the representation

$$
F=\bigcup_{i=1}^{d} b_{i}[0,1)
$$

Clearly,

$$
U_{*}:=\bigcup_{i=1}^{d} b_{i}(-1,1)
$$

is an open set covering $F$. Let $\mathcal{U}_{0}$ be the family containing only $U_{*}$. Now let $a^{1} \in\{0,1\}^{d}$ and set

$$
U_{a^{1}}:=\left\{\sum_{i=1}^{d} b_{i} x_{i} \in U_{*}: 0<x_{i}+a_{i}^{1} / 2<1 \text { for all } i=1, \ldots, d\right\} .
$$

Again, the family $\mathcal{U}_{1}:=\left\{U_{a^{1}}\right\}_{a^{1} \in\{0,1\}^{d}}$ covers $F$ with $2^{d}$ congruent open sets. We can continue this construction recursively. Assume $\mathcal{U}_{n-1}$ is an open cover of the $2^{(n-1) d}$ open sets $U_{a^{1} a^{2} \cdots a^{n-1}}$ with $a^{1}, \ldots, a^{n-1} \in\{0,1\}^{d}$, then for $a^{n} \in$ $\{0,1\}^{d}$ we set

$$
\begin{aligned}
U_{a^{1} a^{2} \cdots a^{n}}:=\left\{\sum_{i=1}^{d} b_{i} x_{i}\right. & \in U_{a^{1} a^{2} \ldots a^{n-1}}: \\
& \left.1-2^{1-n}<x_{i}+\sum_{k=1}^{n} a_{i}^{k} 2^{-k}<1 \text { for all } i=1, \ldots, d\right\},
\end{aligned}
$$

creating a new open cover $\mathcal{U}_{n}$ containing $2^{\text {nd }}$ congruent sets. The sequence $\left(\mathcal{U}_{n}\right)_{n \in \mathbb{N}}$ corresponds to a sequence $\left(\mathcal{F}_{n}\right)_{n \in \mathbb{N}}$ of partitions, where $\mathcal{F}_{0}:=\{F\}$ and $\mathcal{F}_{n}$ consists of the congruent sets

$$
\begin{aligned}
F_{a^{1} a^{2} \cdots a^{n}}:=\left\{\sum_{i=1}^{d} b_{i} x_{i}\right. & \in F_{a^{1} a^{2} \cdots a^{n-1}}: \\
& \left.1-2^{-n} \leq x_{i}+\sum_{k=1}^{n} a_{i}^{k} 2^{-k}<1 \text { for all } i=1, \ldots, d\right\} .
\end{aligned}
$$


In particular, for every $n \in \mathbb{N}$ and every $x \in F$ there are unique $a^{1}, \ldots, a^{n}$ such that $x \in F_{a^{1} \ldots a^{n}}$. Hence, $x \in U_{a^{1} \cdots a^{n}}$ though the latter set is not unique.

Lemma 2.8.3. Let $n \in \mathbb{Z}_{\geq 0}, U \in \mathcal{U}_{n+1}$ and $U_{1}, U_{2} \in \mathcal{U}_{n}$ such that $U \subseteq U_{1} \cup U_{2}$. Then $U \subseteq U_{1}$ or $U \subseteq U_{2}$.

Proof. Let $a^{1}, \ldots, a^{n+1}, b^{1}, \ldots, b^{n}, c^{1}, \ldots, c^{n} \in\{0,1\}^{d}$ such that $U=U_{a^{1} \ldots a^{n+1}}$, $U_{1}=U_{b^{1} \ldots b^{n}}$ and $U_{2}=U_{c^{1} \ldots c^{n}}$. Then, for all $x \in U$, the inequality

$$
1-2^{-n}<x_{i}+\sum_{k=1}^{n+1} a_{i}^{k} 2^{-k}<1
$$

and one of the inequalities

$$
1-2^{1-n}<x_{i}+\sum_{k=1}^{n} b_{i}^{k} 2^{-k}<1, \quad 1-2^{1-n}<x_{i}+\sum_{k=1}^{n} c_{i}^{k} 2^{-k}<1
$$

is fulfilled for all $i=1, \ldots, d$. (Which one depends only on $x$, not on $i$.) Now assume the lemma is false. Then there exists an $x^{0} \in U$ which fulfils both inequalities 2.8.5 since $U$ is connected. Since finite binary expansions represent a unique rational number, 2.8.5 implies $\left|\left(b_{i}^{1} \cdots b_{i}^{n}\right)_{2}-\left(c_{i}^{1} \cdots c_{i}^{n}\right)_{2}\right| \leq 1$ for all $i=1, \ldots, d$. Now, for an arbitrary $i$, we can have the following cases: if $\left(b_{i}^{1} \cdots b_{i}^{n}\right)_{2}=\left(c_{i}^{1} \cdots c_{i}^{n}\right)_{2}$ then by $(2.8 .4)$, both inequalities 2.8.5) are trivially fulfilled by all $x \in U$. If $\left(b_{i}^{1} \cdots b_{i}^{n}\right)_{2}<\left(c_{i}^{1} \cdots c_{i}^{n}\right)_{2}$, the difference is 1 , so we get

$$
1-2^{-n}<x_{i}^{0}+\sum_{k=1}^{n} c_{i}^{k} 2^{-k}<1 .
$$

This implies $\left|\left(a_{i}^{1} \cdots a_{i}^{n+1}\right)_{2}-\left(c_{i}^{1} \cdots c_{i}^{n} 0\right)_{2}\right| \leq 1$. If $a_{i}^{n+1}=0$ then necessarily $c_{i}^{k}=a_{i}^{k}$ for all $k=1, \ldots, n$ and again, both inequalities (2.8.5) are fulfilled for all $x \in U$. The same holds for $a_{i}^{n+1}=1$ and $\left(a_{i}^{1} \cdots a_{i}^{n}\right)_{2}+1=\left(c_{i}^{1} \cdots c_{i}^{n}\right)_{2}$. Now, if $a_{i}^{n+1}=1$ and $c_{i}^{k}=a_{i}^{k}$ for all $k=1, \ldots, n$, then all $x \in U$ fulfil the second inequality of 2.8.5). But some $x \in U$, such as the centre point $\dot{x}$, that is the point such that

$$
\dot{x}_{i}+\sum_{k=1}^{n+1} a_{i}^{k} 2^{-k}=1-2^{-n-1}
$$

for all $i=1, \ldots, d$, do not fulfil the first inequality of 2.8.5. The case $\left(b_{i}^{1} \cdots b_{i}^{n}\right)_{2}>\left(c_{i}^{1} \cdots c_{i}^{n}\right)_{2}$ works similarly, only that $b$ and $c$ are swapped. In particular, in the problematic case $a_{i}^{n+1}=1$ and $b_{i}^{k}=a_{i}^{k}$ for all $k=1, \ldots, n$, this time the centre point $\dot{x}$ fulfils the first of the equalities 2.8.5 but not the second. The above case-by-case analysis depends only on $a, b, c$ but not on $x$. On the other hand, the centre point must fulfil one or the other inequality of (2.8.5), independently of $i$. This means that for any distinct $i, j$, the case that $a_{i}^{n+1}=a_{j}^{n+1}=1,\left(b_{i}^{1} \cdots b_{i}^{n}\right)_{2}<\left(c_{i}^{1} \cdots c_{i}^{n}\right)_{2}, a_{i}^{k}=c_{i}^{k},\left(b_{j}^{1} \cdots b_{j}^{n}\right)_{2}>\left(c_{j}^{1} \cdots c_{j}^{n}\right)_{2}$ and $a_{j}^{k}=b_{j}^{k}$, for all $k=1, \ldots, n$, never occurs. In total this implies $U \subseteq U_{1}$ or $U \subseteq U_{2}$, contrary to our assumption. Hence, the lemma must be true.

Lemma 2.8.6. Let $n \in \mathbb{N}, x \in F$ and $r>0$ such that the ball $B_{r}(x)$ is contained in a parallel displacement of one of the congruent sets in $\mathcal{U}_{n+1}$. Furthermore, let

$$
\mathcal{V} \subseteq \bigcup_{k=0}^{n} \mathcal{U}_{k}
$$


be a cover of $F$. Then there is a $V \in \mathcal{V}$ such that $B_{r}(x) \subseteq V$.

Proof. By construction, each parallel displacement of a set in $\mathcal{U}_{n+1}$ is contained in a set $V^{\prime} \in \mathcal{U}_{n}$, so all we need to show is that there is a $V \in \mathcal{V}$ such that $V^{\prime} \subseteq V$. The set

$$
\mathfrak{U}:=\bigcup_{k=0}^{n} \mathcal{U}_{k}
$$

is partially ordered with respect to inclusion. By construction, the order graph of $\mathfrak{U}$ is directed, connected, has a unique maximum, namely $U_{*}$, which we call the root, and $2^{\text {nd }}$ minima, namely the elements in $\mathcal{U}_{n}$, which we call the leaves ${ }^{12}$. We shall now colour the vertices of this graph in red or black as follows. We may assume $\mathcal{V}$ to be a minimal cover. For each $U \in \mathcal{V}$, we colour the respective vertex red, and all other vertices on a path from the root to a leaf through $U$ we colour black. Since $\mathcal{V}$ is minimal, no vertex ends up with both red and black colour. Since $\mathcal{V}$ is a cover, all leaves (and hence all other vertices) are coloured. Since $V^{\prime}$ is a leaf, there is a red vertex on some path from $U_{*}$ to $V^{\prime}$. This red vertex corresponds to the $V \in \mathcal{V}$ we seek.

Now we can prove

Proposition 2.8.7. Let $E$ be a Banach function space on the measure space $F$ and let $f \in E$ be absolutely continuous. Then

$$
\lim _{r \rightarrow 0} \sup _{x \in F}\left\|f \chi_{B_{r}(x)}\right\|=0 .
$$

Proof. Let $\epsilon>0$. For each $x \in F$ there is a unique sequence $\left(F_{n}^{x}\right)_{n \in \mathbb{N}}$ with $F_{n}^{x} \in \mathcal{F}_{n}$ for all $n \in \mathbb{N}$ which is decreasing and converges to $\{x\}$. To this sequence there corresponds a sequence $\left(U_{n}^{x}\right)_{n \in \mathbb{N}}$ with $U_{n}^{x} \in \mathcal{U}_{n}$ for all $n \in \mathbb{N}$ which by construction also converges to $\{x\}$. Since $\mu(\{x\})=0$ for all $x \in F$ and since $f$ is absolutely continuous, there is an $n_{x} \in \mathbb{N}$ for each $x \in F$ such that $\left\|f \chi_{U_{n_{x}}^{x}}\right\|<\epsilon$. The family $\left\{U_{n_{x}}^{x}\right\}_{x \in F}$ is an open cover of $F$. Since $F$ is compact, there is a finite subcover $\left\{U_{n_{k}}^{k}\right\}_{k=1}^{N}$. Let

$$
n:=\min _{k=1}^{N} n_{k}
$$

and choose $r>0$ sufficiently small such that any ball of radius $r$ is contained in a parallel displacement of a set from $\mathcal{U}_{n+1}$. Then $\left\|f \chi_{B_{r}(x)}\right\|<\epsilon$ for all $x \in F$ by lemma 2.8.6. This implies the claim.

Definition 2.8.8. Let $r_{1}>r_{2}>0$. We denote by $\nu_{r_{1}, r_{2}}$ the minimum number of balls of radius $r_{2}$ required to cover a single ball of radius $r_{1}$ in $\mathbb{R}^{d}$.

Lemma 2.8.9. Let $r>0$, then $\nu_{r, r / 2}$ depends only on $d$.

Proof. Clearly, $\nu_{1,1 / 2}$ depends only on $d$. Scaling does not alter the covering geometry, so the lemma follows.

We can now define a family of equivalent norms for absolutely continuous Banach spaces on $F$. This localisation scheme is a very simple variation on the idea of Kato classes Kat72 (cf. proposition 2.8.7).

\footnotetext{
${ }^{12}$ Despite this terminology, $\mathfrak{U}$ is not a tree because a branch may split and then grow back together again.
} 
Definition 2.8.10. Let $r>0$ and let $E$ be a Banach space of functions on $F$ with norm $\|\cdot\|$. For each $x \in F$, denote the characteristic function of $B_{r}(x)$ by $\chi_{r}^{x}$. Then we define the $r$-localised norm (or sometimes simply the localised norm) $\|\cdot\|_{r}$ by

$$
\|f\|_{r}:=\sup _{x \in F}\left\|f \chi_{r}^{x}\right\| .
$$

Theorem 2.8.11. Let $E$ be a Banach function space on $F$ with norm $\|\cdot\|$. Then for all $r>0$, the localised norm $\|\cdot\|_{r}$ is a norm on E. Moreover, for $r_{1}>r_{2}>0$ we have

$$
\frac{1}{\nu_{r_{1}, r_{2}}}\|f\|_{r_{1}} \leq\|f\|_{r_{2}} \leq\|f\|_{r_{1}} .
$$

In particular, $\|\cdot\|$ and $\|\cdot\|_{r}$ are equivalent for all $r>0$ and $E$ is complete with respect to these norms.

Proof. Properties (F1) through (F3) for a norm a clear. For the triangle inequality we have

$$
\|f+g\|_{r}=\sup _{x \in F}\left\|f \chi_{r}^{x}+g \chi_{r}^{x}\right\| \leq \sup _{x \in F}\left(\left\|f \chi_{r}^{x}\right\|+\left\|g \chi_{r}^{x}\right\|\right) \leq\|f\|_{r}+\|g\|_{r} .
$$

Therefore, $\|\cdot\|_{r}$ is a norm for all $r>0$. Now we prove equivalence. The second inequality immediately follows from the monotonicity property (B6). For the first inequality, let $x_{1}, \ldots, x_{\nu_{r_{1}, r_{2}}} \in F$ be such that $\left\{B_{r_{2}}\left(x_{i}\right)\right\}_{i=1}^{\nu_{r_{1}, r_{2}}}$ is a cover of $B_{r_{1}}(0)$ and let $M_{1}, \ldots, M_{\nu_{r_{1}, r_{2}}}$ be a partition of $B_{r_{1}}(0)$ such that $M_{i} \subseteq B_{r_{2}}\left(x_{i}\right)$ for all $i=1, \ldots, \nu_{r_{1}, r_{2}}$. Then we have for all $x \in F$

$$
\left\|f \chi_{r_{1}}^{x}\right\|=\left\|\sum_{i=1}^{\nu_{r_{1}, r_{2}}} f \chi_{x+M_{i}}\right\| \leq \sum_{i=1}^{\nu_{r_{1}, r_{2}}}\left\|f \chi_{x+M_{i}}\right\|
$$

By the monotonicity property (B6):

$$
\leq \sum_{i=1}^{\nu_{r_{1}, r_{2}}}\left\|f \chi_{r_{2}}^{x+x_{i}}\right\| \leq \nu_{r_{1}, r_{2}} \sup _{x \in F}\left\|f \chi_{r_{2}}^{x}\right\| .
$$

This shows the first inequality and hence the equivalence of $\|\cdot\|_{r_{1}}$ and $\|\cdot\|_{r_{2}}$. It also shows the equivalence of these norms with $\|\cdot\|$ as we may choose $r>0$ sufficiently large so that $F \subseteq B_{r}(x)$ for all $x \in F$. Completeness now follows from equivalence.

A few remarks are in order. First, note that generally the constant $\nu_{r_{1}, r_{2}}^{-1}$ is by no means optimal. Secondly, note that this theorem holds for all Banach function spaces on $F$. However, it is only of interest for us where proposition 2.8.7 holds. For example, on $L^{\infty}(F)$ the resultant norms are all equal. Finally, the Sobolev spaces $W^{m, p}(F)$ are not Banach function spaces but since the Sobolev norms are defined in terms of Lebesgue norms we can define localised norms for Sobolev spaces simply by setting

$$
\|f\|_{m, p ; r}:=\sum_{|\alpha| \leq m}\left\|\partial^{\alpha} f\right\|_{p ; r} .
$$




\subsubsection{Localisation on discrete lattices}

Now that we have developed a localisation scheme for functions on $F$, we shall do likewise for sequences on $\Gamma^{*}$. In analogy with absolute continuity, we introduce the following concept.

Definition 2.8.12. Let $E$ be a Banach function space and let $f \in E$ be such that $f$ is the limit (in $E$ ) of a sequence of step functions. Then $f$ is called simply generated. If all $f \in E$ are simply generated, then $E$ is called simply generated.

Actually, this is a weaker property than absolute continuity.

Theorem 2.8.13. Every absolutely continuous Banach function space is simply generated.

Proof. BS88, Theorem 1.3.11].

However, we shall not need simply generated Banach function spaces in cases where this property is actually strictly weaker than absolute continuity (in fact, this is never the case when $X=\Gamma^{*}$, see [BS88, Theorem 1.3.13]). Rather, the need for a different approach stems from the atomicity of $\Gamma^{*}$. In particular, proposition 2.8.7 does not hold for sequences on $\Gamma^{*}$. Therefore, we shall introduce a different localisation scheme, for which the concept of simply generated functions is better suited than absolute continuity. In particular, we have the following proposition:

Proposition 2.8.14. Let $E$ be a Banach function space on $\Gamma^{*}$ and let $f \in E$ be simply generated. Then for each increasing sequence $\left(A_{n}\right)_{n \in \mathbb{N}}$ of finite subsets exhausting $\Gamma^{*}$

$$
\lim _{n \rightarrow \infty}\left\|f_{A_{n}}\right\|=0
$$

where $f_{A_{n}}$ is the function defined by

$$
\begin{aligned}
f_{A_{n}}: \Gamma^{*} & \rightarrow \mathbb{C}, \\
\kappa & \mapsto \begin{cases}0, & \kappa \in A_{n}, \\
f(\kappa), & \kappa \notin A_{n} .\end{cases}
\end{aligned}
$$

Proof. Let $\left(f_{m}\right)_{m \in \mathbb{N}}$ be a sequence of step functions in $E$ converging to $f$. We may assume these step functions have increasing supports $B_{m}$. Since these supports are finite and $\left(A_{n}\right)_{n \in \mathbb{N}}$ exhausts $\Gamma^{*}$, for each $m \in \mathbb{N}$ there is some $N \in \mathbb{N}$ such that for each $n \geq N, B_{m} \subseteq A_{n}$. Hence, $\left\|f_{A_{n}}\right\| \leq\left\|f-f_{m}\right\|$ by the monotonicity property. Now,

$$
\lim _{m \rightarrow \infty}\left\|f-f_{m}\right\|=0
$$

by construction. This proves the claim.

As in the case of absolutely continuous Banach function spaces on $F$, we now define a family of equivalent norms on a simply generated Banach function space on $\Gamma^{*}$. 
Definition 2.8.15. Let $A \subseteq \Gamma^{*}$ be finite and let $E$ be a Banach space of functions on $\Gamma^{*}$ with norm $\|\cdot\|$. Then we define the A-localised norm (or sometimes simply the localised norm) $\|\cdot\|_{A}$ by

$$
\|f\|_{A}:=\left\|f_{A}\right\|+\frac{1}{\mu(A)}\left\|f-f_{A}\right\|_{\infty} .
$$

Theorem 2.8.16. Let $E$ be a Banach function space over $\Gamma^{*}$ with norm $\|\cdot\|$. Then for all finite $A \subseteq \Gamma^{*}$, the A-localised norm $\|\cdot\|_{A}$ is a norm on $E$. Moreover, all these norms are equivalent to $\|\cdot\|$, and $E$ is complete with respect to these norms.

Proof. Positivity and regularity of $\|\cdot\|_{A}$ are clear. Absolute linearity follows since $(\lambda f)_{A}=\lambda f_{A}$ for all $\lambda \in \mathbb{C}$. For the triangle inequality, we have

$$
\begin{aligned}
\|f+g\|_{A} & =\left\|(f+g)_{A}\right\|+\frac{1}{\mu(A)}\left\|(f+g)-(f+g)_{A}\right\|_{\infty} \\
& =\left\|f_{A}+g_{A}\right\|+\frac{1}{\mu(A)}\left\|f-f_{A}+g-g_{A}\right\|_{\infty} \\
& \leq\left\|f_{A}\right\|+\left\|g_{A}\right\|+\frac{1}{\mu(A)}\left\|f-f_{A}\right\|_{\infty}+\frac{1}{\mu(A)}\left\|g-g_{A}\right\|_{\infty}=\|f\|_{A}+\|g\|_{A}
\end{aligned}
$$

for $f, g \in E$. As for equivalence, we have, on the one hand,

$$
\|f\|=\left\|f_{A}+f-f_{A}\right\| \leq\left\|f_{A}\right\|+\left\|f-f_{A}\right\| .
$$

Now, the support of $f-f_{A}$ is the finite set $A$, so the underlying vector space of functions is finite-dimensional. There, the norms $\|\cdot\|$ and $\|\cdot\|_{\infty}$ are equivalent. (In particular, the expression $\left\|f-f_{A}\right\|_{\infty}$ always yields a finite value.) On the other hand, we have due to equivalence in finite dimensions and due to the monotonicity property

$$
\begin{aligned}
\|f\|_{A}=\left\|f_{A}\right\|+\frac{1}{\mu(A)}\left\|f-f_{A}\right\|_{\infty} & \leq\left\|f_{A}\right\|+C\left\|f-f_{A}\right\| \\
& \leq\left\|f_{A}\right\|+C\|f\|+C\left\|f_{A}\right\| \leq(2 C+1)\|f\|
\end{aligned}
$$

for some $C>0$ depending only on $E$ and $\mu(A)$. Hence, all localised norms are equivalent to $\|\cdot\|$. This also implies completeness of the localised norms.

Thus we have developed a localisation scheme for certain function spaces on the measure space $\Gamma^{*}$ in analogy to the $r$-localised norms on certain function spaces on $F$. However, the $A$-localised norms have a major drawback in comparison with their $r$-localised counterparts: $A$-localised norms are not translation invariant. Since translation invariance will play some role later on, we now generalise the $A$-localised norms to a family of quasi-norms which are translation invariant. For the sake of simplicity, we shall do so only for the Lorentz space $\ell^{\infty, 1}\left(\Gamma^{*}\right)$, though the principles developed admit easy generalisation to many other spaces. Recall that the norm for some $f \in \ell^{\infty, 1}\left(\Gamma^{*}\right)$ is given by

$$
\|f\|_{\infty, 1}=\sum_{n=1}^{\infty} \frac{f^{\star}(n)}{n} .
$$

We now introduce the following family of quasi-norms on $\ell^{\infty, 1}\left(\Gamma^{*}\right)$ : 
Definition 2.8.17. Let $f \in \ell^{\infty, 1}\left(\Gamma^{*}\right)$ and $N \in \mathbb{N}$. Then we define the $N$ localised quasi-norm

$$
\|f\|_{(\infty, 1) ; N}:=\frac{f^{\star}(1)}{N}+\sum_{n=1}^{\infty} \frac{f^{\star}(N+n)}{n} .
$$

We then have the following analogon of proposition 2.8 .7

Proposition 2.8.18. Let $f \in \ell^{\infty, 1}\left(\Gamma^{*}\right)$, then

$$
\lim _{N \rightarrow \infty}\|f\|_{(\infty, 1) ; N}=0 .
$$

Proof. Let $A_{N} \subseteq \Gamma^{*}$ be the support of the $N$ largest absolute values of $f$. By consistent choice of the $A_{N}$ and possibly enlarging them with some elements where $f$ is zero, we can make $\left(A_{N}\right)_{N \in \mathbb{N}}$ an increasing sequence of finite sets exhausting $\Gamma^{*}$. The claim now follows from proposition 2.8.14.

So actually, the $N$-localised quasi-norms are a simple generalisation of the $A$ localised norms with suitable $A$ because $\|f\|_{\infty}=f^{\star}(1)$ and we may assume $N=$ $\mu(A)$. The difference here (which also destroys the triangle inequality) is the dependence of $A$ on $f$. Note that, again, convergence in proposition 2.8.18 is not uniform.

We can now prove

Theorem 2.8.19. For all $N \in \mathbb{N}$, the functionals $\|\cdot\|_{(\infty, 1) ; N}$ are quasi-norms equivalent to $\|\cdot\|_{\infty, 1}$. Furthermore, the quasi-norms $\|\cdot\|_{(\infty, 1) ; N}$ fulfil the triangle inequality for those $f, g \in \ell^{\infty, 1}\left(\Gamma^{*}\right)$ whose support of the $N$ largest absolute values may be assumed to be identical.

Proof. The functionals $\|\cdot\|_{(\infty, 1) ; N}$ obviously fulfil (F1) through (F3). In order to show that they are quasi-norms, it is sufficient to show equivalence with $\|\cdot\|_{\infty, 1}$. So let $f \in \ell^{\infty, 1}\left(\Gamma^{*}\right)$. Then clearly $\|f\|_{(\infty, 1) ; N} \leq\|f\|_{\infty, 1}$ for all $N \in \mathbb{N}$. As for the reverse inequality, we have

$$
\|f\|_{\infty, 1}=\sum_{n=1}^{\infty} \frac{f^{\star}(n)}{n} \leq N f^{\star}(1)+\sum_{n=1}^{\infty} \frac{f^{\star}(N+n)}{N+n} \leq N^{2}\|f\|_{(\infty, 1) ; N} .
$$

The triangle inequality for suitable $f, g \in \ell^{\infty, 1}\left(\Gamma^{*}\right)$ follows as in the proof of theorem 2.8.16.

\subsubsection{Translation and levelling operators}

In indirect relationship with localised norms on rearrangement-invariant Banach function spaces on $\Gamma^{*}$ used together with a certain convolution operation, we shall later encounter expressions which arise through "levelling" of function values. Therefore, we shall very briefly investigate the behaviour of the operators involved.

Definition 2.8.20. Let $T$ be an endomorphism of the space of functions $\Gamma^{*} \rightarrow \mathbb{C}$ for which there is a $\nu \in \Gamma^{*}$ such that the equation

$$
(T f)(\kappa)=f(\nu+\kappa)
$$

is fulfilled for all $f: \Gamma^{*} \rightarrow \mathbb{C}$ and all $\kappa \in \Gamma^{*}$. Then $T$ is called a translation operator. 
Proposition 2.8.21. Let $E$ be a rearrangement-invariant function space on $\Gamma^{*}$ and let $T$ be a translation operator. Then $T$ is a continuous linear operator on $E$ with norm 1.

Proof. It is clear that $T$ is linear. Let $f \in E$. Recall that $f^{\star}$ is the sequence of values of $f$ in decreasing order by absolute value. Now, $T$ is a translation operator, so $(T f)^{\star}=f^{\star}$. Since $E$ is rearrangement-invariant, it follows that $T f \in E$ and $\|T f\|=\|f\|$. Therefore, $\|T\|=1$.

Definition 2.8.22. Let $T_{1}, \ldots, T_{n}$ be translation operators. Then the operator

$$
\Lambda:=\frac{1}{n} \sum_{k=1}^{n} T_{k}
$$

is called a levelling operator.

The name "levelling operator" may seem somewhat odd and is chosen for lack of a better one. The term "averaging operator" is already taken for a slightly different concept, and "mollifier" is inappropriate for the discrete space $\Gamma^{*}$.

Proposition 2.8.23. Let $E$ be a rearrangement-invariant space over $\Gamma^{*}$ and let $\Lambda$ be a levelling operator. Then $\Lambda$ is a continuous linear operator on $E$ with norm at most one.

Proof. This follows immediately from proposition 2.8.21 and the triangle inequality.

Note that for quasi-norms instead of norms, levelling operators are still continuous, but the bound may be larger than one. 


\section{Chapter 3}

\section{Schrödinger operators}

In this chapter we shall introduce Schrödinger operators with periodic potentials and quasi-periodic boundary conditions. In doing so, we will apply a standard procedure to hide the quasiness of the periodicity, so we need only consider functions on the torus $F$.

In order to apply spectral methods to our Schrödinger operators, we need a good analytical description of their resolvents. We shall develop such a description for the free Schrödinger operator first and then generalise our results to the remaining Schrödinger operators. Here, the Sobolev and Lorentz-Zygmund spaces introduced in the previous chapter will be the right tool. The theory developed in this chapter will enable us to establish the Schrödinger resolvents in chapter 4 as meromorphic families of compact operators on $L^{2}(F)$.

\subsection{Schrödinger operators on the torus}

By the Schrödinger equation we shall mean its time-independent variant

$$
(-\Delta+u) \psi=\lambda \psi
$$

We had already mentioned the Laplace operator $\Delta$ in section 2.2 The potential $u$ is some function $\mathbb{R}^{d} \rightarrow \mathbb{C}$, as is the solution $\psi$. The number $\lambda \in \mathbb{C}$ is called the eigenvalue with respect to a non-zero solution $\psi$. In the directly stated (non-inverse) problem, $u$ is generally assumed to be known, while $\lambda$ and $\psi$ are sought. The selection of $u$ and $\psi$ should be restricted to spaces where the Schrödinger equation (3.1.1) is, in a suitable sense, well-defined. We shall become very specific on this topic in the following two sections.

Let us now define our boundary conditions for the Schrödinger equation. First, we assume the potential $u$ to be periodic, that is, there is a non-degenerate geometric lattice $\Gamma \subseteq \mathbb{R}^{d}$ (see section 2.1) such that

$$
u(x+\gamma)=u(x) \text { for all } x \in \mathbb{R}^{d}, \gamma \in \Gamma .
$$

We also assume the solution $\psi$ to be quasi-periodic with respect to the same lattice $\Gamma$. That is, there is some boundary condition $k \in \mathbb{C}^{d}$ such that

$$
\psi(x+\gamma)=e^{2 \pi i(k \mid \gamma)} \psi(x) \quad \text { for all } x \in \mathbb{R}^{d}, \gamma \in \Gamma \text {. }
$$


Equations (3.1.1), 3.1.2 and (3.1.3 make up the periodic Schrödinger equation with quasi-periodic boundary conditions. This equation may be used to approximately describe the situation effective in a crystalline solid. Note, however, that our Schrödinger equation takes neither magnetic fields (in form of a vector potential) nor relativistic effects into account.

Technically, the periodicity of $u$ is not part of the boundary conditions because it affects the Schrödinger operator $-\Delta+u$ itself. But we shall conflate these notions now anyhow. The periodicity of $u$ allows us to consider $u$ to be a function on the torus $F=\mathbb{R}^{d} / \Gamma$ instead of on $\mathbb{R}^{d}$. It would be very convenient to be able to formulate the entire problem on $F$ instead of on $\mathbb{R}^{d}$. Unfortunately, the solution $\psi$ makes trouble because in general, it is only quasi-periodic $(k=0$ would be an obvious exception). The standard procedure (see e.g. [KT90]) here is to "hide" the boundary condition in the Laplace operator. To this end, we define for all $k \in \mathbb{C}^{d}$ :

$$
\Delta_{k}:=\nabla_{k}^{2}:=(\nabla+2 \pi i k)^{2} .
$$

We use this notation as a shorthand for the the operator $f \mapsto(\nabla+2 \pi i k)((\nabla+$ $2 \pi i k) f)$, where the first $(\nabla+2 \pi k)$ maps the scalar function $f$ to a vector-valued quantity, while the second one maps it back to a scalar function. A physicist might baptise the first (rightmost) operator $\operatorname{grad}_{k}$, and the second operator $\operatorname{div}_{k}$. We shall abuse notation further and extend the Euclidean dot product $(\cdot \mid \cdot)$ to any quantity where it makes sense. For example, we write

$$
(k \mid \nabla)=\sum_{i=1}^{d} k_{i} \partial_{i} .
$$

With this notation, we can also express $\Delta_{k}$ more directly as a scalar operator:

Lemma 3.1.5. For all $k \in \mathbb{C}^{d}$ we have

$$
\Delta_{k}=\Delta+4 \pi i(k \mid \nabla)-4 \pi^{2} k^{2} .
$$

Proof. We have $(k \mid \nabla)=(\nabla \mid k)$ since $k$ does not depend on $x \in F$.

Proposition 3.1.6. For all $k \in \mathbb{C}^{d}$, the operator $\Delta_{k}$ formally maps the space of $\Gamma$-periodic functions on $\mathbb{R}^{d}$ into itself.

Proof. This follows because differential operators are local and $\Delta_{k}$ does not depend on $x \in \mathbb{R}^{d}$.

Proposition 3.1.7. For all $k \in \mathbb{C}^{k}, \Delta_{k}$ is formally a normal operator.

Proof. We have $(k \mid \nabla)^{*}=(\bar{k} \mid \nabla)$ and $\left(k^{2}\right)^{*}=\bar{k}^{2}$. The Laplace operator is formally self-adjoint. The operators $\Delta, 4 \pi i(k \mid \nabla),-4 \pi i(\bar{k} \mid \nabla), 4 \pi^{2} k^{2}$ and $4 \pi^{2} \bar{k}^{2}$ mutually commute. The statement is now implied by lemma 3.1 .5 .

Proposition 3.1.6 permits us to formulate the following Schrödinger equation entirely on $F$ :

$$
\left(-\Delta_{k}+u\right) \psi=\lambda \psi \text {. }
$$

We now show that the two approaches are equivalent. 
Lemma 3.1.9. Assume $\psi$ fulfils 3.1 .3 for some $k \in \mathbb{C}^{d}$. Then

$$
x \mapsto e^{-2 \pi i(k \mid x)} \psi(x)
$$

is $\Gamma$-periodic.

Proof. Let $\gamma \in \Gamma$, then

$$
e^{-2 \pi i(k \mid x+\gamma)} \psi(x+\gamma)=e^{-2 \pi i(k \mid x)} e^{-2 \pi i(k \mid \gamma)} e^{2 \pi i(k \mid \gamma)} \psi(x)=e^{-2 \pi i(k \mid x)} \psi(x) .
$$

Theorem 3.1.10. The quantities $k, u, \psi$ and $\lambda$ fulfil the equations (3.1.1), (3.1.2) and (3.1.3) if and only if

$$
\left(-\Delta_{k}+u(x)\right) e^{-2 \pi i(k \mid x)} \psi(x)=\lambda e^{-2 \pi i(k \mid x)} \psi(x) .
$$

Proof. A simple calculation and lemma 3.1.5 show

$$
\Delta e^{2 \pi i(k \mid x)} \psi(x)=e^{2 \pi i(k \mid x)} \Delta_{k} \psi(x) .
$$

Therefore,

$$
\begin{aligned}
(-\Delta+u(x)) \psi(x)=(-\Delta+ & u(x)) e^{2 \pi i(k \mid x)} e^{-2 \pi i(k \mid x)} \psi(x) \\
& =e^{2 \pi i(k \mid x)}\left(-\Delta_{k}+u(x)\right) e^{-2 \pi i(k \mid x)} \psi(x) .
\end{aligned}
$$

Since multiplication with $e^{2 \pi i(k \mid \cdot)}$ is a bijective transformation, the theorem follows.

Due to this theorem, we shall from now on only consider the Schrödinger equation 3.1.8.

\subsection{The resolvent of the free Schrödinger oper- ator}

In this section we investigate the resolvent of the free Schrödinger operator $-\Delta_{0}$ (that is, simply $-\Delta$ ). We shall only be interested in the case $\lambda \in(-\infty,-1]$ as this is not only sufficient for treating the general case in the next section, but also ensures that the resolvent always exists.

We begin with some simple investigations as to what happens if we scale the domains of functions on $F$ by a constant factor.

Definition 3.2.1. Let $f$ be a function defined on $X=F$ or $X=\Gamma^{*}$ and let $c \in \mathbb{C} \backslash\{0\}$. Then we define the scaling operator $\sigma_{c}$ by

$$
\left(\sigma_{c} f\right)(x):=f(c x) .
$$

The scaling operator maps functions on $F$ to functions on $c^{-1} F$ and functions on $\Gamma^{*}$ to functions on $c^{-1} \Gamma^{*}$. 
Lemma 3.2.2. Let $1 \leq q<\infty, m \in \mathbb{Z}_{>0}$ and $c \in \mathbb{C} \backslash\{0\}$. Then $\sigma_{c}$ is a continuous operator $L^{q}(F) \rightarrow L^{q}\left(c^{-1} F\right)$ with norm $|c|^{-d / q}, L^{\infty}(F) \rightarrow L^{\infty}\left(c^{-1} F\right)$ with norm $1, W^{m, q}(F) \rightarrow W^{m, q}\left(c^{-1} F\right)$, with norm $|c|^{m-d / q}$ if $|c| \geq 1$, and $\ell^{q}\left(\Gamma^{*}\right) \rightarrow \ell^{q}\left(c^{-1} \Gamma^{*}\right)$, or, in fact, any rearrangement-invariant Banach function space $E$ on $\Gamma^{*}$ into the corresponding rearrangement-invariant Banach function space $E^{\prime}$ on $c^{-1} \Gamma^{*}$, with norm 1 .

Proof. Let $f \in L^{q}(F)$. The substitution $y=c x$ yields

$$
\left(\int_{c^{-1} F}\left|\left(\sigma_{c} f\right)(x)\right|^{q} \mathrm{~d} x\right)^{1 / q}=\left(\int_{F}|f(y)|^{q}|c|^{-d} \mathrm{~d} y\right)^{1 / q}=|c|^{-d / q}\|f\|_{q}
$$

If $q=\infty$, it is obvious that $\left\|\sigma_{c}\right\|=1$. By a similar calculation, we get for $f \in W^{m, q}(F)$ :

$$
\begin{aligned}
\left\|\sigma_{c} f\right\|_{W^{m, q}}=\sum_{|\alpha| \leq m}\left\|\partial^{\alpha} \sigma_{c} f\right\|_{q} & =\sum_{|\alpha| \leq m}|c|^{|\alpha|}\left\|\sigma_{c} \partial^{\alpha} f\right\|_{q} \\
& =\sum_{|\alpha| \leq m}|c|^{|\alpha|-d / q}\left\|\partial^{\alpha} f\right\|_{q} \leq|c|^{m-d / q}\|f\|_{W^{m, q}}
\end{aligned}
$$

if $|c| \geq 1$. For arbitrary $c$ we can use the upper bound $\max \left\{|c|^{-d / q},|c|^{m-d / q}\right\}$ to prove continuity. The reverse inequality follows by considering a sequence of functions $\left(f_{n}\right)_{n \in \mathbb{N}}$ in $W^{m, q}(F)$ with $\left\|\partial^{\alpha} f_{n}\right\| \rightarrow 0$ for $|\alpha|<m$ and $\left\|\partial^{\alpha} f_{n}\right\|=$ const for $|\alpha|=m$. Finally, since, for $f \in E, f^{\star}$ is just the sequence of absolute values of $f$ in decreasing order, $f^{\star}=\left(\sigma_{c} f\right)^{\star}$. The claim now follows since the norms of $f$ and $\sigma_{c} f$ only depend on their decreasing rearrangement.

Next, let's see what happens when we apply the free resolvent to various spaces.

Theorem 3.2.3. For all $1<q<\infty$ and all $\lambda \in(-\infty,-1]$, the resolvent $\left(\lambda+\Delta_{0}\right)^{-1}$ is defined and maps $L^{q}(F)$ boundedly into $W^{2, q}(F)$. The bound is independent of $\lambda$.

Proof. Since $\lambda$ is negative, we can choose $\sqrt{\lambda}:=i \sqrt{|\lambda|}$. By theorems 2.2.13 and 2.2.14. $\left(1-\Delta_{0}\right)^{-1}$ maps $L^{q}(F)$ into $W^{2, q}(F)$. Now,

$$
\frac{1}{\lambda-4 \pi^{2} \kappa^{2}}=\frac{\lambda^{-1}}{1+4 \pi^{2}(\kappa / \sqrt{\lambda})^{2}},
$$

so the resolvent $\left(\lambda+\Delta_{0}\right)^{-1}$ also maps $L^{q}(F)$ into $W^{2, q}(F)$ by theorem 2.2.13 and lemma 3.2 .2 . It remains to show the independence from $\lambda$. Denote the integral kernel of $\left(\lambda+\Delta_{0}\right)^{-1}$ by $G(\sqrt{\lambda}, \cdot)$. As the proof of theorem 2.2 .13 shows, there corresponds a kernel $G^{\mathbb{R}^{d}}(\sqrt{\lambda}, \cdot)$ to $G(\sqrt{\lambda}, \cdot)$. It follows from GJ87, 7.2 and 7.3] that

$$
G^{\mathbb{R}^{d}}(\sqrt{\lambda}, x)=|\sqrt{\lambda}|^{d-2} \sigma_{\sqrt{\lambda}} G^{\mathbb{R}^{d}}(1, x)
$$


Let $f \in L^{q}(F)$. We now estimate the norm of $\left(\lambda+\Delta_{0}\right)^{-1} f$ :

$$
\begin{aligned}
\left\|\left(\lambda+\Delta_{0}\right)^{-1} f\right\|_{W^{2, q}} & =\left\|\int_{F} G(\sqrt{\lambda}, x-y) f(y) \mathrm{d} y\right\|_{W^{2, q}} \\
& =\left\|\int_{\mathbb{R}^{d}} G^{\mathbb{R}^{d}}(\sqrt{\lambda}, x-y) f(y) \mathrm{d} y\right\|_{W^{2, q}} \\
& =|\sqrt{\lambda}|^{d-2}\left\|\int_{\mathbb{R}^{d}}\left(\sigma_{\sqrt{\lambda}} G^{\mathbb{R}^{d}}(1, x-y)\right) f(y) \mathrm{d} y\right\|_{W^{2, q}} \\
& =|\sqrt{\lambda}|^{d-2}\left\|\sigma_{\sqrt{\lambda}} \int_{\mathbb{R}^{d}} G^{\mathbb{R}^{d}}(1, x-\sqrt{\lambda} y) f(y) \mathrm{d} y\right\|_{W^{2, q}}
\end{aligned}
$$

By lemma 3.2 .2

$$
=|\sqrt{\lambda}|^{d-2}|\sqrt{\lambda}|^{2-d / q}\|\|_{\mathbb{R}^{d}} G^{\mathbb{R}^{d}}(1, x-\sqrt{\lambda} y) f(y) \mathrm{d} y \|_{W^{2, q}}
$$

Substituting $z=\sqrt{\lambda} y$ :

$$
\begin{aligned}
& =|\sqrt{\lambda}|^{d-d / q}\left\|\int_{\mathbb{R}^{d}} G^{\mathbb{R}^{d}}(1, x-z)\left(\sigma_{1 / \sqrt{\lambda}} f\right)(z)|\sqrt{\lambda}|^{-d} \mathrm{~d} z\right\|_{W^{2, q}} \\
& \leq|\sqrt{\lambda}|^{-d / q}\left\|(\mathrm{id}-\Delta)^{-1}\right\|\left\|\sigma_{1 / \sqrt{\lambda}} f\right\|_{q}=\left\|(\mathrm{id}-\Delta)^{-1}\right\|\|f\|_{q} .
\end{aligned}
$$

Hence, $\left\|\left(\lambda+\Delta_{0}\right)^{-1}\right\|$ does not depend on $\lambda$.

The proof of this theorem immediately yields

Corollary 3.2.4. For all $1<q<\infty$, all $1<r<q$ and all $\lambda \in(-\infty,-1]$, the resolvent $\left(\lambda+\Delta_{0}\right)^{-1}$ is defined and maps $L^{q}(F)$ boundedly into $W^{2, r}(F)$. The bound becomes arbitrarily small as $\lambda \rightarrow-\infty$.

This theorem does not extend to the case $q=1$ due to the equivalence failure between Bessel potential and Sobolev spaces. It will turn out that this makes it unusable in the case $d=2$. Instead, we need to apply the free resolvent to different spaces:

Theorem 3.2.5. If $d=2$, then for all $\lambda \in(-\infty,-1]$, the resolvent $\left(\lambda+\Delta_{0}\right)^{-1}$ is defined and maps $\mathcal{F} \ell^{\infty, 1}\left(\Gamma^{*}\right)$ boundedly into $\mathcal{F} \ell^{1}\left(\Gamma^{*}\right)$. The bound is independent of $\lambda$.

Proof. Since we are using Fourier spaces, all we need to show is that the multiplication with

$$
g(\kappa):=\frac{1}{\lambda-4 \pi^{2} \kappa^{2}}
$$

maps $\ell^{\infty, 1}\left(\Gamma^{*}\right)$ boundedly into $\ell^{1}\left(\Gamma^{*}\right)$. For this purpose, we need to estimate the decreasing rearrangement $g^{\star}$. Once again, recall our convention that $g^{\star}(n)$ 
is simply the $n$-th largest element (in terms of absolute value) of the image of $g$. In other words,

$$
g^{\star}(n)=\frac{1}{|\lambda|+4 \pi^{2} \kappa_{n}^{2}},
$$

where $\left(\kappa_{n}\right)_{n \in \mathbb{N}}$ is an arrangement such that $\left\|\kappa_{n}\right\| \geq\left\|\kappa_{n^{\prime}}\right\|$ whenever $n \geq n^{\prime}$. This means that the $\kappa_{1}, \ldots, \kappa_{n}$ lie within concentric balls around the origin whose radius does not decrease with increasing $n$. Since $d=2$, the number of lattice points within each such ball is asymptotically proportional to the square of its radius. This is compensated for by the quadratic nature of $\kappa^{2}$. Hence, there are constants $C_{1}, C_{2}>0$ depending only on $\Gamma^{*}$ such that

$$
\frac{1}{|\lambda|+C_{1}(n-1)} \leq g^{\star}(n) \leq \frac{1}{|\lambda|+C_{2}(n-1)}
$$

We may assume that $C_{2} \leq 1$ (in particular $C_{2} \leq|\lambda|$ ). Let $f \in \ell^{\infty, 1}\left(\Gamma^{*}\right)$. The 1norm of $g f$ can now be estimated easily using the Hardy-Littlewood inequality (theorem 2.3.6):

$$
\begin{aligned}
& \sum_{\kappa \in \Gamma^{*}}|g(\kappa) f(\kappa)| \leq \sum_{n=1}^{\infty} g^{\star}(n) f^{\star}(n) \leq \sum_{n=1}^{\infty} \frac{f^{\star}(n)}{|\lambda|+C_{2}(n-1)} \\
& \leq C_{2}^{-1} \sum_{n=1}^{\infty} \frac{f^{\star}(n)}{n}=C_{2}^{-1}\|f\|_{\infty, 1} .
\end{aligned}
$$

This proves the theorem.

The content of the preceding theorem can be weakened to more familiar functions paces as follows.

Corollary 3.2.9. If $d=2$, then for all $\lambda \in(-\infty,-1]$, the resolvent $\left(\lambda+\Delta_{0}\right)^{-1}$ is defined and maps $L^{1,1 ; 1}(F)$ boundedly into $W^{2,1}(F) \cap \mathcal{F} \ell^{1}\left(\Gamma^{*}\right)$. The bound does not depend on $\lambda$.

Proof. By construction, the Hardy space (see section 2.7.1) is the optimal Banach subspace of $L^{1}(F)$ so that the image of the free resolvent lies in $W^{2,1}(F)$. Now, we have $L^{1,1 ; 1}(F) \subseteq H^{1}(F)$ boundedly by theorem 2.7.2 so the resolvent is bounded from $L^{1,1 ; 1}(F)$ into $W^{2,1}(F)$. To show the independence of the bound from $\lambda$ we prove that the same scaling behaviour as in theorem 3.2 .5 is effective: we already know that $\mathcal{F}$ and $\mathcal{F}^{\prime}$ are of strong type $(1, \infty)$. This shows $\mathcal{F} \ell^{1}\left(\Gamma^{*}\right) \subseteq L^{\infty}(F)$ and, by the left endpoint case of theorem 2.4.12, $L^{1,1 ; 1}(F) \subseteq \mathcal{F} \ell^{\infty, 1}\left(\Gamma^{*}\right)$ boundedly. This last embedding also proves that the free resolvent maps $L^{1,1 ; 1}(F)$ boundedly into $W^{2,1}(F) \cap \mathcal{F} \ell^{1}\left(\Gamma^{*}\right)$ by theorem 3.2.5. Finally, $W^{2,1}(F) \subseteq C^{0}(F) \subseteq L^{\infty}(F)$ by Sobolev's theorem 2.2.2 All these embeddings are sharp with respect to their scaling behaviour (lemma 3.2.2).

Since we do not have a Rellich-Kondrashov theorem for our Fourier spaces, we need to prove the following compactness results "by hand".

Theorem 3.2.10. If $d=2$, then for all $1 \leq p, q \leq \infty$ with $1 / q-1 / p<1$ and all $\lambda \in(-\infty,-1]$, the resolvent $\left(\lambda+\Delta_{0}\right)^{-1}$ is defined and maps $\mathcal{F} \ell^{p, 1}\left(\Gamma^{*}\right)$ compactly into $\mathcal{F} \ell^{q, 1}\left(\Gamma^{*}\right)$. The bound vanishes as $\lambda \rightarrow-\infty$. 
Proof. In the given cases, the resolvent is continuous due to theorem 3.2.5, with vanishing bounds for $\lambda \rightarrow-\infty$ due to scale reasons. It remains to show that the resolvent is compact in all cases.

Let $\left(P_{m}\right)_{m \in \mathbb{N}}$ be a sequence of finite-rank projections of functions defined on $\Gamma^{*}$ such that for each $m \in \mathbb{N}, P_{m}$ projects onto the space generated by the Fourier modes belonging to the $m$ basis vectors $\kappa$ with lowest norm. It then follows from 3.2 .8 that

$$
\frac{1}{|\lambda|+C_{1}(n+m-1)} \leq\left(\left(1-P_{m}\right) g\right)^{\star}(n) \leq \frac{1}{|\lambda|+C_{2}(n+m-1)} .
$$

with the same constants $C_{1}, C_{2}$ for all $m \in \mathbb{N}$. Now let $f \in \ell^{p, 1}\left(\Gamma^{*}\right)$, then once again by the Hardy-Littlewood inequality (corollary 2.3.8) and, when $n \mapsto$ $n^{1 / q-1}$ is decreasing, by Hardy's lemma (proposition 2.3.5):

$$
\begin{aligned}
\left\|g f-P_{m} g f\right\|_{q, 1} \leq \sum_{n=1}^{\infty}\left(\left(1-P_{m}\right) g\right)^{\star}(n) f^{\star}(n) n^{1 / q-1} & \\
& \leq \sup _{n \in \mathbb{N}} \frac{n^{1 / q-1 / p}}{|\lambda|+C_{2}(n+m-1)}\|f\|_{p, 1} .
\end{aligned}
$$

The supremum vanishes for $m \rightarrow \infty$ whenever $1 / q-1 / p<1$. Hence the free Schrödinger resolvent is, in this case, the limit of a sequence of finite-rank operators and therefore compact by proposition 2.2 .4 .

For technical reasons, we shall need the following "halfway" result when adding boundary conditions in the $d=2$ case later on.

Proposition 3.2.12. If $d=2$, then for all $k \in \mathbb{C}^{2}$ and all $\lambda \in(-\infty,-1]$ the operator

$$
(k \mid \nabla)\left(\lambda+\Delta_{0}\right)^{-1}
$$

is defined and maps $\mathcal{F} \ell^{\infty, 1}\left(\Gamma^{*}\right)$ boundedly into $\mathcal{F} \ell^{2,1}\left(\Gamma^{*}\right)$. The bound does not depend on $\lambda$. Moreover, for all $1 \leq p, q \leq \infty$ with $1 / q-1 / p<1 / 2$, the operator 3.2 .13 is defined and maps $\mathcal{F} \ell^{p, 1}\left(\Gamma^{*}\right)$ compactly into $\mathcal{F} \ell^{q, 1}\left(\Gamma^{*}\right)$. In this case, the bound vanishes as $\lambda \rightarrow-\infty$.

Proof. The proof works along similar lines as the proof of theorem 3.2.5. This time, we first need to show that the multiplication with

$$
g(\kappa):=\frac{2 \pi i(k \mid \kappa)}{\lambda-4 \pi^{2} \kappa^{2}}
$$

maps $\ell^{\infty, 1}\left(\Gamma^{*}\right)$ boundedly into $\ell^{2,1}\left(\Gamma^{*}\right)$. In analogy to 3.2 .7 , we have

$$
g^{\star}(n)=\frac{2 \pi|k|\left|\kappa_{n}\right|}{|\lambda|+4 \pi^{2} \kappa_{n}^{2}},
$$

where $\left(\kappa_{n}\right)_{n \in \mathbb{N}}$ is an arrangement such that $\left|\kappa_{n}\right| \geq\left|\kappa_{n^{\prime}}\right|$ whenever $n \geq n^{\prime}$. With similar reasoning with which we arrived at (3.2.8), we have

$$
\frac{C_{1} \sqrt{n-1}}{|\lambda|+C_{2}(n-1)} \leq g^{\star}(n) \leq \frac{C_{3} \sqrt{n-1}}{|\lambda|+C_{4}(n-1)},
$$


where $C_{1}, C_{2}, C_{3}, C_{4}>0, C_{4} \leq 1$. Let $f \in \ell^{\infty, 1}\left(\Gamma^{*}\right)$. The $\ell^{2,1}$-norm of $g f$ can again be estimated with the Hardy-Littlewood inequality, this time in combination with Hardy's lemma (proposition 2.3.5 in combination with corollary 2.3.8):

$$
\sum_{n=1}^{\infty} \frac{(g f)^{\star}(n)}{\sqrt{n}} \leq \sum_{n=1}^{\infty} \frac{1}{\sqrt{n}} \frac{f^{\star}(n) C_{3} \sqrt{n-1}}{|\lambda|+C_{4}(n-1)} \leq \frac{C_{3}}{C_{4}} \sum_{n=1}^{\infty} \frac{f^{\star}(n)}{n}=\frac{C_{3}}{C_{4}}\|f\|_{\infty, 1} .
$$

This shows the first part of the proposition. It is also clear that for the second part, this operator is continuous as well, with vanishing bound for $\lambda \rightarrow-\infty$ due to scale reasons. It remains to prove the compactness claim. Thereto we take the sequence of finite-rank projectors $\left(P_{m}\right)_{m \in \mathbb{N}}$ from the proof of theorem 3.2 .10 to gain from 3.2.14 the estimate

$$
\frac{C_{1} \sqrt{n+m-1}}{|\lambda|+C_{2}(n+m-1)} \leq\left(\left(1-P_{m}\right) g\right)^{\star}(n) \leq \frac{C_{3} \sqrt{n+m-1}}{|\lambda|+C_{4}(n+m-1)} .
$$

For $f \in \ell^{p, 1}\left(\Gamma^{*}\right)$ we now have the following estimate similar to (3.2.11):

$$
\begin{aligned}
\left\|g f-P_{m} g f\right\|_{q, 1} \leq \sum_{n=1}^{\infty}\left(\left(1-P_{m}\right) g\right)^{\star}(n) f^{\star}(n) n^{1 / q-1} & \\
& \leq \sup _{n \in \mathbb{N}} \frac{C_{3} \sqrt{n+m-1}}{|\lambda|+C_{4}(n+m-1)} n^{1 / q-1 / p}\|f\|_{p, 1} .
\end{aligned}
$$

This time, the supremum vanishes for $m \rightarrow \infty$ whenever $1 / q-1 / p<1 / 2$, proving the claim.

Note that the combination of the first-order differential operator $(k \mid \nabla)$ with the second-order resolvent $\left(\lambda+\Delta_{0}\right)^{-1}$ is crucial here. For example, multiplication of a generic $\ell^{1}$-function with $(k \mid \kappa)$ does not necessarily yield an $\ell^{2,1}$-function.

We have thus established the free resolvent of the Schrödinger operator as a continuous or even compact operator on various spaces, for certain $\lambda$, but without boundary conditions. Although we won't immediately need boundary conditions in the next section, we remark here that it is possible to include boundary conditions at this stage. We have

Lemma 3.2.15. Let $V \subseteq \mathbb{C}^{d}$ be bounded. Then

$$
\inf _{\substack{k \in V \\ \kappa \in \Gamma^{*}}} \Re(\kappa+k)^{2}>-\infty .
$$

Proof. Set

$$
S:=\sup _{k \in V}|k| .
$$

Since $V$ is bounded, $S<\infty$. Since $\Gamma^{*} \subseteq \mathbb{R}^{d}$, we now have

$$
\begin{aligned}
\Re(\kappa+k)^{2}=\sum_{i=1}^{d}\left(\kappa_{i}^{2}+2 \kappa_{i} \Re k_{i}+\left(\Re k_{i}\right)^{2}-\left(\Im k_{i}\right)^{2}\right) \\
\quad \geq \sum_{i=1}^{d}\left(\left|\kappa_{i}\right|\left(\left|\kappa_{i}\right|-2 S\right)-S^{2}\right) \geq \sum_{i=1}^{d}\left(-4 S^{2}-S^{2}\right)=-5 d S^{2} .
\end{aligned}
$$


Therefore, the previous results remain true with boundary conditions from a bounded set $V \subseteq \mathbb{C}^{d}$ with bound $S$ if one takes $\lambda \in(-\infty, B]$ instead of $\lambda \in$ $(-\infty,-1]$, where, say, $B=-5 d S^{2}-1$ (thus obviously $B$ depends on $V$ ), and, where applicable, replaces the independence of the resolvent norm of $\lambda$ with uniform boundability with respect to $k \in V$.

\subsection{Resolvents of general Schrödinger operators}

Now that we have established the behaviour of the free resolvent in the previous section, we shall investigate the resolvent step by step for arbitrary potentials $u$, arbitrary boundary conditions $k$ and, eventually, arbitrary $\lambda$.

First, we need a simple restatement of the triangle inequality.

Lemma 3.3.1. Let $E$ be any normed space and let $\delta>\epsilon>0$. Let $x, y, z \in E$ such that $\|z-x\| \geq \delta$. Then $\|y-x\| \geq \epsilon$ or $\|z-y\| \geq \delta-\epsilon$.

Proof. Assume $\|y-x\|<\epsilon$. By the triangle inequality

$$
\|z-x\| \leq\|z-y\|+\|y-x\|
$$

so

$$
\|z-y\| \geq\|z-x\|-\|y-x\| \geq \delta-\epsilon .
$$

Next, we introduce some important open subsets of our function spaces. Recall the definitions of the $r$-localised norms and the $N$-localised quasi-norms from section 2.8 ,

Definition 3.3.2. Let $E$ be an absolutely continuous quasi-Banach function space and $C, r>0$. Then we denote the open ball around the origin of radius $C$ with respect to the $r$-localised norm by $B_{C}^{r}$. Likewise, if $E=\ell^{\infty, 1}\left(\Gamma^{*}\right)$ and $N \in$ $\mathbb{N}$, we denote the open ball around the origin of radius $C$ with respect to the $N$-localised quasi-norm by $B_{C}^{N}$. These definitions extend to Fourier spaces in the obvious way.

Proposition 3.3.3. The sets $B_{C}^{r}$ and $B_{C}^{N}$ are invariant under translation operators as introduced in definition 2.8.20.

Proof. The invariance of $B_{C}^{r}$ follows immediately from the definition of the $r$ localised norm. The invariance of $B_{C}^{N}$ follows because the $N$-localised quasinorm depends only on the decreasing rearrangement.

Now, we shall investigate the operator $u\left(\lambda+\Delta_{0}\right)^{-1}$.

Lemma 3.3.4. Let $d>2$. Then for all $1<q<d / 2$ and all $u_{0} \in L^{d / 2}(F)$ there are $C, r>0$ with $u_{0} \in B_{C}^{r}$ and $a \lambda_{0} \in(-\infty,-1]$ such that the operator $u(\lambda+$ $\left.\Delta_{0}\right)^{-1}$ is bounded on $L^{q}(F)$ for all $u \in B_{C}^{r}$ and all $\lambda \leq \lambda_{0}$ with a bound smaller than one with respect to the $r$-localised norm on $L^{q}(F)$.

Proof. By theorem 3.2.3 and Sobolev's theorem 2.2.3 $\left(\lambda+\Delta_{0}\right)^{-1}$ maps $L^{q}(F)$ boundedly into $L^{(1 / q-2 / d)^{-1}}(F)$, so by Hölder's inequality, theorem 2.1.1. $u(\lambda+$ $\left.\Delta_{0}\right)^{-1}$ maps $L^{q}(F)$ boundedly into itself for all $u \in L^{d / 2}(F)$ and all $\lambda \in$ 
$(-\infty,-1]$. The interesting part is in which cases the norm of this operator is smaller than one.

Let $G(\sqrt{\lambda}, \cdot)$ be the integral kernel of $\left(\lambda+\Delta_{0}\right)^{-1}$ and $G^{\mathbb{R}^{d}}(\sqrt{\lambda}, \cdot)$ the corresponding kernel for $\mathbb{R}^{d}$. Recall our notation conventions for characteristic functions of balls from definition 2.8.10 and set $G^{r}(\sqrt{\lambda}, x):=G^{\mathbb{R}^{d}}(\sqrt{\lambda}, x) \chi_{r}^{0}(x)$, and $H^{r}(\sqrt{\lambda}, x):=G^{\mathbb{R}^{d}}(\sqrt{\lambda}, x)\left(1-\chi_{r}^{0}(x)\right)$, for all $r>0$. According to proposition 2.8.7. we can choose $r>0$ sufficiently small, such that we have for the $r$-localised norm

$$
\left\|u_{0}\right\|_{d / 2 ; r}<\frac{1}{\nu_{r, r / 2}\left\|\left(\lambda-\Delta_{0}\right)^{-1}\right\|}=: C
$$

Recall that $\nu_{r, r / 2}$ is the minimum number of balls of radius $r / 2$ required to cover a ball of radius $r$. By lemma 2.8.9, this number does not depend on $r$. Furthermore, by theorem 3.2.3, $r$ and $C$ do not depend on $\lambda$. Clearly, (3.3.5) is fulfilled for all $u \in B_{C}^{r}$. Since the kernel $G^{\mathbb{R}^{d}}(\sqrt{\lambda}, \cdot)$ decays exponentially with decay constant $|\sqrt{\lambda}|$ near infinity [GJ87, 7.2], the norms of the operators

$$
f \mapsto u\left(H^{r / 2} * f\right)
$$

can be diminished arbitrarily by making $|\lambda|$ very large. To estimate the norm of the operators

$$
f \mapsto u\left(G^{r / 2} * f\right),
$$

we use lemma 3.3 .1 to gain the inequality

$$
\begin{aligned}
\left|u(y) \int_{\mathbb{R}^{d}} G^{\mathbb{R}^{d}}(\sqrt{\lambda}, y-z) \chi_{r / 2}^{0}(y-z) f(z) \mathrm{d} z \chi_{r / 2}^{x}(y)\right| & \\
& \leq\left|u(y) \chi_{r / 2}^{x}(y) \int_{\mathbb{R}^{d}} G^{\mathbb{R}^{d}}(\sqrt{\lambda}, y-z) \chi_{r / 2}^{0}(y-z) \chi_{r}^{x}(z) f(z) \mathrm{d} z\right|
\end{aligned}
$$

for all $x, y$. Since $\left(\lambda-4 \pi^{2} \kappa^{2}\right)^{-1}$ is strictly negative, the norm of the operator induced by the $G^{r / 2}$ kernel is smaller than $\left\|\left(\lambda-\Delta_{0}\right)^{-1}\right\|$. Therefore, we have shown that $u\left(\lambda-\Delta_{0}\right)^{-1}$ (with large $|\lambda|$ ) has, by (3.3.5), operator norm smaller than $\nu_{r, r / 2}^{-1}$ when considered as an operator from $L^{q}(F)$ with $r$-localised norm to $L^{q}(F)$ with $(r / 2)$-localised norm. A final appeal to theorem 2.8.11 then shows that the operator norm is smaller than one when using the $r$-localised norm on $L^{q}(F)$ exclusively.

As in the previous section, this lemma does not extend to the case $d=2$. Instead, we prove the following

Lemma 3.3.6. Let $d=2$. Then for all $u_{0} \in \mathcal{F} \ell^{\infty, 1}\left(\Gamma^{*}\right)$ there is an $N \in$ $\mathbb{N}$ and $a C>0$ with $u_{0} \in B_{C}^{N}$ as well as a $\lambda_{0} \in(-\infty,-1]$ such that the operator $u\left(\lambda+\Delta_{0}\right)^{-1}$ is bounded on $\mathcal{F} \ell^{\infty, 1}\left(\Gamma^{*}\right)$ for all $u \in B_{C}^{N}$ and all $\lambda \leq \lambda_{0}$ with bound smaller than one.

Proof. Proposition 2.4 .15 and theorem 3.2 .5 imply that

$$
\left\|u\left(\lambda+\Delta_{0}\right)^{-1} f\right\|_{\mathcal{F} \infty, 1} \leq\|u\|_{\mathcal{F} \infty, 1}\left\|\left(\lambda+\Delta_{0}\right)^{-1}\right\|\|f\|_{\mathcal{F} \infty, 1}
$$


for all $u, f \in \mathcal{F} \ell^{\infty, 1}\left(\Gamma^{*}\right)$ and all $\lambda \in(-\infty,-1]$. So once more, we "only" must make the operator norm smaller than one.

By proposition 2.8.18, there is an $N \in \mathbb{N}$ such that

$$
\left\|\widehat{u}_{0}\right\|_{(\infty, 1) ; N}<\frac{\mu(F)}{2\left\|\left(\lambda+\Delta_{0}\right)^{-1}\right\|}=: C .
$$

By theorem 3.2.5 $N$ and $C$ do not depend on $\lambda$. Clearly, 3.3.7 holds for all $u \in B_{C}^{N}$. Now, for each such $u$ there exists an $A_{u} \subseteq \Gamma^{*}$ with $\mu\left(A_{u}\right)=N$ such that the $N$-localised quasi-norm of $u$ equals the $A_{u}$-localised norm. Since $B_{C}^{N}$ is bounded, it follows that

$$
c:=\sup _{u \in B_{C}^{N}}\left\|\widehat{u}-\widehat{u}_{A_{u}}\right\|_{\infty}<\infty .
$$

Let $\widehat{G}_{\lambda}(\kappa):=\left(\lambda-4 \pi^{2} \kappa^{2}\right)^{-1}$. We decompose each $u \in B_{C}^{N}$ by

$$
\widehat{u}_{1}:=\widehat{u}_{A_{u}}, \quad \widehat{u}_{2}:=\widehat{u}-\widehat{u}_{A_{u}} .
$$

The monotonicity property and (3.3.7) imply that

$$
\frac{1}{\mu(F)}\left\|\widehat{u}_{1} *\left(\widehat{G}_{\lambda} \widehat{f}\right)\right\|_{\infty, 1}<\frac{1}{2}\|\widehat{f}\|_{\infty, 1}
$$

for all nonzero $\widehat{f} \in \ell^{\infty, 1}\left(\Gamma^{*}\right)$. We now make the analogous estimate for $\widehat{u}_{2}$. We have the estimate

$$
\left|\left(\widehat{u}_{2} *\left(\widehat{G}_{\lambda} \widehat{f}\right)\right)(\kappa)\right|=\left|\sum_{\nu \in \Gamma^{*}} \widehat{u}_{2}(\kappa-\nu) \widehat{G}_{\lambda}(\nu) \widehat{f}(\nu)\right| \leq \sum_{\kappa-\nu \in A_{u}} c\left|\widehat{G}_{\lambda}(\nu) \widehat{f}(\nu)\right| .
$$

By proposition 2.3.4, this yields

$$
\begin{aligned}
\left\|\widehat{u}_{2} *\left(\widehat{G}_{\lambda} \widehat{f}\right)\right\|_{\infty, 1} & \leq c \sum_{n=1}^{\infty} \frac{1}{n}\left(\kappa \mapsto \sum_{\kappa-\nu \in A_{u}}\left|\frac{\widehat{f}(\nu)}{\lambda-4 \pi^{2} \nu^{2}}\right|\right)^{\star}(n) \\
& \leq \frac{c}{|\lambda|} \sum_{n=1}^{\infty} \frac{1}{n}\left(\kappa \mapsto \sum_{\kappa-\nu \in A_{u}}|\widehat{f}(\nu)|\right)^{\star}(n)
\end{aligned}
$$

Now, since $\mu\left(A_{u}\right)=N$ for all $u \in B_{C}^{N}$ we have for a suitable levelling operator $\Lambda$ (depending on $A_{u}$ ):

$$
\leq \frac{c}{|\lambda|} \sum_{n=1}^{\infty} \frac{1}{n}(N \Lambda|\widehat{f}|)^{\star}(n)=\frac{c N}{|\lambda|}\|\Lambda|\widehat{f}|\|_{\infty, 1}
$$

By proposition 2.8 .23 and the equiabsoluteness property:

$$
\leq \frac{c N}{|\lambda|}\|\widehat{f}\|_{\infty, 1}
$$

Hence, $\left\|\widehat{u}_{2} *\left(\widehat{G}_{\lambda} \widehat{f}\right)\right\|_{\infty, 1}$ can be diminished arbitrarily by making $|\lambda|$ very large. The lemma now follows due to a suitable extension of the convolution theorem 2.1.10. 
Note that in the proof of this lemma, we did not use localised norm as explicitly as in lemma 3.3.4. Rather, once 3.3.7 was established, we could withdraw from the $N$-localised norm to the usual norm on $\ell^{\infty, 1}\left(\Gamma^{*}\right)$ via $A_{u}$-localised norms and then exploit the monotonicity property. Still, the $N$-localised norm will continue to accompany us in the guise of the sets $B_{C}^{N}$.

A weakened version of this lemma using more familiar spaces would be the following

Lemma 3.3.11. Let $d=2$. Then for all $u_{0} \in L^{1,1 ; 1}(F)$ there are $C, r>0$ with $u_{0} \in B_{C}^{r}$ and a $\lambda_{0} \in(-\infty,-1]$ such that the operator $u\left(\lambda+\Delta_{0}\right)^{-1}$ is bounded on $L^{1,1 ; 1}(F)$ for all $u \in B_{C}^{r}$ and all $\lambda \leq \lambda_{0}$ with a bound smaller than one with respect to the $r$-localised norm on $L^{1,1 ; 1}(F)$.

Proof. By Sobolev's theorem 2.2.2 we have $W^{2,1}(F) \subseteq C^{0}(F) \subseteq L^{\infty}(F)$. Therefore, it follows from corollary 3.2 .9 and Hölder's inequality (theorem 2.4.6) that $u\left(\lambda+\Delta_{0}\right)^{-1}$ is a bounded operator on $L^{1,1 ; 1}(F)$ for all $u \in L^{1,1 ; 1}(F)$ and all $\lambda \in(-\infty,-1]$. The equivalent renorming now proceeds as in the proof of lemma 3.3 .4

Due to lemma 3.2.15 and the remarks thereafter, it is possible to prove the previous lemmata with boundary conditions from a bounded set. Yet, we define the resolvent still without boundary condition for all potentials and negative $\lambda$ with sufficiently large absolute value (depending on the potential), and add boundary conditions later.

Proposition 3.3.12. Let $d>2$. Then for all $1<q<d / 2$ and all $u_{0} \in$ $L^{d / 2}(F)$ there are $C, r>0$ with $u_{0} \in B_{C}^{r}$ and $a \lambda_{0} \in(-\infty,-1]$ such that the resolvent $\left(\lambda+\Delta_{0}-u\right)^{-1}$ is defined and maps $L^{q}(F)$ boundedly into $W^{2, q}(F)$ for all $u \in B_{C}^{r}$ and all $\lambda \leq \lambda_{0}$.

Proof. By lemma 3.3.4 there are $C, r>0$ with $u_{0} \in B_{C}^{r}$ and a $\lambda_{0} \in(-\infty,-1]$ such that $u\left(\lambda+\Delta_{0}\right)^{-1}$ maps $L^{q}(F)$ into itself for all $u \in B_{C}^{r}$ and all $\lambda \leq \lambda_{0}$, and the operator norm can be made smaller than one by equivalent renorming. Hence, the operator $1-u\left(\lambda+\Delta_{0}\right)^{-1}$ is boundedly invertible by the Neumann theorem. Furthermore, by theorem 3.2 .3 , the operator $\left(\lambda+\Delta_{0}\right)^{-1}$ maps $L^{q}(F)$ boundedly into $W^{2, q}(F)$. Now,

$$
\begin{aligned}
\left(\lambda+\Delta_{0}\right)^{-1}(1 & \left.-u\left(\lambda+\Delta_{0}\right)^{-1}\right)^{-1} \\
& =\left(\left(1-u\left(\lambda+\Delta_{0}\right)^{-1}\right)\left(\lambda+\Delta_{0}\right)\right)^{-1}=\left(\lambda+\Delta_{0}-u\right)^{-1}
\end{aligned}
$$

concluding the proof.

Corollary 3.3.14. Let $d>2$. Then for all $1<q<d / 2$, all $1<s<q$ and all $u_{0} \in L^{d / 2}(F)$ the resolvent $\left(\lambda+\Delta_{0}-u\right)^{-1}$ is defined and maps $L^{q}(F)$ boundedly into $W^{2, s}(F)$ for all $u \in B_{C}^{r}$ and all $\lambda \leq \lambda_{0}$, with the same $C, r, \lambda_{0}$ as in proposition 3.3.12. The operator norm vanishes as $\lambda \rightarrow-\infty$.

Proof. This follows from corollary 3.2.4 and the construction 3.3.13.

Proposition 3.3.15. Let $d=2$. Then for all $u_{0} \in \mathcal{F} \ell^{\infty, 1}\left(\Gamma^{*}\right)$ the resolvent $(\lambda+$ $\left.\Delta_{0}-u\right)^{-1}$ is defined and maps $\mathcal{F} \ell^{\infty, 1}\left(\Gamma^{*}\right)$ boundedly into $\mathcal{F} \ell^{1}\left(\Gamma^{*}\right)$ for all $u \in B_{C}^{N}$ and all $\lambda \leq \lambda_{0}$, with the same $N, C, \lambda_{0}$ as in lemma 3.3.6. 
Proof. This follows from lemma 3.3.6 and theorem 3.2 .5 in the same manner as in the proof of proposition 3.3 .12

Again, we have in analogy to corollary 3.2 .9

Corollary 3.3.16. Let $d=2$. Then for all $u_{0} \in L^{1,1 ; 1}(F)$ there are $C, r>0$ with $u_{0} \in B_{C}^{r} \lambda_{0} \in(-\infty,-1]$ such that the resolvent $\left(\lambda+\Delta_{0}-u\right)^{-1}$ is defined and maps $L^{1,1 ; 1}(F)$ boundedly into $W^{2,1}(F) \cap \mathcal{F} \ell^{1}\left(\Gamma^{*}\right)$ for all $u \in B_{C}^{r}$ and all $\lambda \leq \lambda_{0}$.

Proof. This follows from lemma 3.3 .11 and corollary 3.2 .9 by constructing the resolvent as in (3.3.13) using the Neumann theorem.

Let us now generalise theorem 3.2 .10

Proposition 3.3.17. If $d=2$, then for all $1 \leq p, q \leq \infty$ with $1 / q-1 / p<1$ and all $u_{0} \in \mathcal{F} \ell^{\infty, 1}\left(\Gamma^{*}\right)$ the resolvent $\left(\lambda+\Delta_{0}-u\right)^{-1}$ is defined for all $u \in B_{C}^{N}$ and all $\lambda \leq \lambda_{0}$, with the same $N, C, \lambda_{0}$ as in lemma 3.3.6 and maps $\mathcal{F} \ell^{p, 1}\left(\Gamma^{*}\right)$ compactly into $\mathcal{F} \ell^{q, 1}\left(\Gamma^{*}\right)$. The bound vanishes as $\lambda \rightarrow-\infty$.

Proof. Since equation (3.3.13) is due to a Neumann representation, we conclude that

$$
\begin{aligned}
\left(\lambda+\Delta_{0}-u\right)^{-1} & =\left(\lambda+\Delta_{0}\right)^{-1}\left(1-u\left(\lambda+\Delta_{0}\right)^{-1}\right)^{-1} \\
& =\left(1-\left(\lambda+\Delta_{0}\right)^{-1} u\right)^{-1}\left(\lambda+\Delta_{0}\right)^{-1} .
\end{aligned}
$$

We can now use lemma 3.3.6 again to construct the sought operator using theorem 3.2 .10 for the $\left(\lambda+\Delta_{0}\right)^{-1}$-factor. The bound then vanishes as $\lambda \rightarrow-\infty$. Compactness follows due to proposition 2.2.4.

We shall also need the following generalisation of proposition 3.2.12.

Proposition 3.3.19. Let $d=2$. Then for all $u_{0} \in \mathcal{F} \ell^{\infty, 1}\left(\Gamma^{*}\right)$ the operator

$$
(k \mid \nabla)\left(\lambda+\Delta_{0}-u\right)^{-1}
$$

is defined and maps $\mathcal{F} \ell^{\infty, 1}\left(\Gamma^{*}\right)$ boundedly into $\mathcal{F} \ell^{2,1}\left(\Gamma^{*}\right)$ for all $k \in \mathbb{C}^{2}$, all $u \in$ $B_{C}^{N}$ and all $\lambda \leq \lambda_{0}$, with the same $N, C, \lambda_{0}$ as in lemma 3.3.6. Moreover, for all $1 \leq p, q \leq \infty$ with $1 / q-1 / p<1 / 2$, the operator (3.3.20) is defined and maps $\mathcal{F} \ell^{p, 1}\left(\Gamma^{*}\right)$ compactly into $\mathcal{F} \ell^{q, 1}\left(\Gamma^{*}\right)$. In this case, the bound vanishes as $\lambda \rightarrow-\infty$.

Proof. By proposition 3.3.15, there are appropriate $N, C$ and $\lambda_{0}$ such that the operator $\left(\lambda+\Delta_{0}-u\right)^{-1}$ is defined and maps $\mathcal{F} \ell^{\infty, 1}\left(\Gamma^{*}\right)$ boundedly into $\mathcal{F} \ell^{1}\left(\Gamma^{*}\right)$ for all $u \in B_{C}^{N}$ and all $\lambda \leq \lambda_{0}$. This operator can be built through the construction (3.3.13). Multiplying this equation with $(k \mid \nabla)$ from the left, the claim now follows from proposition 3.2.12, with the compactness of the entire operator following from proposition 2.2 .4 .

Now we finally add boundary conditions.

Proposition 3.3.21. Let $d>2$. Then for all $1<q<d / 2$ and all $u_{0} \in L^{d / 2}(F)$ there are $C, r>0$ with $u_{0} \in B_{C}^{r}$ such that for all bounded $V \subseteq \mathbb{C}^{d}$ there is a $\lambda_{0} \in(-\infty,-1]$ such that the resolvent $\left(\lambda+\Delta_{k}-u\right)^{-1}$ is defined and 
maps $L^{q}(F)$ boundedly into $W^{2, q}$, and also $L^{2}(F)$ compactly into itself, for all $k \in V, u \in B_{C}^{r}$ and all $\lambda \leq \lambda_{0}$. Moreover, the map

$$
\begin{aligned}
B_{C}^{r} \times V & \rightarrow \mathcal{K}\left(L^{2}(F), L^{2}(F)\right), \\
(u, k) & \mapsto\left(\lambda+\Delta_{k}-u\right)^{-1}
\end{aligned}
$$

is continuous with respect to the weak topology on $B_{C}^{r}$ and the strong topologies on $V$ and $\mathcal{K}\left(L^{2}(F), L^{2}(F)\right)$, for all $\lambda \leq \lambda_{0}$. Continuity is uniform with respect to $k$.

Proof. Let $\left(q^{-1}+d^{-1}\right)^{-1}<s<q$ such that $s>1$. The operator $\Delta_{k}-\Delta_{0}=$ $4 \pi i(k \mid \nabla)-4 \pi^{2} k^{2}$ maps $W^{2, s}(F)$ boundedly into $W^{1, s}$ because $F$ is compact. Since $V$ is bounded, a uniform upper bound for the norm of the $\Delta_{k}-\Delta_{0}$ can be found. Now, due to the Rellich-Kondrashov theorem 2.2.10, $W^{1, s}(F)$ embeds continuously into $L^{q}(F)$. Due to corollary 3.3.14 there are $C, r>0$ with $u_{0} \in B_{C}^{r}$ and a $\lambda_{0} \in(-\infty,-1]$ of sufficiently large absolute value such that $\left(\lambda+\Delta_{0}-u\right)^{-1}$ maps $L^{q}(F)$ boundedly into $W^{2, s}(F)$ with such a bound that

$$
\left\|\left(\Delta_{k}-\Delta_{0}\right)\left(\lambda+\Delta_{0}-u\right)^{-1}\right\|<1
$$

for all $u \in B_{C}^{r}$ and all $\lambda \leq \lambda_{0}$. Therefore, the operator

$$
1+\left(\Delta_{k}-\Delta_{0}\right)\left(\lambda+\Delta_{0}-u\right)^{-1}
$$

is boundedly invertible as an operator on $L^{q}(F)$ by the Neumann theorem. Hence, we have

$$
\begin{aligned}
\left(\lambda+\Delta_{0}-u\right)^{-1}(1+ & \left.\left(\Delta_{k}-\Delta_{0}\right)\left(\lambda+\Delta_{0}-u\right)^{-1}\right)^{-1} \\
=((1+ & \left.\left.\left(\Delta_{k}-\Delta_{0}\right)\left(\lambda+\Delta_{0}-u\right)^{-1}\right)\left(\lambda+\Delta_{0}-u\right)\right)^{-1} \\
& =\left(\lambda+\Delta_{0}-u+\Delta_{k}-\Delta_{0}\right)^{-1}=\left(\lambda+\Delta_{k}-u\right)^{-1}
\end{aligned}
$$

by proposition 3.3 .12 a bounded operator from $L^{q}(F)$ to $W^{2, q}(F)$ for all $u \in$ $B_{C}^{r}$, all $k \in V$, and all sufficiently small $\lambda$. Choosing $q<2$, we can have this operator act on $L^{2}(F)$ by proposition 2.1.3. On the other hand, choosing $q>(1 / 2+2 / d)^{-1}$ makes $L^{2}(F)$ a valid target space for this operator by the Rellich-Kondrashov theorem 2.2.10. Since this embedding is compact, the entire operator is compact by proposition 2.2 .4

The Hilbert space $L^{2}(F)$, and also the other spaces in question have a Schauder basis, so this compact operator can be approximated by a sequence of finite-rank operators. We shall develop one such approximation now. Each Schauder basis induces a sequence $\left(P_{l}\right)_{l \in \mathbb{N}}$ of finite-rank projectors with increasing images exhausting a dense, countable subset (cf. the proof of theorem 3.2.10). We shall let these operators act on different spaces without changing notation, as a common subset which is dense in all these spaces can be found. Now, the sequence $\left(P_{l} u\right)_{l \in \mathbb{N}}$ on $L^{d / 2}(F)$ converges weakly to $u$. We can use this fact to build an approximation using the Neumann representation of the resolvent. By 
construction,

$$
\begin{aligned}
\left(\lambda+\Delta_{k}-u\right)^{-1}= & \left(\lambda+\Delta_{0}-u\right)^{-1}\left(1+\left(\Delta_{k}-\Delta_{0}\right)\left(\lambda+\Delta_{0}-u\right)^{-1}\right)^{-1} \\
= & \left(\lambda+\Delta_{0}\right)^{-1}\left(1-u\left(\lambda+\Delta_{0}\right)^{-1}\right)^{-1} \\
& \cdot\left(1+\left(\Delta_{k}-\Delta_{0}\right)\left(\lambda+\Delta_{0}\right)^{-1}\left(1-u\left(\lambda+\Delta_{0}\right)^{-1}\right)^{-1}\right)^{-1} \\
= & \left(\lambda+\Delta_{0}\right)^{-1} \sum_{n=0}^{\infty}\left(u\left(\lambda+\Delta_{0}\right)^{-1}\right)^{n} \\
& \cdot \sum_{m=0}^{\infty}(-1)^{m}\left(\left(\Delta_{k}-\Delta_{0}\right)\left(\lambda+\Delta_{0}\right)^{-1} \sum_{n=0}^{\infty}\left(u\left(\lambda+\Delta_{0}\right)^{-1}\right)^{n}\right)^{m} .
\end{aligned}
$$

Note that due to lemma 3.2 .15 , it is possible to simplify this representation (possibly with a different $\lambda$ ) to

$$
\left(\lambda+\Delta_{k}-u\right)^{-1}=\left(\lambda+\Delta_{k}\right)^{-1} \sum_{n=0}^{\infty}\left(u\left(\lambda+\Delta_{k}\right)^{-1}\right)^{n} .
$$

We shall only use this simpler representation for an exemplary derivation of the sought approximation. Since $\left(\lambda+\Delta_{k}\right)^{-1}$ is compact as an operator from $L^{q}(F)$ $\left(L^{d / 2}(F)\right.$ in particular) to $L^{2}(F),\left(\left(\lambda+\Delta_{k}\right)^{-1} P_{l} u\right)_{l \in \mathbb{N}}$ converges strongly to $(\lambda+$ $\left.\Delta_{k}\right)^{-1} u$. Since $\left(\lambda+\Delta_{k}\right)^{-1}$ is also continuous from $L^{q}(F)$ to $W^{2, q}(F),((\lambda+$ $\left.\left.\Delta_{k}\right)^{-1} P_{l} u P_{l}\left(\lambda+\Delta_{k}\right)^{-1}\right)_{l \in \mathbb{N}}$ converges uniformly to $\left(\lambda+\Delta_{k}\right)^{-1} u\left(\lambda+\Delta_{k}\right)^{-1}$ as a compact operator from $L^{q}(F)\left(L^{2}(F)\right.$ in particular) to $L^{2}(F)$. By induction and Neumann's theorem, the same is true for

$$
\left(\left(\lambda+\Delta_{k}\right)^{-1}\left(P_{l} u P_{l}\left(\lambda+\Delta_{k}\right)^{-1}\right)^{n}\right)_{l \in \mathbb{N}}
$$

uniformly for all $n \in \mathbb{N}$, provided we take $u \in B_{C}^{r}$. This proves the approximation claim and hence the weak convergence claim as well. Since $V$ is bounded, we also have uniformity with respect to $k$.

Proposition 3.3.23. Let $d=2$. Then for all $u_{0} \in \mathcal{F} \ell^{\infty, 1}\left(\Gamma^{*}\right)$ there is an $N \in \mathbb{N}$ and $a C>0$ with $u_{0} \in B_{C}^{N}$ such that for all bounded $V \subseteq \mathbb{C}^{d}$ there is a $\lambda_{0} \in$ $(-\infty,-1]$ such that the resolvent $\left(\lambda+\Delta_{k}-u\right)^{-1}$ is defined and maps $\mathcal{F} \ell^{\infty, 1}\left(\Gamma^{*}\right)$ boundedly into $\mathcal{F} \ell^{1}\left(\Gamma^{*}\right)$, and also $L^{2}(F)$ compactly into itself, for all $k \in V$, $u \in B_{C}^{N}$ and all $\lambda \leq \lambda_{0}$. Moreover, the map

$$
\begin{aligned}
B_{C}^{N} \times V & \rightarrow \mathcal{K}\left(L^{2}(F), L^{2}(F)\right) \\
(u, k) & \mapsto\left(\lambda+\Delta_{k}-u\right)^{-1}
\end{aligned}
$$

is continuous with respect to the weak topology on $B_{C}^{N}$ and the strong topologies on $V$ and $\mathcal{K}\left(L^{2}(F), L^{2}(F)\right)$, for all $\lambda \leq \lambda_{0}$. Continuity is uniform with respect to $k$.

Proof. By propositions 3.3.17 and 3.3.19 there is an $N \in \mathbb{N}$ and a $C>0$ with $u_{0} \in B_{C}^{N}$ such that the operator $\left(\Delta_{k}-\Delta_{0}\right)\left(\lambda+\Delta_{0}-u\right)^{-1}$ maps $\mathcal{F} \ell^{\infty, 1}\left(\Gamma^{*}\right)$ boundedly into itself for all $u \in B_{C}^{N}$, with vanishing bound as $\lambda \rightarrow-\infty$. Hence, there is a $\lambda_{0} \in(-\infty,-1]$ such that

$$
\left\|\left(\Delta_{k}-\Delta_{0}\right)\left(\lambda+\Delta_{0}-u\right)^{-1}\right\|<1
$$


for all $u \in B_{C}^{N}$ and all $\lambda \leq \lambda_{0}$. Therefore, the resolvent $\left(\lambda+\Delta_{k}-u\right)^{-1}$ exists and maps $\mathcal{F} \ell^{\infty, 1}\left(\Gamma^{*}\right)$ boundedly into $\mathcal{F} \ell^{1}\left(\Gamma^{*}\right)$ by the construction $(3.3 .22)$ and proposition 3.3 .15 for all $u \in B_{C}^{N}$. Given that $\mathcal{F} \ell^{2}\left(\Gamma^{*}\right)=L^{2}(F)$ by Parseval's theorem and hence $\mathcal{F} \ell^{1}\left(\Gamma^{*}\right) \subseteq L^{2}(F) \subseteq \mathcal{F} \ell^{\infty, 1}\left(\Gamma^{*}\right)$ boundedly, this resolvent is also a compact operator on $L^{2}(F)$ by a further appeal to propositions 3.3 .17 and 2.2.4. The remaining statements follow in the same manner as in the proof of proposition 3.3 .21 .

We can now extend the construction of the full resolvent to nearly all $\lambda \in \mathbb{C}$.

Theorem 3.3.24. Let $d \geq 2$, then for all $u_{0} \in L^{d / 2}(F)$ (resp. $\mathcal{F} \ell^{\infty, 1}\left(\Gamma^{*}\right)$ if $d=2$ ) there is an open neighbourhood $U$ of both $u_{0}$ and 0 which is invariant under translation operators such that for all $(k, u) \in \mathbb{C}^{d} \times U$ there is a discrete set $S(k, u) \subseteq \mathbb{C}$ of finite-order singularities of the resolvent. In particular, the map

$$
\begin{aligned}
\left\{(u, k, \lambda) \in U \times \mathbb{C}^{d} \times \mathbb{C}: \lambda \notin S(k, u)\right\} & \rightarrow \mathcal{K}\left(L^{2}(F), L^{2}(F)\right) \\
(u, k, \lambda) & \mapsto\left(\lambda+\Delta_{k}-u\right)^{-1}
\end{aligned}
$$

is defined and continuous with respect to the weak topology on $U$ and the usual norm topologies otherwise. Continuity is locally uniform in $k$.

Proof. By propositions 3.3.21 and 3.3.23, there is an open neighbourhood $U$ of both $u_{0}$ and 0 which is invariant under translation operators such that for all bounded $V \subseteq \mathbb{C}^{d}$ the resolvent $\left(\lambda_{0}+\Delta_{k}-u\right)^{-1}$ has the desired properties for all $(u, k) \in U \times V$ and some fixed $\lambda_{0} \in(-\infty,-1]$ depending on $V$. Due to the Riesz-Schauder theorem 2.2.7. $\operatorname{Spec}\left(\left(\lambda_{0}+\Delta_{k}-u\right)^{-1}\right)$ contains at most one accumulation point in $\mathbb{C}$, namely zero. The other points are finite-order eigenvalues. Therefore, the set

$$
S(k, u):=\left\{\lambda \in \mathbb{C} \backslash\left\{\lambda_{0}\right\}:\left(\lambda_{0}-\lambda\right)^{-1} \in \operatorname{Spec}\left(\left(\lambda_{0}+\Delta_{k}-u\right)^{-1}\right)\right\}
$$

is discrete. Now, for any $\lambda \in \mathbb{C} \backslash S(k, u), \lambda \neq \lambda_{0}$, the number $\left(\lambda_{0}-\lambda\right)^{-1}$ is in the resolvent set of $\left(\lambda_{0}+\Delta_{k}-u\right)^{-1}$, so the operator

$$
\left(\lambda_{0}-\lambda\right)^{-1}-\left(\lambda_{0}+\Delta_{k}-u\right)^{-1}
$$

is boundedly invertible. This yields

$$
\begin{aligned}
& \left(\lambda_{0}-\lambda\right)^{-1}\left(\lambda_{0}+\Delta_{k}-u\right)^{-1}\left(\left(\left(\lambda_{0}-\lambda\right)^{-1}-\left(\lambda_{0}+\Delta_{k}-u\right)^{-1}\right)^{-1}\right. \\
& =\left(\left(\left(\lambda_{0}-\lambda\right)^{-1}-\left(\lambda_{0}+\Delta_{k}-u\right)^{-1}\right)\left(\lambda_{0}-\lambda\right)\left(\lambda_{0}+\Delta_{k}-u\right)\right)^{-1} \\
& \quad=\left(\lambda_{0}+\Delta_{k}-u-\left(\lambda_{0}-\lambda\right)\right)^{-1}=\left(\lambda+\Delta_{k}-u\right)^{-1}
\end{aligned}
$$

This shows that the map $(u, k, \lambda) \mapsto\left(\lambda+\Delta_{k}-u\right)^{-1}$ is defined. The continuity statements follow from proposition 3.3 .21 .

It remains to show that this construction extends from $V$ to the entire $\mathbb{C}^{d}$. For this purpose, we cover $\mathbb{C}^{d}$ with bounded sets. It is sufficient to show that for some bounded $\widetilde{V} \subseteq \mathbb{C}^{d}$ with $V \cap \widetilde{V} \neq \emptyset$, the corresponding construction with

$$
\widetilde{S}(k, u):=\left\{\lambda \in \mathbb{C} \backslash\left\{\widetilde{\lambda}_{0}\right\}:\left(\widetilde{\lambda}_{0}-\lambda\right)^{-1} \in \operatorname{Spec}\left(\left(\widetilde{\lambda}_{0}+\Delta_{k}-u\right)^{-1}\right\},\right.
$$

where $\widetilde{\lambda}_{0}$ serves the role of $\lambda_{0}$, only this time for $\widetilde{V}$, is compatible. Since compact operators have finite spectral radius, we need only show that $S(k, u)=\widetilde{S}(k, u)$ 
for all $k \in V \cap \tilde{V}$. So let $k \in V \cap \widetilde{V}$ and $\lambda \in S(k, u)$, then $\left(\lambda_{0}-\lambda\right)^{-1}$ is an eigenvalue of $\left(\lambda_{0}+\Delta_{k}-u\right)^{-1}$, so we have for an appropriate eigenfunction $f$ :

$$
\left(\lambda_{0}+\Delta_{k}-u\right)^{-1} f=\frac{1}{\lambda_{0}-\lambda} f
$$

and hence

$$
\left(\lambda_{0}-\lambda\right) f=\left(\lambda_{0}+\Delta_{k}-u\right) f
$$

We can now simply replace $\lambda_{0}$ with $\widetilde{\lambda}_{0}$ on both sides:

$$
\left(\widetilde{\lambda}_{0}-\lambda\right) f=\left(\widetilde{\lambda}_{0}+\Delta_{k}-u\right) f
$$

Now, $\widetilde{\lambda}_{0}$ is in the resolvent set of the Schrödinger operator, so $\lambda \neq \widetilde{\lambda}_{0}$ and

$$
\left(\widetilde{\lambda}_{0}+\Delta_{k}-u\right)^{-1} f=\frac{1}{\widetilde{\lambda}_{0}-\lambda} f .
$$

Hence, $\left(\widetilde{\lambda}_{0}-\lambda\right)^{-1} \in \operatorname{Spec}\left(\left(\widetilde{\lambda}_{0}+\Delta_{k}-u\right)^{-1}\right)$ and thus $\lambda \in \widetilde{S}(k, u)$. This implies $S(k, u) \subseteq \widetilde{S}(k, u)$. By symmetry, the reverse inclusion also holds. 


\section{Chapter 4}

\section{Fermi curves}

The pairs $(k, \lambda) \in \mathbb{C}^{d} \times \mathbb{C}$ (recall that $d \geq 2$ denotes the dimension) for which the Schrödinger equation $\left(-\Delta_{k}+u\right) \psi=\lambda \psi$ has a non-trivial solution make up the Bloch variety ${ }^{1}$ The results from chapter 3 allow us to locally give a precise analytical description of the Bloch variety essentially as the "determinant" of the resolvent of the Schrödinger operator.

For this work, we are especially interested in the fixed-energy subsets of the Bloch variety, the Fermi curves. We shall perform an asymptotic analysis of Fermi curve geometry (that is, investigate the large- $k$ behaviour) in the case $d=$ 2.

\subsection{Bloch varieties and Fermi curves}

For the Schrödinger equation 3.1.8, we tentatively define the Bloch variety

$$
B(u):=\left\{(k, \lambda) \in \mathbb{C}^{d} \times \mathbb{C}: \text { There is a } \psi \neq 0 \text { such that }\left(-\Delta_{k}+u\right) \psi=\lambda \psi\right\} .
$$

The Bloch variety encodes what might be termed the extended spectrum of the Schrödinger operator or, if we neglect branch points, the band functions $\lambda(k)$. Considering the Bloch variety as a mapping $u \mapsto B(u)$, we are interested in the answers to the following two questions:

- The moduli problem: What values $B(u)$ are possible and how do we parameterise them when running through all possible potentials $u$ ?

- The isospectral problem: How can a fibre $B^{-1}\left(B\left(u_{0}\right)\right)$ for some fixed potential $u_{0}$ be parameterised?

While we do not answer these questions in the current thesis, we execute the analytical groundwork to arrive at an apt definition of the Bloch variety to work on these problems. The crucial input here is the compactness of the Schrödinger resolvent on $L^{2}(F)$ for a certain function space of potentials (depending on $d$ ), as derived in theorem 3.3.24. This theorem also establishes some continuity statements. This naturally raises the question of how good this continuity is, that is, how stable the Bloch variety $B(u)$ is under small changes of $u$. We refer

\footnotetext{
${ }^{1}$ Apparently, the term "Bloch variety" was coined by Knörrer and Trubowitz KT90, probably as a tribute to the physicist and Nobel laureate Felix Bloch.
} 
the reader to [FKT90] for a precise analysis of this question (albeit for more regular potentials). However, the following simple statement about perturbations of the free Schrödinger operator can be proven easily:

Proposition 4.1.1. There is a $C>0$ such that for all potentials $u \in L^{d / 2}(F)$ (resp. $u \in \mathcal{F} \ell^{\infty, 1}\left(\Gamma^{*}\right)$ if $d=2$ ), all boundary conditions $k \in \mathbb{C}^{d}$ and all eigenvalues $\lambda$ (with an eigenvector $\psi \in W^{2, q}(F), 1<q<d / 2$, if $d>2, \psi \in \mathcal{F} \ell^{1}\left(\Gamma^{*}\right)$ if $d=2$ as suggested by the results of chapter 3) of the Schrödinger operator $-\Delta_{k}+u$ there is an eigenvalue $\lambda_{0}$ of the free Schrödinger operator $-\Delta_{k}$ satisfying the inequality $\left|\lambda-\lambda_{0}\right| \leq C\|u\|$.

Proof. Let $\lambda$ be an eigenvalue of the Schrödinger operator $-\Delta_{k}+u$ with respect to a normalised eigenvector $\psi$. If $\lambda$ is an eigenvalue of the free Schrödinger operator $-\Delta_{k}$, there is nothing to prove. Otherwise, $\lambda$ is in the resolvent set of $-\Delta_{k}$. In this case, the Schrödinger equation yields the identity

$$
\psi=\left(\lambda+\Delta_{k}\right)^{-1} u \psi .
$$

Now, if $d>2$ then

$$
L^{d / 2}(F) \cdot W^{2, q}(F) \rightarrow L^{q}(F)
$$

boundedly by Sobolev's theorem 2.2.3 and Hölder's inequality (theorem 2.1.1). If $d=2$ then

$$
\mathcal{F} \ell^{\infty, 1}\left(\Gamma^{*}\right) \cdot \mathcal{F} \ell^{1}\left(\Gamma^{*}\right) \rightarrow \mathcal{F} \ell^{\infty, 1}\left(\Gamma^{*}\right)
$$

by proposition 2.4.15. Therefore, taking the norm of both sides of 4.1.2 and using propositions 3.3.21 and 3.3 .23 in the case $u_{0}=0$ yields

$$
1 \leq C^{\prime}\left\|\left(\lambda+\Delta_{k}\right)^{-1}\right\|\|u\|
$$

for some $C^{\prime}>0$. Now, by theorem 3.3.24 (again in the case $u_{0}=0$ ) there is some $C>0$ such that

$$
1 \leq C\left\|\left(\lambda+\Delta_{k}\right)^{-1}\right\|_{L^{2}(F) \rightarrow L^{2}(F)}\|u\| .
$$

Here, $\left(\lambda+\Delta_{k}\right)^{-1}$ is a compact operator on $L^{2}(F)$. Furthermore, $\Delta_{k}$ is normal by proposition 3.1.7. Hence the spectral theorem for compact, normal operators on Hilbert spaces together with the discreteness of the spectrum of $\Delta_{k}$ implies that there is an eigenvalue $\lambda_{0} \in \mathbb{C}$ of $-\Delta_{k}$ such that $\left\|\left(\lambda+\Delta_{k}\right)^{-1}\right\|=\left|\lambda-\lambda_{0}\right|^{-1}$. Therefore, we have

$$
1 \leq C \frac{\|u\|}{\left|\lambda-\lambda_{0}\right|},
$$

proving the proposition.

We shall now locally underlay our tentative definition of Bloch variety using analytically sound concepts. In particular, we shall identify local parts of the Bloch variety with the zero locus of the determinant of a Schrödinger operator which has been appropriately shifted and projected onto a finite-dimensional space.

Theorem 4.1.3. Let $u_{0} \in L^{d / 2}(F)$ (resp. $u_{0} \in \mathcal{F} \ell^{\infty, 1}\left(\Gamma^{*}\right)$ if $d=2$ ), $k_{0} \in \mathbb{C}^{d}$ and $\lambda_{0} \in \mathbb{C}$ such that $\left(u_{0}, k_{0}, \lambda_{0}\right) \in\left(u_{0}, B\left(u_{0}\right)\right)$, that is, there is a non-zero $\psi \in W^{2, q}(F)$ for some $1<q<d / 2$ (resp. $\psi \in \mathcal{F} \ell^{1}\left(\Gamma^{*}\right)$ if $d=2$ ) such that 
$\left(-\Delta_{k_{0}}+u_{0}\right) \psi=\lambda_{0} \psi$. Then there are open neighbourhoods $U, V$ and $W$ of $u_{0}, k_{0}$ and $\lambda_{0}$, respectively, such that for all $(u, k, \lambda) \in U \times V \times W$ there is a finite-dimensional subspace $\Sigma(k, u)$ of $L^{2}(F)$ independent of $\lambda$ which is invariant under the Schrödinger operator $\left(-\Delta_{k}+u\right)$, such that the intersection of the graph of the map $u \mapsto B(u)$ with $U \times V \times W$ is the zero locus of the determinant

$$
(u, k, \lambda) \mapsto \operatorname{det}\left(\left.\left(\lambda+\Delta_{k}-u\right)\right|_{\Sigma(k, u)}\right) .
$$

This map is holomorphic in $\lambda$ and $k$, and continuous in $u$, with the weak topology on $U$ and the usual topologies elsewhere.

Proof. By theorem 3.3.24, $\lambda_{0}$ is an isolated pole of the resolvent $\left(\lambda+\Delta_{k_{0}}-u_{0}\right)^{-1}$. By the reverse Riesz-Schauder theorem 2.2.8, the operator

$$
P\left(k_{0}, u_{0}\right):=\frac{1}{2 \pi i} \oint_{\lambda_{0}}\left(\lambda+\Delta_{k_{0}}-u_{0}\right)^{-1} \mathrm{~d} \lambda
$$

is a $\left(-\Delta_{k_{0}}+u_{0}\right)$-invariant projector. Now, since the resolvent is compact, so is $P\left(k_{0}, u_{0}\right)$. By proposition 2.2.5, $P\left(k_{0}, u_{0}\right)$ has finite rank, so its image $\Sigma\left(k_{0}, u_{0}\right)$ is finite-dimensiona ${ }^{2}$ Let $f_{1}, \ldots, f_{m}$ be a basis of $\Sigma\left(k_{0}, u_{0}\right)$. Let $P^{*}\left(k_{0}, u_{0}\right)$ denote the Hermitian adjoint of $P\left(k_{0}, u_{0}\right)$, then the determinant of the matrix defined by the matrix elements

$$
\left\langle P^{*}\left(k_{0}, u_{0}\right) f_{i} \mid P\left(k_{0}, u_{0}\right) f_{j}\right\rangle,
$$

where $\langle\cdot \mid \cdot\rangle$ denotes the Hermitian form of the Hilbert space $L^{2}(F)$, is nonzero. Now, theorem 3.3 .24 also tells us that if we take some bounded, open neighbourhood of $k_{0}$, there is a certain open neighbourhood of $u_{0}$ such that the resolvent is continuous with respect to $u$ and $k$ from these neighbourhoods. The determinant is continuous as well. Therefore, there is a small open neighbourhood $U$ of $u_{0}$, a small open neighbourhood $V$ of $k_{0}$ and an appropriate $c>0$ such that for all $u \in U$ and all $k \in V$ the operators

$$
P(k, u):=\frac{1}{2 \pi i} \oint_{\left|\lambda-\lambda_{0}\right|=c}\left(\lambda+\Delta_{k}-u\right)^{-1} \mathrm{~d} \lambda
$$

are $\left(-\Delta_{k}+u\right)$-invariant projectors of constant rank to finite-dimensional subspaces $\Sigma(k, u)$ such that the determinants of the matrices defined by

$$
\left\langle P^{*}(k, u) f_{i} \mid P(k, u) f_{j}\right\rangle
$$

are also nonzero. In particular, $P(k, u) f_{1}, \ldots, P(k, u) f_{m}$ is a basis of $\Sigma(k, u)$ for all $u \in U$ and all $k \in V$. Note that this does not necessarily mean that the multiplicity of $\lambda_{0}$ is preserved in a single eigenvalue. Rather, the small open neighbourhood $W:=B_{c}\left(\lambda_{0}\right)$ may contain several eigenvalues split off

\footnotetext{
${ }^{2}$ A further, less general but possibly more intuitive, way to see this is as follows. The image of $P\left(k_{0}, u_{0}\right)$ equals the kernel of $\left(\lambda_{0}+\Delta_{k_{0}}-u_{0}\right)^{n}$ (as an $L^{2}$-operator) for sufficiently large $n \in \mathbb{N}$. For $u_{0}=0$, proposition 2.1.6 shows that the kernel of $\left(\lambda_{0}+\Delta_{k_{0}}\right)$ is at most 2dimensional. Furthermore, $\Delta_{0}$ has discrete eigenvalues. Hence, as shown by proposition 4.1.1 the perturbation on the eigenvalues induced by non-zero potentials $u_{0}$ is at worst proportional to $\left\|u_{0}\right\|$, the kernel of $\left(\lambda_{0}+\Delta_{k_{0}}-u_{0}\right)^{n}$ remains a finite-dimensional subspace $\Sigma\left(k_{0}, u_{0}\right)$ of $L^{2}(F)$. Of course, compactness of the resolvent was a crucial input to proposition 4.1 .1
} 
from $\lambda_{0}$. Due to the invariance of the subspaces $\Sigma(k, u)$, there are matrix elements $\left(A_{i j}(u, k, \lambda)\right)_{i, j=1}^{m}$ of $\lambda+\Delta_{k}-u$ with respect to $P(k, u) f_{1}, \ldots, P(k, u) f_{m}$ defined by

$$
\left(\lambda+\Delta_{k}-u\right) P(k, u) f_{i}=\sum_{j=1}^{m} A_{i j}(u, k, \lambda) P(k, u) f_{j}
$$

for all $i=1, \ldots, m$. The matrix $A(u, k, \lambda)$ defined by these matrix elements is singular if and only if the triple $(u, k, \lambda)$ lies in the graph of $u \mapsto B(u)$. Now,

$$
\left\langle P^{*}(k, u) f_{l} \mid\left(\lambda+\Delta_{k}-u\right) P(k, u) f_{i}\right\rangle=\sum_{j=1}^{m} A_{i j}(u, k, \lambda)\left\langle P^{*}(k, u) f_{l} \mid P(k, u) f_{j}\right\rangle
$$

for all $i, l=1, \ldots, m$. Since the matrix defined by the elements

$$
\left\langle P^{*}(k, u) f_{l} \mid P(k, u) f_{j}\right\rangle
$$

is regular, the determinant of $\left.\left(\lambda+\Delta_{k}-u\right)\right|_{\Sigma(k, u)}$ is zero if and only if the triple $(u, k, \lambda)$ lies in the graph of $u \mapsto B(u)$.

Note that the matrix $A(u, k, \lambda)$ in the above proof is not necessarily diagonalisable. While this will not really cause trouble, it does lead to certain differences between the algebraic and the geometric view of the Schrödinger operator. This theorem is the standard way of describing the Bloch variety, actually a rather basic one using finite-dimensional linear algebra. See Kuc93 for a more sophisticated treatment.

As the title of this thesis suggests, we are mainly interested in a fixed energy slice of the Bloch variety in the case $d=2$. For this purpose, we define

$$
F(u):=\left\{k \in \mathbb{C}^{2}:(k, 0) \in B(u)\right\} .
$$

By theorem 4.1.3, $F(u)$ is a complex curve embedded into $\mathbb{C}^{2}$, called the Fermi curve. It is sufficient to consider only the single fixed energy level $\lambda=0$ because adding a constant to the potential has the effect of subtracting the same constant from the eigenvalues, so

$$
B(u)=\coprod_{\lambda \in \mathbb{C}} F(u-\lambda)
$$

as a set. We shall now investigate the geometry of Fermi curves for $d=2$, first for $u=0$, then asymptotically for general $u$ both away and near certain critical points.

\subsection{The free Fermi curve at $d=2$}

From now on, we let $d=2$. In this section, we construct the free Fermi curve $F(0)$, that is, the set of those $k \in \mathbb{C}^{2}$ for which the free Schrödinger operator $-\Delta_{k}$ has a non-trivial kernel. It is convenient to factorise $-\Delta_{k}$ into a product of first-order differential operators. To this end, we introduce the Wirtinger operators

$$
\partial:=\frac{1}{2}\left(\frac{\partial}{\partial x_{1}}-i \frac{\partial}{\partial x_{2}}\right), \quad \bar{\partial}:=\frac{1}{2}\left(\frac{\partial}{\partial x_{1}}+i \frac{\partial}{\partial x_{2}}\right) .
$$


In analogy to 3.1.4, we can add boundary conditions $k \in \mathbb{C}^{2}$ to the Wirtinger operators:

$$
\partial_{k}:=\partial+\pi i k_{1}+\pi k_{2}, \quad \bar{\partial}_{k}:=\bar{\partial}+\pi i k_{1}-\pi k_{2} .
$$

Lemma 4.2.2. For all $k \in \mathbb{C}^{2}$, we have the operator identity

$$
\partial_{k} \bar{\partial}_{k}=\bar{\partial}_{k} \partial_{k}=\frac{1}{4} \Delta_{k}
$$

Proof. The operators

$$
\partial_{1}:=\frac{\partial}{\partial x_{1}} \quad \text { and } \quad \partial_{2}:=\frac{\partial}{\partial x_{2}}
$$

are commutative in the sense of distributions. Therefore, we have

$$
\begin{aligned}
\bar{\partial}_{k} \partial_{k}= & \frac{1}{4}\left(\left(\partial_{1}-i \partial_{2}+2 \pi i k_{1}+2 \pi k_{2}\right)\left(\partial_{1}+i \partial_{2}+2 \pi i k_{1}-2 \pi k_{2}\right)\right) \\
= & \frac{1}{4}\left(\left(\partial_{1}-i \partial_{2}\right)\left(\partial_{1}+i \partial_{2}\right)-4 \pi^{2}\left(k_{1}-i k_{2}\right)\left(k_{1}+i k_{2}\right)\right. \\
& \left.+2 \pi i\left(\partial_{1}-i \partial_{2}\right)\left(k_{1}+i k_{2}\right)+2 \pi i\left(k_{1}-i k_{2}\right)\left(\partial_{1}+i \partial_{2}\right)\right) \\
= & \frac{1}{4}\left(\partial_{1}^{2}+\partial_{2}^{2}-4 \pi^{2}\left(k_{1}^{2}+k_{2}^{2}\right)\right) \\
& +2 \pi i\left(k_{1} \partial_{1}+i k_{2} \partial_{1}-i k_{1} \partial_{2}+k_{2} \partial_{2}+k_{1} \partial_{1}-i k_{2} \partial_{1}+i k_{1} \partial_{2}+k_{2} \partial_{2}\right) \\
= & \frac{1}{4}\left(\Delta-4 \pi^{2} k^{2}+4 \pi i\left(k_{1} \partial_{1}+k_{2} \partial_{2}\right)\right) \\
= & \frac{1}{4}\left(\Delta-4 \pi^{2} k^{2}+4 \pi i(k \mid \nabla)\right) .
\end{aligned}
$$

The claim now follows from lemma 3.1.5.

Apart from being first-order, these Wirtinger operators with boundary condition have the further advantage of possessing some transformation properties the operator $\Delta_{k}$ does not have.

Definition 4.2.3. For all $\kappa \in \Gamma^{*}$, we set

$$
k_{\kappa}^{\mp}:=\frac{1}{2}\left( \pm \kappa_{1}+i \kappa_{2},-i \kappa_{1} \pm \kappa_{2}\right) .
$$

Note that $k_{\kappa}^{-}-k_{\kappa}^{+}=\kappa$, so $k_{\kappa}^{-}$and $k_{\kappa}^{+}$are distinct unless $\kappa=0$, but they are always congruent modulo $\Gamma^{*}$.

Proposition 4.2.4. For all $k \in \mathbb{C}^{2}$ and all $\kappa \in \Gamma^{*}$, we have the following operator identities:

$$
\begin{array}{ll}
\partial_{k}=\partial_{k+k_{\kappa}^{-}}, & \partial_{k+\kappa}=\psi_{-\kappa} \partial_{k} \psi_{\kappa}, \\
\bar{\partial}_{k}=\bar{\partial}_{k+k_{\kappa}^{+}}, & \bar{\partial}_{k+\kappa}=\psi_{-\kappa} \bar{\partial}_{k} \psi_{\kappa} .
\end{array}
$$

Proof. It follows from definition 4.2 .3 that

$$
i k_{\kappa, 1}^{-}+k_{\kappa, 2}^{-}=0, \quad i k_{\kappa, 1}^{+}-k_{\kappa, 2}^{+}=0,
$$

and thus $\partial_{k}=\partial_{k+k_{\kappa}^{-}}$and $\bar{\partial}_{k}=\bar{\partial}_{k+k_{\kappa}^{+}}$. 
As for the other two identities, we have

$$
\begin{aligned}
\psi_{-\kappa} \partial_{k} \psi_{\kappa}=\psi_{-\kappa} \partial \psi_{\kappa}+\pi i k_{1}+\pi k_{2} & =\partial+\pi i\left(\kappa_{1}-i \kappa_{2}\right)+\pi i k_{1}+\pi k_{2} \\
& =\partial+\pi i \kappa_{1}+\pi \kappa_{2}+\pi i k_{1}+\pi k_{2}=\partial_{k+\kappa}
\end{aligned}
$$

and, likewise, $\psi_{-\kappa} \bar{\partial}_{k} \psi_{\kappa}=\bar{\partial}_{k+\kappa}$.

It follows from lemma 4.2 .2 that the Laplace operator with boundary also has the transformation property

$$
\Delta_{k+\kappa}=\psi_{-\kappa} \Delta_{k} \psi_{\kappa},
$$

but it lacks the invariance under shifts by $k_{\kappa}^{-}$and $k_{\kappa}^{+}$which $\partial_{k}$ and $\bar{\partial}_{k}$, respectively, have. We can now easily prove

Theorem 4.2.5. Let

$$
\mathcal{R}:=\left\{k \in \mathbb{C}^{2}: k_{2}-i k_{1}=0 \text { or } k_{2}+i k_{1}=0\right\},
$$

then $F(0)=\mathcal{R}+\Gamma^{*}$. Furthermore, $\mathcal{R}$ is a system of representatives for the quotient $F(0) / \Gamma^{*}$, provided that the pairs of distinct points $\left(k_{\kappa}^{-}, k_{\kappa}^{+}\right)$are identified to double points for all $\kappa \in \Gamma^{*}, \kappa \neq 0$.

Proof. Let $k \in F(0)$. Then there is a function $\psi \neq 0$ such that, by lemma 4.2 .2 .

$$
\partial_{k} \bar{\partial}_{k} \psi=0 .
$$

Since $\psi \neq 0$, there is a $\kappa \in \Gamma^{*}$ such that $\widehat{\psi}(\kappa) \neq 0$. A Fourier transform of 4.2.7) then yields

$$
\left(i\left(\kappa_{1}+k_{1}\right)-\left(\kappa_{2}+k_{2}\right)\right)\left(i\left(\kappa_{1}+k_{1}\right)+\left(\kappa_{2}+k_{2}\right)\right)=0 .
$$

At least one of these factors is zero, so $F(0) \subseteq \mathcal{R}+\Gamma^{*}$.

Now let $k \in \mathcal{R}+\Gamma^{*}$. Then there is a $\kappa \in \Gamma^{*}$ such that

$$
\left(\kappa_{1}+k_{1}\right)-i\left(\kappa_{2}+k_{2}\right)=0 \quad \text { or } \quad\left(\kappa_{1}+k_{1}\right)+i\left(\kappa_{2}+k_{2}\right)=0,
$$

that is, equation (4.2.8) is fulfilled with this $\kappa$. Now take a function $\psi$ whose Fourier transform is $\widehat{\psi}(\nu)=\delta_{\kappa \nu}$, then $\psi$ solves 4.2 .7$)$. It follows that $F(0)=$ $\mathcal{R}+\Gamma^{*}$.

Finally, we need to investigate when $k-k^{\prime} \in \Gamma^{*}$ for $k, k^{\prime} \in \mathcal{R}$. Assume first that $k_{1}-i k_{2}=0$ and $k_{1}^{\prime}-i k_{2}^{\prime}=0$. This implies $\left(k_{1}-k_{1}^{\prime}\right)-i\left(k_{2}-k_{2}^{\prime}\right)=0$. If there is a $\kappa \in \Gamma^{*}$ such that $k-k^{\prime}=\kappa$ then $\kappa_{1}-i \kappa_{2}=0$. Since $\kappa_{1}, \kappa_{2} \in \mathbb{R}$, this can only be the case if $\kappa=0$ and hence $k=k^{\prime}$. A similar situation applies if $k_{1}+i k_{2}=0$ and $k_{1}^{\prime}+i k_{2}^{\prime}=0$. Without restriction, the only remaining case to be considered is $k_{1}-i k_{2}=0$ and $k_{1}^{\prime}+i k_{2}^{\prime}=0$. This yields the two equations

$$
\left(k_{1}+k_{1}^{\prime}\right)-i\left(k_{2}-k_{2}^{\prime}\right)=0 \quad \text { and } \quad\left(k_{1}-k_{1}^{\prime}\right)-i\left(k_{2}+k_{2}^{\prime}\right)=0
$$

and thus

$$
2 k_{1}-\kappa_{1}-i \kappa_{2}=0 \quad \text { and } \quad k_{1}-i\left(2 k_{2}-\kappa_{2}\right)=0 .
$$




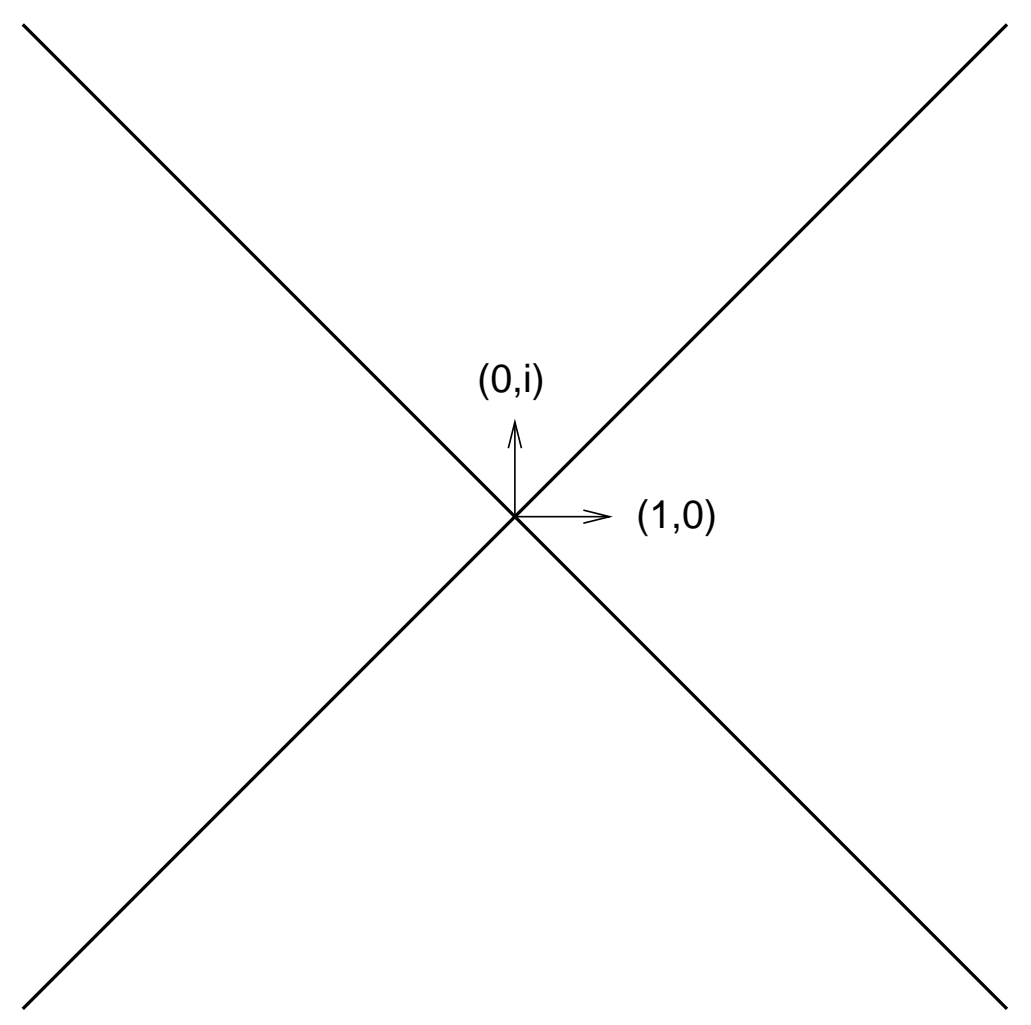

Figure 4.1: Intersection of $\mathcal{R}$ with the real plane spanned by $(1,0)$ and $(0, i)$.

This yields the unique solutions

$$
\begin{aligned}
k_{1} & =\frac{1}{2}\left(\kappa_{1}+i \kappa_{2}\right), & k_{2} & =\frac{1}{2}\left(-i \kappa_{1}+\kappa_{2}\right), \\
k_{1}^{\prime} & =\frac{1}{2}\left(-\kappa_{1}+i \kappa_{2}\right), & k_{2}^{\prime} & =\frac{1}{2}\left(-i \kappa_{1}-\kappa_{2}\right),
\end{aligned}
$$

in accordance with the double points $\left(k_{\kappa}^{-}, k_{\kappa}^{+}\right)$purported in the statement of the theorem.

Subsets of $\mathbb{C}^{2}$ (that's four real dimensions) are somewhat difficult to visualise. However, we can provide some intersections of interesting $\mathbb{C}^{2}$-subsets with real two-dimensional planes. Figure 4.1 shows the intersection of the set of representatives $\mathcal{R}$ (see 4.2.6) with the real two-dimensional plane spanned by $(1,0)$ and $(0, i)$ : two straight lines intersecting at the origin, in accordance with the equations $k_{1}=i k_{2}$ and $k_{1}=-i k_{2}$, respectively. Figure 4.2 shows the entire free Fermi curve $F(0)$ intersected with the same real two-dimensional plane. There are four pairs of double points $\left(k_{\kappa}^{-}, k_{\kappa}^{+}\right)$in the visible part of the intersection as indicated by the small digits. Note that the double points all reside at intersections in figure 4.2 But this is only due to the fact that the corresponding eigenspaces are, as the proof of theorem 4.2 .5 shows, two-dimensional and generated by $\psi_{0}$ and $\psi_{\kappa}$. In what follows, we shall remain with this view of double points. 


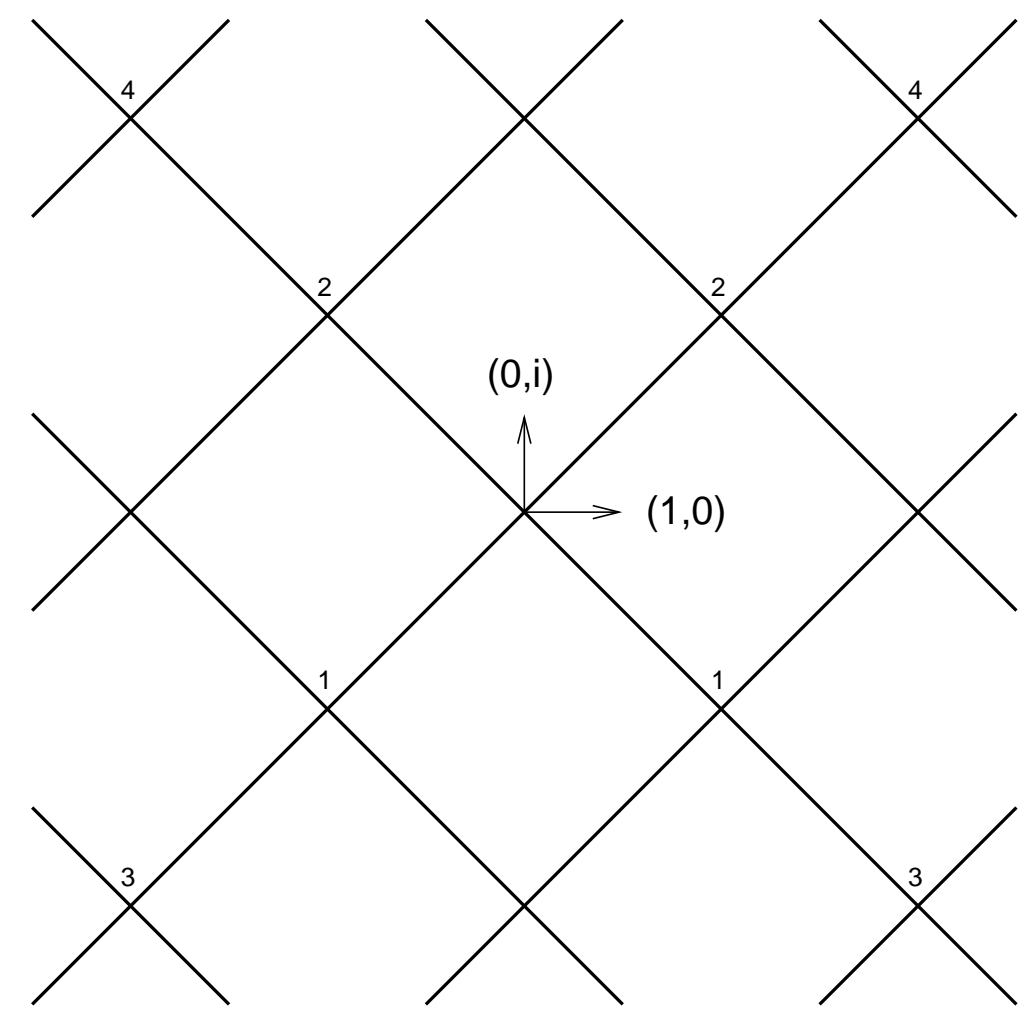

Figure 4.2: Intersection of the free Fermi curve $F(0)$ with respect to the lattice $\Gamma^{*}=$ $4 \mathbb{Z} \oplus 4 \mathbb{Z}$ with the real plane spanned by $(1,0)$ and $(0, i)$. All visible double points $k_{\kappa}^{\mp}$ are indicated with small digits, where equal digits denote the same double point. 
Theorem 4.2.5 in combination with theorem 3.3.24 shows that $\Delta_{k}^{-1}$ exists as a compact operator on $L^{2}(F)$ whenever $k \notin F(0)$. We can also easily prove the following similar proposition.

Proposition 4.2.10. Let $k_{0} \in \mathbb{C}^{2} \backslash F(0)$. Then there is a small open neighbourhood $V$ of $k_{0}$ and a $c>0$ such that the resolvent $\Delta_{k}^{-1}$ exists for all $k \in V$ and is bounded from $\mathcal{F} \ell^{\infty, 1}\left(\Gamma^{*}\right)$ to $\mathcal{F} \ell^{1}\left(\Gamma^{*}\right)$ with a bound smaller than $c$, and compact from $\mathcal{F} \ell^{\infty, 1}$ to $L^{2}(F)$.

Proof. By theorem 4.2.5 $F(0)$ is a closed subset of $\mathbb{C}^{2}$, so we can find an open neighbourhood $V$ of $k_{0}$ bounded away from $F(0)$. The proof of boundedness is now equal to the proof of theorem 3.2 .5 , except that we use

$$
g(\kappa):=\frac{1}{-4 \pi^{2}(k+\kappa)^{2}}
$$

instead of 3.2.6 and, in order to obtain 3.2.8 for suitable $C_{1}, C_{2}>0$, we take an arrangement $\left(\kappa_{n}\right)_{n \in \mathbb{N}}$ such that $\left\|k+\kappa_{n}\right\| \geq\left\|k+\kappa_{n^{\prime}}\right\|$ whenever $n \geq n^{\prime}$. For sufficiently small neighbourhoods $V$, this arrangement is independent of $k$. For the compactness claim, we apply the analogous substitutions to the proof of theorem 3.2.10. This shows that the resolvent is compact from $\mathcal{F} \ell^{\infty, 1}\left(\Gamma^{*}\right)$ to $\mathcal{F} \ell^{2,1}\left(\Gamma^{*}\right) \subseteq L^{2}(F)$.

Naturally, the norm of $\Delta_{k}^{-1}$ becomes arbitrarily large as $k_{0}$ approaches $F(0)$.

\subsection{Asymptotic freeness}

In this section, we investigate the large- $k$ behaviour of Fermi curves $F(u)$ for arbitrary $u \in \mathcal{F} \ell^{\infty, 1}\left(\Gamma^{*}\right)$. From theorem 3.3.24 it is already clear that, locally, the Fermi curves $F(u)$ will be very similar to the free Fermi curve if the $u$ 's are taken from the intersection of a small weakly open (i.e., open with respect to the weak topology) and a large strongly bounded neighbourhood of the zero potential. It is possible to exploit the transformation behaviour of the Wirtinger operators with boundary condition to lift this weakly-local property to a stronglyasymptotic one (with certain reservations) in a purely geometric way. However, since in the Schrödinger operator not all of the properties proven in proposition 4.2.4 are exploitable, we need a new operator with an extended set of Fermi curves containing the Schrödinger theory. One operator which accomplishes this is the two-dimensional Dirac operator. Unfortunately, for technical reasons the resolvent theory of the Dirac operator can only be developed in a very slightly less general setting in terms of available potentials than the resolvent theory of the Schrödinger operator. Therefore, we shall develop the asymptotic theory of Schrödinger Fermi curves directly. Nevertheless, the Dirac theory is very beautiful despite its shortcomings and is therefore presented in appendix $\mathrm{B}$.

For the direct approach, we first need a proper localisation of the Fermi curve. First off, we expect a transformation of boundary conditions $k \mapsto k+\kappa$ to have no effect on the Fermi curve. Indeed, we have

Lemma 4.3.1. For all $u \in \mathcal{F} \ell^{\infty, 1}\left(\Gamma^{*}\right)$ and all $\kappa \in \Gamma^{*}$ the Bloch variety $B(u)$ and the Fermi curve $F(u)$ are invariant under the transformation $k \mapsto k+\kappa$.

\footnotetext{
${ }^{3}$ The Dirac operator has other, more serious shortcomings not immediately related to the coarse asymptotic analysis we are performing in this subsection.
} 
Proof. Let $k \in B(u)$. Then there is a nonzero eigenfunction $\psi$ and an eigenvalue $\lambda \in \mathbb{C}$ such that

$$
-\Delta_{k} \psi+u \psi=\lambda \psi
$$

This yields

$$
-\psi_{-\kappa} \Delta_{k} \psi_{\kappa} \psi_{-\kappa} \psi+u \psi_{-\kappa} \psi=\lambda \psi_{-\kappa} \psi .
$$

Hence, by proposition 4.2.4 the transformation $k \mapsto k+\kappa$ merely induces a transformation of the eigenfunction. This proves the statements.

This lemma shows that we can safely restrict our attention to the quotients $F(u) / \Gamma^{*}$. Nevertheless, this transition to the quotient still has repercussions with respect to the weak perturbations alluded to above as soon as one identifies the set $\mathbb{C}^{2} / \Gamma^{*}$ with a closed fundamental domain in $\mathbb{C}^{2}$ (that is, one takes a fundamental domain and takes the closure of it). Take the free Fermi curve $F(0)$, for example. As already mentioned, a perturbation of the resolvent with a small potential $u$ leads to a Fermi curve $F(u)$ which locally deviates only little from $F(0)$ with respect to any metric which behaves as can be geometrically expected. One such metric is the Hausdorff metric for subsets. Let $V \subseteq \mathbb{C}^{2}$ be bounded, such that

$$
V_{1}:=V \cap F(0), \quad V_{2}:=V \cap F(u)
$$

are not empty, then the Hausdorff distance of the parts $V_{1}, V_{2}$ of these Fermi curves that lie within $V$ is given by

$$
d_{H}\left(V_{1}, V_{2}\right):=\max \left\{\sup _{k \in V_{1}} \inf _{k^{\prime} \in V_{2}}\left\|k-k^{\prime}\right\|, \sup _{k \in V_{2}} \inf _{k^{\prime} \in V_{1}}\left\|k-k^{\prime}\right\|\right\} .
$$

At least for fixed $V$, the Hausdorff distance becomes arbitrarily small for arbitrarily small perturbations of the zero potential. This is no longer true after the quotients $F(0) / \Gamma^{*}$ and $F(u) / \Gamma^{*}$ have been identified with proper systems of representatives in a closed fundamental domain in $\mathbb{C}^{2}$. Due to theorem 4.2 .5 , we shall take $\mathcal{R}$ as the system of representatives for $F(0) / \Gamma^{*}$. Alas, $\mathcal{R}$ contains the double points $k_{\kappa}^{\mp}$. A perturbation of the zero potential may cause a double point to "split up": the corresponding two-dimensional eigenspace of the two points constituting the double points decays into two distinct one-dimensional eigenspaces (for sufficiently small perturbations, this is the only mutation that can happen because the double points are discrete). This motivates the following definition.

Definition 4.3.2. Let $\kappa \in \Gamma^{*}$. We call a compact set $H \subseteq \mathbb{C}^{2}$ a handle for the double point $k_{\kappa}^{\mp}$ if $H$ is connected and contains open neighbourhoods of both $k_{\kappa}^{-}$ and $k_{\kappa}^{+}$. Furthermore, we call the maximum distance of a point of $H$ from the free Fermi curve $F(0)$ the thickness of $H$.

Figure 4.3, which is based on figure 4.2, shows two examples of handles. So while arbitrarily small perturbations will produce parts of the Fermi curve quotient $F(u) / \Gamma^{*}$ that fit in arbitrarily thin handles, even the smallest perturbation will, in general, require handles for every double point to contain the Fermi curve. Of course, not every perturbation will split every double point. In this section, we are mainly interested in what happens outside the handles. For the inside, see section 4.5 . 


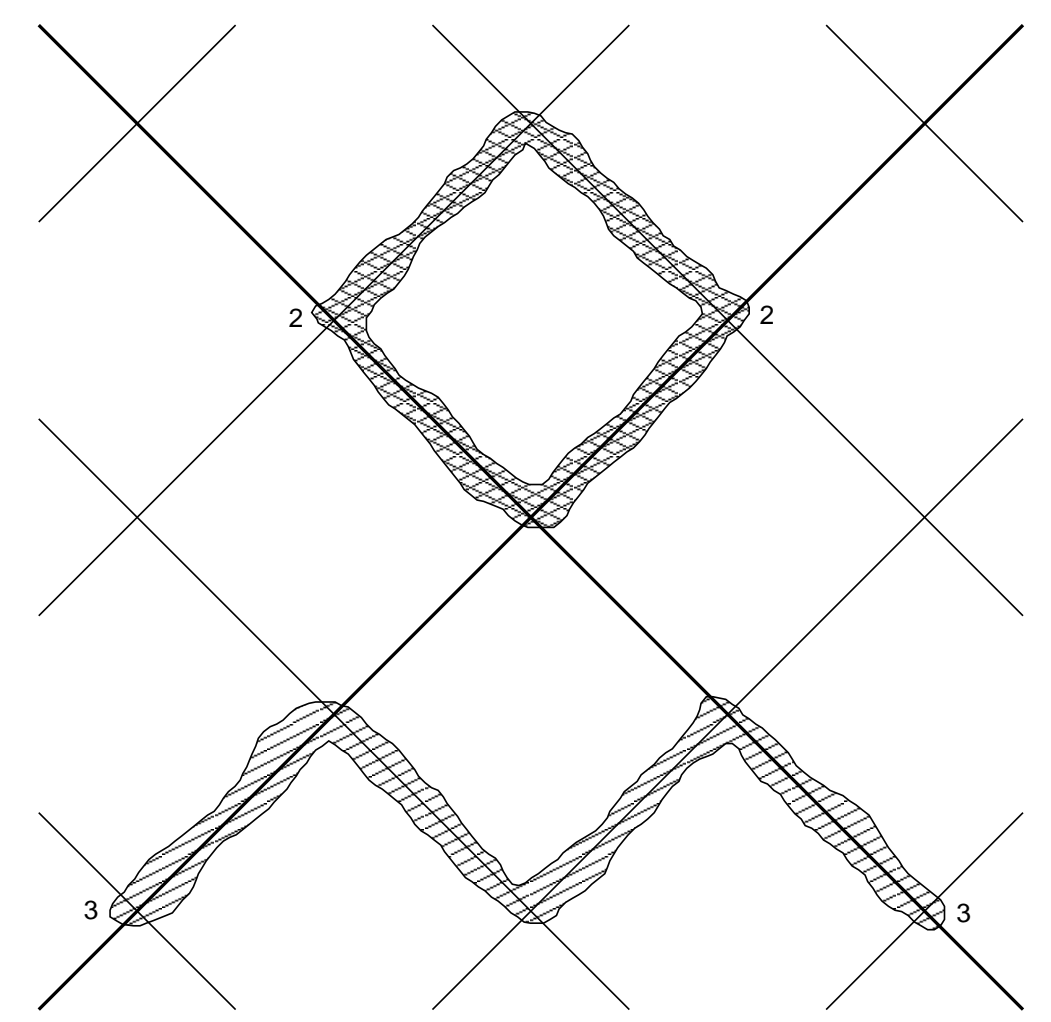

Figure 4.3: Two examples of handles. This figure depicts the representatives $\mathcal{R}$ of the free Fermi curve $F(0)$ (thick lines) with the remainder of the free Fermi curve as backdrop (thin lines), where two pairs of double points are indicated by the digits 2 and 3 , in accordance with figure $4.2\left(\Gamma^{*}=4 \mathbb{Z} \oplus 4 \mathbb{Z}\right.$, intersection with the real two-dimensional plane spanned by $(1,0)$ and $(0, i)$ in particular). The double points " 2 " and " 3 " are connected by handles as indicated by the doubly-hatched and singly-hatched areas, respectively. The thickness of the depicted handles is already relatively small compared with the lattice constant. 
Since $\mathbb{C}^{2}$ has four real dimensions and $\Gamma^{*}$ only two, $\mathbb{C}^{2} / \Gamma^{*}$ is not compact. Therefore, we define

$$
\Gamma_{\mathbb{C}}^{*}:=\left\langle\kappa, k_{\kappa}^{-}\right\rangle_{\kappa \in \Gamma^{*}},
$$

that is, $\Gamma_{\mathbb{C}}^{*}$ is the lattice generated by all lattice vectors of $\Gamma^{*}$ and all the double points $k_{\kappa}^{\mp}$ (since $k_{\kappa}^{-}-k_{\kappa}^{+} \in \Gamma^{*}$, it does not matter whether we use $k_{\kappa}^{-}$or $k_{\kappa}^{+}$in the definition). This is a real four-dimensional lattice, so $\mathbb{C}^{2} / \Gamma_{\mathbb{C}}^{*}$ is compact. It follows from theorem 4.2 .5 that $F(0)$ is still invariant under any of the translations $k \mapsto k_{\kappa}^{\mp}$ for all $\kappa \in \Gamma^{*}$. For general Fermi curves, however, this is no longer true. But it is not needed either for the asymptotic analysis. Let us first make the following basic observation.

Lemma 4.3.3. Let $V \subseteq \mathbb{C}^{2} \backslash F(0)$ be compact, then we have for lattice vectors $\rho, \nu \in \Gamma^{*}$ :

$$
\lim _{\|\nu\| \rightarrow \infty} \inf _{\substack{\rho \in \Gamma^{*} \\ k \in V}}\left|\left(\rho+k+k_{\nu}^{ \pm}\right)^{2}\right|=\infty .
$$

More precisely,

$$
(\nu, \rho) \mapsto \frac{1}{\left(\rho+k+k_{\nu}^{ \pm}\right)^{2}}
$$

defines $c^{0}\left(\Gamma^{*}\right) \widehat{\otimes} \ell^{1, \infty}\left(\Gamma^{*}\right)$-functions holomorphically dependent on $k$ in the interior of $V$.

Proof. Let $\left(\nu_{n}\right)_{n \in \mathbb{N}}$ a sequence in $\Gamma^{*}$ with

$$
\lim _{n \rightarrow \infty}\left\|\nu_{n}\right\|=\infty .
$$

Now,

$$
\begin{aligned}
\left(\rho_{n}+k_{n}+k_{\nu_{n}}^{ \pm}\right)^{2}= & \left(\rho_{n}+k_{n}\right)^{2}+2\left(\rho_{n}+k_{n} \mid k_{\nu_{n}}^{ \pm}\right)+\left(k_{\nu_{n}}^{ \pm}\right)^{2} \\
= & \left(\rho_{n, 1}+k_{n, 1}\right)^{2}+\left(\rho_{n, 2}+k_{n, 2}\right)^{2} \\
& +\left(\rho_{n, 1}+k_{n, 1}\right)\left(\mp \nu_{n, 1}+i \nu_{n, 2}\right) \\
& +\left(\rho_{n, 2}+k_{n, 2}\right)\left(\mp \nu_{n, 2}-i \nu_{n, 1}\right) \\
= & \left(\rho_{n, 1}+k_{n, 1}\right)\left(\rho_{n, 1}+k_{n, 1} \mp \nu_{n, 1}+i \nu_{n, 2}\right) \\
& +\left(\rho_{n, 2}+k_{n, 2}\right)\left(\rho_{n, 2}+k_{n, 2} \mp \nu_{n, 2}-i \nu_{n, 1}\right) .
\end{aligned}
$$

Here, $k_{n}$ and $\rho_{n}$ are vectors such that the infimum is attained. They exist because $V$ is compact, $\Gamma^{*}$ is discrete and $\|\rho\| \rightarrow \infty$ would imply $\left|\left(\rho+k+k_{\nu}^{ \pm}\right)^{2}\right| \rightarrow$ $\infty$. The infimum of $\left|\left(\rho_{n}+k_{n}+k_{\nu_{n}}^{ \pm}\right)^{2}\right|$ can be determined using standard calculus if we extend the domain for the $\rho$. We then obtain the infimum at

$$
\rho+k=\mp \frac{1}{2} \nu,
$$

yielding a value of $\|\nu\|^{2} / 4$, which approaches infinity as $\|\nu\| \rightarrow \infty$. This behaviour remains the same without extending the domain because the imaginary part of $k$ is bounded.

As for the second claim, $\rho$ appears quadratically in the denominator of the map (4.3.4). Since $\Gamma^{*}$ is two-dimensional, this makes this function certainly $\ell^{1, \infty}\left(\Gamma^{*}\right)$. As for the decreasing rearrangement with respect to $\rho$, call it $g^{\star}(k, \nu, n)$, we have the estimate

$$
n g^{\star}(k, \nu, n) \leq C \frac{n}{n+\sqrt{n}\|\nu\|+\|\nu\|^{2}}
$$


for a suitable $C>0$. This shows the $c^{0}\left(\Gamma^{*}\right)$ claim.

Let $F_{\mathbb{C}}$ be a fixed compact fundamental domain for $\mathbb{C}^{2} / \Gamma_{\mathbb{C}}^{*}$. For $\epsilon>0$, we set

$$
F_{\mathbb{C}}^{\epsilon}:=\left\{k \in F_{\mathbb{C}}: \operatorname{dist}(k, F(0)) \geq \epsilon\right\} .
$$

Clearly, $F_{\mathbb{C}}^{\epsilon}$ is closed and hence compact. Furthermore, we define for our convenience

$$
\Gamma_{\delta}^{*}:=\left\{\kappa \in \Gamma^{*}:\|\kappa\|>\delta^{-1}\right\}
$$

for all $\delta>0$. Then we can prove

Lemma 4.3.7. Let $u_{0} \in \mathcal{F} \ell^{\infty, 1}\left(\Gamma^{*}\right)$. Then for all sufficiently small $\epsilon>0$ there is an $N \in \mathbb{N}$ and $a C>0$ with $u_{0} \in B_{C}^{N}$ as well as a $\delta>0$ such that for all $k \in F_{\mathbb{C}}^{\epsilon}$, all $\nu \in \Gamma_{\delta}^{*}$ and all $u \in B_{C}^{N}$ the operator $u \Delta_{k+k_{\nu}^{-}}^{-1}$ exists and is bounded on $\mathcal{F} \ell^{\infty, 1}\left(\Gamma^{*}\right)$ with

$$
\left\|u \Delta_{k+k_{\nu}^{-}}^{-1}\right\|<\epsilon .
$$

Proof. Let $k_{0} \in F_{\mathbb{C}}^{\epsilon}$. By proposition 4.2.10, there is a small open neighbourhood $V$ of $k_{0}$ such that $\Delta_{k}^{-1}$ is bounded from $\mathcal{F} \ell^{\infty, 1}\left(\Gamma^{*}\right)$ to $\mathcal{F} \ell^{1}\left(\Gamma^{*}\right)$ by some constant $c_{V}>0$ for all $k \in V$. We can choose $V$ such that $\operatorname{dist}(V, F(0))>\epsilon / 2$. Due to translation invariance, the same is true for all $k \in V+k_{\nu}^{-}$, for all $\nu \in \Gamma^{*}$. The proof now proceeds in a fashion similar to the proof of lemma 3.3.6. The operator $u \Delta_{k+k_{\nu}}^{-1}$ is bounded on $\mathcal{F} \ell^{\infty, 1}\left(\Gamma^{*}\right)$ for all $u \in \mathcal{F} \ell^{\infty, 1}\left(\Gamma^{*}\right)$, all $k \in V$ and all $\nu \in \Gamma^{*}$. Take

$$
C_{V}:=\frac{\epsilon \mu(F)}{2 c_{V}}
$$

and choose $N_{V} \in \mathbb{N}$ large enough, so $\left\|\widehat{u}_{0}\right\|_{(\infty, 1) ; N_{V}}<C_{V}$. Let $f \in \mathcal{F} \ell^{\infty, 1}\left(\Gamma^{*}\right)$. With a decomposition $u=u_{1}+u_{2}$ as in 3.3 .8 for all $u \in B_{C}^{N_{V}}$, we have the estimate

$$
\begin{aligned}
\sum_{n=1}^{\infty} \frac{1}{n}\left(\kappa \mapsto \sum_{\kappa-\rho \in A_{u}}\left|\frac{\widehat{f}(\rho)}{-4 \pi^{2}\left(k+k_{\nu}^{-}+\rho\right)^{2}}\right|\right)^{\star}(n) & \\
\leq & \frac{N_{V}}{4 \pi^{2} \inf _{\substack{\rho \in \Gamma^{*} \\
k \in F_{\mathbb{C}} / 2}}\left|\left(k+k_{\nu}^{-}+\rho\right)^{2}\right|}\||\widehat{f}|\|_{\infty, 1} .
\end{aligned}
$$

Due to lemma 4.3 .3 , the factor

$$
\frac{N_{V}}{4 \pi^{2} \inf _{\substack{\rho \in \Gamma^{*} \\ k \in F_{\mathrm{C}}^{\epsilon / 2}}}\left|\left(k+k_{\nu}^{-}+\rho\right)^{2}\right|}
$$

is bounded by $\epsilon / 2$ for all $\nu \in \Gamma_{\delta_{V}}^{*}$ with sufficiently small $\delta_{V}>0$. We can repeat this procedure with all $k_{0} \in F_{\mathbb{C}}^{\epsilon}$, gaining the same results for different $V$ s. Since $F_{\mathbb{C}}^{\epsilon}$ is compact, only a finite number of $V \mathrm{~s}$ is required to cover $F_{\mathbb{C}}^{\epsilon}$, so the results uniformly extend to all $F_{\mathbb{C}}^{\epsilon}$ by choosing $N:=\max N_{V}, C:=\min C_{V}$ and $\delta:=\min \delta_{V}$.

This lemma immediately leads to the following theorem. 
Theorem 4.3.8. Let $u_{0} \in \mathcal{F} \ell^{\infty, 1}\left(\Gamma^{*}\right)$. Then for all $\epsilon>0$ there is an $N \in \mathbb{N}$ and $a C>0$ with $u_{0} \in B_{C}^{N}$ as well as a $\delta>0$ such that for all $k \in F_{\mathbb{C}}^{\epsilon}$, all $\nu \in \Gamma_{\delta}^{*}$ and all $u \in B_{C}^{N}$ the operators $\Delta_{k+k_{\nu}^{-}}^{-1}$ and $\left(\Delta_{k+k_{\nu}^{-}}-u\right)^{-1}$ exist and are bounded from $\mathcal{F} \ell^{\infty, 1}\left(\Gamma^{*}\right)$ to $\mathcal{F} \ell^{1}\left(\Gamma^{*}\right)$ with

$$
\left\|\left(\Delta_{k+k_{\nu}^{-}}-u\right)^{-1}-\Delta_{k+k_{\nu}^{-}}^{-1}\right\|<\epsilon .
$$

Proof. By proposition 4.2.10, $\Delta_{k+k_{\nu}^{-}}^{-1}$ exists and is bounded from $\mathcal{F} \ell^{\infty, 1}\left(\Gamma^{*}\right)$ to $\mathcal{F} \ell^{1}\left(\Gamma^{*}\right)$ for all $k \in F_{\mathbb{C}}^{\epsilon}$ and all $\nu \in \Gamma^{*}$. By lemma 4.3.7, for all $0<\epsilon^{\prime} \leq \epsilon$ there are $N \in \mathbb{N}, C>0$ and $\delta^{\prime}>0$ such that

$$
\left\|u \Delta_{k+k_{\nu}^{-}}\right\|_{\mathcal{F} \ell^{\infty, 1}\left(\Gamma^{*}\right) \rightarrow \mathcal{F} \ell^{\infty, 1}\left(\Gamma^{*}\right)}<\epsilon^{\prime}
$$

for all $u \in B_{C}^{N}, k \in F_{\mathbb{C}}^{\epsilon} \subseteq F_{\mathbb{C}}^{\epsilon^{\prime}}$ and all $\nu \in \Gamma_{\delta^{\prime}}^{*}$. For a fixed, sufficiently small $\epsilon^{\prime}$, we can express $\left(\Delta_{k+k_{\nu}^{-}}-u\right)^{-1}$ as a Neumann series (cf. the methods of section 3.3):

$$
\left(\Delta_{k+k_{\nu}^{-}}-u\right)^{-1}=\Delta_{k+k_{\nu}^{-}}^{-1}\left(1-u \Delta_{k+k_{\nu}^{-}}^{-1}\right)^{-1}=\Delta_{k+k_{\nu}^{-}}^{-1} \sum_{n=0}^{\infty}\left(u \Delta_{k+k_{\nu}^{-}}^{-1}\right)^{n} .
$$

This shows that $\left(\Delta_{k+k_{\nu}^{-}}-u\right)^{-1}$ is bounded from $\mathcal{F} \ell^{\infty, 1}\left(\Gamma^{*}\right)$ to $\mathcal{F} \ell^{1}\left(\Gamma^{*}\right)$. Furthermore,

$$
\left\|\left(\Delta_{k+k_{\nu}^{-}}-u\right)^{-1}-\Delta_{k+k_{\nu}^{-}}^{-1}\right\| \leq\left\|\Delta_{k+k_{\nu}^{-}}^{-1}\right\|\left\|\sum_{n=0}^{\infty}\left(u \Delta_{k+k_{\nu}^{-}}^{-1}\right)^{n}-1\right\| \leq\left\|\Delta_{k+k_{\nu}^{-}}^{-1}\right\| \frac{\epsilon^{\prime}}{1-\epsilon^{\prime}} .
$$

Now, $\Delta_{k+k_{\nu}^{-}}^{-1}$ is uniformly bounded because $F_{\mathbb{C}}^{\epsilon}$ is compact and $F(0)$ is invariant under translations by $k_{\kappa}^{-}$. Therefore, the above norm is smaller than $\epsilon$ for all $k \in F_{\mathbb{C}}^{\epsilon}$ and all $\nu \in \Gamma_{\delta^{\prime}}^{*}$ for a sufficiently small $\epsilon^{\prime}$.

In geometric terms, the results of this section can be expressed as follows.

Corollary 4.3.9. Let $u_{0} \in \mathcal{F} \ell^{\infty, 1}\left(\Gamma^{*}\right)$. Then for all $\epsilon>0$ there is an $N \in \mathbb{N}$ and $a C>0$ with $u_{0} \in B_{C}^{N}$ such the Fermi curves $F(u) / \Gamma^{*}, u \in B_{C}^{N}$, consist of three parts, depending only on $\epsilon$ :

1. A part whose distance from $F(0) / \Gamma^{*}$ is globally less than $\epsilon$.

2. A part such that the corresponding part in $\mathbb{C}^{2}$ is contained in a union of handles whose thickness does not exceed $\epsilon$.

3. The remainder, which is contained in a compact subset of $\mathbb{C}^{2} / \Gamma^{*}$.

An even more succinct way to express the geometric behaviour of Fermi curves is: all Fermi curves are asymptotically free outside the handles, and even these have asymptotically vanishing thickness.

\subsection{The constant potential Fermi curve at $d=2$}

In section 4.2 we saw that the free Fermi curve $F(0) / \Gamma^{*}$ at $d=2$ has a very simple $\mathbb{C}^{2}$-representation $\mathcal{R}$, consisting of two $\mathbb{C}$-planes intersecting at the origin. In section 4.3 we saw that for an arbitrary potential $u \in \mathcal{F} \ell^{\infty, 1}\left(\Gamma^{*}\right)$, the 
Fermi curve $F(u) / \Gamma^{*}$ asymptotically has the same representation, except that the double points $\left(k_{\nu}^{-}, k_{\nu}^{+}\right)$inherent in the representation $\mathcal{R}$ split up in general, introducing the necessity of the handles. In the worst (and generic) case, the two simple planes $\mathcal{R}$ mutate to a surface of infinite genus.

If the potential $u$ is constant, the resulting Fermi curve $F(u) / \Gamma^{*}$ is, of course, still asymptotically free, and furthermore this time no handles are needed because the double points do not split. To see how this comes about, consider the formal Fourier series for an arbitrary potential $u$ (cf. (2.1.7)):

$$
u(x)=\frac{1}{\mu(F)} \sum_{\kappa \in \Gamma^{*}} \psi_{\kappa}(x) \widehat{u}(\kappa) .
$$

If $u$ is constant, this simply means that the only Fourier coefficient of $u$ allowed to be nonzero is $\widehat{u}(0)$. In particular, we can write

$$
u(x)=4 \pi^{2} \widehat{u}_{0}+\frac{1}{\mu(F)} \sum_{\kappa \in \Gamma^{*} \backslash\{0\}} \psi_{\kappa}(x) \widehat{u}(\kappa),
$$

where

$$
\widehat{u}_{0}:=\frac{\widehat{u}(0)}{4 \pi^{2} \mu(F)} .
$$

This corresponds to a decomposition of $\mathcal{F} \ell^{\infty, 1}\left(\Gamma^{*}\right)$ to a direct sum of the space of constant potentials with the space of potentials with zero average, because all $\psi_{\kappa}$ except $\psi_{0}$ have zero average. Hence, we can, by lemma 3.1.5, rewrite the Schrödinger operator as

$$
\begin{array}{r}
-\Delta_{k}+u=-\left(\Delta+4 \pi i(k \mid \nabla)-4 \pi^{2} k^{2}\right)+4 \pi^{2} \widehat{u}_{0}+\frac{1}{\mu(F)} \sum_{\kappa \in \Gamma^{*} \backslash\{0\}} \psi_{\kappa}(x) \widehat{u}(\kappa) \\
=-\left(\Delta+4 \pi i(k \mid \nabla)-4 \pi^{2} k^{2}-4 \pi^{2} \widehat{u}_{0}\right)+\left(u-4 \pi^{2} \widehat{u}_{0}\right) .
\end{array}
$$

In other words, we may absorb the constant part of $u$ to form an "extended" boundary condition because it does not depend on $x$, and then consider only potentials with zero average. Note that proposition 3.1.7 also generalises to Schrödinger operators with constant potential. A Schrödinger operator with constant potential is therefore kind of a free Schrödinger operator with a slightly more complicated boundary condition.

Theorem 4.4.1. Let $4 \pi^{2} \widehat{u}_{0}$ be a constant potential and

$$
\mathcal{R}\left(\widehat{u}_{0}\right):=\left\{k \in \mathbb{C}^{2}:\left(k_{2}-i k_{1}\right)\left(k_{2}+i k_{1}\right)+\widehat{u}_{0}=0\right\} .
$$

Then $F\left(4 \pi^{2} \widehat{u}_{0}\right)=\mathcal{R}\left(\widehat{u}_{0}\right)+\Gamma^{*}$. The set $\mathcal{R}\left(\widehat{u}_{0}\right)$ is a system of representatives for $F\left(4 \pi^{2} \widehat{u}_{0}\right) / \Gamma^{*}$, provided that the pairs of distinct points $\left(k_{\kappa}^{-}\left(\widehat{u}_{0}\right), k_{\kappa}^{+}\left(\widehat{u}_{0}\right)\right)$ given by

$$
k_{\kappa}^{\mp}\left(\widehat{u}_{0}\right):=\frac{1}{2}\left( \pm \kappa_{1}+i \kappa_{2} \xi\left(\widehat{u}_{0}, \kappa\right),-i \kappa_{1} \xi\left(\widehat{u}_{0}, \kappa\right) \pm \kappa_{2}\right), \quad \xi\left(\widehat{u}_{0}, \kappa\right):=\sqrt{1+4 \frac{\widehat{u}_{0}}{\kappa^{2}}},
$$

are identified to double points for all $\kappa \in \Gamma^{*}, \kappa \neq 0$, and further provided that the constant potential does not cause double points to coincide? In particular,

\footnotetext{
${ }^{4}$ This can only happen for double points whose norm lies below a bound which depends only on $\widehat{u}_{0}$. Therefore, we can safely ignore this case for our asymptotic analysis. However, it does become important when passing from the Fermi curve to the Bloch variety. See section 5.4 for a short discussion.
} 
$\mathcal{R}\left(\widehat{u}_{0}\right)$ is a continuous deformation of $\mathcal{R}$, and the double points $k_{\kappa}^{\mp}$ of $\mathcal{R}$ remain unsplit and correspond to $k_{\kappa}^{\mp}\left(\widehat{u}_{0}\right)$.

Proof. By applying the Fourier transform on the Schrödinger operator, we see, as in the proof of theorem 4.2.5, that

$$
\begin{aligned}
F\left(4 \pi^{2} \widehat{u}_{0}\right)=\left\{k \in \mathbb{C}^{2}: \text { There is a } \kappa \in \Gamma^{*}\right. \text { such that } \\
\\
\left.\quad\left(\left(k_{2}+\kappa_{2}\right)-i\left(k_{1}+\kappa_{1}\right)\right)\left(\left(k_{2}+\kappa_{2}\right)+i\left(k_{1}+\kappa_{1}\right)\right)+\widehat{u}_{0}=0 .\right\} .
\end{aligned}
$$

This shows $F\left(4 \pi^{2} \widehat{u}_{0}\right)=\mathcal{R}\left(\widehat{u}_{0}\right)+\Gamma^{*}$. For the double points, we must solve the equations

$$
\left(k_{\kappa}^{-}\left(\widehat{u}_{0}\right)\right)^{2}+\widehat{u}_{0}=0, \quad\left(k_{\kappa}^{+}\left(\widehat{u}_{0}\right)\right)^{2}+\widehat{u}_{0}=0, \quad k_{\kappa}^{-}\left(\widehat{u}_{0}\right)-k_{\kappa}^{+}\left(\widehat{u}_{0}\right)=\kappa
$$

for $k_{\kappa}^{\mp}\left(\widehat{u}_{0}\right), \kappa \neq 0$. Using the last equation to eliminate $k_{\kappa}^{-}\left(\widehat{u}_{0}\right)$, subtracting the second equation from the first, and dividing the second equation by $\|\kappa\|$, we are left with

$$
\|\kappa\|+2\left(\kappa /\|\kappa\| \mid k_{\kappa}^{+}\left(\widehat{u}_{0}\right)\right)=0, \quad\left(k_{\kappa}^{+}\left(\widehat{u}_{0}\right)\right)^{2}+\widehat{u}_{0}=0 .
$$

Hence, the square of $k_{\kappa}^{+}\left(\widehat{u}_{0}\right)$ is determined by $\widehat{u}_{0}$, while the Euclidean projection of $k_{\kappa}^{+}\left(\widehat{u}_{0}\right)$ onto the direction of $\kappa$ is determined by the length of $\kappa$. This allows for at most two solutions for $k_{\kappa}^{+}\left(\widehat{u}_{0}\right)$ and thus at most two solutions for the equations 4.4.3). Now, one solution of these equations maps into a different solution by $k_{\kappa}^{\mp}\left(\widehat{u}_{0}\right) \mapsto k_{-\kappa}^{ \pm}\left(\widehat{u}_{0}\right)$. Since $\Gamma^{*} \backslash\{0\}$ is invariant under the transformation $\kappa \mapsto-\kappa$ and the proposed solution in the statement of the theorem is indeed a solution by direct calculation, the entire set of solutions is captured (note that $\xi$ being the principal square root is an arbitrary choice because the symmetry operation mentioned above is precisely expressed by a sign change in $\xi$ ).

This theorem shows that the double points do not split for constant potential Fermi curves (recall that $k=0$ is not a double point of $F(0)$ in our sense). As is expected from corollary 4.3 .9 , the double points $k_{\kappa}^{\mp}\left(\widehat{u}_{0}\right)$ approach $k_{\kappa}^{\mp}$ for $\kappa$ far away from the origin because $\xi\left(\widehat{u}_{0}, \kappa\right)$ tends towards 1 in this case. This immediately yields the following generalisation of lemma 4.3.3.

Lemma 4.4.4. Let $4 \pi^{2} \widehat{u}_{0}$ be a constant potential and let $V \subseteq \mathbb{C}^{2} \backslash F\left(4 \pi^{2} \widehat{u}_{0}\right)$ be compact, then we have for lattice vectors $\rho, \nu \in \Gamma^{*}$ :

$$
\lim _{\|\nu\| \rightarrow \infty} \inf _{\substack{\rho \in \Gamma^{*} \\ k \in V}}\left|\left(\rho+k+k_{\nu}^{ \pm}\left(\widehat{u}_{0}\right)\right)^{2}+\widehat{u}_{0}\right|=\infty .
$$

More precisely, there is a $\delta>0$ such that

$$
(\nu, \rho) \mapsto \frac{1}{\left(\rho+k+k_{\nu}^{ \pm}\left(\widehat{u}_{0}\right)\right)^{2}+\widehat{u}_{0}}
$$

defines $c^{0}\left(\Gamma_{\delta}^{*}\right) \widehat{\otimes} \ell^{1, \infty}\left(\Gamma^{*}\right)$-functions holomorphically dependent on $k$ in the interior of $V$.

Proof. Expanding denominators yields

$$
\begin{aligned}
\left(\rho+k+k_{\nu}^{ \pm}\right)^{2} & =(\rho+k)^{2}+2\left(\rho+k \mid k_{\nu}^{ \pm}\right), \\
\left(\rho+k+k_{\nu}^{ \pm}\left(\widehat{u}_{0}\right)\right)^{2}+\widehat{u}_{0} & =(\rho+k)^{2}+2\left(\rho+k \mid k_{\nu}^{ \pm}\left(\widehat{u}_{0}\right)\right) .
\end{aligned}
$$


Since the $k_{\nu}^{\mp}\left(\widehat{u}_{0}\right)$ approach the $k_{\nu}^{\mp}$ as $\|\nu\| \rightarrow \infty$, lemma 4.3 .3 implies the first claim. The remainder follows as in the proof of lemma 4.3.3 except that we have to restrict ourselves to sufficiently large $\|\nu\|$ lest the denominator in 4.4.5 become zero.

\subsection{Asymptotic moduli parameterisation}

In sections 4.3 and 4.4 we proved that, asymptotically, a non-zero or a nonconstant potential, respectively, influences the Fermi curve at most near the handles. In this section, we shall investigate these handles, that is, the parts of the Fermi curve near a double point $\left(k_{\nu}^{-}, k_{\nu}^{+}\right)$(which is, for $\nu$ with sufficiently large norm, also near the double points $\left.\left(k_{\nu}^{-}\left(\widehat{u}_{0}\right), k_{\nu}^{+}\left(\widehat{u}_{0}\right)\right)\right)$ thoroughly.

\subsubsection{A nonlinear perturbation of the Fourier transform}

We first define a decomposition of certain Banach spaces.

Definition 4.5.1. For all $\nu \in \Gamma^{*}$, let $K_{\nu}$ be the complex Banach space generated by the Fourier modes $\psi_{0}$ and $\psi_{\nu}$. Unless $\nu=0, K_{\nu}$ is two-dimensional. Let us exclude the case $\nu=0$ from now on. Let $E$ be a Banach space of which $K_{\nu}$ is a closed subspace. Then the canonical projection

$$
\begin{aligned}
\pi_{K_{\nu}}: E & \rightarrow K_{\nu}, \\
f & \mapsto \widehat{f}(0) \psi_{0}+\widehat{f}(\nu) \psi_{\nu}
\end{aligned}
$$

is a bounded linear operator. In particular, this is the case for $E=\mathcal{F} \ell^{p, 1}\left(\Gamma^{*}\right)$, $1 \leq p \leq \infty$ and for $E=L^{2}(F)$. The projection $1_{E}-\pi_{K_{\nu}}$ is then bounded as well. We denote its image by $K_{\nu}^{\perp}$. Of course, $K_{\nu}^{\perp}$ depends on $E$ but we shall suppress this in notation as the implied space $E$ will be clear from context. These two projections induce a decomposition of $E$, which we will write as $E=K_{\nu} \oplus K_{\nu}^{\perp}$. It can be viewed as a generalisation of an orthogonal decomposition in a space with a scalar product (such as $\left.L^{2}(F)\right)$.

We shall also write $\widehat{\pi}_{K_{\nu}}$ for the analogous projection in the Fourier-transformed space, that is,

$$
\left(\widehat{\pi}_{K_{\nu}} \widehat{f}\right)(\kappa)=\widehat{\pi_{K_{\nu}} f}(\kappa)= \begin{cases}\widehat{f}(\kappa) & \text { if } \kappa \in\{0, \nu\} \\ 0 & \text { otherwise. }\end{cases}
$$

The relevance of the $K_{\nu}$ is that they are the kernels of the free Schrödinger operators at the double points:

Lemma 4.5.2. Let $\nu \in \Gamma^{*}, \nu \neq 0$. Then the kernel of $\Delta_{k_{\nu}^{ \pm}}$is $K_{ \pm \nu}$.

Proof. Lemma 4.2.2 and proposition 4.2.4 together with the relation $\nu=k_{\nu}^{-}-k_{\nu}^{+}$ imply

$$
\frac{1}{4} \Delta_{k_{\nu}^{-}}=\partial_{k_{\nu}^{-}} \bar{\partial}_{k_{\nu}^{-}}=\partial_{0} \bar{\partial}_{\nu}
$$

A Fourier transform of the free Schrödinger equation then yields (cf. 4.2.8)

$$
\left(i \kappa_{1}-\kappa_{2}\right)\left(i\left(\kappa_{1}+\nu_{1}\right)+\left(\kappa_{2}+\nu_{2}\right)\right) \widehat{\psi}(\kappa)=0 .
$$


This equation is fulfilled if and only if $\widehat{\psi}(\kappa)=0$ whenever $\kappa \neq 0$ and $\kappa \neq-\nu$. Hence the kernel of $\Delta_{k_{\nu}^{-}}$is $K_{-\nu}$. Analogously, one shows that the kernel of $\Delta_{k_{\nu}^{+}}$ is $K_{\nu}$.

This lemma shows that the singular support of the resolvent $\Delta_{k_{\nu}^{ \pm}}^{-1}$ is given by $K_{ \pm \nu}$, while on $K_{ \pm \nu}^{\perp}$ it is regular. Let us make these notions more precise.

Definition 4.5.3. Let $E_{1}, E_{2}$ be Banach spaces containing $K_{\nu}$ as closed subspaces, with decompositions $K_{\nu} \oplus K_{\nu}^{\perp}$ as described above, and let $T: E_{1} \rightarrow E_{2}$ be a linear operator. Then we can define the linear operators

$$
\begin{array}{ll}
A:=\left.\pi_{K_{\nu}} T\right|_{K_{\nu}}, & B:=\left.\pi_{K_{\nu}} T\right|_{K_{\nu}^{\perp}}, \\
C:=\left.\left(1-\pi_{K_{\nu}}\right) T\right|_{K_{\nu}}, & D:=\left.\left(1-\pi_{K_{\nu}}\right) T\right|_{K_{\nu}^{\perp}} .
\end{array}
$$

Hence, domains and codomains of these operators are given by

$$
\begin{array}{ll}
A: K_{\nu} \rightarrow K_{\nu}, & B: K_{\nu}^{\perp} \rightarrow K_{\nu}, \\
C: K_{\nu} \rightarrow K_{\nu}^{\perp}, & D: K_{\nu}^{\perp} \rightarrow K_{\nu}^{\perp} .
\end{array}
$$

Therefore, the operator $T$ has the matrix representation

$$
T=\left(\begin{array}{cc}
A & B \\
C & D
\end{array}\right)
$$

with respect to the decompositions $K_{\nu} \oplus K_{\nu}^{\perp}$. Since $K_{\nu}$ is closed, $A, B, C$ and $D$ are bounded if $T$ is bounded. We call the operator $A$ the restriction of $T$ to $K_{\nu}$ and, likewise, the operator $D$ the restriction of $T$ to $K_{\nu}^{\perp}$.

We can now show that the restriction of the free resolvent to $K_{ \pm \nu}^{\perp}$ is regular near $k_{\nu}^{ \pm}$.

Proposition 4.5.4. There is an open neighbourhood $V$ of $0 \in \mathbb{C}^{2}$ which depends only on $\Gamma^{*}$ and on which the free Schrödinger resolvent, either as an operator on $L^{2}(F)$ or as an operator $\mathcal{F} \ell^{\infty, 1}\left(\Gamma^{*}\right) \rightarrow \mathcal{F} \ell^{1}\left(\Gamma^{*}\right)$, has the $K_{\nu} \oplus K_{\nu}^{\perp}$ decomposition (according to definition 4.5.3)

$$
\Delta_{k_{\nu}^{ \pm}+k}^{-1}=\left(\begin{array}{cc}
S_{ \pm \nu}\left(k_{\nu}^{ \pm}+k\right) & 0 \\
0 & R_{ \pm \nu}\left(k_{\nu}^{ \pm}+k\right)
\end{array}\right)
$$

for all $\nu \in \Gamma^{*}, \nu \neq 0$, where $R_{ \pm \nu}\left(k_{\nu}^{ \pm}+k\right)$ is holomorphic for $k \in V$, in particular,

$$
\sup _{k \in V} \sup _{\nu \in \Gamma^{*} \backslash\{0\}}\left\|R_{ \pm \nu}\left(k+k_{\nu}^{ \pm}\right)\right\|<\infty
$$

where $\|\cdot\|$ denotes the respective operator norm, and $S_{ \pm \nu}$ is given by

$$
S_{ \pm \nu}(k)=\left(\begin{array}{cc}
-4 \pi^{2} k^{2} & 0 \\
0 & -4 \pi^{2}(k \pm \nu)^{2}
\end{array}\right)^{-1}
$$

with respect to the basis $\left(\psi_{0}, \psi_{ \pm \nu}\right)$. 
Proof. Let $k_{0} \in \mathbb{C}^{2}$ be a pole of $k \mapsto \Delta_{k}^{-1}$. Then by theorem 4.2.5 there is a $\kappa \in \Gamma^{*}$ such that one of the following equations holds:

$$
k_{0,2}+\kappa_{2}=i\left(k_{0,1}+\kappa_{1}\right), \quad k_{0,2}+\kappa_{2}=-i\left(k_{0,1}+\kappa_{1}\right) .
$$

If $k_{0}=k_{\nu}^{ \pm}$, then this can only happen if $\kappa=0$ or $\kappa= \pm \nu$ by lemma 4.5.2 Therefore, if $k_{0}=k+k_{\nu}^{ \pm}$where the norm of $k$ is sufficiently small (say, smaller than half of the minimum distance of distinct vectors in $\Gamma_{\mathbb{C}}^{*}$ ), this can also only happen for the same $\kappa$ because $\kappa_{1}, \kappa_{2} \in \mathbb{R}$ and $\Gamma^{*}$ is discrete. Hence, all possible poles are contained in the restriction $S_{ \pm \nu}$ of $\Delta_{k}^{-1}$ to $K_{ \pm \nu}$ in this case, while $R_{ \pm \nu}$, the restriction of $\Delta_{k}^{-1}$ to $K_{ \pm \nu}^{\perp}$, is pole-free in a small neighbourhood of $k_{\nu}^{ \pm}$. The estimate on the norm follows in similar fashion as in the proof of theorem 3.2.5. We can calculate $S_{ \pm \nu}$ explicitly in the usual way by investigating the Fourier transform, to wit

$$
\begin{gathered}
\widehat{\psi}_{0} \mapsto \frac{1}{-4 \pi^{2}\left(k+k_{\nu}^{ \pm}\right)^{2}} \widehat{\psi}_{0}, \\
\widehat{\psi}_{ \pm \nu} \mapsto \frac{1}{-4 \pi^{2}\left(k+k_{\nu}^{ \pm} \pm \nu\right)^{2}} \widehat{\psi}_{ \pm \nu} .
\end{gathered}
$$

This proves the claims.

For $\nu \in \Gamma^{*}$ with sufficiently large norm, this proposition extends to constant potentials.

Proposition 4.5.6. There is an open neighbourhood $V$ of $0 \in \mathbb{C}^{2}$ which depends only on $\Gamma^{*}$ such that for all constant potentials $4 \pi^{2} \widehat{u}_{0}$ there is a $\delta>0$ such that the Schrödinger resolvent for $4 \pi^{2} \widehat{u}_{0}$, either as an operator on $L^{2}(F)$ or as an operator $\mathcal{F} \ell^{\infty, 1}\left(\Gamma^{*}\right) \rightarrow \mathcal{F} \ell^{1}\left(\Gamma^{*}\right)$, has the $K_{\nu} \oplus K_{\nu}^{\perp}$-decomposition (according to definition 4.5.3.

$$
\left(\Delta_{k_{\nu}^{ \pm}\left(\widehat{u}_{0}\right)+k}-4 \pi^{2} \widehat{u}_{0}\right)^{-1}=\left(\begin{array}{cc}
S_{ \pm \nu}\left(k_{\nu}^{ \pm}\left(\widehat{u}_{0}\right)+k, \widehat{u}_{0}\right) & 0 \\
0 & R_{ \pm \nu}\left(k_{\nu}^{ \pm}\left(\widehat{u}_{0}\right)+k, \widehat{u}_{0}\right)
\end{array}\right)
$$

for all $\nu \in \Gamma_{\delta}^{*}$, where $R_{ \pm \nu}\left(k_{\nu}^{ \pm}\left(\widehat{u}_{0}\right)+k, \widehat{u}_{0}\right)$ is holomorphic for $k \in V$, in particular,

$$
\sup _{k \in V} \sup _{\nu \in \Gamma_{\delta}^{*}}\left\|R_{ \pm \nu}\left(k+k_{\nu}^{ \pm}\left(\widehat{u}_{0}\right), \widehat{u}_{0}\right)\right\|<\infty,
$$

where $\|\cdot\|$ denotes the respective operator norm, and $S_{ \pm \nu}\left(\cdot, \widehat{u}_{0}\right)$ is given by

$$
S_{ \pm \nu}\left(k, \widehat{u}_{0}\right)=\left(\begin{array}{cc}
-4 \pi^{2}\left(k^{2}+\widehat{u}_{0}\right) & 0 \\
0 & -4 \pi^{2}\left((k \pm \nu)^{2}+\widehat{u}_{0}\right)
\end{array}\right)^{-1}
$$

with respect to the basis $\left(\psi_{0}, \psi_{ \pm \nu}\right)$.

Proof. Since $4 \pi^{2} \widehat{u}_{0}$ is constant, the decomposition of $\left(\Delta_{k_{\nu}^{ \pm}\left(\widehat{u}_{0}\right)+k}-4 \pi^{2} \widehat{u}_{0}\right)^{-1}$ is diagonal. By theorem 4.4.1, the equation

$$
\left(k_{\nu}^{ \pm}\left(\widehat{u}_{0}\right)+\kappa\right)^{2}+\widehat{u}_{0}=0
$$

is solved by $\kappa=0$ and $\kappa= \pm \nu$. Furthermore, $k_{\nu}^{ \pm}\left(\widehat{u}_{0}\right)$ approaches $k_{\nu}^{ \pm}$as $\|\nu\| \rightarrow$ $\infty$. Hence, the claim follows from proposition 4.5.4. 
It may have occurred to the reader that the kernel of $\Delta_{k}$ at $k_{\nu}^{-}$equals the kernel at $k_{-\nu}^{+}$, namely $K_{-\nu}$, and the kernel at $k_{\nu}^{+}$equals the kernel at $k_{-\nu}^{-}$, namely $K_{\nu}$. Likewise, for constant potential resolvents and sufficiently large $\|\nu\|$ in dependence of the potential, the singularities both near $k=k_{\nu}^{-}\left(\widehat{u}_{0}\right)$ and near $k=k_{-\nu}^{+}\left(\widehat{u}_{0}\right)$ are contained in the restriction to $K_{-\nu}$ and the singularities both near $k=k_{\nu}^{+}\left(\widehat{u}_{0}\right)$ and near $k=k_{-\nu}^{-}\left(\widehat{u}_{0}\right)$ are contained in the restriction to $K_{\nu}$. On the other hand, at the two components $k_{\nu}^{-}\left(\widehat{u}_{0}\right)$ and $k_{\nu}^{+}\left(\widehat{u}_{0}\right)$ of a double point, the respective spaces $K_{-\nu}$ and $K_{\nu}$ are different. This latter fact is not a surprise by now, as the difference $k_{\nu}^{-}\left(\widehat{u}_{0}\right)-k_{\nu}^{+}\left(\widehat{u}_{0}\right)=\nu$ produces a shift by $\nu$ in the Fourier transform of the Schrödinger operator, so that we get the map

$$
K_{-\nu} \rightarrow K_{\nu}, \quad \psi_{-\nu} \mapsto \psi_{0}, \quad \psi_{0} \mapsto \psi_{\nu} .
$$

This also explains the former behaviour because $k_{-\nu}^{ \pm}\left(\widehat{u}_{0}\right)=-k_{\nu}^{ \pm}\left(\widehat{u}_{0}\right)$, Fermi curves are invariant under shifts by vectors from $\Gamma^{*}$, and we also have the identity $F\left(4 \pi^{2} \widehat{u}_{0}\right)=-F\left(4 \pi^{2} \widehat{u}_{0}\right)$. Interestingly, this latter property is shared by all Fermi curves:

Proposition 4.5.8. Let $u \in \mathcal{F} \ell^{\infty, 1}\left(\Gamma^{*}\right)$. Then $F(u)=-F(u)$.

Proof. We may consider the Schrödinger operator $-\Delta_{k}+u$ a closed operator on $L^{2}(F)$. By considering the canonical Euclidean bilinear form on $L^{2}(F)$ (not the standard Hermitian form!), we may talk of the transpose of the Schrödinger operator. Clearly, the transposed Schrödinger operator produces the same Fermi curve as the untransposed one. Now, a partial integration (recall that we have periodic boundary conditions) shows that for the Wirtinger operators, we have

$$
\partial^{t}=-\partial, \quad \bar{\partial}^{t}=-\bar{\partial} .
$$

The transpose of multiplication operators remains invariant, so

$$
\partial_{k}^{t}=-\partial_{-k}, \quad \bar{\partial}_{k}^{t}=-\bar{\partial}_{-k},
$$

and, of course, $u^{t}=u$. By lemma 4.2.2 we therefore have

$$
\left(-\Delta_{k}+u\right)^{t}=-\Delta_{-k}+u
$$

proving the proposition.

Note that this proposition is true for $d>2$ as well, but it does not extend to the Bloch varieties because the transpose leaves the eigenvalue invariant. In proposition 4.5.4 and proposition 4.5.6. we can take $V$ to be point-symmetric, that is, $V=-V$, for example when $V$ is a small open ball. Hence we can identify the Fermi curve around $k_{\nu}^{+}\left(\widehat{u}_{0}\right)$ with the Fermi curve around $k_{-\nu}^{+}\left(\widehat{u}_{0}\right)$ by a sign change and thus work on a double point with a single decomposition $K_{\nu} \oplus K_{\nu}^{\perp}$.

Next, we shall want to see what happens asymptotically to the fact implied by proposition 4.5.4 and proposition 4.5.6. namely that the restriction of the free Schrödinger resolvent to $K_{ \pm \nu}^{\perp}$ is regular around $k_{\nu}^{ \pm}\left(\widehat{u}_{0}\right)$, when we add a non-constant potential. To this end, we first prove an analogon of lemma 4.3 .3 and lemma 4.4.4 
Lemma 4.5.9. There is an open neighbourhood $V$ of $0 \in \mathbb{C}^{2}$ which depends only on $\Gamma^{*}$ such that

$$
\lim _{\|\nu\| \rightarrow \infty} \inf _{\substack{k \in V \\ \rho \in \Gamma^{*} \\ \rho \neq 0, \pm \nu}}\left|\left(\rho+k+k_{\nu}^{ \pm}\right)^{2}\right|=\infty .
$$

More precisely,

$$
(\nu, \rho) \mapsto \begin{cases}\frac{1}{\left(\rho+k+k_{\nu}^{ \pm}\right)^{2}}, & \rho \neq 0, \pm \nu \\ 0 & \text { otherwise }\end{cases}
$$

defines $c^{0}\left(\Gamma^{*}\right) \widehat{\otimes} \ell^{1, \infty}\left(\Gamma^{*}\right)$-functions holomorphically dependent on $k$.

Likewise, if $4 \pi^{2} \widehat{u}_{0}$ is a constant potential, then

$$
\lim _{\|\nu\| \rightarrow \infty} \inf _{\substack{k \in V \\ \rho \in \Gamma^{*} \\ \rho \neq 0, \pm \nu}}\left|\left(\rho+k+k_{\nu}^{ \pm}\left(\widehat{u}_{0}\right)\right)^{2}+\widehat{u}_{0}\right|=\infty,
$$

and there is a $\delta>0$ such that

$$
(\nu, \rho) \mapsto \begin{cases}\frac{1}{\left(\rho+k+k_{\nu}^{ \pm}\left(\widehat{u}_{0}\right)\right)^{2}+\widehat{u}_{0}}, & \rho \neq 0, \pm \nu \\ 0 & \text { otherwise }\end{cases}
$$

defines $c^{0}\left(\Gamma_{\delta}^{*}\right) \widehat{\otimes} \ell^{1, \infty}\left(\Gamma^{*}\right)$-functions holomorphically dependent on $k$.

Proof. The proof is essentially analogous to the proof of lemma 4.3.3. This time, the respective factors in the equation analogous to equation (4.3.5) are bounded away from zero for a sufficiently small neighbourhood $V$ because the zeros of $\left(\rho+k_{\nu}^{ \pm}\right)^{2}$ are precisely $\rho=0$ and $\rho= \pm \nu$, and those are excluded from the infimum. For non-zero constant potentials, this follows from lemma 4.4.4 in the same way.

An analogon to lemma 4.3 .7 is next.

Lemma 4.5.12. There is an open neighbourhood $V$ of $0 \in \mathbb{C}^{2}$ which depends only on $\Gamma^{*}$ such that for all $u_{0} \in \mathcal{F} \ell^{\infty, 1}\left(\Gamma^{*}\right)$ and all sufficiently small $\epsilon>0$ there is an $N \in \mathbb{N}$ and $a C>0$ with $u_{0} \in B_{C}^{N}$ as well as a $\delta>0$ such that for all $k \in V$, all $\nu \in \Gamma_{\delta}^{*}$ and all $u \in B_{C}^{N}$ the restriction of the operator $\Delta_{k_{\nu}^{ \pm}+k}^{-1} u$ to $K_{\nu}^{\perp} \subseteq \mathcal{F} \ell^{1}\left(\Gamma^{*}\right)$ is bounded with

$$
\left\|\Delta_{k_{\nu}^{ \pm}+k}^{-1} u\right\|<\epsilon .
$$

Proof. The proof is similar to the proof of lemma 4.3.7. Nevertheless, we shall give a full proof because the order of operators has been reversed.

By proposition 2.4.15. multiplication with any $u \in \mathcal{F} \ell^{\infty, 1}\left(\Gamma^{*}\right)$ maps $\mathcal{F} \ell^{1}\left(\Gamma^{*}\right)$ boundedly into $\mathcal{F} \ell^{\infty, 1}\left(\Gamma^{*}\right)$. By proposition 4.5.4.

$$
\left.\left(1-\pi_{K_{ \pm \nu}}\right) \Delta_{k+k_{\nu}^{ \pm}}^{-1} u\right|_{K_{ \pm \nu}^{\perp}}=\left.\Delta_{k+k_{\nu}^{ \pm}}^{-1}\left(1-\pi_{K_{ \pm \nu}}\right) u\right|_{K_{ \pm \nu}^{\perp}},
$$

and hence the restriction of $\Delta_{k+k_{\nu}^{ \pm}}^{-1} u$ to $K_{ \pm \nu}^{\perp} \subseteq \mathcal{F} \ell^{1}\left(\Gamma^{*}\right)$ is bounded for all $k \in V$ with a suitable $V$. Let us now estimate the norm of this operator. Set

$$
C:=\frac{\epsilon \mu(F)}{2 \sup _{k \in V} \sup _{\nu \in \Gamma^{*} \backslash\{0\}}\left\|R_{ \pm \nu}\left(k+k_{\nu}^{ \pm}\right)\right\|}>0 .
$$


By proposition 2.8.18, there is an $N \in \mathbb{N}$ such that

$$
\left\|\widehat{u}_{0}\right\|_{(\infty, 1) ; N}<C .
$$

By definition, this inequality then holds for all $u \in B_{C}^{N}$. For each such $u$ let $A_{u} \subseteq \Gamma^{*}$ with $\mu\left(A_{u}\right)=N$ such that the $N$-localised quasi-norm of $u$ equals the $A_{u}$-localised norm and set

$$
c:=\sup _{u \in B_{C}^{N}}\left\|\widehat{u}-\widehat{u}_{A_{u}}\right\|_{\infty}<\infty .
$$

We decompose each $u \in B_{C}^{N}$ into $u_{1}$ and $u_{2}$ such that

$$
\widehat{u}_{1}:=\widehat{u}_{A_{u}}, \quad \widehat{u}_{2}:=\widehat{u}-\widehat{u}_{A_{u}} .
$$

Let $f \in \mathcal{F} \ell^{1}\left(\Gamma^{*}\right)$, then

$$
\left\|\Delta_{k+k_{\nu}^{ \pm}}^{-1}\left(1-\pi_{K_{\nu}}\right) u_{1} f\right\|_{\mathcal{F} 1}<\frac{1}{2}\|f\|_{\mathcal{F} 1} .
$$

To obtain the analogous estimate for $u_{2}$, we note that

$$
\left|\frac{1}{4 \pi^{2}\left(k+k_{\nu}^{ \pm}+\kappa\right)^{2}} \sum_{\rho \in \Gamma^{*}} \widehat{u}_{2}(\rho) \widehat{f}(\kappa-\rho)\right| \leq \frac{c}{4 \pi^{2}\left|\left(k+k_{\nu}^{ \pm}+\kappa\right)^{2}\right|} \sum_{\rho \in A_{u}}|\widehat{f}(\kappa-\rho)| .
$$

Then, for a suitable levelling operator $\Lambda$ :

$$
\sum_{\substack{\kappa \in \Gamma^{*} \\ \kappa \neq 0, \pm \nu}} \frac{c}{4 \pi^{2}\left|\left(k+k_{\nu}^{ \pm}+\kappa\right)^{2}\right|} \sum_{\rho \in A_{u}}|\widehat{f}(\kappa-\rho)| \leq \frac{c N}{\inf _{\substack{k \in V^{*} \\ \kappa \in \Gamma^{*} \\ \kappa \neq 0, \pm \nu}}\left|\left(k+k_{\nu}^{ \pm}+\kappa\right)^{2}\right|}\|\Lambda|\widehat{f}|\|_{1} .
$$

The claim now follows from lemma 4.5.9.

This lemma is of the same type than lemma 4.3 .7 and also lemmata 3.3 .4 and 3.3.6. With the "extended" boundary conditions introduced in section 4.4. we can prove a variation of this lemma where we compose the constant potential resolvent with a zero-average potential. This last restriction is, at this point, not strictly necessary. But it will be required later, and is of course the whole point of section 4.4 in the first place.

Lemma 4.5.13. There is an open neighbourhood of $0 \in \mathbb{C}^{2}$ which depends only on $\Gamma^{*}$ such that for all $u_{0} \in \mathcal{F} \ell^{\infty, 1}\left(\Gamma^{*}\right)$ and all sufficiently small $\epsilon>0$ there is an $N \in \mathbb{N}$ and a $C>0$ with $u_{0} \in B_{C}^{N}$ as well as a $\delta>0$ such that for all $k \in V$, all $\nu \in \Gamma_{\delta}^{*}$ and all $u \in B_{C}^{N}$ the restriction of the operator $\left(\Delta_{k_{\nu}^{ \pm}\left(\widehat{u}_{0}\right)+k}-\right.$ $\left.4 \pi^{2} \widehat{u}_{0}\right)^{-1} \bar{u}$, where $4 \pi^{2} \widehat{u}_{0}$ is the constant part and $\bar{u}$ is the zero-average part of $u$, to $K_{\nu}^{\perp} \subseteq \mathcal{F} \ell^{1}\left(\Gamma^{*}\right)$ is bounded with

$$
\left\|\left(\Delta_{k_{\nu}^{ \pm}\left(\widehat{u}_{0}\right)+k}-4 \pi^{2} \widehat{u}_{0}\right)^{-1} \bar{u}\right\|<\epsilon .
$$

Proof. The proof is analogous to the proof of lemma 4.3.7. We merely need to use $\left(\Delta_{k+k_{\nu}^{ \pm}\left(\widehat{u}_{0}\right)}-4 \pi^{2} \widehat{u}_{0}\right)^{-1}, \bar{u}$ and $R_{ \pm \nu}\left(k+k_{\nu}^{ \pm}\left(\widehat{u}_{0}\right), \widehat{u}_{0}\right)$ in place of $\Delta_{k+k_{\nu}^{ \pm}}^{-1}, u$ and $R_{ \pm}\left(k+k_{\nu}^{ \pm}\right)$, respectively, and use the second half of lemma 4.5.9 instead of the first one. 
Incidentally, the proof of lemma 4.5 .12 and lemma 4.5 .13 works not only for $\left.\left(1-\pi_{K_{ \pm \nu}}\right) \Delta_{k+k_{\nu}^{ \pm}}^{-1} u\right|_{K_{ \pm \nu}}$ and $\left.\left(1-\pi_{K_{ \pm \nu}}\right)\left(\Delta_{k+k_{\nu}^{ \pm}\left(\widehat{u}_{0}\right)}-4 \pi^{2} \widehat{u}_{0}\right)^{-1} \bar{u}\right|_{K_{ \pm \nu}}$ but also for $\left(1-\pi_{K_{ \pm \nu}}\right) \Delta_{k+k_{\nu}^{ \pm}}^{-1} u$ and $\left(1-\pi_{K_{ \pm \nu}}\right)\left(\Delta_{k+k_{\nu}^{ \pm}\left(\widehat{u}_{0}\right)}-4 \pi^{2} \widehat{u}_{0}\right)^{-1} \bar{u}$, allowing for the application of the Neumann theorem on both $K_{ \pm \nu}^{\perp}$ and $\mathcal{F} \ell^{1}\left(\Gamma^{*}\right)$. We generalise this simple principle with the following lemma.

Lemma 4.5.14. Let $E$ be a Banach space, $E^{\prime}$ a closed subspace of $E$ and $T: E \rightarrow E^{\prime}$ a bounded linear operator. Let $1_{E}$ and $1_{E^{\prime}}$ denote the identity operators on $E$ and $E^{\prime}$, respectively. Then $1_{E^{\prime}}-\left.T\right|_{E^{\prime}}$ is boundedly invertible on $E^{\prime}$ if and only if $1_{E}-T$ is boundedly invertible on $E$.

Proof. Assume first that $1_{E}-T$ is boundedly invertible. Since the codomain of $T$ is $E^{\prime}$, we have $\left(1_{E}-T\right) E^{\prime} \subseteq E^{\prime}$ and $\left(1_{E}-T\right)\left(E \backslash E^{\prime}\right) \subseteq E \backslash E^{\prime}$. These inclusions become equalities because $1_{E}-T$ is invertible. Hence, the inverse of $1_{E^{\prime}}-\left.T\right|_{E^{\prime}}$ exists and is given by the restriction of $\left(1_{E}-T\right)^{-1}$ to $E^{\prime}$. Boundedness follows from the boundedness of $\left(1_{E}-T\right)^{-1}$.

Now assume $1_{E^{\prime}}-\left.T\right|_{E^{\prime}}$ to be boundedly invertible. Since $\left(1_{E}-T\right) E^{\prime} \subseteq E^{\prime}$, we have

$$
\left(1_{E}-T\right)\left(1_{E^{\prime}}-\left.T\right|_{E^{\prime}}\right)^{-1}=1_{E^{\prime}}
$$

and

$$
T\left(1_{E}-T\right)=T 1_{E}-T T=1_{E^{\prime}} T-\left.T\right|_{E^{\prime}} T=\left(1_{E^{\prime}}-\left.T\right|_{E^{\prime}}\right) T .
$$

Therefore,

$$
\left(1_{E}-T\right)\left(1_{E}+\left(1_{E^{\prime}}-\left.T\right|_{E^{\prime}}\right)^{-1} T\right)=1_{E}-T+1_{E^{\prime}} T=1_{E}
$$

and

$$
\begin{aligned}
& \left(1_{E}+\left(1_{E^{\prime}}-\left.T\right|_{E^{\prime}}\right)^{-1} T\right)\left(1_{E}-T\right) \\
& \quad=1_{E}-T+\left(1_{E^{\prime}}-\left.T\right|_{E^{\prime}}\right)^{-1}\left(1_{E^{\prime}}-\left.T\right|_{E^{\prime}}\right) T=1_{E}-T+T=1_{E} .
\end{aligned}
$$

Hence, the inverse $\left(1_{E}-T\right)^{-1}$ exists and is given by $1_{E}+\left(1_{E^{\prime}}-\left.T\right|_{E^{\prime}}\right)^{-1} T$. Since both $T$ and $\left(1_{E^{\prime}}-\left.T\right|_{E^{\prime}}\right)^{-1}$ are bounded, the inverse is bounded as well.

We can now begin to develop a criterion determining when $k \in F(u)$ for $k$ near a double point $k_{\nu}^{ \pm}\left(\widehat{u}_{0}\right)$ with $\|\nu\|$ sufficiently large. Recall the definition 3.3 .2 of the open sets $B_{C}^{N}$.

Proposition 4.5.15. There is an open neighbourhood $V$ of $0 \in \mathbb{C}^{2}$ which depends only on $\Gamma^{*}$ such that for all $u_{0} \in \mathcal{F} \ell^{\infty, 1}\left(\Gamma^{*}\right)$ there is an $N \in \mathbb{N}$ and a $C>0$ with $u_{0} \in B_{C}^{N}$ as well as a $\delta>0$ such that for all $k \in V$, all $\nu \in \Gamma_{\delta}^{*}$ and all $u \in B_{C}^{N}$ the restriction of the operator

$$
\bar{u}\left(1-\left(1-\pi_{K_{ \pm \nu}}\right)\left(\Delta_{k+k_{\nu}^{ \pm}\left(\widehat{u}_{0}\right)}-4 \pi^{2} \widehat{u}_{0}\right)^{-1} \bar{u}\right)^{-1},
$$

where $4 \pi^{2} \widehat{u}_{0}$ is the constant part and $\bar{u}$ is the zero-average part of $u$, to $K_{ \pm \nu}$ exists and is bounded. The same holds for the operator

$$
\left(\Delta_{k+k_{\nu}^{ \pm}\left(\widehat{u}_{0}\right)}-4 \pi^{2} \widehat{u}_{0}\right)^{-1} \bar{u}\left(1-\left(1-\pi_{K_{ \pm \nu}}\right)\left(\Delta_{k+k_{\nu}^{ \pm}\left(\widehat{u}_{0}\right)}-4 \pi^{2} \widehat{u}_{0}\right)^{-1} \bar{u}\right)^{-1}
$$


whenever $k+k_{\nu}^{ \pm}\left(\widehat{u}_{0}\right) \notin F\left(4 \pi^{2} \widehat{u}_{0}\right){ }^{5}$. Furthermore, those $k \in V \cap\left(F(u)-k_{\nu}^{ \pm}\left(\widehat{u}_{0}\right)\right)$ for which $k \notin F\left(4 \pi^{2} \widehat{u}_{0}\right)$, are characterised by the poles of

$$
\begin{aligned}
k+k_{\nu}^{ \pm}\left(\widehat{u}_{0}\right) \mapsto & \left(1_{K_{ \pm \nu}}-\pi_{K_{ \pm \nu}}\left(\Delta_{k+k_{\nu}^{ \pm}\left(\widehat{u}_{0}\right)}-4 \pi^{2} \widehat{u}_{0}\right)^{-1} \bar{u}\right. \\
& \left.\left.\left(1-\left(1-\pi_{K_{ \pm \nu}}\right)\left(\Delta_{k+k_{\nu}^{ \pm}\left(\widehat{u}_{0}\right)}-4 \pi^{2} \widehat{u}_{0}\right)^{-1} \bar{u}\right)^{-1}\right|_{K_{ \pm \nu}}\right)^{-1}
\end{aligned}
$$

Proof. By lemma 4.5.13 for all $\epsilon>0$ there are appropriate $V, N, C, \delta$, such that for all $k \in V, u \in B_{C}^{N}, \nu \in \Gamma_{\delta}^{*}$, the restriction of the operator $\left(\Delta_{k_{\nu}^{ \pm}\left(\widehat{u}_{0}\right)+k}-\right.$ $\left.4 \pi^{2} \widehat{u}_{0}\right)^{-1} \bar{u}$ is bounded by $\epsilon>0$. For sufficiently small $\epsilon>0$, the Neumann theorem implies that

$$
1_{K_{ \pm \nu}^{\perp}}-\left.\left(1-\pi_{K_{ \pm \nu}}\right)\left(\Delta_{k_{\nu}^{ \pm}\left(\widehat{u}_{0}\right)+k}-4 \pi^{2} \widehat{u}_{0}\right)^{-1} \bar{u}\right|_{K_{ \pm \nu}^{\perp}}
$$

is invertible. Hence, by lemma 4.5 .14 , the operator

$$
1-\left(1-\pi_{K_{ \pm \nu}}\right)\left(\Delta_{k_{\nu}^{ \pm}\left(\widehat{u}_{0}\right)+k}-4 \pi^{2} \widehat{u}_{0}\right)^{-1} \bar{u}
$$

is invertible on $\mathcal{F} \ell^{1}\left(\Gamma^{*}\right)$. Therefore, the operator

$$
\bar{u}\left(1-\left(1-\pi_{K_{ \pm \nu}}\right)\left(\Delta_{k_{\nu}^{ \pm}\left(\widehat{u}_{0}\right)+k}-4 \pi^{2} \widehat{u}_{0}\right)^{-1} \bar{u}\right)^{-1}
$$

exists and is bounded from $\mathcal{F} \ell^{1}\left(\Gamma^{*}\right)$ to $\mathcal{F} \ell^{\infty, 1}\left(\Gamma^{*}\right)$. This also holds for the restriction to $K_{ \pm \nu}$. By proposition 4.5.6, the same holds for the operator

$$
\left(\Delta_{k+k_{\nu}^{ \pm}\left(\widehat{u}_{0}\right)}-4 \pi^{2} \widehat{u}_{0}\right)^{-1} \bar{u}\left(1-\left(1-\pi_{K_{ \pm \nu}}\right)\left(\Delta_{k_{\nu}^{ \pm}\left(\widehat{u}_{0}\right)+k}-4 \pi^{2} \widehat{u}_{0}\right)^{-1} \bar{u}\right)^{-1}
$$

on $\mathcal{F} \ell^{1}\left(\Gamma^{*}\right)$ whenever $k+k_{\nu}^{ \pm}\left(\widehat{u}_{0}\right) \notin F\left(4 \pi^{2} \widehat{u}_{0}\right)$. By lemma 4.5.14, the map 4.5.16 has a pole at $k_{0}+k_{\nu}^{ \pm}\left(\widehat{u}_{0}\right)$ if and only if

$$
\begin{aligned}
k+k_{\nu}^{ \pm}\left(\widehat{u}_{0}\right) \mapsto\left(1-\pi_{K_{ \pm \nu}}\left(\Delta_{k+k_{\nu}^{ \pm}\left(\widehat{u}_{0}\right)}-4 \pi^{2} \widehat{u}_{0}\right)^{-1} \bar{u}\right. & \\
& \left.\left(1-\left(1-\pi_{K_{ \pm \nu}}\right)\left(\Delta_{k_{\nu}^{ \pm}\left(\widehat{u}_{0}\right)+k}-4 \pi^{2} \widehat{u}_{0}\right)^{-1} \bar{u}\right)^{-1}\right)^{-1}
\end{aligned}
$$

has a pole there. This expression, in turn, has a pole at $k_{0}+k_{\nu}^{ \pm}\left(\widehat{u}_{0}\right)$ if and only if

$$
\begin{aligned}
& k+k_{\nu}^{ \pm}\left(\widehat{u}_{0}\right) \mapsto(1-\left.\left(1-\pi_{K_{ \pm \nu}}\right)\left(\Delta_{k+k_{\nu}^{ \pm}\left(\widehat{u}_{0}\right)}-4 \pi^{2} \widehat{u}_{0}\right)^{-1} \bar{u}\right)^{-1} \\
&\left(1-\pi_{K_{ \pm \nu}}\left(\Delta_{k+k_{\nu}^{ \pm}\left(\widehat{u}_{0}\right)}-4 \pi^{2} \widehat{u}_{0}\right)^{-1} \bar{u}\right. \\
&\left.\left(1-\left(1-\pi_{K_{ \pm}}\right)\left(\Delta_{k+k_{\nu}^{ \pm}\left(\widehat{u}_{0}\right)}-4 \pi^{2} \widehat{u}_{0}\right)^{-1} \bar{u}\right)^{-1}\right)^{-1} \\
&\left(\Delta_{k+k_{\nu}^{ \pm}\left(\widehat{u}_{0}\right)}-4 \pi^{2} \widehat{u}_{0}\right)^{-1}
\end{aligned}
$$

\footnotetext{
${ }^{5}$ In the first case, the operator is the restriction of an operator from $\mathcal{F} \ell^{1}\left(\Gamma^{*}\right)$ to $\mathcal{F} \ell^{\infty, 1}\left(\Gamma^{*}\right)$. In the second case, the operator is the restriction of an operator on $\mathcal{F} \ell^{1}\left(\Gamma^{*}\right)$.
} 
has a pole there. Now,

$$
\begin{aligned}
& \left(1-\left(1-\pi_{K_{ \pm \nu}}\right)\left(\Delta_{k+k_{\nu}^{ \pm}\left(\widehat{u}_{0}\right)}-4 \pi^{2} \widehat{u}_{0}\right)^{-1} \bar{u}\right)^{-1} \\
& \left(1-\pi_{K_{ \pm \nu}}\left(\Delta_{k+k_{\nu}^{ \pm}\left(\widehat{u}_{0}\right)}-4 \pi^{2} \widehat{u}_{0}\right)^{-1} \bar{u}\right. \\
& \left.\left(1-\left(1-\pi_{K_{ \pm \nu}}\right)\left(\Delta_{k+k_{\nu}^{ \pm}\left(\widehat{u}_{0}\right)}-4 \pi^{2} \widehat{u}_{0}\right)^{-1} \bar{u}\right)^{-1}\right)^{-1} \\
& \left(\Delta_{k+k_{\nu}^{ \pm}\left(\widehat{u}_{0}\right)}-4 \pi^{2} \widehat{u}_{0}\right)^{-1} \\
= & \left(1-\left(1-\pi_{K_{ \pm \nu}}\right)\left(\Delta_{k+k_{\nu}^{ \pm}\left(\widehat{u}_{0}\right)}-4 \pi^{2} \widehat{u}_{0}\right)^{-1} \bar{u}\right. \\
& \left.\quad-\pi_{K_{ \pm \nu}}\left(\Delta_{k+k_{\nu}^{ \pm}\left(\widehat{u}_{0}\right)}-4 \pi^{2} \widehat{u}_{0}\right)^{-1} \bar{u}\right)^{-1}\left(\Delta_{k+k_{\nu}^{ \pm}\left(\widehat{u}_{0}\right)}-4 \pi^{2} \widehat{u}_{0}\right)^{-1} \\
= & \left(1-\left(\Delta_{k+k_{\nu}^{ \pm}\left(\widehat{u}_{0}\right)}-4 \pi^{2} \widehat{u}_{0}\right)^{-1} \bar{u}\right)^{-1}\left(\Delta_{k+k_{\nu}^{ \pm}\left(\widehat{u}_{0}\right)}-4 \pi^{2} \widehat{u}_{0}\right)^{-1} \\
= & \left(\Delta_{k+k_{\nu}^{ \pm}\left(\widehat{u}_{0}\right)}-u\right)^{-1},
\end{aligned}
$$

proving the proposition.

This proposition shows that asymptotically near the double points, the part of $F(u)$ that does not belong to the constant-potential Fermi curve is described by the poles of an operator-valued function on a but two-dimensional space. Therefore, we make the following definition, from now on consistently denoting the constant and the zero-average part of some potential $u$ by $4 \pi^{2} \widehat{u}_{0}$ and $\bar{u}$, respectively.

Definition 4.5.18. For all $k \in \mathbb{C}^{2}$, all $u \in \mathcal{F} \ell^{\infty, 1}\left(\Gamma^{*}\right)$ and all $\nu \in \Gamma^{*} \backslash\{0\}$ such that the operator

$$
1-\left(1-\pi_{K_{ \pm \nu}}\right)\left(\Delta_{k+k_{\nu}^{ \pm}\left(\widehat{u}_{0}\right)}-4 \pi^{2} \widehat{u}_{0}\right)^{-1} \bar{u}
$$

exists and is boundedly invertible on $\mathcal{F} \ell^{1}\left(\Gamma^{*}\right)$, let $\mathcal{A}_{ \pm \nu}\left(k+k_{\nu}^{ \pm}\left(\widehat{u}_{0}\right), u\right)$ be the restriction of the operator

$$
\bar{u}\left(1-\left(1-\pi_{K_{ \pm \nu}}\right)\left(\Delta_{k+k_{\nu}^{ \pm}\left(\widehat{u}_{0}\right)}-4 \pi^{2} \widehat{u}_{0}\right)^{-1} \bar{u}\right)^{-1}
$$

to $K_{ \pm \nu}$. Thus, $\mathcal{A}_{ \pm \nu}\left(k+k_{\nu}^{ \pm}\left(\widehat{u}_{0}\right), u\right)$ may be considered a $2 \times 2$-matrix with respect to the basis $\left(\psi_{0}, \psi_{ \pm \nu}\right)$ (note that, technically, $\mathcal{A}_{ \pm \nu}\left(k+k_{\nu}^{ \pm}\left(\widehat{u}_{0}\right), u\right)$ is a bounded operator from a subspace of $\mathcal{F} \ell^{1}\left(\Gamma^{*}\right)$ to a subspace of $\left.\mathcal{F} \ell^{\infty, 1}\left(\Gamma^{*}\right)\right)$.

It is clear from the proof of proposition 4.5.15 that $\mathcal{A}_{ \pm \nu}\left(k+k_{\nu}^{ \pm}\left(\widehat{u}_{0}\right), u\right)$ measures the deviation from $S_{ \pm \nu}\left(k+k_{\nu}^{ \pm}\left(\widehat{u}_{0}\right), \widehat{u}_{0}\right)$ caused by the nonconstant potential. In particular, $\mathcal{A}_{ \pm \nu}\left(k+k_{\nu}^{ \pm}\left(\widehat{u}_{0}\right), u\right)$ is holomorphic in $k$, identically zero if $\bar{u}=0$.

Theorem 4.5.19. There is an open neighbourhood $V$ of $0 \in \mathbb{C}^{2}$ which depends only on $\Gamma^{*}$ such that for all $u_{0} \in \mathcal{F} \ell^{\infty, 1}\left(\Gamma^{*}\right)$ there is an $N \in \mathbb{N}$ and a $C>0$ with $u_{0} \in B_{C}^{N}$ as well as a $\delta>0$ such that for all $k \in V$, all $\nu \in \Gamma_{\delta}^{*}$ and all $u \in B_{C}^{N}$ the local part of the Fermi curve $F(u) \cap\left(V+k_{\nu}^{ \pm}\left(\widehat{u}_{0}\right)\right)$ is described by the zero locus of

$$
\begin{aligned}
& k \mapsto \operatorname{det}\left(\left(\begin{array}{cc}
-4 \pi^{2}\left(\left(k+k_{\nu}^{ \pm}\left(\widehat{u}_{0}\right)\right)^{2}+\widehat{u}_{0}\right) & 0 \\
0 & -4 \pi^{2}\left(\left(k+k_{\nu}^{\mp}\left(\widehat{u}_{0}\right)\right)^{2}+\widehat{u}_{0}\right)
\end{array}\right)\right. \\
& \left.+\mathcal{A}_{ \pm \nu}\left(k+k_{\nu}^{ \pm}\left(\widehat{u}_{0}\right), u\right)\right) .
\end{aligned}
$$

The same holds for all $\nu \in \Gamma^{*}, \nu \neq 0$, provided that $u$ is taken from a sufficiently small weakly open neighbourhood of the zero potential. 
Proof. Considering 4.5.7), we see that

$$
\begin{aligned}
S_{ \pm \nu}(k+ & \left.k_{\nu}^{ \pm}\left(\widehat{u}_{0}\right), \widehat{u}_{0}\right) \\
& =\left(\begin{array}{cc}
-4 \pi^{2}\left(\left(k+k_{\nu}^{ \pm}\left(\widehat{u}_{0}\right)\right)^{2}+\widehat{u}_{0}\right) & 0 \\
0 & -4 \pi^{2}\left(\left(k+k_{\nu}^{\mp}\left(\widehat{u}_{0}\right)\right)^{2}+\widehat{u}_{0}\right)
\end{array}\right)^{-1}
\end{aligned}
$$

because $\pm \nu=k_{\nu}^{\mp}\left(\widehat{u}_{0}\right)-k_{\nu}^{ \pm}\left(\widehat{u}_{0}\right)$. By theorem 3.3.24 the singular part of the resolvent remains contained in $K_{ \pm \nu}$ for potentials from a sufficiently small weakly open neighbourhood of a constant potential. For generic potentials this remains true for $\delta>0$ sufficiently small. Now, consider the case $k \notin F\left(4 \pi^{2} \widehat{u}_{0}\right)$. Then, by proposition 4.5 .15 for all $\nu \in \Gamma_{\delta}^{*}$, the part of the Fermi curve in $V+k_{\nu}^{ \pm}\left(\widehat{u}_{0}\right)$ is determined by the equation

$$
\operatorname{det}\left(1_{K_{ \pm \nu}}+S_{ \pm \nu}\left(k+k_{\nu}^{ \pm}\left(\widehat{u}_{0}\right), \widehat{u}_{0}\right) \mathcal{A}_{ \pm \nu}\left(k+k_{\nu}^{ \pm}\left(\widehat{u}_{0}\right), u\right)\right)=0 .
$$

Multiplication with

$$
\operatorname{det}\left(\left.\pi_{K_{ \pm \nu}}\left(\Delta_{k+k_{\nu}^{ \pm}\left(\widehat{u}_{0}\right)}-4 \pi^{2} \widehat{u}_{0}\right)\right|_{K_{ \pm \nu}}\right)
$$

now implies the theorem if $k+k_{\nu}^{ \pm}\left(\widehat{u}_{0}\right) \notin F\left(4 \pi^{2} \widehat{u}_{0}\right)$ because this determinant is nonzero in this case. For the $k \in F\left(4 \pi^{2} \widehat{u}_{0}\right)$ case, consider the left hand side of 4.5.17) as a meromorphic operator in $k$. The part

$$
\left(1-\left(1-\pi_{K_{ \pm \nu}}\right)\left(\Delta_{k+k_{\nu}^{ \pm}\left(\widehat{u}_{0}\right)}-4 \pi^{2} \widehat{u}_{0}\right)^{-1} \bar{u}\right)^{-1}
$$

is pole-free. For the remainder, we have

$$
\begin{aligned}
& \left(1-\pi_{K_{ \pm \nu}}\left(\Delta_{k+k_{\nu}^{ \pm}\left(\widehat{u}_{0}\right)}-4 \pi^{2} \widehat{u}_{0}\right)^{-1} \bar{u}\right. \\
& \left.\left(1-\left(1-\pi_{K_{ \pm \nu}}\right)\left(\Delta_{k+k_{\nu}^{ \pm}\left(\widehat{u}_{0}\right)}-4 \pi^{2} \widehat{u}_{0}\right)^{-1} \bar{u}\right)^{-1}\right)^{-1}\left(\Delta_{k+k_{\nu}^{ \pm}\left(\widehat{u}_{0}\right)}-4 \pi^{2} \widehat{u}_{0}\right)^{-1} \\
= & \left(\Delta_{k+k_{\nu}^{ \pm}\left(\widehat{u}_{0}\right)}-4 \pi^{2} \widehat{u}_{0}-\left(\Delta_{k+k_{\nu}^{ \pm}\left(\widehat{u}_{0}\right)}-4 \pi^{2} \widehat{u}_{0}\right) \pi_{K_{ \pm \nu}}\left(\Delta_{k+k_{\nu}^{ \pm}\left(\widehat{u}_{0}\right)}-4 \pi^{2} \widehat{u}_{0}\right)^{-1} \bar{u}\right. \\
& \left.\left(1-\left(1-\pi_{K_{ \pm \nu}}\right)\left(\Delta_{k+k_{\nu}^{ \pm}\left(\widehat{u}_{0}\right)}-4 \pi^{2} \widehat{u}_{0}\right)^{-1} \bar{u}\right)^{-1}\right)^{-1} \\
= & \left(\Delta_{k+k_{\nu}^{ \pm}\left(\widehat{u}_{0}\right)}-4 \pi^{2} \widehat{u}_{0}-\pi_{K_{ \pm \nu}} \bar{u}\left(1-\left(1-\pi_{K_{ \pm \nu}}\right)\left(\Delta_{k+k_{\nu}^{ \pm}\left(\widehat{u}_{0}\right)}-4 \pi^{2} \widehat{u}_{0}\right)^{-1} \bar{u}\right)^{-1}\right)^{-1} .
\end{aligned}
$$

The theorem now follows because the kernel of $\Delta_{k+k_{\nu}^{ \pm}\left(\widehat{u}_{0}\right)}-4 \pi^{2} \widehat{u}_{0}$ is contained in $K_{ \pm \nu}$ for $\nu \in \Gamma_{\delta}^{*}$.

This theorem greatly simplifies the local description of the Fermi curve we attained in theorem 4.1.3 in the asymptotic near-double-point case by remaining within the subspaces $K_{ \pm \nu}$. This enables us to draw our first simple conclusions about the geometric situation near the double points. Given some potential $u \in$ $\mathcal{F} \ell^{\infty, 1}\left(\Gamma^{*}\right)$, the matrix

$$
\begin{aligned}
& \left(\begin{array}{cc}
-4 \pi^{2}\left(\left(k+k_{\nu}^{ \pm}\left(\widehat{u}_{0}\right)\right)^{2}+\widehat{u}_{0}\right) & 0 \\
0 & -4 \pi^{2}\left(\left(k+k_{\nu}^{\mp}\left(\widehat{u}_{0}\right)\right)^{2}+\widehat{u}_{0}\right)
\end{array}\right) \\
& +\mathcal{A}_{ \pm \nu}\left(k+k_{\nu}^{ \pm}\left(\widehat{u}_{0}\right), u\right)
\end{aligned}
$$

must have, in dependence of $k+k_{\nu}^{ \pm}\left(\widehat{u}_{0}\right)$, at least one eigenvector with zero eigenvalue for the determinant to be zero and thus $k+k_{\nu}^{ \pm}\left(\widehat{u}_{0}\right) \in F(u)$. The straightforward generalisation of the definition of an unsplit double point at $k_{\nu}^{ \pm}\left(\widehat{u}_{0}\right)$ 
would be to demand the existence of two linearly independent eigenvectors with zero eigenvalue. In other words, the matrix would be the zero matrix. However, a further situation may occur, for proposition 3.1.7 does not generalise to arbitrary Schrödinger operators. If the matrix 4.5 .20 is not normal, it may happen that zero is its only eigenvalue, yet the matrix itself is nonzero. In this subsection and subsection 4.5.2, we shall keep this algebraic distinction in mind, and postpone the question of which definition of unsplit double point is geometrically the most sensible to subsection 4.5.3.

In order to further our understanding, let us investigate some properties of $\mathcal{A}_{ \pm \nu}$.

Lemma 4.5.21. There is an open neighbourhood $V$ of $0 \in \mathbb{C}^{2}$ which depends only on $\Gamma^{*}$ such that for all $u_{0} \in \mathcal{F} \ell^{\infty, 1}\left(\Gamma^{*}\right)$ there is an $N \in \mathbb{N}$ and a $C>0$ with $u_{0} \in B_{C}^{N}$ as well as a $\delta>0$ such that for all $k \in V$, all $\nu \in \Gamma_{\delta}^{*}$ and all $u \in B_{C}^{N}$, the matrix $\mathcal{A}_{ \pm \nu}\left(k+k_{\nu}^{ \pm}\left(\widehat{u}_{0}\right), u\right)$ is continuously differentiable in $k$, and we have

$$
\lim _{\|\nu\| \rightarrow \infty}\left\|\frac{\partial}{\partial k} \mathcal{A}_{ \pm \nu}\left(k+k_{\nu}^{ \pm}\left(\widehat{u}_{0}\right), u\right)\right\|=0
$$

uniformly.

Proof. By proposition 4.5.15. $\mathcal{A}_{ \pm \nu}\left(k+k_{\nu}^{ \pm}\left(\widehat{u}_{0}\right), u\right)$ exists and is holomorphic in $k$ for suitable $V, N, C$ and $\delta$. Now, set

$$
\begin{aligned}
A:=\bar{u}, \quad B & :=\left(1-\pi_{K_{ \pm \nu}}\right)\left(\Delta_{k+k_{\nu}^{ \pm}\left(\widehat{u}_{0}\right)}-4 \pi^{2} \widehat{u}_{0}\right)^{-1} \\
& =\left(\Delta_{k+k_{\nu}^{ \pm}\left(\widehat{u}_{0}\right)}-4 \pi^{2} \widehat{u}_{0}\right)^{-1}\left(1-\pi_{K_{ \pm \nu}}\right) .
\end{aligned}
$$

Denote the derivative with respect to $k$ with a prime. Then $A^{\prime}=0$ and $B^{\prime}=$ $-B \mathcal{C} B$, where $\mathcal{C}$ is the derivative of the restriction of the operator $\Delta_{k+k_{\nu}^{ \pm}\left(\widehat{u}_{0}\right)}-$ $4 \pi^{2} \widehat{u}_{0}$ to $K_{ \pm \nu}^{\perp}$. The Fourier transform of the components of the derivative of this operator is given by $-8 \pi^{2}\left(k_{i}+k_{\nu, i}^{ \pm}\left(\widehat{u}_{0}\right)+\kappa_{i}\right), i \in\{1,2\}$. In particular, the Fourier transform of $B \mathcal{C}$ is uniformly bounded. Now,

$$
\begin{aligned}
\left(A(1-B A)^{-1}\right)^{\prime} & =A\left((1-B A)^{-1}\right)^{\prime} \\
& =-A(1-B A)^{-1}(1-B A)^{\prime}(1-B A)^{-1} \\
& =A(1-B A)^{-1} B^{\prime} A(1-B A)^{-1} \\
& =-A(1-B A)^{-1} B C B A(1-B A)^{-1},
\end{aligned}
$$

so

$$
\begin{aligned}
& \frac{\partial}{\partial k} \mathcal{A}_{ \pm \nu}\left(k+k_{\nu}^{ \pm}\left(\widehat{u}_{0}\right), u\right) \\
&=-\pi_{K_{ \pm \nu}} \bar{u}\left(1-\left(\Delta_{k+k_{\nu}^{ \pm}\left(\widehat{u}_{0}\right)}-4 \pi^{2} \widehat{u}_{0}\right)^{-1}\left(1-\pi_{K_{ \pm \nu}}\right) \bar{u}\right)^{-1} \\
& B \mathcal{C}\left(1-\pi_{K_{ \pm \nu}}\right)\left(\Delta_{k+k_{\nu}^{ \pm}\left(\widehat{u}_{0}\right)}-4 \pi^{2} \widehat{u}_{0}\right)^{-1} \\
&\left.\bar{u}\left(1-\left(\Delta_{k+k_{\nu}^{ \pm}\left(\widehat{u}_{0}\right)}-4 \pi^{2} \widehat{u}_{0}\right)^{-1}\left(1-\pi_{K_{ \pm \nu}}\right) \bar{u}\right)^{-1}\right|_{K_{ \pm \nu}}
\end{aligned}
$$

By lemma 4.5 .13 and lemma 4.5.14, the norm of

$$
\left(1-\pi_{K_{ \pm \nu}}\right)\left(\Delta_{k+k_{\nu}^{ \pm}\left(\widehat{u}_{0}\right)}-4 \pi^{2} \widehat{u}_{0}\right)^{-1} \bar{u}
$$

vanishes uniformly as $\|\nu\| \rightarrow \infty$. The norm of $B \mathcal{C}$ and the remaining operators is uniformly bounded. This proves the statement. 
Before we go on, let us prove two generic operator identities similar to lemma 4.5.14

Lemma 4.5.23. Let $E_{1}, E_{2}$ be Banach spaces and let $A: E_{1} \rightarrow E_{2}, B: E_{2} \rightarrow E_{1}$ be continuous linear operators. Then $1-A B$ is boundedly invertible on $E_{2}$ if and only if $1-B A$ is boundedly invertible on $E_{1}$. If so, we have the two operator identities

$$
\begin{aligned}
(1-A B)^{-1} & =\left(1+A(1-B A)^{-1} B\right), \\
B(1-A B)^{-1} & =(1-B A)^{-1} B .
\end{aligned}
$$

Proof. Assume $1-B A$ to be boundedly invertible. Then

$$
\begin{aligned}
\left(1+A(1-B A)^{-1} B\right)(1-A B)= & 1+A(1-B A)^{-1} B \\
& -A B-A(1-B A)^{-1} B A B \\
= & 1+A\left((1-B A)^{-1}-1-(1-B A)^{-1} B A\right) B \\
= & 1+A\left((1-B A)^{-1}(1-B A)-1\right) B \\
= & 1+A(1-1) B=1, \\
(1-A B)\left(1+A(1-B A)^{-1} B\right)= & 1-A B+A(1-B A)^{-1} B \\
& -A B A(1-B A)^{-1} B \\
= & 1-A\left(1-(1-B A)^{-1}+B A(1-B A)^{-1}\right) B \\
= & 1-A\left(1-(1-B A)(1-B A)^{-1}\right) B \\
= & 1-A(1-1) B=1 .
\end{aligned}
$$

Therefore, $1-A B$ is boundedly invertible. The reverse statement follows in the same way. This calculation also shows the first of the stipulated operator identities. For we second, we have

$$
\begin{aligned}
B(1-A B)^{-1} & =B\left(1+A(1-B A)^{-1} B\right) \\
& =B+B A(1-B A)^{-1} B \\
& =\left(1+B A(1-B A)^{-1}\right) B \\
& =\left(1+(1-B A)^{-1}-(1-B A)^{-1}+B A(1-B A)^{-1}\right) B \\
& =\left(1+(1-B A)^{-1}-(1-B A)(1-B A)^{-1}\right) B \\
& =\left(1+(1-B A)^{-1}-1\right) B=(1-B A)^{-1} B .
\end{aligned}
$$

Proposition 4.5.24. Let $u \in \mathcal{F} \ell^{\infty, 1}\left(\Gamma^{*}\right), \nu \in \Gamma^{*}$ and let $V \subseteq \mathbb{C}^{2}$ with $V=-V$ such that $\mathcal{A}_{ \pm \nu}\left(k+k_{\nu}^{ \pm}\left(\widehat{u}_{0}\right), u\right)$ is properly defined for all $k \in V$ in the sense of definition 4.5.18. Then

$$
\begin{aligned}
\mathcal{A}_{ \pm \nu}\left(k+k_{\nu}^{ \pm}\left(\widehat{u}_{0}\right), u\right)^{t} & =\mathcal{A}_{\mp \nu}\left(-k+k_{-\nu}^{ \pm}\left(\widehat{u}_{0}\right), u\right), \\
\left(\begin{array}{cc}
0 & 1 \\
1 & 0
\end{array}\right) \mathcal{A}_{ \pm \nu}\left(k+k_{\nu}^{ \pm}\left(\widehat{u}_{0}\right), u\right)\left(\begin{array}{cc}
0 & 1 \\
1 & 0
\end{array}\right) & =\mathcal{A}_{\mp \nu}\left(k+k_{\nu}^{\mp}\left(\widehat{u}_{0}\right), u\right) .
\end{aligned}
$$


Proof. The first equation follows from theorem 4.5.19 and proposition 4.5.8. It can also be seen directly using lemma 4.5 .23 for we have $\pi_{K_{ \pm \nu}}^{t}=\pi_{K_{\mp \nu}}$ and

$$
\begin{aligned}
\left(\bar{u}\left(1-\left(\Delta_{k+k_{\nu}^{ \pm}\left(\widehat{u}_{0}\right)}-4 \pi^{2} \widehat{u}_{0}\right)^{-1}\left(1-\pi_{K_{ \pm \nu}}\right) \bar{u}\right)^{-1}\right)^{t} & \\
& =\left(1-\bar{u}\left(\Delta_{-k-k_{\nu}^{ \pm}\left(\widehat{u}_{0}\right)}-4 \pi^{2} \widehat{u}_{0}\right)^{-1}\left(1-\pi_{K_{\mp \nu}}\right)\right)^{-1} \bar{u}
\end{aligned}
$$

For the second equation, consider the lattice shift by $\pm \nu$. We have $\psi_{ \pm \nu} \bar{u} \psi_{\mp \nu}=\bar{u}$ and

$$
\begin{aligned}
\psi_{ \pm \nu} S_{ \pm \nu}\left(k+k_{\nu}^{ \pm}\left(\widehat{u}_{0}\right), \widehat{u}_{0}\right) \psi_{\mp \nu} & =S_{\mp \nu}\left(k+k_{\nu}^{\mp}\left(\widehat{u}_{0}\right), \widehat{u}_{0}\right) \\
& =\left(\begin{array}{cc}
0 & 1 \\
1 & 0
\end{array}\right) S_{ \pm \nu}\left(k+k_{\nu}^{ \pm}\left(\widehat{u}_{0}\right), \widehat{u}_{0}\right)\left(\begin{array}{cc}
0 & 1 \\
1 & 0
\end{array}\right)
\end{aligned}
$$

by equation 4.5.7.

Note that in this proposition, the matrices

$$
\left(\begin{array}{ll}
0 & 1 \\
1 & 0
\end{array}\right)
$$

map $K_{ \pm \nu}$ into $K_{\mp \nu}$ and might therefore be more properly denoted by

$$
\left(\begin{array}{ll}
0 & 1 \\
1 & 0
\end{array}\right)_{ \pm} .
$$

We shall omit this additional disambiguation, however.

Theorem 4.5.25. There is an open neighbourhood $V$ of $0 \in \mathbb{C}^{2}$ which depends only on $\Gamma^{*}$ such that for all $k \in V$ and all $\nu \in \Gamma^{*}, \nu \neq 0$, the off-diagonal entries of the functional variation of $\mathcal{A}_{ \pm \nu}$ around the zero potential (or even a constant potential for $\nu$ with sufficiently large norm) can be regarded as a nonlinear perturbation of the Fourier transform. More precisely, the variation of $\mathcal{A}_{ \pm \nu}$ at $u=0$ has the form

$$
f \mapsto\left(\begin{array}{cc}
0 & \widehat{f}( \pm \nu) \\
\widehat{f}(\mp \nu) & 0
\end{array}\right) .
$$

In general, for all $u_{0} \in \mathcal{F} \ell^{\infty, 1}\left(\Gamma^{*}\right)$ there is an $N \in \mathbb{N}$ and $a C>0$ with $u_{0} \in B_{C}^{N}$ as well as a $\delta>0$ such that the variation of $\mathcal{A}_{ \pm \nu}$ is defined for all $\nu \in \Gamma_{\delta}^{*}$ at all $u \in B_{C}^{N}$ and has the form

$$
\begin{aligned}
f \mapsto & \pi_{K_{ \pm \nu}}\left(1-\bar{u}\left(1-\pi_{K_{ \pm \nu}}\right)\left(\Delta_{k+k_{\nu}^{ \pm}\left(\widehat{u}_{0}\right)}-4 \pi^{2} \widehat{u}_{0}\right)^{-1}\right)^{-1} \\
& \cdot\left(\left.\left(1-\pi_{K_{0}}\right) f\right|_{K_{0}^{\perp}}-\bar{u}\left(1-\pi_{K_{ \pm \nu}}\right)\left(\Delta_{k+k_{\nu}^{ \pm}\left(\widehat{u}_{0}\right)}-4 \pi^{2} \widehat{u}_{0}\right)^{-1} \mathcal{C}(f)\right. \\
& \left.\cdot\left(\Delta_{k+k_{\nu}^{ \pm}\left(\widehat{u}_{0}\right)}-4 \pi^{2} \widehat{u}_{0}\right)^{-1}\left(1-\pi_{K_{ \pm \nu}}\right) \bar{u}\right) \\
& \left.\cdot\left(1-\left(\Delta_{k+k_{\nu}^{ \pm}\left(\widehat{u}_{0}\right)}-4 \pi^{2} \widehat{u}_{0}\right)^{-1}\left(1-\pi_{K_{ \pm \nu}}\right) \bar{u}\right)^{-1}\right|_{K_{ \pm \nu}}
\end{aligned}
$$

where $\mathcal{C}$ is a (with respect to $\nu$ ) uniformly bounded linear operator mapping (left multiplications with) $\mathcal{F} \ell^{\infty, 1}\left(\Gamma^{*}\right)$-functions into (left multiplications with) $\mathcal{F} \ell^{\infty, 1}\left(\Gamma^{*}\right)$-functions. 
Proof. As in the proof of lemma 4.5.21, we can define the operators $A$ and $B$ as in 4.5.22). This time, we use a prime to denote the variation with respect to $u$. Then $A^{\prime}$ is given by the operation $\left.f \mapsto\left(1-\pi_{K_{0}}\right) f\right|_{K_{0}^{\perp}}$. The derivative of $B$ again has the form $B^{\prime}=-B \mathcal{C} B$, where this time, a simple calculation shows that the Fourier transform of $\mathcal{C}$ at $\kappa \neq 0, \pm \nu$ is given by

$$
\left.\widehat{f} \mapsto \frac{-8 \pi^{2} i}{\|\nu\|^{2} \xi\left(\widehat{u}_{0}, \nu\right)}\left(\kappa+k+k_{\nu}^{ \pm}\left(\widehat{u}_{0}\right) \mid\left(\nu_{2},-\nu_{1}\right)\right) \widehat{\pi}_{K_{0}} \widehat{f}\right|_{K_{0}}-\left.4 \pi^{2} \widehat{\pi}_{K_{0}} \widehat{f}\right|_{K_{0}} .
$$

Clearly, this expression is bounded with respect to $\nu$, and shows that $\mathcal{C}$ maps $\mathcal{F} \ell^{\infty, 1}\left(\Gamma^{*}\right)$-functions to $\mathcal{F} \ell^{\infty, 1}\left(\Gamma^{*}\right)$-functions. Now, by lemma 4.5.23, we have

$$
\begin{aligned}
\left(A(1-B A)^{-1}\right)^{\prime} & =A^{\prime}(1-B A)^{-1}+A\left((1-B A)^{-1}\right)^{\prime} \\
& =A^{\prime}(1-B A)^{-1}-A(1-B A)^{-1}(1-B A)^{\prime}(1-B A)^{-1} \\
& =A^{\prime}(1-B A)^{-1}+A(1-B A)^{-1}\left(B^{\prime} A+B A^{\prime}\right)(1-B A)^{-1} \\
& =\left(A^{\prime}+A(1-B A)^{-1} B^{\prime} A+A(1-B A)^{-1} B A^{\prime}\right)(1-B A)^{-1} \\
& =\left(\left(1+A(1-B A)^{-1} B\right) A^{\prime}+(1-A B)^{-1} A B^{\prime} A\right)(1-B A)^{-1} \\
& =\left((1-A B)^{-1} A^{\prime}+(1-A B)^{-1} A B^{\prime} A\right)(1-B A)^{-1} \\
& =(1-A B)^{-1}\left(A^{\prime}-A B C B A\right)(1-B A)^{-1} .
\end{aligned}
$$

This shows the general case 4.5.27. At $u=0, \mathcal{A}_{ \pm \nu}$ is defined for all $\nu \in \Gamma^{*}$, giving the variation

$$
\left.\left.f \mapsto \pi_{K_{ \pm \nu}}\left(1-\pi_{K_{0}}\right) f\right|_{K_{0}^{\perp}}\right|_{K_{ \pm \nu}},
$$

which is precisely 4.5 .26 with respect to the bases $\left(\psi_{0}, \psi_{ \pm \nu}\right)$.

\subsubsection{Approximation with potentials of finite type}

In theorem 4.5 .19 we found a description of the Fermi curves $F(u)$ near the double points $k_{\nu}^{ \pm}\left(\widehat{u}_{0}\right)$, for sufficiently large $\|\nu\|$. In theorem 4.5.25, we found a variation which essentially generates the Fourier transform operator at the zero potential. We shall render the description from the former theorem more explicit by attaching "perturbed" Fourier transforms to variations of $\mathcal{A}_{ \pm \nu}$ at nonzero potentials, again for sufficiently large $\|\nu\|$.

Proposition 4.5.29. There is an open neighbourhood $V$ of $0 \in \mathbb{C}^{2}$ which depends only on $\Gamma^{*}$ such that for all $u_{0} \in \mathcal{F} \ell^{\infty, 1}\left(\Gamma^{*}\right)$ there is an $N \in \mathbb{N}$ and a $C>0$ with $u_{0} \in B_{C}^{N}$ as well as a $\delta>0$ such that for all $u \in B_{C}^{N}$ and all $\nu \in \Gamma_{\delta}^{*}$ there is a unique $k_{ \pm \nu} \in V$ such that the diagonal entries of the matrix (4.5.20) become zero at $k=k_{ \pm \nu}$.

Proof. Consider the system of equations

$$
\left(k^{\prime}+k_{\nu}^{+}\left(\widehat{u}_{0}\right)\right)^{2}+\widehat{u}_{0}=d_{1}(k), \quad\left(k^{\prime}+k_{\nu}^{-}\left(\widehat{u}_{0}\right)\right)^{2}+\widehat{u}_{0}=d_{2}(k),
$$

where $d_{1}(k)$ and $d_{2}(k)$ are the diagonal entries of $-\mathcal{A}_{\nu}\left(k+k_{\nu}^{+}\left(\widehat{u}_{0}\right), u\right) / 4 \pi^{2}$. Algebraic considerations show that for each valid $k$, there are exactly two solutions $k^{\prime}$ of this system bounded away from each other if the norm of $\nu$ is much larger 
than the norms of $d_{1}(k)-\widehat{u}_{0}$ and $d_{2}(k)-\widehat{u}_{0}$. If $u=0$, then $d_{1}(k)=d_{2}(k)=0$. In this case, we have the two trivial solutions $k^{\prime}=0$ and $k^{\prime}=-k_{\nu}^{+}-k_{\nu}^{-}$. For $u \neq 0$ we get a perturbation of the trivial solutions. By theorem 4.5.25 this perturbation is arbitrarily small for sufficiently large $\nu \in \Gamma^{*}$ because $\ell^{\infty, 1}\left(\Gamma^{*}\right)$ sequences vanish near infinity. In particular, the two solutions are still bounded away from each other, with increasing distance as $\nu$ increases. Expanding the left hand sides of our system of equations, we get

$$
\begin{aligned}
\left(k_{1}^{\prime}\right)^{2}+\left(k_{2}^{\prime}\right)^{2}+k_{1}^{\prime}\left(-\nu_{1}+i \nu_{2} \xi\left(\widehat{u}_{0}, \nu\right)\right)+k_{2}^{\prime}\left(-i \nu_{1} \xi\left(\widehat{u}_{0}, \nu\right)-\nu_{2}\right) & =d_{1}(k), \\
\left(k_{1}^{\prime}\right)^{2}+\left(k_{2}^{\prime}\right)^{2}+k_{1}^{\prime}\left(\nu_{1}+i \nu_{2} \xi\left(\widehat{u}_{0}, \nu\right)\right)+k_{2}^{\prime}\left(-i \nu_{1} \xi\left(\widehat{u}_{0}, \nu\right)+\nu_{2}\right) & =d_{2}(k) .
\end{aligned}
$$

Define

$$
\begin{aligned}
F: \mathbb{C}^{2} \times \mathbb{C}^{2} & \rightarrow \mathbb{C}^{2} \\
\left(k, k^{\prime}\right) & \mapsto\left(\begin{array}{c}
\left(k_{1}^{\prime}\right)^{2}+\left(k_{2}^{\prime}\right)^{2}+k_{1}^{\prime}\left(-\nu_{1}+i \nu_{2} \xi\left(\widehat{u}_{0}, \nu\right)\right) \\
+k_{2}^{\prime}\left(-i \nu_{1} \xi\left(\widehat{u}_{0}, \nu\right)-\nu_{2}\right)-d_{1}(k) \\
\left(k_{1}^{\prime}\right)^{2}+\left(k_{2}^{\prime}\right)^{2}+k_{1}^{\prime}\left(\nu_{1}+i \nu_{2} \xi\left(\widehat{u}_{0}, \nu\right)\right) \\
+k_{2}^{\prime}\left(-i \nu_{1} \xi\left(\widehat{u}_{0}, \nu\right)+\nu_{2}\right)-d_{2}(k)
\end{array}\right) .
\end{aligned}
$$

By lemma 4.5.21, $F$ is continuously differentiable in $V \times \mathbb{C}^{2}$ for a suitable $V$. Now,

$$
\frac{\partial F}{\partial k^{\prime}}\left(k, k^{\prime}\right)=\left(\begin{array}{cc}
2 k_{1}^{\prime}-\nu_{1}+i \nu_{2} \xi\left(\widehat{u}_{0}, \nu\right) & 2 k_{2}^{\prime}-i \nu_{1} \xi\left(\widehat{u}_{0}, \nu\right)-\nu_{2} \\
2 k_{1}^{\prime}+\nu_{1}+i \nu_{2} \xi\left(\widehat{u}_{0}, \nu\right) & 2 k_{2}^{\prime}-i \nu_{1} \xi\left(\widehat{u}_{0}, \nu\right)+\nu_{2}
\end{array}\right) .
$$

The determinant of this operator is given by

$$
\begin{aligned}
4 k_{1}^{\prime} \nu_{2}-4 k_{2}^{\prime} \nu_{1}+2 i \nu_{1}^{2} \xi\left(\widehat{u}_{0}, \nu\right)+2 i \nu_{2}^{2} \xi\left(\widehat{u}_{0}, \nu\right) & \\
= & 4\left(k_{1}^{\prime} \nu_{2}-k_{2}^{\prime} \nu_{1}\right)+2 i \xi\left(\widehat{u}_{0}, \nu\right)\|\nu\|^{2} .
\end{aligned}
$$

Now, the imaginary part of $V$ is bounded and by the considerations above, for all $\nu \in \Gamma^{*}$ with sufficiently large norm, the solutions $k^{\prime}$ belonging to these $k$ also have bounded imaginary part. Furthermore, $\xi\left(\widehat{u}_{0}, \nu\right)$ approaches 1 for large $\nu$. Therefore, the determinant is nonzero for large $\nu$. Hence, by the implicit function theorem, we can patch together a unique function $f: V \rightarrow \mathbb{C}^{2}$ such that $F(k, f(k))=0$ for all $k \in V$. Furthermore,

$$
\frac{\partial f}{\partial k}(k)=-\left(\frac{\partial F}{\partial k^{\prime}}(k, f(k))\right)^{-1} \frac{\partial F}{\partial k}(k, f(k)) .
$$

Now, the derivative of $F$ with respect to $k$ is essentially given by the derivatives of $d_{1}$ and $d_{2}$ (up to sign) and so is small by lemma 4.5.21. By 4.5.30, the inverse of the derivative of $F$ with respect to $k^{\prime}$ is bounded. Hence, by the mean value theorem, $f$ is Lipschitz continuous with arbitrarily small Lipschitz constant as $\|\nu\| \rightarrow \infty$. Furthermore, $f(0) \rightarrow 0$ as $\|\nu\| \rightarrow \infty$. Therefore we may assume that $f(V) \subseteq V$, and that the same holds for a slightly smaller closed subset of $V$. Hence, $f$ has a unique fixed point which does what we want by Banach's fixed point theorem. Analogous statements hold for the other choices of signs. 
Definition 4.5.31. Given the previous proposition, we denote the off-diagonal entries of $\mathcal{A}_{ \pm \nu}\left(k_{ \pm \nu}+k_{\nu}^{ \pm}\left(\widehat{u}_{0}\right), u\right)$ by $\widehat{u}_{1}(u, \pm \nu)^{ \pm}$and $\widehat{u}_{2}(u, \pm \nu)^{ \pm}$. At first glance, this looks like four different functions for each eligible $u$. However, the uniqueness of $k_{ \pm \nu}$ together with proposition 4.5 .24 immediately shows that

$$
\widehat{u}_{1}(u, \nu)^{+}=\widehat{u}_{2}(u,-\nu)^{+}=\widehat{u}_{1}(u,-\nu)^{-}=\widehat{u}_{2}(u, \nu)^{-}
$$

(in particular, all these functions have equal decreasing rearrangement). Therefore, it is appropriate to set $\widehat{u}(u, \nu):=\widehat{u}_{1}(u, \nu)^{+}$and express the previous four functions in terms of $\widehat{u}(u, \nu)$. The off-diagonalised form of $\mathcal{A}_{ \pm \nu}$ near $k_{\nu}^{ \pm}\left(\widehat{u}_{0}\right)$ then becomes

$$
\left(\begin{array}{cc}
0 & \widehat{u}(u, \pm \nu) \\
\widehat{u}(u, \mp \nu) & 0
\end{array}\right)
$$

which is reminiscent of 4.5.26). In light of subsection 4.5.1. we call $\widehat{u}(u, \nu)$ the perturbed Fourier transform of $u$.

In particular, $\widehat{u}(0, \nu)=0$, and for $\nu$ with large norm, $\widehat{u}(u, \nu)$ approximates the regular Fourier transform $\widehat{u}(\nu)$.

Definition 4.5.32. We say that a potential $u \in \mathcal{F} \ell^{\infty, 1}\left(\Gamma^{*}\right)$ has finite type if the perturbed Fourier transform of $u$ fulfils

$$
\widehat{u}(u, \nu)=0 \quad \text { and } \quad \widehat{u}(u,-\nu)=0
$$

for all but finitely many of those $\nu \in \Gamma^{*}$ for which this condition makes sense.

In order to understand the subset of finite-type potentials better, we shall now determine the function space the perturbed Fourier transform resides in.

Lemma 4.5.33. There is an open neighbourhood $V$ of $0 \in \mathbb{C}$ which depends only on $\Gamma^{*}$ such that for all $u_{0} \in \mathcal{F} \ell^{\infty, 1}\left(\Gamma^{*}\right)$ there is an $N \in \mathbb{N}$ and $a C>0$ with $u_{0} \in$ $B_{C}^{N}$ as well as a $\delta>0$ such that for all $k \in V$, all $\nu \in \Gamma^{*}$ and all $u \in B_{C}^{N}$ the operator $\left(\Delta_{k+k_{\nu}^{ \pm}\left(\widehat{u}_{0}\right)}-4 \pi^{2} \widehat{u}_{0}\right)^{-1}\left(1-\pi_{K_{ \pm \nu}}\right) \bar{u}$ maps $\ell^{\infty}\left(\Gamma^{*}\right) \widehat{\otimes} \mathcal{F} \ell^{1}\left(\Gamma^{*}\right)$ boundedly into $c^{0}\left(\Gamma^{*}\right) \widehat{\otimes} \mathcal{F} \ell^{1}\left(\Gamma^{*}\right)$ (the left tensor factor belonging to the variable $\nu$ ). The restriction from $\Gamma^{*}$ to $\Gamma_{\delta}^{*}$ with respect to $\nu$ yields an operator norm smaller than one.

Proof. This is an extension of lemma 4.5.9. We have $c^{0}\left(\Gamma^{*}\right) \cdot \ell^{\infty}\left(\Gamma^{*}\right) \subseteq c^{0}\left(\Gamma^{*}\right)$. Continuity now follows from proposition 2.6.13 and corollaries 2.6.15 and 2.6.16.

This lemma is sufficient to prove that the entries of $\mathcal{A}_{ \pm \nu}\left(k+k_{\nu}^{ \pm}\left(\widehat{u}_{0}\right), u\right)$ are $c^{0}\left(\Gamma_{\delta}^{*}\right)$ with respect to $\nu$. However, as $u \in \mathcal{F} \ell^{\infty, 1}\left(\Gamma^{*}\right)$, we would rather like to have the entries in $\ell^{\infty, 1}\left(\Gamma^{*}\right)$. This will be the case either because $u$ is sufficiently regular, or because we can derive sufficient regularity from (4.5.11). In order to avoid a case analysis based on these two options, let us instead estimate $u$ against a potential with limited decay behaviour.

Definition 4.5.34. Let $u \in \mathcal{F} \ell^{\infty, 1}\left(\Gamma^{*}\right)$. Then we define the sequence $\left(\widetilde{u}_{n}\right)_{n \in \mathbb{N}}$ recursively via

$$
\widetilde{u}_{1}:=\widehat{u}^{\star}(1), \quad \widetilde{u}_{n+1}:=\max \left\{\widehat{u}^{\star}(n+1), \sqrt{\frac{n}{n+1}} \widetilde{u}_{n}\right\} .
$$


Proposition 4.5.35. Let $u \in \mathcal{F} \ell^{\infty, 1}\left(\Gamma^{*}\right)$ be not identically zero. Then the corresponding sequence $\left(\widetilde{u}_{n}\right)_{n \in \mathbb{N}}$ is decreasing and positive. Moreover, we have

$$
\left\|\left(\widetilde{u}_{n}\right)_{n \in \mathbb{N}}\right\|_{\infty, 1} \leq \frac{4}{1-\frac{1}{\sqrt{2}}}\|u\|_{\mathcal{F} \infty, 1},
$$

where the norm on the left hand side is the norm of the Luxemburg representation space $\ell^{\infty, 1}(\mathbb{N})$. In particular, there is a potential in $\mathcal{F} \ell^{\infty, 1}\left(\Gamma^{*}\right)$ whose Fourier transform has a decreasing rearrangement equal to $\left(\widetilde{u}_{n}\right)_{n \in \mathbb{N}}$.

Proof. The sequence is obviously positive. Let $n \in \mathbb{N}$. Consider the case

$$
\widetilde{u}_{n+1}=\sqrt{\frac{n}{n+1}} \widetilde{u}_{n}
$$

first. Then

$$
\widetilde{u}_{n}-\widetilde{u}_{n+1}=\left(1-\sqrt{\frac{n}{n+1}}\right) \widetilde{u}_{n} \geq 0 .
$$

The other case is $\widetilde{u}_{n+1}=\widehat{u}^{\star}(n+1)$. Then

$$
\widetilde{u}_{n}-\widetilde{u}_{n+1}=\widetilde{u}_{n}-\widehat{u}^{\star}(n+1) \geq \widehat{u}^{\star}(n)-\widehat{u}^{\star}(n+1) \geq 0 .
$$

Therefore, $\left(\widetilde{u}_{n}\right)_{n \in \mathbb{N}}$ is decreasing. Let us now estimate this sequence. Thereto, we introduce a helper sequence $\left(a_{n}\right)_{n \in \mathbb{N}}$ as follows:

$$
a_{n}:= \begin{cases}0 & \text { if } \widetilde{u}_{n}=\widehat{u}^{\star}(n), \\ 1 & \text { otherwise }\end{cases}
$$

Then the first element of $\left(a_{n}\right)_{n \in \mathbb{N}}$ is a zero, and the entire sequence consists of at most countably many stretches of zeros and ones. We enumerate these stretches separately in the canonical order. Now, by Cauchy's condensation theorem, we have

$$
\sum_{n=1}^{\infty} \frac{\widetilde{u}_{n}}{n} \leq \sum_{n=0}^{\infty} \widetilde{u}_{2^{n}} \leq 2 \sum_{n=1}^{\infty} \frac{\widetilde{u}_{n}}{n} .
$$

It is therefore sufficient to estimate the middle expression. In particular, we may alter $\left(\widetilde{u}_{n}\right)_{n \in \mathbb{N}}$ between subsequent powers of two as long as the decreasing property is retained. Thus, for all $m_{1}, m_{2} \in \mathbb{N}$ with no power of two in between (excluding $m_{1}$ and $m_{2}$ themselves), such that $a_{m_{1}}=a_{m_{2}}=0$, we may assume $a_{m}=0$ for all $m$ between $m_{1}$ and $m_{2}$. Hence, the positional range of every stretch of ones includes a power of two. In particular, the $n$-th stretch of ones starts beyond the $2^{n-1}$-st element of the sequence $\left(a_{n}\right)_{n \in \mathbb{N}}$. Now, let $b_{n}$ be the sum of all $\widetilde{u}_{2^{m}}$ such that $a_{2^{m}}$ belongs to the $n$-th stretch of zeros (if the sum is empty or if there is no $n$-th stretch of zeros, we set $\left.b_{n}=0\right)$. Likewise, let $c_{n}$ be the sum of all $\widetilde{u}_{2^{m}}$ such that $a_{2^{m}}$ belongs to the $n$-th stretch of ones (or $c_{n}=0$ if not applicable). That is, we have

$$
b_{n}=\widehat{u}^{\star}\left(2^{m_{0}}\right)+\widehat{u}^{\star}\left(2^{m_{0}+1}\right)+\cdots+\widehat{u}^{\star}\left(2^{m_{1}}\right)
$$

if the $n$-th stretch of zeros starts anywhere from the $\left(2^{m_{0}-1}+1\right)$-st to the $2^{m_{0}}$-th position and ends anywhere from the $2^{m_{1}}$-th to the $\left(2^{m_{1}+1}-1\right)$-st position (if 
the stretch does not end, the sum is the limit of an infinite series). Due to the way we recursively defined $\widetilde{u}_{n}$, we have likewise

$$
c_{n}=\widetilde{u}_{2^{m_{0}}}\left(1+2^{-1 / 2}+\cdots+2^{\left(m_{1}-m_{0}\right) / 2}\right),
$$

again with the sum becoming the limit of an infinite series if the stretch does not end. We then have

$$
\sum_{n=1}^{\infty} b_{n} \leq \sum_{n=0}^{\infty} \widehat{u}^{\star}\left(2^{n}\right) .
$$

Due to our considerations above, we have $\widetilde{u}_{2^{m_{0}}} \leq \widehat{u}^{\star}\left(2^{n-1}\right)$ in 4.5 .36 and thus

$$
\sum_{n=1}^{\infty} c_{n} \leq \frac{1}{1-\frac{1}{\sqrt{2}}} \sum_{n=0}^{\infty} \widehat{u}^{\star}\left(2^{n}\right)
$$

Now,

$$
\sum_{n=0}^{\infty} \widetilde{u}_{2^{n}} \leq \sum_{n=1}^{\infty}\left(b_{n}+c_{n}\right) \leq \frac{4}{1-\frac{1}{\sqrt{2}}}\|u\|_{\mathcal{F} \infty, 1},
$$

proving the proposition.

Definition 4.5.37. We denote the function 4.5.11 by $g(k, \nu, \rho)$. Let $g^{\star}(k, \nu, n)$ be the decreasing rearrangement of $g$ with respect to $\rho$. Since $\Gamma^{*}$ is twodimensional, we have the estimate

$$
g^{\star}(k, \nu, n) \leq C \frac{1}{n+\sqrt{n}\|\nu\|+\|\nu\|^{2}} \leq C \frac{1}{n+\|\nu\|^{2}},
$$

where $C$ does not depend on $n, \nu, k$ or $\widehat{u}_{0}$ (recall that $k$ comes from a bounded domain, and by monotonicity likewise $\widehat{u}_{0}$ through $u$ ). Likewise, there is also an enumeration $\left(\nu_{m}\right)_{m \in \mathbb{N}}$ of the elements of $\Gamma^{*}$ such that

$$
g^{\star}\left(k, \nu_{m}, n\right) \leq C^{\prime} \frac{1}{n+m}
$$

with an independent constant $C^{\prime}>0$. Let $u \in \mathcal{F} \ell^{\infty, 1}\left(\Gamma^{*}\right)$. By proposition 4.5.35, $\widetilde{u}_{n}$ converges to zero. Therefore, for each $m \in \mathbb{N}$ there exists a not necessarily unique $n \in \mathbb{N}$ such that the supremum

$$
\sup _{n \in \mathbb{N}} \widetilde{u}_{n} \frac{n}{n+m}
$$

is attained. Picking one such $n$ arbitrarily defines a sequence $n(m)$ for each $u$.

Lemma 4.5.39. Let $u \in \mathcal{F} \ell^{\infty, 1}\left(\Gamma^{*}\right)$ be not identically zero. Then each corresponding sequence $n(m)$ is increasing. This remains true if we replace $\widetilde{u}_{n}$ with any positive sequence converging to zero.

Proof. Let $m \in \mathbb{N}$ and set $n_{0}:=n(m)$. Since the supremum in 4.5.38 is attained at $n=n_{0}$, we have

$$
\widetilde{u}_{n_{0}} \frac{n_{0}}{n_{0}+m}-\widetilde{u}_{n_{0}-a} \frac{n_{0}-a}{n_{0}-a+m} \geq 0
$$


for all $a \in \mathbb{N}$ with $0<a<n_{0}$. This inequality implies

$$
\frac{\widetilde{u}_{n_{0}}}{\widetilde{u}_{n_{0}-a}} \geq \frac{\left(n_{0}-a\right)\left(n_{0}+m\right)}{n_{0}\left(n_{0}-a+m\right)} .
$$

Now, $a>0$ implies

$$
\begin{aligned}
\left(n_{0}-a+m\right)\left(n_{0}+m\right)+\left(n_{0}+m\right) & >\left(n_{0}-a+m\right)\left(n_{0}+m\right)+\left(n_{0}-a+m\right) \\
\Rightarrow \quad\left(n_{0}-a+m+1\right)\left(n_{0}+m\right) & >\left(n_{0}-a+m\right)\left(n_{0}+m+1\right) \\
\Rightarrow \quad \frac{n_{0}+m}{n_{0}-a+m} & >\frac{n_{0}+m+1}{n_{0}-a+m+1}
\end{aligned}
$$

and thus

$$
\begin{aligned}
\frac{\widetilde{u}_{n_{0}}}{\widetilde{u}_{n_{0}-a}} & >\frac{\left(n_{0}-a\right)\left(n_{0}+m+1\right)}{n_{0}\left(n_{0}-a+m+1\right)} \\
\Rightarrow \quad \widetilde{u}_{n_{0}} \frac{n_{0}}{n_{0}+m+1} & >\widetilde{u}_{n_{0}-a} \frac{n_{0}-a}{n_{0}-a+m+1} .
\end{aligned}
$$

Hence $n(m+1) \geq n_{0}=n(m)$, so $n(m)$ is increasing.

Lemma 4.5.40. Let $u \in \mathcal{F} \ell^{\infty, 1}\left(\Gamma^{*}\right)$ be not identically zero. Then for each corresponding sequence $n(m)$ we have $n(m) \geq m$.

Proof. We prove this by induction. Certainly, $n(1) \geq 1$. Let $m \in \mathbb{N}$ and assume $n(m) \geq m$. We must prove that $n(m+1) \geq m+1$. If $n(m)>m$, then $n(m+1) \geq m+1$ by lemma 4.5.39. It remains to investigate the case $n_{0}:=$ $n(m)=m$. We have

$$
\begin{aligned}
m+1 & >0 \\
\Rightarrow \quad m^{2}+2 m+1 & >n_{0}^{2}+n_{0} \\
\Rightarrow \quad n_{0}^{3}+2 n_{0}^{2} m+3 n_{0}^{2}+n_{0} m^{2} & >n_{0}^{3}+2 n_{0}^{2} m+4 n_{0}^{2}+n_{0} m^{2}+4 n_{0} m+4 n_{0} \\
\left.+4 n_{0} m+3 n_{0}+m^{2}+2 m+1\right)^{2} & >n_{0}\left(n_{0}+m+2\right)^{2} \\
\Rightarrow \quad\left(n_{0}+1\right)\left(n_{0}+m+1\right)^{2} & >\frac{n_{0}\left(n_{0}+1+m+1\right)^{2}}{\left(n_{0}+1\right)\left(n_{0}+m+1\right)^{2}} \\
\Rightarrow \quad \frac{n_{0}}{n_{0}+1} & >\frac{n_{0}^{2}\left(n_{0}+1+m+1\right)^{2}}{\left(n_{0}+1\right)^{2}\left(n_{0}+m+1\right)^{2}} \\
\Rightarrow \quad \sqrt{\frac{n_{0}}{n_{0}+1}} & >\frac{n_{0}\left(n_{0}+1+m+1\right)}{\left(n_{0}+1\right)\left(n_{0}+m+1\right)} .
\end{aligned}
$$

But by construction,

$$
\frac{\widetilde{u}_{n_{0}+1}}{\widetilde{u}_{n_{0}}} \geq \sqrt{\frac{n_{0}}{n_{0}+1}}>\frac{n_{0}\left(n_{0}+1+m+1\right)}{\left(n_{0}+1\right)\left(n_{0}+m+1\right)} .
$$

This implies

$$
\widetilde{u}_{n_{0}+1} \frac{n_{0}+1}{n_{0}+1+m+1}>\widetilde{u}_{n_{0}} \frac{n_{0}}{n_{0}+m+1} .
$$

Hence, the supremum for $m$ is not attained at $n_{0}$. Therefore $n(m+1)>n(m)=$ $m$, that is, $n(m+1) \geq m+1$. 
Lemma 4.5.41. Let $u \in \mathcal{F} \ell^{\infty, 1}\left(\Gamma^{*}\right)$. Then the supremum corresponding to $u$ by 4.5.38 is decreasing with respect to $m$. This remains true if we replace $\widetilde{u}_{n}$ with any positive sequence converging to zero.

Proof. Let $m \in \mathbb{N}$ and set $n_{0}:=n(m)$ and $n_{1}:=n(m+1)$. By definition of $n(m)$,

$$
\widetilde{u}_{n_{0}} \frac{n_{0}}{n_{0}+m} \geq \widetilde{u}_{n_{1}} \frac{n_{1}}{n_{1}+m} \geq \widetilde{u}_{n_{1}} \frac{n_{1}}{n_{1}+m+1} .
$$

This proves the lemma.

We can now prove

Theorem 4.5.42. There is an open neighbourhood $V$ of $0 \in \mathbb{C}^{2}$ such that for all $u_{0} \in \mathcal{F} \ell^{\infty, 1}\left(\Gamma^{*}\right)$ there is an $N \in \mathbb{N}$ and a $C>0$ with $u_{0} \in B_{C}^{N}$ as well as a $\delta>0$ such that for all $k \in V$ and all $u \in B_{C}^{N}$ the entries of the matrix $\mathcal{A}_{ \pm \nu}\left(k+k_{\nu}^{ \pm}\left(\widehat{u}_{0}\right), u\right)$ are $\ell^{\infty, 1}\left(\Gamma_{\delta}^{*}\right)$ with respect to $\nu$, with a bound which depends only on $u$. In particular, the perturbed Fourier transform $\widehat{u}(u, \pm \nu)$ is $\ell^{\infty, 1}\left(\Gamma_{\delta}^{*}\right)$.

Proof. Through lemma 4.5.33, we have already seen that $\mathcal{A}_{ \pm \nu}\left(k+k_{\nu}^{ \pm}\left(\widehat{u}_{0}\right), u\right)$ is $c^{0}\left(\Gamma_{\delta}^{*}\right)$ with respect to $\nu$, so this result merely needs to be refined. To this end, we make the expansion

$$
\begin{aligned}
& \bar{u}\left(1-\left(\Delta_{k+k_{\nu}^{ \pm}\left(\widehat{u}_{0}\right)}-4 \pi^{2} \widehat{u}_{0}\right)^{-1}\left(1-\pi_{K_{ \pm \nu}}\right) \bar{u}\right)^{-1} \\
&=\bar{u}+\bar{u}\left(\Delta_{k+k_{\nu}^{ \pm}\left(\widehat{u}_{0}\right)}-4 \pi^{2} \widehat{u}_{0}\right)^{-1} \\
&\left.\cdot\left(1-\pi_{K_{ \pm \nu}}\right) \bar{u}\left(1-\Delta_{k+k_{\nu}^{ \pm}\left(\widehat{u}_{0}\right)}-4 \pi^{2} \widehat{u}_{0}\right)^{-1}\left(1-\pi_{K_{ \pm \nu}}\right) \bar{u}\right)^{-1}
\end{aligned}
$$

according to lemma 4.5.23. For the restriction to $K_{ \pm \nu}$, we need to examine the Fourier transform of this expression at $\kappa=0$ and $\kappa= \pm \nu$ with respect to $\nu$. For the first term, we simply get

$$
\left(\begin{array}{cc}
\widehat{\bar{u}}(0) & \widehat{\bar{u}}( \pm \nu) \\
\widehat{\bar{u}}(\mp \nu) & \widehat{\bar{u}}(0)
\end{array}\right)=\left(\begin{array}{cc}
0 & \widehat{u}( \pm \nu) \\
\widehat{u}(\mp \nu) & 0
\end{array}\right)
$$

which is certainly $\ell^{\infty, 1}\left(\Gamma_{\delta}^{*}\right)$ because $\widehat{u} \in \ell^{\infty, 1}\left(\Gamma^{*}\right)$. For the second term, we get the Fourier transform

$$
\sum_{\rho \in \Gamma^{*}} \widehat{\bar{u}}(\kappa-\rho) g(k, \nu, \rho) f(k, \nu, \rho)
$$

where $f$ is the Fourier transform of $\bar{u}\left(1-\left(\Delta_{k+k_{\nu}^{ \pm}\left(\widehat{u}_{0}\right)}-4 \pi^{2} \widehat{u}_{0}\right)^{-1}\left(1-\pi_{K_{ \pm \nu}}\right) \bar{u}\right)^{-1}$. Now

$$
\left|\sum_{\rho \in \Gamma^{*}} \widehat{\bar{u}}(\kappa-\rho) g(k, \nu, \rho) f(k, \nu, \rho)\right| \leq \sum_{\rho \in \Gamma^{*}}|\widehat{u}(\kappa-\rho) g(k, \nu, \rho) f(k, \nu, \rho)| .
$$

It is therefore sufficient to prove that

$$
(\nu, \rho) \mapsto \widehat{u}(\kappa-\rho) g(k, \nu, \rho) f(k, \nu, \rho)
$$


is in $\ell^{\infty, 1}\left(\Gamma_{\delta}^{*}\right) \widehat{\otimes} \ell^{1}\left(\Gamma^{*}\right)$ for $\kappa=0$ and $\kappa= \pm \nu$. By the Hardy-Littlewood inequality, theorem 2.3.6. and then Hardy's lemma, proposition 2.3.5, and the Hardy-Littlewood inequality, we have

$$
\sum_{\rho \in \Gamma^{*}}|\widehat{u}(\kappa-\rho) g(k, \nu, \rho) f(k, \nu, \rho)| \leq \sum_{n=1}^{\infty} \widehat{u}^{\star}(n) g^{\star}(k, \nu, n) f^{\star}(k, \nu, n),
$$

(where $f^{\star}$ is the decreasing rearrangement of $f$ with respect to $\rho$ ) because the decreasing rearrangement of $\widehat{u}$ is invariant under translations. We can estimate this further by

$$
\sum_{n=1}^{\infty} \widehat{u}^{\star}(n) g^{\star}(k, \nu, n) f^{\star}(k, \nu, n) \leq\left(\sup _{n \in \mathbb{N}} \widehat{u}^{\star}(n) g^{\star}(k, \nu, n) n\right) \sum_{n=1}^{\infty} \frac{f^{\star}(k, \nu, n)}{n} .
$$

Since $f(k, \cdot, \cdot) \in c^{0}\left(\Gamma_{\delta}^{*}\right) \widehat{\otimes} \ell^{\infty, 1}\left(\Gamma^{*}\right)$, the sum is bounded by a constant independent of $\nu$ (and also independent of $k$ ). What we need to show now is

$$
\sum_{m=1}^{\infty} \frac{1}{m}\left(\nu \mapsto \sup _{n \in \mathbb{N}} \widehat{u}^{\star}(n) g^{\star}(k, \nu, n) n\right)^{\star}(m)<\infty .
$$

By monotonicity, the considerations in definition 4.5.37 and lemma 4.5.41, it is sufficient to show

$$
\sum_{m=1}^{\infty} \frac{1}{m} \widetilde{u}_{n(m)} \frac{n(m)}{n(m)+m}<\infty .
$$

Due to lemma 4.5.40, we can estimate this series as follows:

$$
\sum_{m=1}^{\infty} \frac{1}{m} \widetilde{u}_{n(m)} \frac{n(m)}{n(m)+m} \leq \sum_{m=1}^{\infty} \frac{\widetilde{u}_{n(m)}}{m} \leq \sum_{m=1}^{\infty} \frac{\widetilde{u}_{m}}{m} \leq \frac{4}{1-\frac{1}{\sqrt{2}}}\|u\|_{\mathcal{F} \infty, 1}<\infty
$$

due to proposition 4.5 .35

Thus, we have shown that the entries of $\mathcal{A}_{ \pm \nu}\left(k+k_{\nu}^{ \pm}\left(\widehat{u}_{0}\right), u\right)$ are $\ell^{\infty, 1}\left(\Gamma_{\delta}^{*}\right)$ uniformly for all fixed $k \in V$. In order to establish the same for $\widehat{u}(u, \pm \nu)$, it is, by definition 4.5.31. sufficient to prove that the entries of $\mathcal{A}_{ \pm \nu}\left(k_{ \pm \nu}+k_{\nu}^{ \pm}\left(\widehat{u}_{0}\right), u\right)$ are $\ell^{\infty, 1}\left(\Gamma_{\delta}^{*}\right)$. Let $\|\cdot\|$ denote the matrix norm, then we have

$$
\begin{aligned}
\| \mathcal{A}_{ \pm \nu} & \left(k_{ \pm \nu}+k_{\nu}^{ \pm}\left(\widehat{u}_{0}\right), u\right) \| \\
= & \left\|\mathcal{A}_{ \pm \nu}\left(k_{\nu}^{ \pm}\left(\widehat{u}_{0}\right), u\right)+\mathcal{A}_{ \pm \nu}\left(k_{ \pm \nu}+k_{\nu}^{ \pm}\left(\widehat{u}_{0}\right), u\right)-\mathcal{A}_{ \pm \nu}\left(k_{\nu}^{ \pm}\left(\widehat{u}_{0}\right), u\right)\right\| \\
& \leq\left\|\mathcal{A}_{ \pm \nu}\left(k_{\nu}^{ \pm}\left(\widehat{u}_{0}\right), u\right)\right\|+\left\|\mathcal{A}_{ \pm \nu}\left(k_{ \pm \nu}+k_{\nu}^{ \pm}\left(\widehat{u}_{0}\right), u\right)-\mathcal{A}_{ \pm \nu}\left(k_{\nu}^{ \pm}\left(\widehat{u}_{0}\right), u\right)\right\| .
\end{aligned}
$$

The first summand is $\ell^{\infty, 1}\left(\Gamma_{\delta}^{*}\right)$ by what we have just proven. We can estimate the second summand using the mean value theorem:

$$
\begin{aligned}
\left\|\mathcal{A}_{ \pm \nu}\left(k_{ \pm \nu}+k_{\nu}^{ \pm}\left(\widehat{u}_{0}\right), u\right)-\mathcal{A}_{ \pm \nu}\left(k_{\nu}^{ \pm}\left(\widehat{u}_{0}\right), u\right)\right\| & \\
\quad & \quad \sup _{k \in V}\left\|\frac{\partial}{\partial k} \mathcal{A}_{ \pm \nu}\left(k+k_{\nu}^{ \pm}\left(\widehat{u}_{0}\right), u\right)\right\|\left\|k_{ \pm \nu}\right\| .
\end{aligned}
$$

By lemma 4.5.21, the supremum is bounded with respect to $\nu$. Hence, in order to finish the proof, we must merely show that $\left(\left\|k_{ \pm \nu}\right\|\right)_{\nu \in \Gamma_{\delta}^{*}} \in \ell^{\infty, 1}\left(\Gamma_{\delta}^{*}\right)$. We do 
so by performing a simple analysis of the diagonal entries of the matrix 4.5.20]. For this purpose, define

$$
K(k):=-4 \pi^{2}\left(\left(k+k_{\nu}^{ \pm}\left(\widehat{u}_{0}\right)\right)^{2}+\widehat{u}_{0},\left(k+k_{\nu}^{\mp}\left(\widehat{u}_{0}\right)\right)^{2}+\widehat{u}_{0}\right)
$$

and let $D(k)$ be the two-component vector of the diagonal entries of $\mathcal{A}_{ \pm \nu}(k+$ $\left.k_{\nu}^{ \pm}\left(\widehat{u}_{0}\right), u\right)$. Denote the derivatives with respect to $k$ by a prime. Then we have

$$
\begin{aligned}
0=K(0) & =K\left(k_{ \pm \nu}\right)-K^{\prime}\left(k_{ \pm \nu}\right)\left(k_{ \pm \nu}\right)+o\left(\left\|k_{ \pm \nu}\right\|\right), \\
D(0) & =D\left(k_{ \pm \nu}\right)-D^{\prime}\left(k_{ \pm \nu}\right)\left(k_{ \pm \nu}\right)+o\left(\left\|k_{ \pm \nu}\right\|\right) .
\end{aligned}
$$

By definition 4.5.31. $K\left(k_{ \pm \nu}\right)+D\left(k_{ \pm \nu}\right)=0$, so adding these two equations yields

$$
D(0)=-\left(\left(K^{\prime}+D^{\prime}\right)\left(k_{ \pm \nu}\right)\right)\left(k_{ \pm \nu}\right)+o\left(\left\|k_{ \pm \nu}\right\|\right) .
$$

From the proof of proposition 4.5.29, we already know that $k_{ \pm \nu}$ vanishes for large $\|\nu\|$. Lemma 4.5 .21 implies that $D^{\prime}\left(k_{ \pm \nu}\right)$ vanishes for large $\|\nu\|$. Now, we have

$$
\frac{\partial}{\partial k} K(k)=-4 \pi^{2}\left(\begin{array}{cc}
2 k_{1}-\nu_{1}+i \nu_{2} \xi\left(\widehat{u}_{0}, \nu\right) & 2 k_{2}-i \nu_{1} \xi\left(\widehat{u}_{0}, \nu\right)-\nu_{2} \\
2 k_{1}+\nu_{1}+i \nu_{2} \xi\left(\widehat{u}_{0}, \nu\right) & 2 k_{2}-i \nu_{1} \xi\left(\widehat{u}_{0}, \nu\right)+\nu_{2}
\end{array}\right),
$$

from which we can calculate

$$
\operatorname{det} K^{\prime}\left(k_{ \pm \nu}\right)=32 \pi^{4}\left(i \xi\left(\widehat{u}_{0}, \nu\right)\|\nu\|^{2}+2 k_{ \pm \nu, 1} \nu_{2}-2 k_{ \pm \nu, 2} \nu_{1}\right),
$$

an expression bounded away from zero for large $\|\nu\|$. Hence, $\left(K^{\prime}+D^{\prime}\right)\left(k_{ \pm \nu}\right)$ is invertible with a uniformly bounded inverse for all sufficiently large $\|\nu\|$. It follows that

$$
-\left(\left(K^{\prime}+D^{\prime}\right)\left(k_{ \pm \nu}\right)\right)^{-1} D(0)=k_{ \pm \nu}+\left(\left(K^{\prime}+D^{\prime}\right)\left(k_{ \pm \nu}\right)\right)^{-1} o\left(\left\|k_{ \pm \nu}\right\|\right) .
$$

Since the entries of $D(0)$ are in $\ell^{\infty, 1}\left(\Gamma_{\delta}^{*}\right)$, the same must now be true of $k_{ \pm \nu}$, concluding the proof of the theorem.

Note that by considering the inverse of $K^{\prime}\left(k_{ \pm \nu}\right)$ in the above proof, we are actually able to prove much better regularity results for $k_{ \pm \nu}$ in certain situations. We shall investigate this in more detail in subsection 4.5.3.

This theorem also extends to variations of the perturbed Fourier transform with respect to $u$ :

Lemma 4.5.45. For all $u \in \mathcal{F} \ell^{\infty, 1}\left(\Gamma^{*}\right)$ there is a $\delta>0$ such that the variation of the perturbed Fourier transform of $u$ with respect to $u$ is a bounded operator mapping functions from $\mathcal{F} \ell^{\infty, 1}\left(\Gamma^{*}\right)$ to sequences in $\ell^{\infty, 1}\left(\Gamma_{\delta}^{*}\right)$.

Proof. We use the $A, B$ and $\mathcal{C}$ notation from the proof of theorem 4.5.25. As the proof of that theorem shows, we need to investigate two summands:

$$
\begin{aligned}
& \left.h \mapsto \pi_{K_{ \pm \nu}}(1-A B)^{-1} \bar{h}(1-B A)^{-1}\right|_{K_{ \pm \nu}}, \\
& \left.h \mapsto \pi_{K_{ \pm \nu}}(1-A B)^{-1} A B \mathcal{C}(h) B A(1-B A)^{-1}\right|_{K_{ \pm \nu}},
\end{aligned}
$$

where we write $\bar{h}$ for $\left.\left(1-\pi_{K_{0}}\right) h\right|_{K_{0}^{\perp}}$. Let's begin with the first summand. Due to lemma 4.5.23, we have the expansion

$$
\begin{aligned}
(1-A B)^{-1} & \bar{h}(1-B A)^{-1}=\bar{h}+A(1-B A)^{-1} B \bar{h} \\
& +\bar{h} B A(1-B A)^{-1}+A(1-B A)^{-1} B \bar{h} B A(1-B A)^{-1},
\end{aligned}
$$


the Fourier transform of which we must study for $\kappa=0$ and $\kappa= \pm \nu$. The analysis is very similar to the proof of theorem 4.5.42. The first term yields $\ell^{\infty, 1}\left(\Gamma^{*}\right)$-sequences analogous to 4.5 .43 because $\bar{h}(0)=0$. For the second term we get (again, we let $f$ denote the $A(1-B A)^{-1}$-part)

$$
\sum_{\rho \in \Gamma^{*}} \widehat{\bar{h}}(\rho) g(k, \nu, \rho) f(k, \nu, \kappa-\rho),
$$

whose $\ell^{\infty, 1}\left(\Gamma^{*}\right)$-norm with respect to $\nu$ is bounded by $C\|h\|_{\mathcal{F} \infty, 1}$ with some $C>$ 0 independent of $k$ by an analogous estimate as for 4.5.44) (the fact that the $\kappa$-dependency is in $f$ here does not matter due to the Hardy-Littlewood inequality). For the third term we get

$$
\sum_{\rho \in \Gamma^{*}} \widehat{\bar{h}}(\kappa-\rho) g(k, \nu, \rho) f(k, \nu, \rho),
$$

which can be estimated exactly as 4.5.44. The fourth and final term looks a bit more complicated but is conceptually no more difficult. We get

$$
\sum_{\rho_{1} \in \Gamma^{*}} f\left(k, \nu, \kappa-\rho_{1}\right) g\left(k, \nu, \rho_{1}\right) \sum_{\rho_{2} \in \Gamma^{*}} \widehat{\bar{h}}\left(\rho_{1}-\rho_{2}\right) g\left(k, \nu, \rho_{2}\right) f\left(k, \nu, \rho_{2}\right) .
$$

The absolute value of the second sum can be estimated as (4.5.44), yielding as estimate an $\ell^{\infty, 1}\left(\Gamma^{*}\right)$-sequence $h^{\prime}$ whose norm is bounded by $C\|h\|_{\mathcal{F} \infty, 1}$, in particular independently of $\rho_{1}$, so that we can estimate the absolute value of 4.5.48 against

$$
\sum_{\rho_{1} \in \Gamma^{*}}\left|f\left(k, \nu, \kappa-\rho_{1}\right) g\left(k, \nu, \rho_{1}\right) h^{\prime}(\nu)\right|=\left|h^{\prime}(\nu)\right| \sum_{\rho_{1} \in \Gamma^{*}}\left|f\left(k, \nu, \kappa-\rho_{1}\right) g\left(k, \nu, \rho_{1}\right)\right| .
$$

The sum over $\rho_{1}$ of an $\ell^{\infty, 1}\left(\Gamma^{*}\right) \cdot \ell^{1, \infty}\left(\Gamma^{*}\right)$-product is clearly bounded with respect to $k$ and $\nu$ in this expression.

This shows the sought property for the first expression in 4.5.46). The second expression we need not expand, as by lemma 4.5.23, we can rewrite it like

$$
(1-A B)^{-1} A B C(h) B A(1-B A)^{-1}=A(1-B A)^{-1} B \mathcal{C}(h) B A(1-B A)^{-1} .
$$

Given that $\mathcal{C}$ boundedly maps $\ell^{\infty, 1}\left(\Gamma^{*}\right)$-left multiplications to $\ell^{\infty, 1}\left(\Gamma^{*}\right)$-left multiplications, we can estimate this expression just as (4.5.48).

In total, we have shown the sought property for the variation of $\mathcal{A}_{ \pm \nu}(k+$ $\left.k_{\nu}^{ \pm}\left(\widehat{u}_{0}\right), \cdot\right)$ uniformly for fixed $k \in V$. To prove the theorem, we now need to show the same for $k=k_{ \pm}$. In the proof of theorem 4.5.42 we have already shown that $\left\|k_{ \pm}\right\| \in \ell^{\infty, 1}\left(\Gamma_{\delta}^{*}\right)$, so once again, we can use the mean value theorem to finish the proof. It only remains to show that the $k$-derivative of 4.5.27) is uniformly bounded with respect to $\nu$. But looking at 4.5.28, we see that the $k$-derivatives of the individual factors of the variation of $\mathcal{A}_{ \pm \nu}\left(k+k_{\nu}^{ \pm}\left(\widehat{u}_{0}\right), \cdot\right)$ are all bounded with respect to $\nu$, just as the factors themselves. Hence, the statement follows. 
Lemma 4.5.49. The mapping

$$
\begin{aligned}
\ell^{\infty, 1}\left(\Gamma^{*}\right) \stackrel{\mathcal{F}^{\prime}}{\longrightarrow} \mathcal{F} \ell^{\infty, 1}\left(\Gamma^{*}\right) \longrightarrow & \longrightarrow \ell^{\infty, 1}\left(\Gamma_{\delta}^{*}\right), \\
\widehat{u} \longmapsto & \longrightarrow(\widehat{u}(u, \nu))_{\nu \in \Gamma_{\delta}^{*}}
\end{aligned}
$$

with sufficiently small $\delta>0$ depending on $u$, is locally differentiable in $u$. Its derivatives are continuous linear operators from $\ell^{\infty, 1}\left(\Gamma^{*}\right)$ to $\ell^{\infty, 1}\left(\Gamma_{\delta}^{*}\right)$ whose natural restriction to $\ell^{\infty, 1}\left(\Gamma_{\delta}^{*}\right)$ is boundedly invertible.

Proof. By theorem 4.5.42 the mapping (4.5.50) exists and is continuous (due to the $u$-dependence of $\delta$, it is defined only locally). By lemma 4.5.45 and since the Fourier transform is an isometry on Fourier spaces, the mapping is, for sufficiently small $\delta>0$, differentiable in $u$, its derivatives being continuous linear operators from $\ell^{\infty, 1}\left(\Gamma^{*}\right)$ to $\ell^{\infty, 1}\left(\Gamma_{\delta}^{*}\right)$. It remains to show that these operators have boundedly invertible natural restrictions for sufficiently small $\delta>0$. To this end, we once more investigate the two summands 4.5.46). The norm of the second summand vanishes as $\delta \rightarrow 0$ due to lemma 4.5.13 For the norm of the first summand, we use lemma 4.5.23 to rewrite the expansion 4.5.47) as

$$
\begin{aligned}
& (1-A B)^{-1} \bar{h}(1-B A)^{-1}=\bar{h}+(1-A B)^{-1} A B \bar{h} \\
& +\bar{h} B A(1-B A)^{-1}+A(1-B A)^{-1} B \bar{h} B A(1-B A)^{-1} .
\end{aligned}
$$

Again, due to lemma 4.5.13, the last three terms of this expansion vanish as $\delta \rightarrow$ 0 . The restriction of the Fourier transform of the first term, on the other hand, is clearly boundedly invertible. Hence, the claim follows by continuity.

Corollary 4.5.51. Let $u \in \mathcal{F} \ell^{\infty, 1}\left(\Gamma^{*}\right)$. Then there is a sequence $\left(u_{n}\right)_{n \in \mathbb{N}}$ of finite-type potentials in $\mathcal{F} \ell^{\infty, 1}\left(\Gamma^{*}\right)$ converging to $u$. In other words, the finitetype potentials are dense in $\mathcal{F} \ell^{\infty, 1}\left(\Gamma^{*}\right)$.

Proof. Since we want to solve a local question, we can make use of the locally defined map 4.5.50). The set of $\Gamma_{\delta}^{*}$-sequences all but a finite number of whose elements are zero are dense in $\ell^{\infty, 1}\left(\Gamma_{\delta}^{*}\right)$. Hence, there is a sequence $\left(f_{n}\right)_{n \in \mathbb{N}}$ of such sequences converging to $\widehat{u}(u, \cdot)$ in the $\ell^{\infty, 1}\left(\Gamma_{\delta}^{*}\right)$-norm. For each $f_{n}$ which is the image of a potential $u$ under the second half of 4.5.50), that potential has finite-type by definition 4.5.32. Now, by lemma 4.5.49, the restriction of the variation of the mapping (4.5.50 with respect to $\widehat{u}$ is invertible. Therefore, there are open neighbourhoods $U_{1}$ and $U_{2}$ of $\widehat{u}$ and $\widehat{u}(u, \cdot)$, respectively, on which 4.5.50 is a local diffeomorphism. All but a finite number of the elements of the sequence $\left(f_{n}\right)_{n \in \mathbb{N}}$ lie in $U_{2}$. Hence, the preimages of these elements yield the desired sequence converging to $u$.

As a final remark, we use the proof of lemma 4.5.45 to refine lemma 4.5.21

Corollary 4.5.52. There is an open neighbourhood $V$ of $0 \in \mathbb{C}^{2}$ which depends only on $\Gamma^{*}$ such that for all $u_{0} \in \mathcal{F} \ell^{\infty, 1}\left(\Gamma^{*}\right)$ there is an $N \in \mathbb{N}$ and a $C>0$ with $u_{0} \in B_{N}^{C}$ as well as a $\delta>0$ such that for all $k \in V$, all $\nu \in \Gamma_{\delta}^{*}$ and all $u \in B_{C}^{N}$, the $k$-derivative of the matrix $\mathcal{A}_{ \pm \nu}\left(k+k_{\nu}^{ \pm}\left(\widehat{u}_{0}\right), u\right)$ consists of bounded linear operators mapping the space $\mathcal{F} \ell^{\infty, 1}\left(\Gamma_{\delta}^{*}\right)$ (with respect to $\nu$ ) into itself. 
Proof. Using the $A, B$ and $\mathcal{C}$ notation from the proof of lemma 4.5.21, and given the fact that both $A B$ and $\mathcal{C} B$ map $\mathcal{F} \ell^{\infty, 1}\left(\Gamma_{\delta}^{*}\right)$ into itself, we can estimate the $k$-derivative of $\mathcal{A}_{ \pm \nu}\left(k+k_{\nu}^{ \pm}\left(\widehat{u}_{0}\right), u\right)$ just as the first equation in 4.5.46.

\subsubsection{Per-double-point approximation of the Fermi curve}

We would like to attach to each Fermi curve a data set which uniquely captures its asymptotic geometric structure. That is, we would like to construct a parameter space such that each element from this space asymptotically describes a Fermi curve, and that two distinct elements from this parameter space describe distinct Fermi curves.

The potential $u$ provides, through its Fourier transform, a countable number of parameters defining the corresponding Fermi curve in its entirety. However, this description of a Fermi curve is not necessarily unique. A space of unique parameters thus can be said to partially solve the moduli problem outlined in section 4.1. We shall therefore call our to-be-constructed parameter space the moduli space. We expect the moduli space to be made up of countably many parameters with equal or better regularity properties than $\ell^{\infty, 1}\left(\Gamma^{*}\right)$.

Our moduli space should be derived strictly from the geometry of the Fermi curve. Due to corollary 4.3.9 and by the results of section 4.4. asymptotically, the only deviation of the Fermi curve from the constant potential Fermi curves occurs near the double points. In theorem 4.5 .19 we established asymptotic double-point local descriptions of the Fermi curve through determinants of $2 \times 2$-matrices. We shall use these descriptions to create simple first-order approximations of the Fermi curve around each double point sufficiently far away from the origin. These approximations are good enough to define a very simple local model of the Fermi curve near each double point which is sufficiently far away from the origin. Setting out from these models, we can then generalise to the actual Fermi curves using standard techniques from real and complex analysis. We also arrive at a geometrically proper definition of a splitting double point for arbitrary potentials.

Let us begin with an approximation of $\mathcal{A}_{ \pm \nu}$ near the double points.

Lemma 4.5.53. There is an open neighbourhood $V$ of $0 \in \mathbb{C}^{2}$ such that for all $u_{0} \in \mathcal{F} \ell^{\infty, 1}\left(\Gamma^{*}\right)$ there is an $N \in \mathbb{N}$ and a $C>0$ with $u_{0} \in B_{C}^{N}$, such that for all $\epsilon>0$ there is $a \delta>0$ and a $2 \times 2$-matrix valued function $r$ such that for all $u \in B_{C}^{N}$ and all $\nu \in \Gamma_{\delta}^{*}$ we have

$$
\begin{gathered}
\left(\begin{array}{cc}
-4 \pi^{2}\left(\left(k+k_{\nu}^{ \pm}\left(\widehat{u}_{0}\right)\right)^{2}+\widehat{u}_{0}\right) & 0 \\
0 & -4 \pi^{2}\left(\left(k+k_{\nu}^{\mp}\left(\widehat{u}_{0}\right)\right)^{2}+\widehat{u}_{0}\right)
\end{array}\right) \\
+\mathcal{A}_{ \pm \nu}\left(k+k_{\nu}^{ \pm}\left(\widehat{u}_{0}\right), u\right) \\
=-4 \pi^{2}\left(\begin{array}{cc}
2\left(k-k_{ \pm \nu} \mid k_{\nu}^{ \pm}\left(\widehat{u}_{0}\right)\right) & 0 \\
0 & 2\left(k-k_{ \pm \nu} \mid k_{\nu}^{\mp}\left(\widehat{u}_{0}\right)\right)
\end{array}\right) \\
\quad+\left(\begin{array}{cc}
0 & \widehat{u}(u, \pm \nu) \\
\widehat{u}(u, \mp \nu) & 0
\end{array}\right)+r\left(k-k_{ \pm \nu}\right),
\end{gathered}
$$

with $k_{ \pm \nu}$ as in proposition 4.5.29, $k \in V$ with $\left\|k-k_{ \pm \nu}\right\|<\delta$ and $\left\|r\left(k-k_{ \pm \nu}\right)\right\| \leq$ $\epsilon\left\|k-k_{ \pm \nu}\right\|$. 
Proof. By lemma 4.5.21, there is a suitable $V$ such that $\mathcal{A}_{ \pm \nu}\left(k+k_{\nu}^{ \pm}\left(\widehat{u}_{0}\right), u\right)$ is continuously differentiable in $k=k_{ \pm \nu}$. We then have by definition 4.5.31

$$
\begin{aligned}
& \left(\begin{array}{cc}
-4 \pi^{2}\left(\left(k+k_{\nu}^{ \pm}\left(\widehat{u}_{0}\right)\right)^{2}+\widehat{u}_{0}\right) & 0 \\
0 & -4 \pi^{2}\left(\left(k+k_{\nu}^{\mp}\left(\widehat{u}_{0}\right)\right)^{2}+\widehat{u}_{0}\right)
\end{array}\right) \\
& +\mathcal{A}_{ \pm \nu}\left(k+k_{\nu}^{ \pm}\left(\widehat{u}_{0}\right), u\right) \\
& =\left(\begin{array}{cc}
-4 \pi^{2}\left(\left(k+k_{\nu}^{ \pm}\left(\widehat{u}_{0}\right)\right)^{2}+\widehat{u}_{0}\right) & 0 \\
0 & -4 \pi^{2}\left(\left(k+k_{\nu}^{\mp}\left(\widehat{u}_{0}\right)\right)^{2}+\widehat{u}_{0}\right)
\end{array}\right) \\
& -\left(\begin{array}{cc}
-4 \pi^{2}\left(\left(k_{ \pm \nu}+k_{\nu}^{ \pm}\left(\widehat{u}_{0}\right)\right)^{2}+\widehat{u}_{0}\right) & 0 \\
0 & -4 \pi^{2}\left(\left(k_{ \pm \nu}+k_{\nu}^{\mp}\left(\widehat{u}_{0}\right)\right)^{2}+\widehat{u}_{0}\right)
\end{array}\right) \\
& +\left(\begin{array}{cc}
-4 \pi^{2}\left(\left(k_{ \pm \nu}+k_{\nu}^{ \pm}\left(\widehat{u}_{0}\right)\right)^{2}+\widehat{u}_{0}\right) & 0 \\
0 & -4 \pi^{2}\left(\left(k_{ \pm \nu}+k_{\nu}^{\mp}\left(\widehat{u}_{0}\right)\right)^{2}+\widehat{u}_{0}\right)
\end{array}\right) \\
& +\mathcal{A}_{ \pm \nu}\left(k_{ \pm \nu}+k_{\nu}^{ \pm}\left(\widehat{u}_{0}\right), u\right)+\left(\frac{\partial}{\partial k} \mathcal{A}_{ \pm \nu}\left(k_{ \pm \nu}+k_{\nu}^{ \pm}\left(\widehat{u}_{0}\right), u\right)\right)\left(k-k_{ \pm \nu}\right) \\
& +O\left(\left\|k-k_{ \pm \nu}\right\|^{2}\right) \\
& =-4 \pi^{2}\left(\begin{array}{cc}
2\left(k-k_{ \pm \nu} \mid k_{\nu}^{ \pm}\left(\widehat{u}_{0}\right)\right) & 0 \\
0 & 2\left(k-k_{ \pm \nu} \mid k_{\nu}^{\mp}\left(\widehat{u}_{0}\right)\right)
\end{array}\right) \\
& +\left(\begin{array}{cc}
0 & \widehat{u}(u, \pm \nu) \\
\widehat{u}(u, \mp \nu) & 0
\end{array}\right)+\left(\frac{\partial}{\partial k} \mathcal{A}_{ \pm \nu}\left(k_{ \pm \nu}+k_{\nu}^{ \pm}\left(\widehat{u}_{0}\right), u\right)\right)\left(k-k_{ \pm \nu}\right) \\
& -4 \pi^{2}\left(\begin{array}{cc}
k^{2}-k_{ \pm \nu}^{2} & 0 \\
0 & k^{2}-k_{ \pm \nu}^{2}
\end{array}\right)+O\left(\left\|k-k_{ \pm \nu}\right\|^{2}\right) .
\end{aligned}
$$

Now, by lemma 4.5.21, the norm of the $k$-derivative of $\mathcal{A}_{ \pm \nu}$ vanishes for $\|\nu\| \rightarrow$ $\infty$, so for sufficiently small $\delta$, in combination with an application of the CauchySchwarz inequality to $k^{2}-k_{ \pm \nu}^{2}=\left(k+k_{ \pm \nu} \mid k-k_{ \pm \nu}\right)$, we can combine the last three terms to a function $r$ satisfying the statement of the lemma.

This lemma shows that, by taking the determinant, the Fermi curve near the double point associated with $\nu$ is asymptotically approximated by the equation

$$
\left(k-k_{ \pm \nu} \mid 2 k_{\nu}^{+}\left(\widehat{u}_{0}\right)\right)\left(k-k_{ \pm \nu} \mid 2 k_{\nu}^{-}\left(\widehat{u}_{0}\right)\right)=\frac{\widehat{u}(u, \nu) \widehat{u}(u,-\nu)}{-16 \pi^{4}} .
$$

(In the present case, we might actually perform the shift $k \mapsto k+k_{ \pm \nu}$ to get rid of $k_{ \pm \nu}$, but this won't be possible with the actual Fermi curve, so we leave it in.) That is, equation (4.5.54 is sufficient to distinguish two asymptotically distinct Fermi curves from each other. On the other hand, it is not clear whether this equation does not lead to unwanted distinction in cases where two distinct potentials have asymptotically identical Fermi curves. Nevertheless, we shall take this equation as a simple model of the Fermi curve around a single double point. Let us now define our model moduli, the prototypes of the real moduli we intend to define later.

Definition 4.5.55. Let $\left(k_{1}, k_{2}\right)$ be the canonical coordinates of $\mathbb{C}^{2}$. The simplified model of the Fermi curve near the double point associated with $\nu \in \Gamma_{\delta}^{*}$, with suitable $\delta>0$, defines a relation between $k_{1}$ and $k_{2}$. Somewhat arbitrarily, we define the model modulus of the Fermi curve with respect to $\nu$ as

$$
\widetilde{t}(\nu):= \begin{cases}0 & \text { if } \widehat{u}(u, \nu)=0 \text { or } \widehat{u}(u,-\nu)=0, \\ 16 \pi^{3} \oint k_{1} \mathrm{~d} k_{2} & \text { otherwise. }\end{cases}
$$


In the second case, the contour to be integrated over is a closed path on the Fermi curve model which encompasses the model handle, in positive direction, with a winding number of one (in what follows, the details will become clear).

It is, at this point, not clear whether such a definition actually makes sense: we have to make sure that the prescribed contour actually exists if neither $\widehat{u}(u, \nu)=0$ nor $\widehat{u}(u,-\nu)=0$, and that the integral of the form $k_{1} \mathrm{~d} k_{2}$ is well-defined. In any case, for our model by its inherent structure, and for the Fermi curve it is based on by theorem 4.1.3 the dependence of $k_{1}$ on $k_{2}$ is clearly holomorphic outside a discrete set, and the contour integral, if it exists, is capable of capturing the asymptotic deviation of the Fermi curve to a constant-potential Fermi curve. Yet, even for a well-defined collection of moduli $(\widetilde{t}(\nu))_{\nu \in \Gamma_{\delta}^{*}}$, we still need to make sure they belong to a structure which sensibly describes the available parameter space.

Theorem 4.5.56. For all $u \in \mathcal{F} \ell^{\infty, 1}\left(\Gamma^{*}\right)$ there is a $\delta>0$ such that the corresponding model moduli $\widetilde{t}(\nu)$ are well-defined for all $\nu \in \Gamma_{\delta}^{*}$, with $\left.\widetilde{t}(\nu)\right)_{\nu \in \Gamma_{\delta}^{*}} \in$ $\ell^{1}\left(\Gamma_{\delta}^{*}\right)$. More precisely, $(\widetilde{t}(\nu))_{\nu \in \Gamma_{\delta}^{*}}$ belongs to the second-order Bessel potential space of the quasi-Banach space $\ell^{\infty, 1 / 2}\left(\Gamma_{\delta}^{*}\right)_{e}$, the even part of the quasi-Banach space $\ell^{\infty, 1 / 2}\left(\Gamma_{\delta}^{*}\right)$, and the mapping $u \mapsto \widetilde{t}$ is locally onto.

Proof. Expanding equation (4.5.54 yields

$$
\begin{gathered}
\left(\left(k_{1}-k_{ \pm \nu, 1}\right)\left(-\nu_{1}+i \nu_{2} \xi\left(\widehat{u}_{0}, \nu\right)\right)+\left(k_{2}-k_{ \pm \nu, 2}\right)\left(-i \nu_{1} \xi\left(\widehat{u}_{0}, \nu\right)-\nu_{2}\right)\right) \\
\cdot\left(\left(k_{1}-k_{ \pm \nu, 1}\right)\left(\nu_{1}+i \nu_{2} \xi\left(\widehat{u}_{0}, \nu\right)\right)+\left(k_{2}-k_{ \pm \nu, 2}\right)\left(-i \nu_{1} \xi\left(\widehat{u}_{0}, \nu\right)+\nu_{2}\right)\right) \\
=\frac{\widehat{u}(u, \nu) \widehat{u}(u,-\nu)}{-16 \pi^{4}} .
\end{gathered}
$$

Consider the following linear coordinate transformation:

$$
\begin{aligned}
& \widetilde{a}_{1}:=i\left(k_{1}-k_{ \pm \nu, 1}\right) \nu_{2} \xi\left(\widehat{u}_{0}, \nu\right)-i\left(k_{2}-k_{ \pm \nu, 2}\right) \nu_{1} \xi\left(\widehat{u}_{0}, \nu\right), \\
& \widetilde{a}_{2}:=i\left(k_{1}-k_{ \pm \nu, 1}\right) \nu_{1}+i\left(k_{2}-k_{ \pm \nu, 2}\right) \nu_{2} .
\end{aligned}
$$

The corresponding reverse transformation is

$$
\begin{aligned}
& k_{1}-k_{ \pm \nu, 1}=\frac{1}{i\|\nu\|^{2} \xi\left(\widehat{u}_{0}, \nu\right)}\left(\widetilde{a}_{1} \nu_{2}+\widetilde{a}_{2} \nu_{1} \xi\left(\widehat{u}_{0}, \nu\right)\right), \\
& k_{2}-k_{ \pm \nu, 2}=\frac{1}{i\|\nu\|^{2} \xi\left(\widehat{u}_{0}, \nu\right)}\left(-\widetilde{a}_{1} \nu_{1}+\widetilde{a}_{2} \nu_{2} \xi\left(\widehat{u}_{0}, \nu\right)\right) .
\end{aligned}
$$

With the new coordinates $\widetilde{a}_{1}, \widetilde{a}_{2}$, equation (4.5.57) simplifies to

$$
\left(\widetilde{a}_{1}+i \widetilde{a}_{2}\right)\left(\widetilde{a}_{1}-i \widetilde{a}_{2}\right)=\frac{\widehat{u}(u, \nu) \widehat{u}(u,-\nu)}{-16 \pi^{4}} .
$$

Setting $\widetilde{z}:=\widetilde{a}_{1}+i \widetilde{a}_{2}$ now yields the two equations

$$
\widetilde{z}\left(\widetilde{z}-2 i \widetilde{a}_{2}\right)=\frac{\widehat{u}(u, \nu) \widehat{u}(u,-\nu)}{-16 \pi^{4}}, \quad \widetilde{z}\left(2 \widetilde{a}_{1}-\widetilde{z}\right)=\frac{\widehat{u}(u, \nu) \widehat{u}(u,-\nu)}{-16 \pi^{4}},
$$

giving

$$
\widetilde{a}_{2}=\frac{\widetilde{z}}{2 i}-\frac{\widehat{u}(u, \nu) \widehat{u}(u,-\nu)}{-32 \pi^{4} i \widetilde{z}}, \quad \widetilde{a}_{1}=\frac{\widetilde{z}}{2}+\frac{\widehat{u}(u, \nu) \widehat{u}(u,-\nu)}{-32 \pi^{4} \widetilde{z}}
$$


These last two equations are just reformulations of 4.5.54). This shows that in the case $\widehat{u}(u, \nu) \widehat{u}(u,-\nu) \neq 0, \widetilde{z}$ is a holomorphic coordinate for our Fermi curve model defined on $\mathbb{C} \backslash\{0\}$. Hence, for our contour integral, we can integrate along any circle $\widetilde{z}=c \exp (i \varphi), \varphi \in[0,2 \pi)$ with $c>0$, or indeed, $c \in \mathbb{C} \backslash\{0\}$ because a nonzero argument merely causes a rotation an thus maps a circle into itself. Since $\left(\widetilde{a}_{1}+i \widetilde{a}_{2}\right)\left(\widetilde{a}_{1}-i \widetilde{a}_{2}\right)=\widetilde{a}_{1}^{2}+\widetilde{a}_{2}^{2}$, we may choose

$$
\tilde{a}_{1}=\sqrt{c} \cos \varphi, \quad \tilde{a}_{2}=\sqrt{c} \sin \varphi
$$

by setting

$$
c=\frac{\widehat{u}(u, \nu) \widehat{u}(u,-\nu)}{-16 \pi^{4}} .
$$

Which branch of $\sqrt{c}$ is chosen is immaterial as long as the choice is consistent. With the matter of the contour settled, we shall now calculate the integrals. Firstly note that $\oint \mathrm{d} k_{2}=\oint \mathrm{d}\left(k_{2}-k_{ \pm \nu, 2}\right)=0$ because

$$
\int_{\varphi=0}^{2 \pi} \mathrm{d} \cos \varphi=0, \quad \int_{\varphi=0}^{2 \pi} \mathrm{d} \sin \varphi=0 .
$$

Therefore, the contour integral of $k_{1} \mathrm{~d} k_{2}$ does not depend on a branch choice. Hence, the model moduli $\widetilde{t}(\nu)$ are well-defined. Their value is

$$
\begin{aligned}
\widetilde{t}(\nu)= & 16 \pi^{3} \oint k_{1} \mathrm{~d} k_{2}=16 \pi^{3} \oint k_{1} \mathrm{~d}\left(k_{2}-k_{ \pm \nu, 2}\right) \\
= & 16 \pi^{3}(\oint\left(k_{1}-k_{ \pm \nu, 1}\right) \mathrm{d}\left(k_{2}-k_{ \pm \nu, 2}\right)+\underbrace{\oint k_{ \pm \nu, 1} \mathrm{~d}\left(k_{2}-k_{ \pm \nu, 2}\right)}_{=0}) \\
= & -\frac{16 \pi^{3}}{\|\nu\|^{4} \xi\left(\widehat{u}_{0}, \nu\right)^{2}} \oint\left(\widetilde{a}_{1} \nu_{2}+\widetilde{a}_{2} \nu_{1} \xi\left(\widehat{u}_{0}, \nu\right)\right) \mathrm{d}\left(-\widetilde{a}_{1} \nu_{1}+\widetilde{a}_{2} \nu_{2} \xi\left(\widehat{u}_{0}, \nu\right)\right) \\
= & -\frac{16 \pi^{3}}{\|\nu\|^{4} \xi\left(\widehat{u}_{0}, \nu\right)^{2}}\left(-\nu_{1} \nu_{2} \oint \widetilde{a}_{1} \mathrm{~d} \widetilde{a}_{1}+\nu_{2}^{2} \xi\left(\widehat{u}_{0}, \nu\right) \oint \widetilde{a}_{1} \mathrm{~d} \widetilde{a}_{2}\right. \\
& \left.\quad-\nu_{1}^{2} \xi\left(\widehat{u}_{0}, \nu\right) \oint \widetilde{a}_{2} \mathrm{~d} \widetilde{a}_{1}+\nu_{1} \nu_{2} \oint \widetilde{a}_{2} \mathrm{~d} \widetilde{a}_{2}\right) .
\end{aligned}
$$

Now,

$$
\begin{aligned}
& \oint \widetilde{a}_{1} \mathrm{~d} \widetilde{a}_{1}=c \int_{\varphi=0}^{2 \pi} \cos \varphi \mathrm{d}(\cos \varphi)=0, \\
& \oint \widetilde{a}_{2} \mathrm{~d} \widetilde{a}_{2}=c \int_{\varphi=0}^{2 \pi} \sin \varphi \mathrm{d}(\sin \varphi)=0, \\
& \oint \widetilde{a}_{1} \mathrm{~d} \widetilde{a}_{2}=c \int_{\varphi=0}^{2 \pi} \cos \varphi \mathrm{d}(\sin \varphi)=c \int_{0}^{2 \pi} \cos ^{2} \varphi \mathrm{d} \varphi=\pi c, \\
& \oint \widetilde{a}_{2} \mathrm{~d} \widetilde{a}_{1}=c \int_{\varphi=0}^{2 \pi} \sin \varphi \mathrm{d}(\cos \varphi)=-c \int_{0}^{2 \pi} \sin ^{2} \varphi \mathrm{d} \varphi=-\pi c .
\end{aligned}
$$


Therefore,

$$
\begin{aligned}
& \widetilde{t}(\nu)=-\frac{16 \pi^{3}}{\|\nu\|^{4} \xi\left(\widehat{u}_{0}, \nu\right)^{2}}\left(\pi c \nu_{2}^{2} \xi\right.\left.\left(\widehat{u}_{0}, \nu\right)+\pi c \nu_{1}^{2} \xi\left(\widehat{u}_{0}, \nu\right)\right) \\
&=-\frac{16 \pi^{4} c}{\|\nu\|^{2} \xi\left(\widehat{u}_{0}, \nu\right)^{2}}=\frac{\widehat{u}(u, \nu) \widehat{u}(u,-\nu)}{\|\nu\|^{2} \xi\left(\widehat{u}_{0}, \nu\right)}
\end{aligned}
$$

There is also a slightly different method of calculating $\widetilde{t}(\nu)$ : with the reverse transformation (4.5.58), we can express $k_{1}$ and $k_{2}$ as functions of $\widetilde{z}$. Differentiating $k_{2}$ with respect to $\widetilde{z}$ lets us rewrite $k_{1} \mathrm{~d} k_{2}$ in the form $f(\widetilde{z}) \mathrm{d} \widetilde{z}$, where $f$ is a function holomorphic in $\mathbb{C} \backslash\{0\}$. We can then calculate the contour integral by applying Cauchy's residue theorem.

Pick a suitable $\delta>0$ in dependence of $u$. By theorem 4.5.42, the sequences $(\widehat{u}(u, \pm \nu))_{\nu \in \Gamma_{\delta}^{*}}$ are in $\ell^{\infty, 1}\left(\Gamma_{\delta}^{*}\right)$. Hence, by theorem 2.4.6. the product $(\widehat{u}(u, \nu) \widehat{u}(u,-\nu))_{\nu \in \Gamma_{\delta}^{*}}$ is in the quasi-Banach space $\ell^{\infty, 1 / 2}\left(\Gamma_{\delta}^{*}\right)$, which is continuously embedded in $\ell^{\infty, 1}\left(\Gamma_{\delta}^{*}\right)$ by propositions 2.4 .3 and 2.4.2. Also note that $(\widetilde{t}(\nu))_{\nu \in \Gamma_{\delta}^{*}}$ is even since $\xi\left(\widehat{u}_{0}, \nu\right)=\xi\left(\widehat{u}_{0},-\nu\right)$. Furthermore, $\xi\left(\widehat{u}_{0}, \nu\right)$ converges to one for $\|\nu\| \rightarrow \infty$. Hence, there are constants $C_{1}, C_{2}>0$ such that

$$
\frac{C_{1}}{n} \leq\left(\left(\frac{1}{\|\nu\|^{2} \xi\left(\widehat{u}_{0}, \nu\right)}\right)_{\nu \in \Gamma_{\delta}^{*}}\right)^{\star}(n) \leq \frac{C_{2}}{n}
$$

for all $n \in \mathbb{N}$. It therefore follows from the Hardy-Littlewood inequality (theorem 2.3.6 that $(\widetilde{t}(\nu))_{\nu \in \Gamma_{\delta}^{*}} \in \ell^{1}\left(\Gamma_{\delta}^{*}\right)$. With possibly different constants $C_{3}, C_{4}>$ 0 , we also have

$$
C_{3}|\widehat{u}(u, \nu) \widehat{u}(u,-\nu)| \leq\left|\frac{\widehat{u}(u, \nu) \widehat{u}(u,-\nu)\left(1+4 \pi^{2}\|\nu\|^{2}\right)}{\|\nu\|^{2} \xi\left(\widehat{u}_{0}, \nu\right)}\right| \leq C_{4}|\widehat{u}(u, \nu) \widehat{u}(u,-\nu)|
$$

for all $\nu \in \Gamma_{\delta}^{*}$. But $1+4 \pi^{2}\|\nu\|^{2}$ is the Fourier transform of the Bessel kernel of second order (cf. theorem 2.2.13). Hence $(\widetilde{t}(\nu))_{\nu \in \Gamma_{\delta}^{*}}$ lies in the secondorder Bessel space of $\ell^{\infty, 1 / 2}\left(\Gamma_{\delta}^{*}\right)_{e}$, which we denote by $\mathcal{B}^{2} \ell^{\infty, 1 / 2}\left(\Gamma_{\delta}^{*}\right)_{e}$. Now, take $\left(a_{\nu}\right)_{\nu \in \Gamma_{\delta}^{*}} \in \ell^{\infty, 1 / 2}\left(\Gamma_{\delta}^{*}\right)_{e}$ and set $b_{\nu}:=\sqrt{a_{\nu}}$ (choose one square root for each distinct value of $\left.\left(a_{\nu}\right)_{\nu \in \Gamma_{\delta}^{*}}\right)$ for all $\nu \in \Gamma_{\delta}^{*}$. Then $a_{\nu}=b_{\nu} b_{-\nu}$ due to evenness. Now, since for all sequences the equality $\sqrt{a^{\star}}=\sqrt{a^{\star}}$ holds, we have

$$
\sum_{n=1}^{\infty} \frac{b^{\star}(n)}{n}=\sum_{n=1}^{\infty} \frac{\sqrt{a^{\star}(n)}}{n}<\infty
$$

Hence, the mapping from $\ell^{\infty, 1}\left(\Gamma_{\delta}^{*}\right)$ to $\ell^{\infty, 1 / 2}\left(\Gamma_{\delta}^{*}\right)_{e}$ is onto.

In the preceding proof, we mentioned an alternate method of calculating the model moduli, using Cauchy's residue theorem on a function holomorphic in a punctured neighbourhood of zero. This method extends to the case where $\widehat{u}(u, \nu)=0$ or $\widehat{u}(u,-\nu)=0$ and yields zero, just as defined explicitly in definition 4.5.55 The geometric interpretation of this fact is that the handle vanishes. Hence, we have arrived at a geometrically sensible definition of an unsplit double point.

Definition 4.5.61. For $u \in \mathcal{F} \ell^{\infty, 1}\left(\Gamma^{*}\right)$ we call the double point at $k_{\nu}^{ \pm}\left(\widehat{u}_{0}\right)$, $\nu \in \Gamma_{\delta}^{*}$ with sufficiently small $\delta>0$, unsplit if $\widehat{u}(u, \nu)=0$ or $\widehat{(u,-\nu)}=0$. 
This definition contrasts with our definition of finite-type potentials 4.5.32. There, we effectively required the matrix 4.5 .20$)$ to become zero at $k_{ \pm}$, while here we are satisfied with the matrix being nilpotent. In both cases the eigenspace of zero is two-dimensional, but for a nilpotent matrix, there need not exists two linearly independent eigenvectors.

Let us now generalise theorem 4.5 .56 to the actual Fermi curve.

Definition 4.5.62. In analogy with definition 4.5.55 we define the modulus of the Fermi curve with respect to $\nu \in \Gamma_{\delta}^{*}$ for a suitable $\delta>0$ as

$$
t(\nu):= \begin{cases}0 & \text { if the corresponding double point remains unsplit } \\ 16 \pi^{3} \oint k_{1} \mathrm{~d} k_{2} & \text { otherwise. }\end{cases}
$$

This is virtually the same definition as definition 4.5.55 except that the relation between $k_{1}$ and $k_{2}$ is different. This difference can be regarded as a perturbation of the model Fermi curve yielding the actual Fermi curve (at a single handle). In particular, the results of theorem 4.5.56 carry over.

Theorem 4.5.63. For all $u \in \mathcal{F} \ell^{\infty, 1}\left(\Gamma^{*}\right)$ there is a $\delta>0$ such that the corresponding moduli $t(\nu)$ are well-defined for all $\nu \in \Gamma_{\delta}^{*}$, with $(t(\nu))_{\nu \in \Gamma_{\delta}^{*}}$ belonging to the second-order Bessel potential space of the quasi-Banach space $\ell^{\infty, 1 / 2}\left(\Gamma_{\delta}^{*}\right)_{e}$, and the mapping $u \mapsto t$ is locally onto.

Proof. Let us have a look at 4.5.20 near $k=k_{ \pm \nu}$. Set

$K_{1}\left(k, \widehat{u}_{0}\right):=-4 \pi^{2}\left(\left(k+k_{\nu}^{ \pm}\left(\widehat{u}_{0}\right)\right)^{2}+\widehat{u}_{0}\right), \quad K_{2}\left(k, \widehat{u}_{0}\right):=-4 \pi^{2}\left(\left(k+k_{\nu}^{\mp}\left(\widehat{u}_{0}\right)\right)^{2}+\widehat{u}_{0}\right)$,

and denote the four entries of $\mathcal{A}_{ \pm \nu}\left(k+k_{\nu}^{ \pm}\left(\widehat{u}_{0}\right), u\right)$ by $A_{11}(k, u), A_{12}(k, u)$, $A_{21}(k, u)$ and $A_{22}(k, u)$, respectively (in this notation, we have suppressed the dependence on $\nu$ ). Then we can rewrite (4.5.20) as

$$
\begin{aligned}
& \left(\begin{array}{cc}
K_{1}\left(k, \widehat{u}_{0}\right)+A_{11}(k, u) & A_{12}(k, u) \\
A_{21}(k, u) & K_{2}\left(k, \widehat{u}_{0}\right)+A_{22}(k, u)
\end{array}\right) \\
= & \left(\begin{array}{cc}
K_{1}\left(k, \widehat{u}_{0}\right)-K_{1}\left(k_{ \pm \nu}, \widehat{u}_{0}\right) & A_{12}\left(k_{ \pm \nu}, u\right) \\
+K_{1}\left(k_{ \pm \nu}, \widehat{u}_{0}\right)+A_{11}(k, u) & +A_{12}(k, u)-A_{12}\left(k_{ \pm \nu}, u\right) \\
A_{21}\left(k_{ \pm \nu}, u\right) & K_{2}\left(k, \widehat{u}_{0}\right)-K_{2}\left(k_{ \pm \nu}, \widehat{u}_{0}\right) \\
+A_{21}(k, u)-A_{21}\left(k_{ \pm \nu}, u\right) & +K_{2}\left(k_{ \pm \nu}, \widehat{u}_{0}\right)+A_{22}(k, u)
\end{array}\right)
\end{aligned}
$$

By definition 4.5.31, $K_{1}\left(k_{ \pm \nu}, \widehat{u}_{0}\right)=-A_{11}\left(k_{ \pm \nu}, u\right), A_{12}\left(k_{ \pm \nu}, u\right)=\widehat{u}(u, \pm \nu)$, $A_{21}\left(k_{ \pm \nu}, u\right)=\widehat{u}(u, \mp \nu)$, and $K_{2}\left(k_{ \pm \nu}, \widehat{u}_{0}\right)=-A_{22}\left(k_{ \pm \nu}, u\right)$, so

$$
=\left(\begin{array}{cc}
K_{1}\left(k, \widehat{u}_{0}\right)-K_{1}\left(k_{ \pm \nu}, \widehat{u}_{0}\right) & \widehat{u}(u, \pm \nu) \\
+A_{11}(k, u)-A_{11}\left(k_{ \pm \nu}, u\right) & +A_{12}(k, u)-A_{12}\left(k_{ \pm \nu}, u\right) \\
\widehat{u}(u, \mp \nu) & K_{2}\left(k, \widehat{u}_{0}\right)-K_{2}\left(k_{ \pm \nu}, \widehat{u}_{0}\right) \\
+A_{21}(k, u)-A_{21}\left(k_{ \pm \nu}, u\right) & +A_{22}(k, u)-A_{22}\left(k_{ \pm \nu}, u\right)
\end{array}\right) .
$$


Taking the determinant of this matrix and setting it to zero yields

$$
\begin{aligned}
& \left(\left(k_{1}-k_{ \pm \nu, 1}\right)\left(-\nu_{1}+i \nu_{2} \xi\left(\widehat{u}_{0}, \nu\right)+k_{1}+k_{ \pm \nu, 1}\right)\right. \\
& \quad+\left(k_{2}-k_{ \pm \nu, 2}\right)\left(-i \nu_{1} \xi\left(\widehat{u}_{0}, \nu\right)-\nu_{2}+k_{2}+k_{ \pm \nu, 2}\right) \\
& \left.\quad+A_{11}(k, u)-A_{11}\left(k_{ \pm \nu}, u\right)\right) \\
& \cdot\left(\left(k_{1}-k_{ \pm \nu, 1}\right)\left(\nu_{1}+i \nu_{2} \xi\left(\widehat{u}_{0}, \nu\right)+k_{1}+k_{ \pm \nu, 1}\right)\right. \\
& \quad+\left(k_{2}-k_{ \pm \nu, 2}\right)\left(-i \nu_{1} \xi\left(\widehat{u}_{0}, \nu\right)+\nu_{2}+k_{2}+k_{ \pm \nu, 2}\right) \\
& \left.\quad+A_{22}(k, u)-A_{22}\left(k_{ \pm \nu}, u\right)\right) \\
& =\frac{\left(\widehat{u}(u, \pm \nu)+A_{12}(k, u)-A_{12}\left(k_{ \pm \nu}, u\right)\right)\left(\widehat{u}(u, \mp \nu)+A_{21}(k, u)-A_{21}\left(k_{ \pm \nu}, u\right)\right)}{-16 \pi^{4}} .
\end{aligned}
$$

We can absorb all $k$-dependent parts of the right hand side of this equation into the left hand side by writing

$$
\begin{gathered}
\left(\left(k_{1}-k_{ \pm \nu, 1}\right)\left(-\nu_{1}+i \nu_{2} \xi\left(\widehat{u}_{0}, \nu\right)+k_{1}+k_{ \pm \nu, 1}\right)\right. \\
\quad+\left(k_{2}-k_{ \pm \nu, 2}\right)\left(-i \nu_{1} \xi\left(\widehat{u}_{0}, \nu\right)-\nu_{2}+k_{2}+k_{ \pm \nu, 2}\right) \\
\left.+A_{11}(k, u)-A_{11}\left(k_{ \pm \nu}, u\right)\right) \\
\cdot\left(\left(k_{1}-k_{ \pm \nu, 1}\right)\left(\nu_{1}+i \nu_{2} \xi\left(\widehat{u}_{0}, \nu\right)+k_{1}+k_{ \pm \nu, 1}\right)\right. \\
\quad+\left(k_{2}-k_{ \pm \nu, 2}\right)\left(-i \nu_{1} \xi\left(\widehat{u}_{0}, \nu\right)+\nu_{2}+k_{2}+k_{ \pm \nu, 2}\right) \\
\left.\quad+A_{22}(k, u)-A_{22}\left(k_{ \pm \nu}, u\right)+P\left(k, k_{ \pm \nu}, u, \nu\right)\right) \\
=\frac{\widehat{u}(u, \nu) \widehat{u}(u,-\nu)}{-16 \pi^{4}}
\end{gathered}
$$

where

$$
\begin{aligned}
P\left(k, k_{ \pm \nu}, u, \nu\right):=(\widehat{u}(u, & \pm \nu)\left(A_{21}(k, u)-A_{21}\left(k_{ \pm \nu}, u\right)\right) \\
& +\widehat{u}(u, \mp \nu)\left(A_{12}(k, u)-A_{12}\left(k_{ \pm \nu}, u\right)\right) \\
+ & \left.\left(A_{12}(k, u)-A_{12}\left(k_{ \pm \nu}, u\right)\right)\left(A_{21}(k, u)-A_{21}\left(k_{ \pm \nu}, u\right)\right)\right) \\
/( & -16 \pi^{4}\left(\left(k_{1}-k_{ \pm \nu, 1}\right)\left(-\nu_{1}+i \nu_{2} \xi\left(\widehat{u}_{0}, \nu\right)+k_{1}+k_{ \pm \nu, 1}\right)\right. \\
& +\left(k_{2}-k_{ \pm \nu, 2}\right)\left(-i \nu_{1} \xi\left(\widehat{u}_{0}, \nu\right)-\nu_{2}+k_{2}+k_{ \pm \nu, 2}\right) \\
+ & \left.\left.A_{11}(k, u)-A_{11}\left(k_{ \pm \nu}, u\right)\right)\right) .
\end{aligned}
$$

This expression has a single singularity at $k=k_{ \pm \nu}$ and is otherwise holomorphic for all $\nu \in \Gamma_{\delta}^{*}$ for sufficiently small $\delta>0$ because $V$ is bounded. In fact, this singularity is removeable because both numerator and denominator have a zero at $k=k_{ \pm \nu}$, and the zero in the denominator is only of first order. The right hand side of 4.5.64 now equals $c$ from the proof of theorem 4.5.56. Let us now 
generalise some quantities from this theorem. We set

$$
\begin{aligned}
a_{1}:= & i\left(k_{1}-k_{ \pm \nu, 1}\right)\left(\nu_{2} \xi\left(\widehat{u}_{0}, \nu\right)+k_{1}+k_{ \pm \nu, 1}\right) \\
& -i\left(k_{2}-k_{ \pm \nu, 2}\right)\left(\nu_{1} \xi\left(\widehat{u}_{0}, \nu\right)+k_{2}+k_{ \pm \nu, 2}\right) \\
& +\frac{1}{2}\left(A_{11}(k, u)-A_{11}\left(k_{ \pm \nu}, u\right)\right)+\frac{1}{2}\left(A_{22}(k, u)-A_{22}\left(k_{ \pm \nu}, u\right)\right) \\
& +\frac{1}{2} P\left(k, k_{ \pm \nu}, u, \nu\right), \\
a_{2}:= & i\left(k_{1}-k_{ \pm \nu, 1}\right) \nu_{1}+i\left(k_{2}-k_{ \pm \nu, 2}\right) \nu_{2} \\
& -\frac{i}{2}\left(A_{11}(k, u)-A_{11}\left(k_{ \pm \nu}, u\right)\right)+\frac{i}{2}\left(A_{22}(k, u)-A_{22}\left(k_{ \pm \nu}, u\right)\right) \\
& +\frac{i}{2} P\left(k, k_{ \pm \nu}, u, \nu\right) .
\end{aligned}
$$

This is a generalisation of the quantities $\widetilde{a}_{1}, \widetilde{a}_{2}$, and again, equation 4.5.64 simplifies to $\left(a_{1}+i a_{2}\right)\left(a_{1}-i a_{2}\right)=c$. Setting $z:=a_{1}+i a_{2}$, we again get the equations

$$
a_{1}:=\frac{1}{2}\left(z+\frac{c}{z}\right), \quad a_{2}:=\frac{1}{2 i}\left(z-\frac{c}{z}\right) .
$$

We would like to parameterise our integration contour similar to $z=c \exp (i \varphi)$, as we did earlier. However, it must be noted that the quantities $a_{1}, a_{2}$ and $z$ are not defined in a domain as large as the domain of $\widetilde{a}_{1}, \widetilde{a}_{2}$ and $\widetilde{z}$, respectively, for the matrices $A_{11}, A_{12}, A_{21}$ and $A_{22}$ are not defined for all $k$. In fact, with respect to definition 4.5.31 and lemma 4.5.53, we would like to keep $k$ in a neighbourhood of $k_{ \pm \nu}$. In the case of our Fermi curve model, we can calculate $k-k_{ \pm \nu}$ explicitly using (4.5.58), to wit

$$
\begin{aligned}
& k_{1}-k_{ \pm \nu, 1}=\frac{\sqrt{c}}{i\|\nu\|^{2} \xi\left(\widehat{u}_{0}, \nu\right)}\left(\nu_{2} \cos \varphi+\nu_{1} \xi\left(\widehat{u}_{0}, \nu\right) \sin \varphi\right), \\
& k_{2}-k_{ \pm \nu, 2}=\frac{\sqrt{c}}{i\|\nu\|^{2} \xi\left(\widehat{u}_{0}, \nu\right)}\left(-\nu_{1} \cos \varphi+\nu_{2} \xi\left(\widehat{u}_{0}, \nu\right) \sin \varphi\right) .
\end{aligned}
$$

Since $\sqrt{c}$ itself is in $\ell^{\infty, 1}\left(\Gamma_{\delta}^{*}\right)_{e}$ by theorem 4.5.42, we see that $\left\|k-k_{ \pm \nu}\right\|$ is in a space we denote by $\ell^{\infty, 1}\left(\Gamma_{\delta}^{*}\right) /\|\nu\|$, that is, $\|\nu\|\left\|k-k_{ \pm \nu}\right\|$ is in $\ell^{\infty, 1}\left(\Gamma_{\delta}^{*}\right)$ (in particular, $\left\|k-k_{ \pm \nu}\right\|$ is in $\ell^{2,1}\left(\Gamma_{\delta}^{*}\right)$ ). Note that $\left\|k-k_{ \pm \nu}\right\| \leq|\sqrt{c}|$ for all sufficiently small $\delta>0$, so with a small perturbation of the model Fermi curve, the contour will still encompass the whole handle. Hence, the integral remains well-defined.

In the case of the actual Fermi curve, we don't have an explicit inversion formula. However, by lemma 4.5.21 the derivative of $\left(a_{1}, a_{2}\right)$ with respect to $k-$ $k_{ \pm \nu}$ is regular in a neighbourhood of $k_{ \pm \nu}$. Hence, there exists an inverse $a^{-1}$ such that $a^{-1}\left(a_{1}, a_{2}\right)=k-k_{ \pm \nu}$ in a neighbourhood of $k_{ \pm \nu}$. In the model case, $a^{-1}$ is simply given by (4.5.58). Thus, in order to estimate the perturbation of $k$ when passing from the model Fermi curve to the actual Fermi curve, it is sufficient to estimate the variation of $a^{-1}$ and use the mean value theorem, because the difference $\left(a_{1}, a_{2}\right)-\left(\widetilde{a}_{1}, \widetilde{a}_{2}\right)$ is bounded by some constant because $V$ is bounded. But now,

$$
\left(a^{-1}\right)^{\prime}(\varphi)=\left(\frac{\partial}{\partial k}\left(a_{1}, a_{2}\right)\right)^{-1} \frac{\partial}{\partial \varphi}\left(a_{1}, a_{2}\right) .
$$


We can work out the right hand side by explicit calculation. Since sine and cosine are bounded, the $\varphi$-derivative of $a_{1}$ and $a_{2}$ is in $\ell^{\infty, 1}\left(\Gamma_{\delta}^{*}\right)_{e}$ uniformly. The $k$-derivative is more complicated. First, note that

$$
\begin{aligned}
\frac{\partial}{\partial k_{1}} P\left(k, k_{ \pm \nu}, u, \nu\right)=( & \left(\widehat{u}(u, \pm \nu)+A_{12}(k, u)-A_{12}\left(k_{ \pm \nu}, u\right)\right) \frac{\partial}{\partial k_{1}} A_{21}(k, u) \\
+ & \left(\widehat{u}(u, \mp \nu)+A_{21}(k, u)\right. \\
& \left.\left.-A_{21}\left(k_{ \pm \nu}, u\right)\right) \frac{\partial}{\partial k_{1}} A_{12}(k, u)\right) \\
/ & \left(-16 \pi^{4}\left(\left(k_{1}-k_{ \pm \nu, 1}\right)\left(-\nu_{1}+i \nu_{2} \xi\left(\widehat{u}_{0}, \nu\right)+k_{1}+k_{ \pm \nu, 1}\right)\right.\right. \\
+ & \left(k_{2}-k_{ \pm \nu, 2}\right)\left(-i \nu_{1} \xi\left(\widehat{u}_{0}, \nu\right)-\nu_{2}+k_{2}+k_{ \pm \nu, 2}\right) \\
& \left.\left.+A_{11}(k, u)-A_{11}\left(k_{ \pm \nu}, u\right)\right)\right) \\
+( & \left(\widehat{u}(u, \pm \nu)\left(A_{21}(k, u)-A_{21}\left(k_{ \pm \nu}, u\right)\right)\right. \\
+ & \widehat{u}(u, \mp \nu)\left(A_{12}(k, u)-A_{12}\left(k_{ \pm \nu}, u\right)\right) \\
+ & \left(A_{12}(k, u)-A_{12}\left(k_{ \pm \nu}, u\right)\right) \\
& \left.\cdot\left(A_{21}(k, u)-A_{21}\left(k_{ \pm \nu}, u\right)\right)\right) \\
& \left.\cdot\left(-\nu_{1}+i \nu_{2} \xi\left(\widehat{u}_{0}, \nu\right)+\frac{\partial}{\partial k_{1}} A_{11}(k, u)\right)\right) \\
/ & \left(1 6 \pi ^ { 4 } \left(\left(k_{1}-k_{ \pm \nu, 1}\right)\left(-\nu_{1}+i \nu_{2} \xi\left(\widehat{u}_{0}, \nu\right)+k_{1}+k_{ \pm \nu, 1}\right)\right.\right. \\
& +\left(k_{2}-k_{ \pm \nu, 2}\right)\left(-i \nu_{1} \xi\left(\widehat{u}_{0}, \nu\right)-\nu_{2}+k_{2}+k_{ \pm \nu, 2}\right) \\
& \left.\left.+A_{11}(k, u)-A_{11}\left(k_{ \pm \nu}, u\right)\right)^{2}\right) .
\end{aligned}
$$

The expression for $\left(\partial / \partial k_{2}\right) P\left(k, k_{ \pm \nu}, u, \nu\right)$ is similar. Therefore, it follows from corollary 4.5 .52 and the mean value theorem that these derivatives are in the same space as $\left\|k-k_{ \pm \nu}\right\|$ with respect to $\nu$ as in the model case. By the same corollary, the derivative of $\left(a_{1}, a_{2}\right)$ with respect to $k$ is asymptotically equal to the linear operator given by

$$
i\left(\begin{array}{cc}
\nu_{2} \xi\left(\widehat{u}_{0}, \nu\right)+2 k_{1} & -\nu_{1} \xi\left(\widehat{u}_{0}, \nu\right)-2 k_{2} \\
\nu_{1} & \nu_{2}
\end{array}\right)
$$

the inverse of which is given by

$$
\frac{i}{\|\nu\|^{2} \xi\left(\widehat{u}_{0}, \nu\right)+2\left(\nu_{1} k_{2}+\nu_{2} k_{1}\right)}\left(\begin{array}{rr}
-\nu_{2} & -\nu_{1} \xi\left(\widehat{u}_{0}, \nu\right)-2 k_{2} \\
\nu_{1} & -\nu_{2} \xi\left(\widehat{u}_{0}, \nu\right)-2 k_{1}
\end{array}\right) .
$$

This expression is of order $O(1 /\|\nu\|)$, corresponding to the space $\ell^{2, \infty}\left(\Gamma_{\delta}^{*}\right)$, which encompasses $\ell^{\infty, 1}\left(\Gamma_{\delta}^{*}\right) /\|\nu\|$. Hence, taking both terms together, the $\varphi$ derivative of $a^{-1}$ can be expressed by multiplying the components with functions in $\ell^{\infty, 1}\left(\Gamma_{\delta}^{*}\right) /\|\nu\|$. It follows that for the actual Fermi curve, $\left\|k-k_{ \pm \nu}\right\|$ is in the same space as for the model Fermi curve. This holds uniformly for all $\varphi$. For the same reasons, in the form

$$
\left(k_{1}-k_{ \pm \nu, 1}\right) \mathrm{d}\left(k_{2}-k_{ \pm \nu, 2}\right)=\left(k_{1}-k_{ \pm \nu, 1}\right) k_{2}^{\prime}\left(a_{1}, a_{2}\right)\left((\partial / \partial \varphi)\left(a_{1}, a_{2}\right)\right) \mathrm{d} \varphi,
$$


the product is, with respect to $\nu$, of the form

$$
\left(\ell^{\infty, 1}\left(\Gamma_{\delta}^{*}\right) /\|\nu\|\right) \cdot O(1 /\|\nu\|) \cdot \ell^{\infty, 1}\left(\Gamma_{\delta}^{*}\right),
$$

giving $\mathcal{B}^{2} \ell^{\infty, 1 / 2}\left(\Gamma_{\delta}^{*}\right)$, uniformly in $\varphi$. Since the contour is compact, the same holds for $(t(\nu))_{\nu \in \Gamma_{\delta}^{*}}$. Furthermore, due to proposition 4.5.8 $(t(\nu))_{\nu \in \Gamma_{\delta}^{*}}$ is even. By theorem 4.5.56 $u \mapsto t$ is locally onto as a regular variation of $u \mapsto \widetilde{t}$.

Corollary 4.5.65. The moduli space of Fermi curves for $\mathcal{F} \ell^{\infty, 1}\left(\Gamma^{*}\right)$-potentials can be identified with a $\mathcal{B}^{2} \ell_{e}^{\infty, 1 / 2}$ quasi-Banach manifold. 


\section{Chapter 5}

\section{Summary and outlook}

\subsection{Works with different focus}

To a large part, the focus of this work has been on functional analysis. Before we summarise, let us name just two examples of works with a different focus.

In [NV97] (see also the references therein), two-dimensional Schrödinger operators with and without magnetic field (the latter are dubbed purely potential) are treated within the context of the theory of integrable systems and solitons. In this context, a special class of exactly solvable Schrödinger operators is defined. These operators correspond to Fermi curves with finite genus. For the purely potential operators, the solution of the inverse problem yields a Baker-Akhiezer eigenfunction of a Schrödinger operator from a Riemann surface of finite genus adorned with a divisor and a pair of "infinitely distant" points, that is, an eigenfunction for each $k$ from the surface. The resultant Baker-Akhiezer function is unique up to multiplication with a $k$-independent function. This result is somewhat of a counterpoint to our own result because we are not at all interested in the finite-genus situation (in fact, we hide a finite number of handles away in the "compact part" of the Fermi curve) but rather in the asymptotics of a Fermi curve with infinite genus.

In [FKT03], marked Riemann surfaces satisfying certain geometric hypotheses are introduced. The markings and hypotheses correspond to a construction of a Riemann surface of infinite genus consisting of a finite-genus compact submanifold, a finite number of regular pieces, which are essentially biholomorphic to $\mathbb{C}$, except that they are glued to the compact submanifold and connected with an infinite number of separate handles, such that the size of the handles abates with distance from the origin in a certain way. This setting is compatible with our results from section 4.3 . However, only later are specific examples treated, among them two-dimensional periodic Schrödinger equations. The potentials are taken from various subspaces of $L^{2}(F)$. The Fermi curves are treated in a setting which is a generalisation to infinite genera of the classical theory of compact (and hence finite-genus) Riemann surfaces. The genericity of this approach is an asset, as it can also be applied to heat curves (the analogon of the Fermi curves for the heat equation) and in the context of the Kadomtsev-Petviashvili (KP) equation. Indeed, for these Riemann surfaces fulfilling the geometric hypotheses, the potential of the corresponding KP equation (and thus the potential 
of the one-dimensional Schrödinger equation) is reconstructed through the surface's theta series (at least for smooth potentials). A similar procedure should also be possible for our Schrödinger equation.

Let us now summarise and discuss our new results.

\subsection{Compact resolvent}

The spectral theory of the Schrödinger operator is a classic topic, including the definition of the resolvent as a meromorphic, operator-valued function yielding compact operators on $L^{2}(F)$, as we did in chapter 3 . Our goal was to repeat this feat with Schrödinger potentials as general as possible, at least for $d=2$. For $d>$ 2 , we didn't go out of our way to find the largest sensible space, because in this case, the standard Sobolev and Banach space localisation theory works very well and also shows that, at least on the $L^{p}$-scale, $L^{d / 2}(F)$ is the limit (for $d \geq 5$, this has been known for some time, see e.g. [RS78, theorem XIII.99]). It should be possible to do a little better. With the help of the Hardy-Littlewood-Sobolev theorem [BS88, Theorem 4.4.18] and Hölder's inequality, theorem 2.4.6, the key lemma 3.3.4 should be generalisable to potentials from $L^{d / 2, r}(F)$, with $r<\infty$ (note that since $d>2$, this space becomes a Banach space by passing from the decreasing rearrangement to the maximal function in the definition of the Lorentz-Zygmund spaces). It is doubtful whether generalisation to $L^{d / 2, \infty}(F)$ is possible for large potentials, because, while Hölder's inequality still holds, theorem 2.8.2 does not apply in this case. If it is not possible, then there is no largest space of potentials in terms of Lorentz spaces. This would be quite unsatisfying. In any case, Sobolev theory seems to be the simplest and most natural way to treat the $d>2$ case, as it serves exactly the function it was created for.

For $d=2$, the situation is quite different. Here, $L^{1}(F)$ is too large a space of potentials, while all $L^{p}(F), p>1$, would serve. Hence, an optimal Lebesgue space of potentials does not exist. In subsection 2.7.1. we already explained why the Lorentz-Zygmund space $L^{1,1 ; 1}(F)$ is the optimal subspace of $L^{1}(F)$ to use for the domain of the resolvent. For the potentials, it turned out that the optimal subspace of $L^{1}(F)$ is also $L^{1,1 ; 1}(F)$ (recall that for $d>2$, these two spaces were different), by a result of Stein. The step from $L^{1,1 ; 1}(F)$ to the Fourier space $\mathcal{F} \ell^{\infty, 1}\left(\Gamma^{*}\right)$ was inspired by chapter 4 , where it is much more natural to view the potential as a Fourier series than as an ordinary function. The execution of the spectral theory programme for the resolvent of the Schrödinger operator for the space of potentials $\mathcal{F} \ell^{\infty, 1}\left(\Gamma^{*}\right)$ as done in chapter 3 is a new result. With respect to the generality of the potential, it improves significantly on earlier work, where potentials are usually taken from $L^{2}(F)$ or even less general spaces (we did, though, publish some preliminary results in KS09). By theorem 2.4.12. $\mathcal{F} \ell^{\infty, 1}\left(\Gamma^{*}\right)$ not only contains the optimal $L^{1}(F)$-subspace $L^{1,1 ; 1}(F)$, but also some nonintegrable distributions. Calculations with $\mathcal{F} \ell^{\infty, 1}\left(\Gamma^{*}\right)$ are well-suited for our purposes, as can be seen in the proof of the key lemma 3.3 .6 as well as in the proof of proposition 4.5.35, where we make use of the fact that the rearrangements of $\ell^{1}\left(\Gamma^{*}\right)$ are precisely the Cauchy condensations of the rearrangements of $\ell^{\infty, 1}\left(\Gamma^{*}\right)$.

The question remains whether there are spaces different from $\mathcal{F} \ell^{\infty, 1}\left(\Gamma^{*}\right)$ which work better or at least equally well. For example, theorem 2.4 .12 yields 
a whole family of possible target spaces for the resolvent domain, namely the spaces $\mathcal{F} \ell^{\infty, b ; \beta}\left(\Gamma^{*}\right)$, with $b^{-1}+\beta=1$ and $1 \leq b \leq \infty$. However, the Fourier transform of the free resolvent is a multiplier in $\ell^{1, \infty}\left(\Gamma^{*}\right)$, and the generalisation of Hölder's inequality 2.4.6 does not extend to the case $b>1$ in the instance $\ell^{1, \infty}\left(\Gamma^{*}\right) \cdot \ell^{\infty, b ; \beta}\left(\Gamma^{*}\right)$. This would lead to trouble in the proof of lemma 3.3.6. Furthermore, the multiplier does not belong to the space $\ell^{1, a ; \alpha}\left(\Gamma^{*}\right)$ with $a^{-1}+\alpha=0, a<\infty$ either. As a quick calculation shows, attempting to place the multiplier in one of these spaces always leads to a divergence of type

$$
\sum \frac{1}{n \log n} .
$$

Hence, $\mathcal{F} \ell^{\infty, 1}\left(\Gamma^{*}\right)$ is optimal in terms of Lorentz-Zygmund spaces.

On the other hand, we see from the proof of theorem 3.3 .24 that, abstractly, the most general set of potentials is the set of those $u$ for which the operator $\left(\lambda_{0}+\right.$ $\left.\Delta_{k}-u\right)^{-1}$ is a compact endomorphism on $L^{2}(F)$, for $k \in V \subseteq \mathbb{C}^{2}$ bounded and some $\lambda_{0} \in \mathbb{C}$. Since this does not directly yield a Banach space of potentials, we go some steps further back, to the two factorisations (3.3.18). Since the free resolvent must be defined whenever the general resolvent is, we see that a necessary condition on the potentials $u$ is that the operators $u\left(\lambda+\Delta_{0}\right)^{-1}$ and $\left(\lambda+\Delta_{0}\right)^{-1} u$ are bounded endomorphisms on suitable spaces $E_{1}$ and $E_{2}$, respectively. Since $u$ appears linearly in these operators, it may seem possible to define norms for $u$ by

$$
\begin{gathered}
\|u\|:=\sup _{\substack{f \in E_{1} \\
\|f\|_{E_{1}} \leq 1}}\left\|u\left(\lambda+\Delta_{0}\right)^{-1} f\right\|_{E_{1}} \quad \text { and } \\
\|u\|:=\sup _{\substack{f \in E_{2} \\
\|f\|_{E_{2}} \leq 1}}\left\|\left(\lambda+\Delta_{0}\right)^{-1} u f\right\|_{E_{2}} .
\end{gathered}
$$

This ansatz is not very fruitful, though. It is a simple generalisation of the concept of associate spaces (see [BS88, 1.2, 2.4]). In the context of Banach function spaces, well-definedness and completeness of the associate space is automatic, and rearrangement-invariance is hereditary. With our ansatz, we have to make sure that the free resolvent maps $E_{1}$ into $E_{2}$, and that the multiplication with $u$ maps $E_{2}$ into $E_{1}$. In order to obtain a Banach space, these mappings must also be bounded. Apart from that, and $L^{2}(F) \subseteq E_{1}$ and $E_{2} \subseteq L^{2}(F)$, it is also not clear which Banach spaces we should use for $E_{1}$ and $E_{2}$. Simple considerations, such as [BS88, Lemma 3.1.9], show that $E_{1}$ and $E_{2}$ should be chosen as large as possible in order to not miss out on potentials. On the other hand, $E_{1}$ and $E_{2}$ do not have any bearing on the spectrum of the resolvent whatsoever, provided the resolvent is compact on $L^{2}(F)$. Attempting to use $E_{2}=L^{2}(F)$, anyway, does not appear to yield new results.

Let us note, however, that the theory of associate spaces of rearrangementinvariant function spaces gives us a slight hint that there may yet be more potentials to make the resolvent compact. The space $\ell^{\infty, 1}\left(\Gamma^{*}\right)$, being a rearrangementinvariant function space, has an associate space $\left(\ell^{\infty, 1}\left(\Gamma^{*}\right)\right)^{\prime}$, which is also a rearrangement-invariant Banach function space. This is the largest space, such that the Hölder inequality

$$
\|f g\|_{1} \leq\|f\|_{\ell \infty, 1\left(\Gamma^{*}\right)}\|g\|_{\left(\ell^{\infty, 1}\left(\Gamma^{*}\right)\right)^{\prime}}
$$


holds. The Hölder inequality for Lorentz-Zygmund spaces shows that we have the bounded embedding $\ell^{1, \infty}\left(\Gamma^{*}\right) \subseteq\left(\ell^{\infty, 1}\left(\Gamma^{*}\right)\right)^{\prime}$. Now, $\ell^{1, \infty}\left(\Gamma^{*}\right)$ is only a quasiBanach function space, but the generalisation of the concept of the associate space to quasi-Banach function spaces is straightforward. In particular, BS88, Proposition 1.2.10] continues to hold. Choose $E_{2}=\mathcal{F} \ell^{1}\left(\Gamma^{*}\right)$, then we may take $u \in \mathcal{F}\left(\ell^{1, \infty}\left(\Gamma^{*}\right)\right)^{\prime}$. Since $\ell^{\infty, 1}\left(\Gamma^{*}\right) \subseteq\left(\ell^{1, \infty}\left(\Gamma^{*}\right)\right)^{\prime}$ boundedly, we may thus have gained a greater space. Of course, $\left(\ell^{1, \infty}\left(\Gamma^{*}\right)\right)^{\prime}$ is a priori only a quasiBanach space. Furthermore, we have not investigated whether a quasi-norm localisation is possible, so that we can apply the Neumann theorem (the achieved bound would have to be smaller than the inverse of the constant on the triangle inequality in this case).

Another way of looking at this problem is to use the hindsight gained through the results of subsection 4.5.3. Locally, the moduli reside in a space slightly smaller than the smallest rearrangement-invariant Banach space $\ell^{1}\left(\Gamma_{\delta}^{*}\right)$. From what space would the perturbed Fourier coefficients $\widehat{u}(u, \nu), \nu \in \Gamma_{\delta}^{*}$ have to come, so that the moduli locally reside precisely in (the even part of $) \ell^{1}\left(\Gamma_{\delta}^{*}\right)$ ? A simple calculation shows that this would have to be something like the space

$$
\sqrt{\|\nu\|^{2} \cdot \ell^{1}\left(\Gamma^{*}\right)},
$$

(for simplicity, we are leaving out the $\delta$ ) which is simply the space of $\ell^{2}\left(\Gamma_{\delta}^{*}\right)$ functions multiplied with $\|\nu\|$. Due to Plancherel's theorem, this space is the the Fourier space of the negative Sobolev space $W^{-1,2}(F)$, that is, the dual space of $W^{1,2}(F)$ [Lax55, section 2]. Unfortunately, this space is definitely too big for the Fourier transforms of the potentials. The action of the Bessel kernel of second order is the same as twice the action of the Bessel kernel of first order. The Bessel kernel of first order maps $\mathcal{F} \ell^{\infty, 2}\left(\Gamma^{*}\right)$ into $\mathcal{F} \ell^{2,2}\left(\Gamma^{*}\right)=L^{2}(F)$ by the Hardy-Littlewood inequality, and also $L^{2}(F)$ into the Sobolev space $W^{1,2}(F)$ by the (now safe) theorem 2.2.14. Therefore, $\mathcal{F} \ell^{\infty, 1}\left(\Gamma^{*}\right) \subsetneq \mathcal{F} \ell^{\infty, 2}\left(\Gamma^{*}\right) \subseteq W^{-1,2}(F)$.

In any case, as long as we use a Neumann series construction of the resolvent, any Banach space of potentials $E \supseteq \mathcal{F} \ell^{\infty, 1}\left(\Gamma^{*}\right)$ for which the resultant resolvent is compact on $L^{2}(F)$ will have the property that $E \cap L^{1}(F)=L^{1,1 ; 1}(F)$ due to the theorem of Stein mentioned above, that is, no new integrable potentials can be gained without significant change in our analytical toolchain.

On a more technical note, the Neumann series construction requires norm localisation. In section 2.8 , we have generalised the standard approach to all absolutely continuous Banach function spaces on $F$. This appears to be a new result, though such technical matters are seldom spoken about explicitly, and we may thus have overlooked an instance where it was done before. In our case, it is somewhat more interesting than normal, for, while the result of the generalisation is quite straightforward, namely the localisation functionals being both translation-invariant and norms, we did not succeed in creating a single suitable localisation for Banach function spaces on $\Gamma^{*}$ with these two properties. Instead, we developed two localisations. The $A$-localised norm is a norm, but it is not translation-invariant. The $N$-localised quasi-norm is not a norm, but it has translation-invariance (and is even rearrangement-invariant, something which is not true for the $r$-localised norms for spaces on $F$ ). In the proof of lemma 3.3.6, we were able to use both localisations simultaneously to exploit their respective properties when necessary. 


\subsection{Fermi curve asymptotics}

Many results of chapter 4 are new by virtue of chapter 3 alone, that is, they are straightforward generalisations of well-known theorems to $\mathcal{F} \ell^{\infty, 1}\left(\Gamma^{*}\right)$-potentials, without any new proof techniques employed, and with, in fact, proofs that are often vernacularly identical to their standard counterparts. Therefore, we shall skip those standard generalisations in our discussion, merely mentioning again that we arrive at the expected asymptotic decomposition of the Fermi curve into three parts, as in [FKT03.

In section 4.4. we gave Schrödinger operators with constant potentials a special treatment. The constant part (that is, the zeroth Fourier coefficient) of the potential plays a special role in the asymptotic theory of the Fermi curve, as can be seen in section 4.5. In particular, it interferes with the analysis of the perturbed Fourier transform. Many authors simply assume the zeroth Fourier coefficient of the potential to be zero. This is acceptable in a setting where arbitrary eigenvalues $\lambda$ are allowed, as setting $\widehat{u}(0)=0$ merely causes a shift in the energy spectrum. In our case however, where we set $\lambda=0$, we must provide for the case $\widehat{u}(0) \neq 0$. Absorbing $\widehat{u}(0)$ into the boundary condition appears to be the natural solution to get rid of the Fourier coefficient otherwise. This is especially true in the context of asymptotic analysis, where the multiplicative quantity $\xi\left(\widehat{u}_{0}, \kappa\right)$, which is a measure of the influence of $\widehat{u}(0)$ on the Fermi curve at distance roughly $\|\kappa\|$ from the origin, converges to one as $\|\kappa\| \rightarrow \infty$.

Section 4.5 contains a mixture of standard analytic techniques and some new techniques developed specifically for (quasi-)Banach function spaces, $\ell^{\infty, 1}\left(\Gamma^{*}\right)$ in particular. For one, we generalised Bochner and tensor product theory to Banach function spaces. Although simple and straightforward, this result appears to be new. Furthermore, we introduced some methods of estimation specifically for the space $\ell^{\infty, 1}\left(\Gamma^{*}\right)$ in proposition 4.5 .35 and lemmata 4.5.39, 4.5.40 and 4.5.41. They probably do not generalise well to spaces other than $\ell^{\infty, 1}\left(\Gamma^{*}\right)$.

The principal new results of section 4.5 are as expected: the perturbed Fourier transform of the potential resides in the same space as the Fourier transform of the potential itself, namely in the separable space $\ell^{\infty, 1}\left(\Gamma_{\delta}^{*}\right)$. In particular, the finite-type potentials are dense in $\mathcal{F} \ell^{\infty, 1}\left(\Gamma^{*}\right)$. Such a density result is typical in the theory of integrable systems, see for example [MO80] and Mar86, chapter 4]. Our final result is the introduction of parameters proportional to the size of the Fermi curve handles, namely the asymptotic moduli of Fermi curves, which live in a submanifold of an $\ell^{1}$-Banach manifold. This asymptotically solves the moduli problem for Fermi curves of two-dimensional, periodic Schrödinger operators. For a full solution of the moduli problem in this case, it is necessary to combine our result with a solution of the moduli problem for the compact part of the Fermi curve. Given the extant results for Fermi curves of finite genus, this is not expected to be too complicated.

\subsection{Where to go from here}

Towards a full solution of the inverse Schrödinger problem, there is still much to be done.

Now that we have asymptotically solved the Fermi moduli problem, the obvious next step is to do likewise for the Fermi isospectral problem. For simplicity, 
let us look at our model moduli calculated in 4.5.59

$$
\widetilde{t}(\nu)=\frac{\widehat{u}(u, \nu) \widehat{u}(u,-\nu)}{\|\nu\|^{2} \xi\left(\widehat{u}_{0}, \nu\right)} .
$$

Let $(\widehat{v}(u, \nu))_{\nu \in \Gamma_{\delta}^{*}}$ be a sequence such that the transformation

$$
\widehat{u}(u, \cdot) \mapsto \widehat{u}(u, \cdot) \widehat{v}(v, \cdot)
$$

does not alter the moduli. For our Fermi curve model, this means that $\widehat{v}(u, \nu)$ may be arbitrary at unsplit double points, and $\widehat{v}(u, \nu) \widehat{v}(u,-\nu)=1$ otherwise. In the generic case (no unsplit double points), the set of all possible $\widehat{v}$ yields a free abelian group isomorphic to $\left(\mathbb{C}^{\times}\right)^{\Gamma_{\delta}^{*}} /\{ \pm 1\}$ which acts freely and continuously on the perturbed Fourier transform by multiplication. Since the space of perturbed Fourier transforms of potentials is locally isomorphic to the space of regular Fourier transforms (see the proof of corollary 4.5.51), we expect the orbits of the action of this group to yield isospectral sets for our Fermi curve model. The same should be possible for the actual Fermi curve by passing to it from the model through a perturbation process as in subsection 4.5.3.

If we restrict ourselves to real-valued potentials, the Fourier transform gains the property that $\widehat{u}(\nu)$ is the complex conjugation of $\widehat{u}(-\nu)$. Hence, in this case we expect that the base group $\mathbb{C}^{\times}$appearing above is replaced by its compact subgroup of phases $S^{1} \leq \mathbb{C}^{\times}$. By Tychonoff's theorem, the resulting group is then also compact. Hence, the resulting isospectral sets should fit into a set of type $B_{C}^{N}$.

The next step from solving the inverse problem for Fermi curves would be the generalisation to Bloch varieties, that is, we no longer fix the eigenvalue $\lambda$. By 4.1.4, the Bloch variety can be seen as a stack of Fermi curves indexed by $\lambda$, or, given section 4.4 , by the zeroth Fourier coefficient of a single otherwise constant potential. Hence locally, the solution of the moduli problem for the Fermi curves can be used to solve the same problem for the Bloch varieties. Double points then become "double lines", etc. However, as the zeroth Fourier coefficient can grow arbitrary large, we cannot fit the potentials $u-\lambda$ into a bounded set like $B_{C}^{N}$ simultaneously for all $\lambda \in \mathbb{C}$. This simply means that, even asymptotically, we can no longer treat each handle separately when we try to solve the isospectral problem. Instead, altering $\lambda$ may cause handles to run into each other, adding relations to the free abelian isospectral group. For example, the formula 4.4 .2 allows us to calculate for which $\widehat{u}_{0}$ (and hence for which $\lambda$, given some fixed potential $u$ ) the double lines intersect. It turns out that for generic lattices, each node (intersection) is met by three lines. For arbitrary potentials, when the double points split, the double lines become double cylinders (in the $S^{1}$-case) representing the isospectral flow. Taking the junction of half-cylinders at each node, we expect the relations added to the isospectral group per node to be a Kirchhoff type zero sum law. At the same time, the size of the new junction defines a new modulus.

So far, we have only talked about generalisations of the two-dimensional theory. Once the inverse Schrödinger problem has been solved in its entirety in two dimensions, it is natural to ask for a solution in higher dimensions, especially for the "real world" case $d=3$. In [ERT84, KT90, Sch96, methods are developed to solve the isospectral problem. In particular, a Bloch variety of a potential $u$ in $d$ dimensions contains, in certain "infinite energy" limits, lower-dimensional 
Bloch varieties for potentials of certain "directional averages" of $u$. All Bloch varieties in dimensions lower than $d$ can be recovered this way. In order to give meaning to the phrase "infinite energy", the Bloch variety is embedded into a projective space. For the definition of "directional average", take a subspace $K \leq$ $\mathbb{R}^{d}$ that is compatible with the dual period lattice, that is, the dimension of $\bar{K}$ equals the rank of $\Gamma^{*} \cap K$. The restricted Fourier transform $\left.\widehat{u}\right|_{\Gamma^{*} \cap K}$ then gives rise to a potential $u_{K}$ which is the average of $u$ restricted to $K^{\perp}$, that is, the directions orthogonal to $K$. These results should be attempted to be generalised to larger spaces of potentials.

Of course, being able to extract the lower-dimensional Bloch varieties from higher dimensional ones does not mean that the same is possible the other way round. Indeed, there is no reason for the existence of such a "holographic principle". Nevertheless, the above method should be helpful in solving the isospectral problem, at least in the case $d>2$, for at larger dimensions the isospectral sets should already have become so small that the lattice symmetries completely generate them. In other words, the isospectral sets are minimal, and with lower-dimensional Bloch varieties contained in the Bloch variety, they cannot become larger again. The moduli problem, on the other hand, is probably not as easy.

Finally, a further avenue of generalisation is to solve the inverse problem for a different system than the time-independent Schrödinger equation, such as the time-dependent Schrödinger equation, or Schrödinger equations with a vector potential describing a magnetic field. 


\section{Appendix A}

\section{Additional function space theory}

In chapter 2 we already covered the function space theory required for the main part of this work. In this appendix, we shall only include some further addenda required for appendix B

\section{A.1 Lorentz-Karamata spaces}

In this section, we shall generalise the Lorentz-Zygmund spaces introduced in section 2.4 to the Lorentz-Karamata space ${ }^{1}$. We need this generalisation only for the case $X=\Gamma^{*}$, and there only to remove the slight constraints on convergence speed that Lorentz-Zygmund spaces still impose. To keep things simple, we shall therefore introduce Lorentz-Karamata spaces only for $X=\Gamma^{*}$. We begin with a generalisation of the logarithmic-power sequences encountered in the Lorentz-Zygmund quasi-norms.

Definition A.1.1 (Karamata sequences). A positively-valued sequence $\left(b_{n}\right)_{n \in \mathbb{N}}$ is called a Karamata sequence if it is varying more slowly than a power, that is, for all $\epsilon>0$ there are constants $C_{1}, C_{2}>0$, a non-increasing sequence $\left(a_{n}\right)_{n \in \mathbb{N}}$, and a non-decreasing sequence $\left(c_{n}\right)_{n \in \mathbb{N}}$, both with values in $\mathbb{R}_{>0}$, such that

$$
C_{1} a_{n} \leq n^{-\epsilon} b_{n} \leq C_{2} a_{n}, \quad C_{1} c_{n} \leq n^{\epsilon} b_{n} \leq C_{2} c_{n}
$$

for all $n \in \mathbb{N}$.

The generalisation of the Lorentz-Zygmund quasi-norms is now straightforward:

Definition A.1.2 (Lorentz-Karamata quasi-norms). For $0<p \leq \infty, 0<a \leq$ $\infty$ and Karamata sequences $\left(b_{n}\right)_{n \in \mathbb{N}}$, we define the Lorentz-Karamata quasi-

\footnotetext{
${ }^{1}$ Lorentz-Karamata spaces are named in honour of J. Karamata, whose research included function growth. They appear to have been introduced by J. S. Neves in his doctoral dissertation. Unfortunately, neither this dissertation nor the resulting publication Nev02 were available to us. Therefore, our account follows [EE04, 3.4.3].
} 
norms $\|\cdot\|_{p, a ; b}$ on $\mathfrak{M}\left(\Gamma^{*}\right)$ by

$$
\|f\|_{p, a ; b}:= \begin{cases}\left(\sum_{n=1}^{\infty}\left(n^{1 / p} b_{n} f^{\star}(n)\right)^{a} n^{-1}\right)^{1 / a}, & \text { if } 0<a<\infty, \\ \sup _{n=1}^{\infty} n^{1 / p} b_{n} f^{\star}(n), & \text { if } a=\infty .\end{cases}
$$

For $b_{n}=(1+\log n)^{\alpha}$, one obtains the Lorentz-Zygmund spaces. Again, it is easy to see that for $a \neq \infty$, the resulting Lorentz-Karamata spaces $\ell^{p, a ; b}\left(\Gamma^{*}\right)$ are Banach spaces whenever $n^{a / p-1} b_{n}^{a}$ is non-increasing. In fact, we have

Theorem A.1.3. Let $1<q<p \leq \infty$ and let $b$ be a non-decreasing, divergent Karamata sequence. Then $\ell^{p, 1 ; b}\left(\Gamma^{*}\right)$ is, possibly after switching to an equivalent quasi-norm, a Banach space and we have the continuous embeddings

$$
\ell^{q}\left(\Gamma^{*}\right) \subseteq \ell^{p, 1 ; b}\left(\Gamma^{*}\right) \subseteq \ell^{p, 1}\left(\Gamma^{*}\right) .
$$

Proof. The continuity of the embeddings follows immediately from the definition of the Lorentz-Zygmund, resp. Lorentz-Karamata quasi-norms. Now, EE04, Lemma 3.4.34 (i)] together with the integral convergence criterion implies that the quasi-norm of $\ell^{p, 1 ; b}\left(\Gamma^{*}\right)$ is equivalent to

$$
f \mapsto b_{1}\|f\|_{\infty}+\sum_{n=1}^{\infty} n^{1 / p} b_{n} f^{\star \star}(n) n^{-1} .
$$

Since $\ell^{p, 1 ; b}\left(\Gamma^{*}\right) \subseteq \ell^{\infty}\left(\Gamma^{*}\right)$ continuously, this is in turn equivalent to

$$
f \mapsto \sum_{n=1}^{\infty} n^{1 / p} b_{n} f^{\star \star}(n) n^{-1} .
$$

But this is a Banach function norm by proposition 2.3.11.

Let us now state two stability statements for Karamata sequences.

Proposition A.1.4 ([EE04, 3.4.33 (i)]). If $b$ is a Karamata sequence, so is $b^{r}$, for all $r \in \mathbb{R}$.

Proposition A.1.5. Let $f \in \ell^{\infty, 1}\left(\Gamma^{*}\right)$ such that $f^{\star}$ is a Karamata sequence. Then the sequence $\left(b_{n}\right)_{n \in \mathbb{N}}$ defined by

$$
b_{n}:=\sum_{k=n}^{\infty} \frac{f^{\star}(k)}{k}
$$

is a non-increasing Karamata sequence with $\lim _{n \rightarrow \infty} b_{n}=0$.

Proof. That $\left(b_{n}\right)_{n \in \mathbb{N}}$ is a Karamata sequence follows from [EE04, 3.4.33 (iv)]. Since $f^{\star}$ is non-negative, that sequence is non-increasing. The limit of zero is obvious.

The following theorem is the main reason why we introduced the LorentzKaramata spaces in the first place. It will become important in appendix B. 
Theorem A.1.6. As a set, the Lorentz space $\ell^{\infty, 1}\left(\Gamma^{*}\right)$ is given by

$$
\ell^{\infty, 1}\left(\Gamma^{*}\right)=\bigcup_{b} \ell^{\infty, 1 ; b}\left(\Gamma^{*}\right),
$$

where the union runs over all non-decreasing, divergent Karamata sequences.

Proof. The inclusion " $\supseteq$ " follows from theorem A.1.3. Now let $f \in \ell^{\infty, 1}\left(\Gamma^{*}\right)$. The rearrangement $f^{\star}$ is non-increasing, so if $f^{\star}$ is not a Karamata sequence, then $f^{\star}$ falls, at times, faster than some power, and can thus be majorised by a Karamata sequence. It is therefore sufficient to investigate the case where $f^{\star}$ is a Karamata sequence. In this case, proposition A.1.5 implies that

$$
b_{n}:=\sum_{k=n}^{\infty} \frac{f^{\star}(k)}{k}
$$

defines a non-increasing Karamata sequence $b$ converging to zero. Hence, by proposition A.1.4 $1 / \sqrt{b}$ is a non-decreasing, divergent Karamata sequence. Now,

$$
\sum_{n=1}^{\infty} \frac{f^{\star}(n)}{n \sqrt{b_{n}}}<\infty
$$

by a classical convergence test due to Dini (see e.g. [Kno64, Satz 175.4]). Hence, $f \in \ell^{\infty, 1 ; 1 / \sqrt{b}}\left(\Gamma^{*}\right)$. 


\section{Appendix B}

\section{Extension of the Schrödinger theory to Dirac operators - a failed attempt}

The Dirac equation is the relativistic counterpart of the Schrödinger equation. In this work, we shall treat only the two-dimensional Dirac equation, that is, $d=2$, corresponding to one time and one space coordinat 1 . Physicists use the (four-dimensional) Dirac equation to describe high-energy behaviour such as that of electrons bound to highly charged atomic nuclei more accurately than with the Schrödinger equation. Since this is a work about Schrödinger operators, we are rather interested in the zero energy case $\lambda=0$. In section B.2, we shall prove the physically expected result that the spectral data of Schrödinger and Dirac operators coincide at $\lambda=0$ for each fixed Schrödinger potential and corresponding Dirac potentials. Initially, Dirac operators appeared to be preferable over Schrödinger operators due to their slightly higher flexibility in choice of potential and boundary conditions, and the fact that Dirac operators are first-order differential operators, while the Schrödinger operator is second order. The former point in particular leads to a symmetry in the Fermi curves of Dirac operators which the corresponding Schrödinger Fermi curves do not have. (On the other hand, Dirac Fermi curves generally do not have the point symmetry of Schrödinger Fermi curves.)

In order to truly generalise the Schrödinger case, it is necessary to introduce a certain symmetry breaking in the Dirac potentials. While this works quite well at first, it causes problems later. Namely at the point where the perturbed Fourier transform (the analog of $\widehat{u}(u, \pm \nu)$ from the Schrödinger case) is defined, the dissimilar spaces of the potentials mix in an apparently irreconcilable way. Therefore, we feel compelled to brand this attempt at arriving at a partial

\footnotetext{
${ }^{1}$ While it is customary in relativistic physics to to denote the time coordinate by $x_{0}$ and the space coordinate by $x_{1}$ in a Minkowski space, we shall remain in an a priori Euclidean picture and use the indices 1 and 2 . These two coordinates are not to be confused with the two components of a solution of the Dirac equation, for which we shall use the same indices.
} 
solution of the moduli problem as failed. Without the symmetry breaking, when the spaces of potentials are equal, the theory works quite well (see [Sch02]).

Since there is a certain aesthetic beauty to the Dirac theory, we would like to present it here as an appendix, at least to the point of failure, if only to serve as an example that even late in a mathematical development, an impasse may occur.

\section{B.1 Dirac operators}

In this section we shall derive properties of Dirac operators and their resolvents just as we did in chapter 3 for Schrödinger operators. However, it must be noted that passing to the Dirac operator introduces certain technical problems as regards Riesz-Schauder theory which destroy the uniformness of some results from the Schrödinger theory. In what follows, we shall explain these shortcomings in detail whenever they arise.

\section{B.1.1 Dirac operators on the torus}

By the Dirac equation, we mean the matrix equation

$$
\left(\begin{array}{cc}
v & \partial \\
-\bar{\partial} & w
\end{array}\right)\left(\begin{array}{l}
\psi_{1} \\
\psi_{2}
\end{array}\right)=\lambda\left(\begin{array}{l}
\psi_{1} \\
\psi_{2}
\end{array}\right)
$$

Recall that the two differential operators $\partial$ and $\bar{\partial}$ occurring here are the Wirtinger operators, which we introduced in section 4.2 Unlike the Schrödinger equation 3.1.1), the Dirac equation contains two potentials $v, w: \mathbb{R}^{2} \rightarrow \mathbb{C}$. The solution $\psi:=\left(\psi_{1}, \psi_{2}\right)^{t}$ also has two components. As in the Schrödinger case, $\lambda$ denotes the eigenvalue.

The boundary conditions for the Dirac equation (B.1.1) are similar to the Schrödinger case. Again, let $\Gamma \subseteq \mathbb{R}^{2}$ be a non-degenerate geometric lattice, then we demand that

$$
v(x+\gamma)=v(x), \quad w(x+\gamma)=w(x), \quad \text { for all } x \in \mathbb{R}^{2}, \gamma \in \Gamma .
$$

For the solution $\psi$, we demand, in complete analogy to (3.1.3), that for the boundary condition $k \in \mathbb{C}^{2}$, we have

$$
\psi(x+\gamma)=e^{2 \pi i(k \mid \gamma)} \psi(x) \quad \text { for all } x \in \mathbb{R}^{2}, \gamma \in \Gamma .
$$

As is the case for the Schrödinger equation, we shall want to hide the boundary condition within the operator and formulate a single Dirac equation on $F:=$ $\mathbb{R}^{2} / \Gamma$. The Wirtinger operators with boundary conditions $\partial_{k}$ and $\bar{\partial}_{k}$ introduced in 4.2.1) accomplish just that, as we presently shall see: again, we have (cf. proposition 3.1.6

Proposition B.1.4. For all $k \in \mathbb{C}^{2}$, the operators $\partial_{k}$ and $\bar{\partial}_{k}$ formally map the space of $\Gamma$-periodic functions on $\mathbb{R}^{2}$ into itself.

For convenience, we introduce the notation

$$
D(k, v, w):=\left(\begin{array}{cc}
v & \partial_{k} \\
-\bar{\partial}_{k} & w
\end{array}\right)
$$


for the Dirac operator. We then have the Dirac equation

$$
D(k, v, w) \psi=\lambda \psi
$$

entirely on $F$. Lemma 3.1 .9 also holds in the Dirac case as its truth only depends on 3.1.3 and not on the operator itself. Therefore, we can prove in analogy to theorem 3.1.10.

Theorem B.1.6. The quantities $k, v, w, \psi$ and $\lambda$ fulfil the equations (B.1.1), B.1.2 and B.1.3 if and only if

$$
D(k, v, w) e^{2 \pi i(k \mid x)} \psi(x)=\lambda e^{2 \pi i(k \mid x)} \psi(x) .
$$

Proof. From 4.2.1), we derive

$$
\begin{aligned}
\partial e^{2 \pi i(k \mid x)} \psi_{2}(x) & =e^{2 \pi i(k \mid x)} \partial_{k} \psi_{2}(x), \\
-\bar{\partial} e^{2 \pi i(k \mid x)} \psi_{1}(x) & =e^{2 \pi i(k \mid x)}\left(-\bar{\partial}_{k} \psi_{1}(x)\right) .
\end{aligned}
$$

Hence, the claim follows as in 3.1.11.

Therefore, we need, as in the Schrödinger case, only consider the Dirac equation B.1.5. Note that much more obviously than the Schrödinger equation, B.1.5 readily admits the generalisation

$$
\left(\begin{array}{cc}
v & \partial_{k} \\
-\bar{\partial}_{k^{\prime}} & w
\end{array}\right) \psi=\lambda \psi,
$$

where $k, k^{\prime} \in \mathbb{C}^{2}$ are distinct in general. We shall investigate this possibility later in order to derive a certain translation symmetry.

\section{B.1.2 Resolvents of free Dirac operators}

For convenience, we denote the general resolvent of the Dirac operator by

$$
R(\lambda, k, v, w):=\left(\lambda-\left(\begin{array}{cc}
v & \partial_{k} \\
-\bar{\partial}_{k} & w
\end{array}\right)\right)^{-1} .
$$

In this subsection, we would like to define the free resolvent

$$
R(\lambda, 0,0,0)=\left(\begin{array}{cc}
\lambda & -\partial_{0} \\
\bar{\partial}_{0} & \lambda
\end{array}\right)^{-1}
$$

for $\lambda \in(-\infty,-1] i$. For technical reasons, however, it will be convenient to include boundary conditions right away. The initial domain for $\lambda$ in the resolvents with boundary conditions will then be dependent on set of boundary conditions given.

Theorem B.1.7. For all bounded $V \subseteq \mathbb{C}^{2}$ there is a $\lambda_{0} \in(-\infty,-1] i$ such that the resolvent $R(\lambda, k, 0,0)$ is defined and maps $\mathcal{F} \ell^{\infty, 1}\left(\Gamma^{*}\right) \times \mathcal{F} \ell^{2,1}\left(\Gamma^{*}\right)$ boundedly into $\mathcal{F} \ell^{1}\left(\Gamma^{*}\right) \times \mathcal{F} \ell^{2,1}\left(\Gamma^{*}\right)$ for all $\lambda / i \leq \lambda_{0} / i$ and all $k \in V$. While the choice of $\lambda_{0}$ depends on $V$, the bound on the resolvent can be chosen independently of $V$ and $\lambda$. 
Proof. Since we are using Fourier spaces, we need to examine the Fourier transform of $R(\lambda, k, 0,0)$. The operator

$$
\left(\begin{array}{cc}
\lambda & -\partial_{k} \\
\bar{\partial}_{k} & \lambda
\end{array}\right)
$$

is defined on $\mathfrak{S}(F) \times \mathfrak{S}(F)$ and its Fourier transform $x \mapsto \kappa$ is the multiplication with

$$
\left(\begin{array}{cc}
\lambda & -\pi i\left(\kappa_{1}+k_{1}\right)-\pi\left(\kappa_{2}+k_{2}\right) \\
\pi i\left(\kappa_{1}+k_{1}\right)-\pi\left(\kappa_{2}+k_{2}\right) & \lambda
\end{array}\right) .
$$

Therefore, the Fourier transform of $R(\lambda, k, 0,0)$ is the multiplication with

$$
\left(\begin{array}{cc}
\frac{\lambda}{\lambda^{2}-\pi^{2}(\kappa+k)^{2}} & \frac{\pi i\left(\kappa_{1}+k_{1}\right)+\pi\left(\kappa_{2}+k_{2}\right)}{\lambda^{2}-\pi^{2}(\kappa+k)^{2}} \\
\frac{-\pi i\left(\kappa_{1}+k_{1}\right)+\pi\left(\kappa_{2}+k_{2}\right)}{\lambda^{2}-\pi^{2}(\kappa+k)^{2}} & \frac{\lambda}{\lambda^{2}-\pi^{2}(\kappa+k)^{2}}
\end{array}\right) .
$$

The diagonal entry $\lambda /\left(\lambda^{2}-\pi^{2}(\kappa+k)^{2}\right)$ maps $\ell^{\infty, 1}\left(\Gamma^{*}\right)$ boundedly into $\ell^{1}\left(\Gamma^{*}\right)$ as in the proof of theorem 3.2.5 if we choose $\lambda_{0}$ such that $\left|\lambda^{2}-\pi^{2}(\kappa+k)^{2}\right| \geq 1$ for all $\lambda / i \leq \lambda_{0} / i$, all $k \in V$ and all $\kappa \in \Gamma^{*}$. This is possible due to lemma 3.2 .15 The same diagonal entry maps $\ell^{2,1}\left(\Gamma^{*}\right)$ into an obviously much better space than $\ell^{2,1}\left(\Gamma^{*}\right)$. Since $|\lambda| /\left|\lambda^{2}-\pi^{2}(\kappa-k)^{2}\right|$ (with proper choice of $\lambda_{0}$ ) decreases monotonously as $|\lambda| \nearrow \infty$, no $\lambda$-dependence is introduced into the bound. Now, let $g(\kappa)$ be one of the off-diagonal entries, then

$$
g^{\star}(n)=\pi \frac{\left|\kappa_{n}\right|}{\left|\lambda^{2}-\pi^{2}\left(\kappa_{n}+k\right)^{2}\right|},
$$

where $\left(\kappa_{n}\right)_{n \in \mathbb{N}}$ is an arrangement such that $\left|\left(\kappa_{n}+k\right)^{2}\right| \geq\left|\left(\kappa_{n^{\prime}}+k\right)^{2}\right|$ whenever $n \geq n^{\prime}$, just as in (3.2.7). Given that $|g(\kappa)|$ decreases monotonously as $|\kappa| \nearrow \infty$, and by our choice of $\lambda_{0}$, by similar reasoning as in the proof of theorem 3.2 .5 . we arrive at the estimate

$$
\frac{C_{1} \sqrt{n-1}}{\left|\left(\lambda-\lambda_{0}\right)^{2}\right|+C_{2}(n-1)} \leq g^{\star}(n) \leq \frac{C_{3} \sqrt{n-1}}{\left|\left(\lambda-\lambda_{0}\right)^{2}\right|+C_{4}(n-1)}
$$

where $C_{1}, C_{2}, C_{3}, C_{4}>0$ with $C_{4}<1$ are constants depending only on $\Gamma^{*}$. Now let $f \in \ell^{2,1}\left(\Gamma^{*}\right)$, then by the Hardy-Littlewood inequality (theorem 2.3.6):

$$
\begin{aligned}
& \sum_{\kappa \in \Gamma^{*}}|g(\kappa) f(\kappa)| \leq \sum_{n=1}^{\infty} g^{\star}(n) f^{\star}(n) \leq \sum_{n=1}^{\infty} \frac{f^{\star}(n) C_{3} \sqrt{n-1}}{\left|\left(\lambda-\lambda_{0}\right)^{2}\right|+C_{4}(n-1)} \\
& \leq \frac{C_{3}}{C_{4}} \sum_{n=1}^{\infty} \frac{f^{\star}(n)}{\sqrt{n}}=\frac{C_{3}}{C_{4}}\|f\|_{2,1},
\end{aligned}
$$

so multiplication with $g$ maps $\ell^{2,1}\left(\Gamma^{*}\right)$ boundedly into $\ell^{1}\left(\Gamma^{*}\right)$, with a bound independent of $\lambda$. Since $n \mapsto 1 / \sqrt{n}$ is monotonically decreasing, we can make a similar calculation for the case $f \in \ell^{\infty, 1}\left(\Gamma^{*}\right)$ due to Hardy's lemma (proposition 2.3.5:

$$
\sum_{n=1}^{\infty} \frac{(g f)^{\star}(n)}{\sqrt{n}} \leq \sum_{n=1}^{\infty} \frac{g^{\star}(n) f^{\star}(n)}{\sqrt{n}} \leq \frac{C_{3}}{C_{4}} \sum_{n=1}^{\infty} \frac{f^{\star}(n)}{\sqrt{n} \sqrt{n}}=\frac{C_{3}}{C_{4}}\|f\|_{\infty, 1},
$$

so multiplication with $g$ also maps $\ell^{\infty, 1}\left(\Gamma^{*}\right)$ boundedly into $\ell^{2,1}\left(\Gamma^{*}\right)$, with a bound independent of $\lambda$. This proves the theorem. 
The choice of spaces $\mathcal{F} \ell^{\infty, 1}\left(\Gamma^{*}\right) \times \mathcal{F} \ell^{2,1}\left(\Gamma^{*}\right) \rightarrow \mathcal{F} \ell^{1}\left(\Gamma^{*}\right) \times \mathcal{F} \ell^{2,1}\left(\Gamma^{*}\right)$ may seem somewhat odd at first glance but is (almost) right on tune with the spaces we shall choose for our potentials $v, w$ in the next subsection, at least as far as the possible range of rearrangement-invariant Banach spaces is concerned (cf. theorem 2.3.3). But let us prove the following compactness result first.

Theorem B.1.9. Let $1 \leq p_{1}, q_{1}, p_{2}, q_{2} \leq \infty$ such that

$$
\begin{array}{ll}
\frac{1}{p_{2}}-\frac{1}{p_{1}}<1, & \frac{1}{q_{2}}-\frac{1}{q_{1}}<1, \\
\frac{1}{p_{2}}-\frac{1}{q_{1}}<\frac{1}{2}, & \frac{1}{q_{2}}-\frac{1}{p_{1}}<\frac{1}{2} .
\end{array}
$$

Then for all bounded $V \subseteq \mathbb{C}^{2}$ there is a $\lambda_{0} \in(-\infty,-1] i$ such that the resolvent $R(\lambda, k, 0,0)$ is defined and maps $\mathcal{F} \ell^{p_{1}, 1}\left(\Gamma^{*}\right) \times \mathcal{F} \ell^{q_{1}, 1}\left(\Gamma^{*}\right)$ compactly into the space $\mathcal{F} \ell^{p_{2}, 1}\left(\Gamma^{*}\right) \times \mathcal{F} \ell^{q_{2}, 1}\left(\Gamma^{*}\right)$, for all $\lambda / i \leq \lambda_{0} / i$ and all $k \in V$. Moreover, let $b$ be a non-decreasing, divergent Karamata sequence, then $R(\lambda, k, 0,0)$ maps $\mathcal{F} \ell^{\infty, 1 ; b}\left(\Gamma^{*}\right) \times \mathcal{F} \ell^{2,1 ; \sqrt{b}}\left(\Gamma^{*}\right)$ compactly into $\mathcal{F} \ell^{1}\left(\Gamma^{*}\right) \times \mathcal{F} \ell^{2,1 ; \sqrt{b}}\left(\Gamma^{*}\right)$. In all these cases, the bound on $R(\lambda, k, 0,0)$ vanishes as $|\lambda| \rightarrow \infty$.

Proof. The proof of the first part is similar to the proof of theorem 3.2.10. Using the sequence of finite-rank operators $\left(P_{m}\right)_{m \in \mathbb{N}}$ from that proof, we get, for

$$
g_{1}(\kappa):=\frac{\lambda}{\lambda^{2}-\pi^{2}(\kappa+k)^{2}},
$$

the estimate

$$
\left\|g_{1} f-P_{m} g_{1} f\right\|_{s, 1} \leq \sup _{n \in \mathbb{N}} \frac{\left|\lambda-\lambda_{0}\right| n^{1 / s-1 / r}}{\left|\lambda-\lambda_{0}\right|^{2}+C(n+m-1)}\|f\|_{r, 1}
$$

for a suitable $C>0$ and all $1 \leq r, s \leq \infty$. This supremum vanishes for $m \rightarrow \infty$ whenever $1 / s-1 / r<1$. This is the case for $(r, s)=\left(p_{1}, q_{1}\right)$ and $(r, s)=\left(p_{2}, q_{2}\right)$. For

$$
g_{2}(\kappa):=\frac{ \pm \pi i\left(\kappa_{1}+k_{1}\right)+\pi\left(\kappa_{2}+k_{2}\right)}{\lambda^{2}-(\kappa+k)^{2}}
$$

we get the estimate

$$
\left\|g_{2} f-P_{m} g_{2} f\right\|_{s, 1} \leq \sup _{n \in \mathbb{N}} \frac{C^{\prime} \sqrt{n+m-1} n^{1 / s-1 / r}}{\left|\lambda-\lambda_{0}\right|^{2}+C(n+m-1)}\|f\|_{r, 1}
$$

for suitable $C, C^{\prime}>0$ and all $1 \leq r, s \leq \infty$. This time, the supremum vanishes for $m \rightarrow \infty$ whenever $1 / s-1 / r<1 / 2$, which is the case for $(r, s)=\left(p_{1}, q_{2}\right)$ and $(r, s)=\left(q_{1}, p_{2}\right)$.

Let's turn to the cases where Lorentz-Karamata spaces are involved. We get

$$
\begin{aligned}
\left\|g_{1} f-P_{m} g_{1} f\right\|_{1} & \leq \sup _{n \in \mathbb{N}} \frac{\left|\lambda-\lambda_{0}\right| n}{\left(\left|\lambda-\lambda_{0}\right|^{2}+C(n+m-1)\right) b_{n}}\|f\|_{\infty, 1 ; b}, \\
\left\|g_{2} f-P_{m} g_{2} f\right\|_{1} & \leq \sup _{n \in \mathbb{N}} \frac{C^{\prime} \sqrt{n+m-1} \sqrt{n}}{\left(\left|\lambda-\lambda_{0}\right|^{2}+C(n+m-1)\right) \sqrt{b_{n}}}\|f\|_{2,1 ; \sqrt{b}}, \\
\left\|g_{2} f-P_{m} g_{2} f\right\|_{2,1 ; \sqrt{b}} & \leq \sup _{n \in \mathbb{N}} \frac{C^{\prime} \sqrt{n+m-1} \sqrt{n}}{\left(\left|\lambda-\lambda_{0}\right|^{2}+C(n+m-1)\right) \sqrt{b_{n}}}\|f\|_{\infty, 1 ; b}, \\
\left\|g_{2} f-P_{m} g_{2} f\right\|_{2,1 ; \sqrt{b}} & \leq \sup _{n \in \mathbb{N}} \frac{\left|\lambda-\lambda_{0}\right|}{\left(\left|\lambda-\lambda_{0}\right|^{2}+C(n+m-1)\right)}\|f\|_{2,1 ; \sqrt{b}} .
\end{aligned}
$$


The last of these suprema clearly vanishes as $m \rightarrow \infty$. For the remainder, we prove in a model calculation that

$$
\lim _{m \rightarrow \infty} \sup _{n \in \mathbb{N}} \frac{a_{n} n}{n+m}=0
$$

for all non-increasing sequences $\left(a_{n}\right)_{n \in \mathbb{N}}$ converging to zero. But this already follows from lemma 4.5.39 and lemma 4.5.41. The above suprema can be estimated against the model calculation. For similar reasons, the norms vanish as $|\lambda| \rightarrow \infty$.

\section{B.1.3 Resolvents of general Dirac operators}

In this subsection, we shall add potentials $v, w$ and arbitrary $\lambda$ in close analogy to section 3.3. Recall that it was our goal to use the Dirac equation to describe aspects of the Schrödinger case. Therefore, we shall not take the potentials $v, w$ from one space. Instead, we will use the potential $v$ to essentially fill the role of the Schrödinger potential $u$, that is, we choose $v \in \mathcal{F} \ell^{\infty, 1}\left(\Gamma^{*}\right)$. On the other hand, it will be sufficient (and, for our purposes, necessary) to choose the potential $w$ from the much smaller space $\mathcal{F} \ell^{1}\left(\Gamma^{*}\right)$, with an upper bound on the potential at that. Occasionally, it will be necessary to make use of the fact proven in theorem A.1.6 that each $v \in \mathcal{F} \ell^{\infty, 1}\left(\Gamma^{*}\right)$ is also element of some Lorentz-Karamata Fourier space $\mathcal{F} \ell^{\infty, 1 ; b}\left(\Gamma^{*}\right)$, where $b$ is a non-decreasing, divergent Karamata sequence.

Lemma B.1.10. Let $V \subseteq \mathbb{C}^{2}$ be bounded. Then there is a $C_{2}>0$ such that for all $v_{0} \in \mathcal{F} \ell^{\infty, 1}\left(\Gamma^{*}\right)$ there is an $N \in \mathbb{N}$ and $a C_{1}>0$ with $v_{0} \in B_{C_{1}}^{N}$ as well as a $\lambda_{0} \in(-\infty,-1] i$ such that the operator

$$
\left(\begin{array}{cc}
v & 0 \\
0 & w
\end{array}\right) R(\lambda, k, 0,0)
$$

is bounded on $\mathcal{F} \ell^{\infty, 1}\left(\Gamma^{*}\right) \times \mathcal{F} \ell^{2,1}\left(\Gamma^{*}\right)$ for all $v \in B_{C_{1}}^{N}, w \in \mathcal{F} \ell^{1}\left(\Gamma^{*}\right)$ with $\|w\|_{\mathcal{F} 1}<$ $C_{2}, k \in V$ and all $\lambda \in(-\infty,-1] i$ with $\lambda / i \leq \lambda_{0} / i$. There is a norm equivalent to the standard norm on $\mathcal{F} \ell^{\infty, 1}\left(\Gamma^{*}\right) \times \mathcal{F} \ell^{2,1}\left(\Gamma^{*}\right)$ such that

$$
\left\|\left(\begin{array}{cc}
v & 0 \\
0 & w
\end{array}\right) R(\lambda, k, 0,0)\right\|<1 .
$$

The same statement, possibly with different constants $N, C_{1}, C_{2}, \lambda_{0}$, holds if one replaces $\mathcal{F} \ell^{\infty, 1}\left(\Gamma^{*}\right) \times \mathcal{F} \ell^{2,1}\left(\Gamma^{*}\right)$ with $\mathcal{F} \ell^{\infty, 1 ; b}\left(\Gamma^{*}\right) \times \mathcal{F} \ell^{2,1 ; \sqrt{b}}\left(\Gamma^{*}\right)$ for $v \in$ $B_{C_{1}}^{N} \cap \mathcal{F} \ell^{\infty, 1 ; b}\left(\Gamma^{*}\right)$ where $b$ is a non-decreasing, divergent Karamata sequence.

Proof. By theorem B.1.7 $R(\lambda, k, 0,0)$ maps $\mathcal{F} \ell^{\infty, 1}\left(\Gamma^{*}\right) \times \mathcal{F} \ell^{2,1}\left(\Gamma^{*}\right)$ boundedly into $\mathcal{F} \ell^{1}\left(\Gamma^{*}\right) \times \mathcal{F} \ell^{2,1}\left(\Gamma^{*}\right)$, and by theorem B.1.9, $R(\lambda, k, 0,0)$ maps $\mathcal{F} \ell^{\infty, 1 ; b}\left(\Gamma^{*}\right) \times$ $\mathcal{F} \ell^{2,1 ; \sqrt{b}}\left(\Gamma^{*}\right)$ boundedly into $\mathcal{F} \ell^{1}\left(\Gamma^{*}\right) \times \mathcal{F} \ell^{2,1 ; \sqrt{b}}\left(\Gamma^{*}\right)$. Therefore the operator B.1.11 maps both $\mathcal{F} \ell^{\infty, 1}\left(\Gamma^{*}\right) \times \mathcal{F} \ell^{2,1}\left(\Gamma^{*}\right)$ and $\mathcal{F} \ell^{\infty, 1 ; b}\left(\Gamma^{*}\right) \times \mathcal{F} \ell^{2,1 ; \sqrt{b}}\left(\Gamma^{*}\right)$ boundedly into themselves, since by proposition 2.4.15, we have the bounded operations

$$
\begin{aligned}
\ell^{\infty, 1}\left(\Gamma^{*}\right) * \ell^{1}\left(\Gamma^{*}\right) & \rightarrow \ell^{\infty, 1}\left(\Gamma^{*}\right), & \ell^{1}\left(\Gamma^{*}\right) * \ell^{2,1}\left(\Gamma^{*}\right) & \rightarrow \ell^{2,1}\left(\Gamma^{*}\right), \\
\ell^{\infty, 1 ; b}\left(\Gamma^{*}\right) * \ell^{1}\left(\Gamma^{*}\right) & \rightarrow \ell^{\infty, 1 ; b}\left(\Gamma^{*}\right), & \ell^{1}\left(\Gamma^{*}\right) * \ell^{2,1 ; \sqrt{b}}\left(\Gamma^{*}\right) & \rightarrow \ell^{2,1 ; \sqrt{b}}\left(\Gamma^{*}\right) .
\end{aligned}
$$


We now need to find equivalent norms on $\mathcal{F} \ell^{\infty, 1}\left(\Gamma^{*}\right)$ and $\mathcal{F} \ell^{\infty, 1 ; b}\left(\Gamma^{*}\right)$ to make the norm of B.1.11) smaller than one for suitable $v, w, \lambda$. Recall (B.1.8), the Fourier transform of $R(\lambda, k, 0,0)$. It follows that the Fourier transform of (B.1.11) is given by

$$
\left(\begin{array}{c}
f_{1} \\
f_{2}
\end{array}\right) \mapsto \frac{1}{\mu(F)}\left(\begin{array}{c}
\widehat{v} *\left(\frac{\lambda}{\lambda^{2}-\pi^{2}(\kappa+k)^{2}} f_{1}\right) \\
+\widehat{v} *\left(\frac{\pi i\left(\kappa_{1}+k_{1}\right)+\pi\left(\kappa_{2}+k_{2}\right)}{\lambda^{2}-\pi^{2}(\kappa+k)^{2}} f_{2}\right) \\
\widehat{w} *\left(\frac{-\pi i\left(\kappa_{1}+k_{1}\right)+\pi\left(\kappa_{2}+k_{2}\right)}{\lambda^{2}-\pi^{2}(\kappa+k)^{2}} f_{1}\right) \\
+\widehat{w} *\left(\frac{\lambda}{\lambda^{2}-\pi^{2}(\kappa+k)^{2}} f_{2}\right)
\end{array}\right)
$$

Assuming $f_{1} \in \ell^{\infty, 1}\left(\Gamma^{*}\right)$ (resp. $f_{1} \in \ell^{\infty, 1 ; b}\left(\Gamma^{*}\right)$ ) and $f_{2} \in \ell^{2,1}\left(\Gamma^{*}\right)$ (resp. $f_{2} \in$ $\left.\ell^{2,1 ; \sqrt{b}}\left(\Gamma^{*}\right)\right)$, we shall now estimate the norm of this operator. The $\widehat{w}$-terms certainly have norm smaller than one for $\|w\|_{\mathcal{F} 1}<C_{2}$ if $C_{2}$ is chosen small enough (in fact, $C_{2}$ can be chosen independently of $b$ ). As for the $\widehat{v}$-terms, the norm of

$$
\widehat{v} *\left(\frac{\lambda}{\lambda^{2}-\pi^{2}(\kappa+k)^{2}} f_{1}\right)
$$

is diminishable using $N$-localised quasi-norms and levelling operators quite precisely as in the proof of lemma 3.3 .6 (with $C$ replaced by $C_{1}$ ) since $\lambda$ appears quadratically in the denominator but only linearly in the numerator. The same is possible with

$$
\widehat{v} *\left(\frac{\pi i\left(\kappa_{1}+k_{1}\right)+\pi\left(\kappa_{2}+k_{2}\right)}{\lambda^{2}-\pi^{2}(\kappa+k)^{2}} f_{2}\right) .
$$

Decompose $\widehat{v}=\widehat{v}_{1}+\widehat{v}_{2}$ as before. Here, we can gratuitously estimate $f_{2}$ with the $\ell^{\infty, 1}\left(\Gamma^{*}\right)$-norm for the $\widehat{v}_{2}$ part. The expression

$$
\frac{\left|\pi i\left(\kappa_{1}+k_{1}\right)+\pi\left(\kappa_{2}+k_{2}\right)\right|}{\sqrt{\left|\lambda^{2}-\pi^{2}(\kappa+k)^{2}\right|}}
$$

is bounded, leaving a $|\lambda|$ for diminishing. The use of Lorentz-Karamata spaces is relevant only for the $\widehat{v}_{1}$-part, where the diminishing occurs through choice of suitable constants.

Proposition B.1.12. For all bounded $V \subseteq \mathbb{C}^{2}$ and all $v_{0} \in \mathcal{F} \ell^{\infty, 1}\left(\Gamma^{*}\right)$ there is $a \lambda_{0} \in(-\infty,-1] i$ such that the resolvent $R(\lambda, k, v, w)$ is defined and maps the space $\mathcal{F} \ell^{\infty, 1}\left(\Gamma^{*}\right) \times \mathcal{F} \ell^{2,1}\left(\Gamma^{*}\right)$ boundedly into $\mathcal{F} \ell^{1}\left(\Gamma^{*}\right) \times \mathcal{F} \ell^{2,1}\left(\Gamma^{*}\right)$ for all $\lambda / i \leq$ $\lambda_{0} / i, k \in V, v \in B_{C_{1}}^{N}$ and all $w \in \mathcal{F} \ell^{1}\left(\Gamma^{*}\right)$ with $\|w\|_{\mathcal{F} 1}<C_{2}$, with the same $N$, $C_{1}, C_{2}$ as in lemma B.1.10. Moreover, denote the set of applicable pairs of potentials above by $U$, then there is a non-decreasing, divergent Karamata sequence $b$ with $v_{0} \in \mathcal{F} \ell^{\infty, 1 ; b}\left(\Gamma^{*}\right)$ such that the map

$$
\begin{aligned}
&\left(U \cap \mathcal{F} \ell^{\infty, 1 ; b}\left(\Gamma^{*}\right) \times \mathcal{F} \ell^{1}\left(\Gamma^{*}\right)\right) \times V \\
& \\
& \rightarrow \mathcal{K}\left(\mathcal{F} \ell^{2,1 ; \sqrt{b}}\left(\Gamma^{*}\right) \times \mathcal{F} \ell^{2,1 ; \sqrt{b}}\left(\Gamma^{*}\right), \mathcal{F} \ell^{2,1 ; \sqrt{b}}\left(\Gamma^{*}\right) \times \mathcal{F} \ell^{2,1 ; \sqrt{b}}\left(\Gamma^{*}\right)\right) \\
&(v, w, k) \mapsto R(\lambda, k, v, w)
\end{aligned}
$$

is compact with respect to the weak topology on $U$ and the strong topologies on $V$ and $\mathcal{K}\left(\mathcal{F} \ell^{2,1 ; \sqrt{b}}\left(\Gamma^{*}\right) \times \mathcal{F} \ell^{2,1 ; \sqrt{b}}\left(\Gamma^{*}\right), \mathcal{F} \ell^{2,1 ; \sqrt{b}}\left(\Gamma^{*}\right) \times \mathcal{F} \ell^{2,1 ; \sqrt{b}}\left(\Gamma^{*}\right)\right)$ for all $\lambda \in$ $(-\infty,-1] i$ with $\lambda / i \leq \lambda_{0} / i$. Continuity is uniform with respect to $k$. 
Proof. Due to theorem A.1.6, we can find a suitable Karamata sequence $b$ with $v_{0} \in \mathcal{F} \ell^{\infty, 1 ; b}\left(\Gamma^{*}\right)$. The proof is now similar to the proof of proposition 3.3.21. By lemma B.1.10 and the Neumann theorem,

$$
1-\left(\begin{array}{cc}
v & 0 \\
0 & w
\end{array}\right) R(\lambda, k, 0,0)
$$

is boundedly invertible on $\mathcal{F} \ell^{\infty, 1}\left(\Gamma^{*}\right) \times \mathcal{F}^{2,1}\left(\Gamma^{*}\right)\left(\right.$ resp. $\left.\mathcal{F} \ell^{\infty, 1 ; b}\left(\Gamma^{*}\right) \times \mathcal{F}^{2,1 ; b}\left(\Gamma^{*}\right)\right)$ for those $v, w, \lambda$ and $k$ as implied by the statement of the proposition. Hence, we obtain

$$
\begin{aligned}
R(\lambda, k, 0,0)\left(1-\left(\begin{array}{cc}
v & 0 \\
0 & w
\end{array}\right) R(\lambda, k, 0,0)\right)^{-1} & \\
& =\left(D(\lambda, k, 0,0)+\left(\begin{array}{cc}
v & 0 \\
0 & w
\end{array}\right)\right)^{-1}=R(\lambda, k, v, w) .
\end{aligned}
$$

Therefore, by theorem B.1.7 $R(\lambda, k, v, w)$ maps $\mathcal{F} \ell^{\infty, 1}\left(\Gamma^{*}\right) \times \mathcal{F} \ell^{2,1}\left(\Gamma^{*}\right)$ boundedly into $\mathcal{F} \ell^{1}\left(\Gamma^{*}\right) \times \mathcal{F} \ell^{2,1}\left(\Gamma^{*}\right)$. Furthermore, by theorem B.1.9 and proposition 2.2.4 $R(\lambda, k, v, w)$ maps $\mathcal{F} \ell^{\infty, 1 ; b}\left(\Gamma^{*}\right) \times \mathcal{F} \ell^{2,1 ; \sqrt{b}}\left(\Gamma^{*}\right)$ compactly into the space $\mathcal{F} \ell^{1}\left(\Gamma^{*}\right) \times \mathcal{F} \ell^{2,1 ; \sqrt{b}}\left(\Gamma^{*}\right)$. This restricts/embeds to a compact mapping on $\mathcal{F} \ell^{2,1 ; \sqrt{b}}\left(\Gamma^{*}\right) \times \mathcal{F} \ell^{2,1 ; \sqrt{b}}\left(\Gamma^{*}\right)$. Since this space has a Schauder basis, the remaining statements follow as in proposition 3.3 .21 .

Theorem B.1.13. For all $w_{0} \in \mathcal{F} \ell^{1}\left(\Gamma^{*}\right)$ of sufficiently small norm, and all $v_{0} \in$ $\mathcal{F} \ell^{\infty, 1}\left(\Gamma^{*}\right)$ there is a nondecreasing, divergent Karamata sequence $b$ with $v_{0} \in$ $\mathcal{F} \ell^{\infty, 1 ; b}\left(\Gamma^{*}\right)$, and an open neighbourhood of both $\left(v_{0}, w_{0}\right)$ and $(0,0)$ in the function space $\mathcal{F} \ell^{\infty, 1 ; b}\left(\Gamma^{*}\right) \times \mathcal{F} \ell^{1}\left(\Gamma^{*}\right)$ which is invariant under translation operators such that for all $(k, v, w) \in \mathbb{C}^{2} \times U$ there is a discrete set $S(k, v, w) \subseteq \mathbb{C}$ of finite-order singularities of the resolvent. In particular, the map

$$
\begin{aligned}
\{(v, w, k, \lambda) & \left.\in U \times \mathbb{C}^{2} \times \mathbb{C}: \lambda \notin S(k, u)\right\} \\
& \mapsto \mathcal{K}\left(\mathcal{F} \ell^{2,1 ; \sqrt{b}}\left(\Gamma^{*}\right) \times \mathcal{F} \ell^{2,1 ; \sqrt{b}}\left(\Gamma^{*}\right), \mathcal{F} \ell^{2,1 ; \sqrt{b}}\left(\Gamma^{*}\right) \times \mathcal{F} \ell^{2,1 ; \sqrt{b}}\left(\Gamma^{*}\right)\right) \\
(v, w, k, \lambda) & \mapsto R(\lambda, k, v, w)
\end{aligned}
$$

is defined and continuous with respect to the weak topology on $U$ and the usual norm topologies otherwise. Continuity is locally uniform in $k$.

Proof. The proof is analogous to the proof of theorem 3.3.24. except that we use $\mathcal{F} \ell^{2,1 ; \sqrt{b}}\left(\Gamma^{*}\right)$ instead of $L^{2}(F)$. Proposition B.1.12 yields an open neighbourhood $U$ of potential pairs and, for each bounded $V \subseteq \mathbb{C}^{2}$, compact resolvents $R\left(\lambda_{0}, k, v, w\right)$ with a suitable $\lambda_{0} \in(-\infty,-1] i, k \in \bar{V}$ and $(v, w) \in U$, so that by the Riesz-Schauder theorem 2.2.7, the set

$$
S(k, v, w):=\left\{\lambda \in \mathbb{C} \backslash\left\{\lambda_{0}\right\}:\left(\lambda_{0}-\lambda\right)^{-1} \in \operatorname{Spec} R\left(\lambda_{0}, k, v, w\right)\right\}
$$

is discrete. For $\lambda \in \mathbb{C} \backslash S(k, v, w), \lambda \neq \lambda_{0}$, the operator

$$
\left(\lambda_{0}-\lambda\right)^{-1}-R\left(\lambda_{0}, k, v, w\right)
$$

is boundedly invertible. This yields

$$
\left(\lambda_{0}-\lambda\right)^{-1} R\left(\lambda_{0}, k, v, w\right)\left(\left(\lambda-\lambda_{0}\right)^{-1}-R\left(\lambda_{0}, k, v, w\right)\right)^{-1}=R(\lambda, k, v, w) .
$$

The remainder of the theorem (extension from $V$ to $\mathbb{C}^{2}$ and the continuity statements) follow as in theorem 3.3 .24 . 


\section{B.2 Fermi curves of Dirac operators}

\section{B.2.1 Bloch varieties and Fermi curves}

With theorem B.1.13, we are now in a position which enables us to execute basically the same programme as in section 4.1. That is, we can define the Bloch variety of the Dirac operator

$$
B_{D}(v, w):=\left\{(k, \lambda) \in \mathbb{C}^{2} \times \mathbb{C}: \text { There is a } \psi \neq 0 \text { such that } D(k, v, w) \psi=\lambda \psi\right\}
$$

and the Fermi curve of the Dirac operator

$$
F_{D}(v, w):=\left\{k \in \mathbb{C}^{2}:(k, 0) \in B_{D}(v, w)\right\} .
$$

Again, these definitions must be viewed as tentative, as we need a result similar to theorem 4.1.3 in order to establish a sound analytical footing. Before we do so, let us first expose the similarities of, and differences between Schrödinger and Dirac Fermi curves. First off, we have, as in the Schrödinger case,

$$
B_{D}(v, w)=\coprod_{\lambda \in \mathbb{C}} F_{D}(v-\lambda, w-\lambda)
$$

so the restriction to $\lambda=0$ in the Fermi curve has, per se, the same effect as the restriction to any other constant value for $\lambda$. It has to be noted, however, that in subsection B.1.3 we developed the general Dirac theory for potentials $w$ with a certain bound on the norm, which will not be satisfied for all $\lambda$ in $w-\lambda$. Luckily, in case of Fermi curves, we can prove the following scaling behaviour.

Proposition B.2.1. Let $v, w$ be potentials from vector spaces for which the Dirac equation (B.1.5 makes sense, and let $c \in \mathbb{C}$ with $c \neq 0$. Then $F_{D}(v, w)=$ $F_{D}\left(c^{-1} v, c w\right)$.

Proof. We have $k \in F_{D}(v, w)$ if and only if there is a nonzero $\psi:=\left(\psi_{1}, \psi_{2}\right)^{t}$ such that $D(k, v, w) \psi=0$. A simple calculation shows that this is the case if and only if $D(k, \widetilde{v}, \widetilde{w}) \widetilde{\psi}=0$, where $\widetilde{v}=c^{-1} v, \widetilde{w}=c w$ and $\widetilde{\psi}=\left(\widetilde{\psi}_{1}, \widetilde{\psi}_{2}\right)^{t}$ with $\widetilde{\psi}_{1}=\psi_{1}$ and $\widetilde{\psi}_{2}=c^{-1} \psi_{2}$.

This proposition allows us to identify Fermi curves for arbitrary potentials $v \in \mathcal{F} \ell^{\infty, 1}\left(\Gamma^{*}\right)$ and $w \in \mathcal{F} \ell^{1}\left(\Gamma^{*}\right)$ with Fermi curves of potentials within the domain of validity of theorem B.1.13.

Understandably, the Schrödinger Fermi curve, dependent on only one potential, has no such scaling behaviour. On the other hand, in proposition 4.5.8 we proved the symmetry $F(u)=-F(u)$, which is not shared with the Dirac Fermi curves. The same methods used to prove this proposition would derive $F_{D}(v, w)=-F_{D}(w, v)$, but since we take our potentials from different spaces, this merely shows once more that whether we take $v$ or $w$ to be the potential with Schrödinger characteristics is of no consequence. Rather, we now show the connexion between the Schrödinger and the Dirac theory in the guise of the following identity.

Theorem B.2.2. Let $u \in \mathcal{F} \ell^{\infty, 1}\left(\Gamma^{*}\right)$. Then we have the identity of Schrödinger and Dirac Fermi curves

$$
F(u)=F_{D}(u /(4 c), c)
$$

for all $c \in \mathbb{C}, c \neq 0$. 
Proof. Let $k \in F(u)$. Then there exists some non-zero solution of the Schrödinger equation $\psi_{2}$, whose second derivatives are in $\mathcal{F} \ell^{\infty, 1}\left(\Gamma^{*}\right)$ :

$$
\left(-\Delta_{k}+u\right) \psi_{2}=0 .
$$

It then follows from a calculation similar to that in the proof of proposition 3.2 .12 that the function $\psi_{1}$ defined by the equation

$$
\psi_{1}=-\frac{1}{c} \partial_{k} \psi_{2}
$$

is in $\mathcal{F} \ell^{2,1}\left(\Gamma^{*}\right)$. Now, solving $(\mathrm{B} .2 .4)$ for $-\partial_{k} \psi_{2}$ and dividing $(\mathrm{B} .2 .3$ by $4 c$ yields, using lemma 4.2 .2 , the system of equations

$$
\begin{aligned}
\bar{\partial}_{k} \psi_{1}+\frac{u}{4 c} \psi_{2} & =0, \\
c \psi_{1}+\partial_{k} \psi_{2} & =0 .
\end{aligned}
$$

Therefore, the tuple $\left(\psi_{1}, \psi_{2}\right)^{t}$ is in the kernel of $D(0, k, u /(4 c), c)$, and thus $k \in$ $F_{D}(u /(4 c), c)$.

If, on the other hand, $k \in F_{D}(u /(4 c), c)$, there is a $\psi_{1} \in \mathcal{F} \ell^{2,1}\left(\Gamma^{*}\right)$ whose first derivatives are in $\mathcal{F} \ell^{\infty, 1}\left(\Gamma^{*}\right)$ and a $\psi_{2} \in \mathcal{F} \ell^{1}\left(\Gamma^{*}\right)$ not both of which are zero such that the system $\sqrt{B .2 .5}$ is fulfilled. From the second equation, we can infer (B.2.4). This shows that the second derivatives of $\psi_{2}$ are in $\mathcal{F} l^{\infty, 1}\left(\Gamma^{*}\right)$. By plugging (B.2.4) into the first equation, we arrive at (B.2.3) with the help of lemma 4.2 .2 . Therefore, $k \in F(u)$.

In total, this shows that $F(u)=F_{D}(u /(4 c), c)$.

Note that it might seem that the previous theorem can be generalised by replacing $c$ with a nonvanishing holomorphic function $w$ because the multiplication with a holomorphic function commutes with $\bar{\partial}_{k}$. However, the condition $w \in \mathcal{F} \ell^{1}\left(\Gamma^{*}\right)$ implies that such a $w$ would be constant by Liouville's theorem for entire elliptic functions.

In any case, we see that the set of our Dirac Fermi curves is strictly larger than our set of Schrödinger Fermi curves. We have already seen with proposition B.2.1 that this provides us with a wider array of useful transformation behaviours. As already mentioned at the end of subsection B.1.1. we can use the freedom of having different boundary conditions for both $\partial$ and $\bar{\partial}$ in an intermediate step to elicit the following translation behaviour.

Proposition B.2.6. For suitable potentials $v, w$ we have, at least formally,

$$
F_{D}(v, w)=F_{D}\left(\psi_{-\kappa} v, \psi_{\kappa} w\right)+k_{\kappa}^{-}
$$

for all $\kappa \in \Gamma^{*}$.

Proof. Let $k \in F_{D}(v, w)$. Then, there exist $\psi_{1}, \psi_{2}$ such that

$$
v \psi_{1}+\partial_{k} \psi_{2}=0, \quad-\bar{\partial}_{k} \psi_{1}+w \psi_{2}=0 .
$$

Using proposition 4.2.4 and due to $k_{-\kappa}^{ \pm}=-k_{\kappa}^{ \pm}$, we see that this is equivalent to

$$
\psi_{-\kappa} v \psi_{\kappa} \psi_{1}+\partial_{k-k_{\kappa}^{-}} \psi_{2}=0, \quad-\bar{\partial}_{k-k_{\kappa}^{+}} \psi_{1}+\psi_{-\kappa} \psi_{\kappa} w \psi_{2}=0,
$$


which is, due to $k_{\kappa}^{-}-k_{\kappa}^{+}=\kappa$, in turn equivalent to

$$
\psi_{-\kappa} v\left(\psi_{\kappa} \psi_{1}\right)+\partial_{k-k_{\kappa}^{-}} \psi_{2}=0, \quad \psi_{-\kappa}\left(-\bar{\partial}_{k-k_{\kappa}^{-}}\left(\psi_{\kappa} \psi_{1}\right)+\psi_{\kappa} w \psi_{2}\right)=0 .
$$

Hence $k \in F_{D}(v, w)$ if and only if $k \in F_{D}\left(\psi_{-\kappa} v, \psi_{\kappa} w\right)+k_{\kappa}^{-}$. An eigenfunction in the latter case is given by $\psi_{\kappa} \psi_{1}$ and $\psi_{2}$.

Since Dirac Fermi curves are a generalisation of Schrödinger Fermi curves, it makes sense to prove a generalisation of theorem 4.1.3. That generalisation will not have quite the same quality as this theorem, because we are no longer in a Hilbert space setting. Nevertheless, we obtain a sound local analytic description of Dirac Bloch varieties.

Theorem B.2.7. Let $w_{0} \in \mathcal{F} \ell^{1}\left(\Gamma^{*}\right)$ have norm that is sufficiently small, $v_{0} \in$ $\mathcal{F} \ell^{\infty, 1}\left(\Gamma^{*}\right), k_{0} \in \mathbb{C}^{2}$ and $\lambda_{0} \in \mathbb{C}$. Furthermore, let $b$ be a nondecreasing, divergent Karamata sequence $b$ with $v_{0} \in \mathcal{F} \ell^{\infty, 1 ; b}\left(\Gamma^{*}\right)$ and assume there is a nonzero $\psi \in \mathcal{F} \ell^{1}\left(\Gamma^{*}\right) \times \mathcal{F} \ell^{2,1 ; \sqrt{b}}\left(\Gamma^{*}\right)$ with $D\left(k_{0}, v_{0}, w_{0}\right) \psi=\lambda_{0} \psi$. Then there are open neighbourhoods $U, V$ and $W$ of $\left(v_{0}, w_{0}\right)\left(\right.$ in $\left.\mathcal{F} \ell^{\infty, 1 ; b}\left(\Gamma^{*}\right) \times \mathcal{F} \ell^{2,1 ; \sqrt{b}}\left(\Gamma^{*}\right)\right)$, $k_{0}$ and $\lambda_{0}$, respectively, such that for all $(v, w, k, \lambda) \in U \times V \times W$ there is a finite-dimensional subspace $\Sigma(k, v, w)$ of $\mathcal{F} \ell^{2,1 ; \sqrt{b}}\left(\Gamma^{*}\right) \times \mathcal{F} \ell^{2,1 ; \sqrt{b}}\left(\Gamma^{*}\right)$ independent of $\lambda$ which is invariant under the Dirac operator $D(k, v, w)$, such that the intersection of the graph of the map $(v, w) \mapsto B_{D}(v, w)$ with $U \times V \times W$ is the zero locus of the determinant

$$
\left.(v, w, k, \lambda) \mapsto \operatorname{det} D(k, v, w)\right|_{\Sigma(k, v, w)} .
$$

This map is holomorphic in $\lambda$ and $k$, and continuous in $u$, with the weak topology on $U$ and the usual topologies elsewhere.

Proof. By theorem B.1.13, $\lambda_{0}$ is an isolated pole of the resolvent $R\left(\lambda, k_{0}, v_{0}, w_{0}\right)$. By the reverse Riesz-Schauder theorem 2.2.8, the operator

$$
P\left(k_{0}, v_{0}, w_{0}\right):=\frac{1}{2 \pi i} \oint_{\lambda_{0}} R\left(\lambda, k_{0}, v_{0}, w_{0}\right) \mathrm{d} \lambda
$$

is a $D\left(k_{0}, v_{0}, w_{0}\right)$-invariant projector. Since the resolvent is compact, the projector $P\left(k_{0}, v_{0}, w_{0}\right)$ has finite rank by proposition 2.2.5 Hence, the image of this projector $\Sigma\left(k_{0}, v_{0}, w_{0}\right)$ is finite-dimensional. Let $f_{1}, \ldots, f_{m}$ be a basis of $\Sigma\left(k_{0}, v_{0}, w_{0}\right)$. At this point in the proof of theorem 4.1.3. we considered the Hermitian adjoint of the projector. But alas, $\mathcal{F} \ell^{2,1 ; \sqrt{b}}\left(\Gamma^{*}\right)$ is not a Hilbert space. Therefore, let $f_{1}^{*}, \ldots, f_{m}^{*}$ be the dual basis to $f_{1}, \ldots, f_{m}$ and define a partial operator on the dual space $\left(\mathcal{F} \ell^{2,1 ; \sqrt{b}}\left(\Gamma^{*}\right) \times \mathcal{F} \ell^{2,1 ; \sqrt{b}}\left(\Gamma^{*}\right)\right)^{*}$ by

$$
\left(P^{*}\left(k_{0}, v_{0}, w_{0}\right) f_{i}^{*}\right)\left(f_{j}\right):=f_{i}^{*}\left(P\left(k_{0}, v_{0}, w_{0}\right) f_{j}\right)
$$

for all $i, j=1, \ldots, m$. Hence, $P^{*}\left(k_{0}, v_{0}, w_{0}\right)$ may be thought of as a finite-rank projector to the space generated by $f_{1}^{*}, \ldots, f_{m}^{*}$ (which is, of course, identical to $\Sigma\left(k_{0}, v_{0}, w_{0}\right)$ due to finite-dimensionality). The matrix defined by the matrix elements

$$
\left(P^{*}\left(k_{0}, v_{0}, w_{0}\right) f_{i}^{*}\right)\left(P\left(k_{0}, v_{0}, w_{0}\right) f_{j}\right)
$$


for $i, j=1, \ldots, m$ is simply the unity matrix and thus has nonzero determinant. Due to continuity, there are small open neighbourhoods $U, V$ and $W:=$ $B_{c}\left(\lambda_{0}\right)$ of $\left(v_{0}, w_{0}\right), k_{0}$ and for an appropriate $c>0$, respectively, such that for all $(v, w) \in U, k \in V$ the operators

$$
P(k, v, w):=\oint_{\left|\lambda-\lambda_{0}\right|<c} R(\lambda, k, v, w) \mathrm{d} \lambda
$$

are $D(k, v, w)$-invariant projectors of constant rank to finite-dimensional subspaces $\Sigma(k, v, w)$, and, with operators $P^{*}(k, v, w)$ as above, the matrices defined by the matrix elements

$$
\left(P^{*}(k, v, w) f_{i}^{*}\right)\left(P(k, v, w) f_{j}\right),
$$

$i, j=1, \ldots, m$, have nonzero determinant. Due to the $\Sigma(k, v, w)$-invariance of the Dirac operator, the matrix elements $A_{i j}(k, v, w)$ can be defined by

$$
(\lambda-D(k, v, w)) P(k, v, w) f_{i}=\sum_{j=1}^{m} A_{i j}(k, v, w) P(k, v, w) f_{j} .
$$

The matrix $A(k, v, w)$ defined by these matrix elements is singular if and only if $(v, w, k, \lambda)$ lies in the graph of $(v, w) \mapsto B_{D}(v, w)$. Now,

$$
\begin{aligned}
\left(P^{*}(k, v, w) f_{l}^{*}\right)\left((\lambda-D(k, v, w)) P(k, v, w) f_{i}\right) & \\
= & \sum_{j=1}^{m} A_{i j}(k, v, w)\left(P^{*}(k, v, w) f_{l}^{*}\right)\left(P(k, v, w) f_{j}\right)
\end{aligned}
$$

for all $i, l=1, \ldots, m$, proving the theorem just as in the proof of theorem 4.1 .3

\section{B.2.2 Free and asymptotically free Dirac Fermi curves}

In sections 4.2 and 4.3 , we explicitly calculated the free Schrödinger Fermi curve $F(0)$ and also proved the asymptotic freeness of general Schrödinger Fermi curves outside the handles. We shall now generalise these results to Fermi curves of Dirac operators. As we shall see, the first-order nature of the Dirac operator, in particular the translation behaviour proved in proposition B.2.6 greatly simplify this task in comparison with the Schrödinger case.

Proposition B.2.8. Recall the definition of $\mathcal{R}$ in 4.2 .6 . The free Fermi curve is given by $F_{D}(0,0)=\mathcal{R}+\Gamma^{*}$. Furthermore, $\mathcal{R}$ is a system of representatives for the quotient $F_{D}(0,0) / \Gamma^{*}$, provided that the pairs of distinct points $\left(k_{\kappa}^{-}, k_{\kappa}^{+}\right)$ are identified to double points for all $\kappa \in \Gamma^{*}, \kappa \neq 0$.

Proof. Let $k \in F_{D}(0,0)$. Then there are functions $\psi_{1}, \psi_{2}$ not both of which are zero such that

$$
\bar{\partial}_{k} \psi_{1}=0, \quad \partial_{k} \psi_{2}=0 .
$$

A Fourier transform yields the equations

$$
\begin{aligned}
& \pi i\left(\left(\kappa_{1}+k_{1}\right)-i\left(\kappa_{2}+k_{2}\right)\right) \widehat{\psi}_{1}(\kappa)=0, \\
& \pi i\left(\left(\kappa_{1}+k_{1}\right)+i\left(\kappa_{2}+k_{2}\right)\right) \widehat{\psi}_{2}(\kappa)=0 .
\end{aligned}
$$


Since not both $\psi_{1}, \psi_{2}$ are zero, there is a $\kappa \in \Gamma^{*}$ such that $(4.2 .9)$ holds. The remainder of the proof is now identical to the remainder of the proof of theorem 4.2.5.

This proposition shows that $F_{D}(0,0)=F(0)$ (see figure 4.2). We already know from theorem B.2.2 that $F(0)=F_{D}(0, c)$ for all $c \in \mathbb{C}, c \neq 0$. Indeed, it is possible to show that $F(0)=F_{D}(v, 0)=F_{D}(0, w)$ for all $v, w \in \mathcal{F} \ell^{\infty, 1}\left(\Gamma^{*}\right)$.

Recall that the free Fermi curve $F_{D}(0,0)=F(0)$ is invariant under translations by lattice vectors from the lattice $\Gamma_{\mathbb{C}}^{*}$. Furthermore, general Fermi curves $F_{D}(v, w)$ are invariant under translations by lattice vectors from the lattice $\Gamma^{*}$ : this can be shown exactly as in lemma 4.3.1.

In theorems 3.3.24 and B.1.13 we proved strong continuity statements, namely that the resolvent is continuous with respect to the potentials even with only the weak topology on the (strongly bounded) set of potentials under consideration. Essentially, this was an additional upshot from the compactness of the resolvent. In terms of Dirac Fermi curves, it implies the following behaviour.

Proposition B.2.10. Let $b$ be a nondecreasing, divergent Karamata sequence, $\left(v_{n}\right)_{n \in \mathbb{N}}$ a sequence of $\mathcal{F} \ell^{\infty, 1 ; b}\left(\Gamma^{*}\right)$-potentials in $B_{C_{1}}^{N}$ and $\left(w_{n}\right)_{n \in \mathbb{N}}$ a sequence of $\mathcal{F} \ell^{1}\left(\Gamma^{*}\right)$-potentials whose norm does not exceed $C_{2}$, where $N, C_{1}$, and $C_{2}$ are constants compatible with the prerequisites of proposition B.1.12. Assume further that the sequence $\left(v_{n}, w_{n}\right)_{n \in \mathbb{N}}$ converges weakly to the zero potentials $(0,0)$. Then for any bounded $V \subseteq \mathbb{C}^{2}$, the sequence $\left(V \cap F_{D}\left(v_{n}, w_{n}\right)\right)_{n \in \mathbb{N}}$ converges to the respective part of the free Fermi curve, namely $V \cap F_{D}(0,0)$ with respect to the Hausdorff distance (see section 4.3).

Proof. With our choices for $N, C_{1}$, and $C_{2}, F_{D}\left(v_{n}, w_{n}\right)$ converges pointwise to $F_{D}(0,0)$ by theorem B.1.13. Since the resolvent $R(\lambda, k, v, w)$ is locally uniformly continuous in $k$, the proposition follows because $V$ is bounded.

Of course, an analogous convergence property holds for Schrödinger Fermi curves, due to theorem 3.3.24. The Dirac version, however, has the advantage of being quite directly usable to derive the asymptotic freeness of Dirac Fermi curves in analogy to corollary 4.3.9. Just as in the Schrödinger case, the above continuity statement fails near the double points of the free Fermi curve when one passes from $F_{D}(v, w)$ to $F_{D}(v, w) / \Gamma^{*}$. Therefore, we defined the handles in definition 4.3.2 and, in order to investigate the behaviour of Fermi curves outside the handles, we made a size estimate in lemma 4.3 .3 based on the fact that the eigenvalues of the Schrödinger operator become sparser for large $k$. The Dirac operator, being a first-order differential operator, has no such sparsity behaviour, nor is it needed. Instead, we can use a combination of proposition B.2.6 and proposition B.2.10.

For $\epsilon, \delta>0$, define

$U_{\epsilon, \delta}^{ \pm}:=\left\{k \in \mathbb{C}^{2}:\left\|i k_{1} \pm k_{2}\right\|<\epsilon,\|k\|>\delta^{-1},\left\|k-k_{\kappa}^{ \pm}\right\|>\epsilon\right.$ for all $\left.\kappa \in \Gamma^{*}, \kappa \neq 0\right\}$.

For small $\epsilon$ and $\delta$ (where the latter may depend on the former), these sets contain points near the free Fermi curve $F_{D}(0,0)$ sufficiently far removed from the origin, and away from the double points. If $\epsilon$ is small enough, we may take $U_{\epsilon, \delta}^{ \pm}$to be a subset of $\mathbb{C}^{2} / \Gamma^{*}$ by choosing an appropriate fundamental domain (remember that this fundamental domain is not bounded). For such $\epsilon, v \in \mathcal{F} \ell^{\infty, 1}\left(\Gamma^{*}\right)$, and 
$w \in \mathcal{F} \ell^{1}\left(\Gamma^{*}\right)$ with sufficiently small norm, we define the parts of $F_{D}(v, w) / \Gamma^{*}$ meeting $U_{\epsilon, \delta}^{ \pm}$:

$$
V_{\epsilon, \delta}^{ \pm}(v, w):=U_{\epsilon, \delta}^{ \pm} \cap F_{D}(v, w) / \Gamma^{*}
$$

(here, an appropriate choice of a Lorentz-Karamata space for $v$ is implicit). Recall the definition of $\Gamma_{\delta}^{*}$ from (4.3.6), as well as the definitions of $F_{\mathbb{C}}$ and $F_{\mathbb{C}}^{\epsilon}$. We can now state the following decomposition theorem for our Dirac Fermi curves $F_{D}(v, w) / \Gamma^{*}$ :

Theorem B.2.11. Let $v \in \mathcal{F} \ell^{\infty, 1}\left(\Gamma^{*}\right)$, and let $w \in \mathcal{F} \ell^{1}\left(\Gamma^{*}\right)$ have sufficiently small norm. Then for all sufficiently small $\epsilon>0$ there is a $\delta>0$ and a compact set

$$
K \subseteq\left\{k \in F_{D}(v, w) / \Gamma^{*} \subseteq \mathbb{C}^{2} / \Gamma^{*}:\|k\| \leq \delta^{-1}\right\}
$$

along with a set of handles $\left(H_{\kappa}\right)_{\kappa \in \Gamma_{\delta}^{*}}$ of thickness not exceeding $\epsilon$ such that we have the following disjoint decomposition of $F_{D}(v, w) / \Gamma^{*}$ :

$$
F_{D}(v, w) / \Gamma^{*}=V_{\epsilon, \delta}^{+}(v, w) \cup V_{\epsilon, \delta}^{-}(v, w) \cup K \cup \bigcup_{\kappa \in \Gamma_{\delta}^{*}}\left(H_{\kappa} \cap F_{D}(v, w)\right) / \Gamma^{*} .
$$

Proof. By theorem A.1.3 we may assume $v \in \mathcal{F} \ell^{\infty, 1 ; b}\left(\Gamma^{*}\right)$ for a suitable nondecreasing, divergent Karamata sequence $b$. Then $\psi_{-\kappa} v \in \mathcal{F} \ell^{\infty, 1 ; b}\left(\Gamma^{*}\right)$ as well, for all $\kappa \in \Gamma^{*}$. Now, the sequence $\left(\psi_{-\kappa} v, \psi_{\kappa} w\right)_{\kappa \in \Gamma^{*}}$ converges weakly to the zero potentials $(0,0)$ as $\|\kappa\| \rightarrow \infty$ because the Fourier coefficients of $v$ and $w$ vanish in this limit. This sequence is obviously bounded, so there is a $\delta>0$ such that for all $\kappa \in \Gamma_{\delta}^{*}$, the Hausdorff distance from $F_{D}\left(\psi_{-\kappa} v, \psi_{\kappa} w\right) \cap F_{\mathbb{C}}^{\epsilon}$ to $F_{D}(0,0) \cap F_{\mathbb{C}}^{\epsilon}$ is smaller than $\epsilon$ by proposition B.2.10. Due to proposition B.2.6, this is also true for all $k \in F_{D}(v, w)$ of the form $k=k^{\prime}+k_{\kappa}^{+}$with $k^{\prime} \in F_{\mathbb{C}}^{\epsilon}$ and $\kappa \in \Gamma_{\delta}^{*}$, i.e. $k+\Gamma^{*} \in V_{\epsilon, \delta}^{ \pm}(v, w)$. By construction, the remaining $k+\Gamma^{*}$ are either in $K$ or in $H_{\kappa} / \Gamma^{*}$ for a suitable $\kappa \in \Gamma^{*}$.

This theorem directly yields the result analogous to corollary 4.3 .9 for Dirac Fermi curves (and reproduces the Schrödinger result due to theorem B.2.2).

\section{B.3 Asymptotic analysis of Dirac Fermi curves}

Now that we know the asymptotic behaviour of Dirac Fermi curves outside the handles, we shall now investigate the handles, just as we did with the Schrödinger Fermi curve in section 4.5. In the end, we will fail to reproduce the results from the Schrödinger case, which is all the more irksome, because initially, this task will, in places, turn out to be much simpler than in the Schrödinger case. For one, we can use proposition B.2.6 to move the double point in question into the origin. This means that instead of a family of spaces $\left\{K_{\nu}\right\}_{\nu \in \Gamma^{*}}$, we need only consider a single space $K$ corresponding to the zeroth Fourier mode (note that since the Dirac operator is a $2 \times 2$-matrix operator, $K$ is still a two-dimensional space). Furthermore, since the Dirac operator is only of first order, some of the arithmetic in section 4.5 is simplified. Finally, we do not need to take special care of the constant parts of the potentials $v$ and $w$.

However, our deliberate breaking of the symmetry between $v$ and $w$ by choosing different spaces for the potentials does not reconcile with the Dirac analogon of the nonlinear perturbation of the Fourier transform which we developed for the Schrödinger operator. The details are laid out in what follows. 


\section{B.3.1 A nonlinear perturbation of the Fourier transform}

Let $E$ be a Banach space some rearrangement-invariant Banach function space on $\Gamma^{*}$. Furthermore, let $K$ denote the subspace of $E$ corresponding to $\kappa=0$ (that is, the image of the operator of multiplication with $\delta_{\kappa 0}$ ), and let $K^{\perp}$ denote the canonical complement of $K$, such that $E=K \oplus K^{\perp}$ (cf. definition 4.5.1). This carries over to the Fourier space of $E$. Since $\psi_{0}=1, K$ simply corresponds to the constant functions in this case. For example, if $E=\mathcal{F} \ell^{2,1 ; b}\left(\Gamma^{*}\right) \times \mathcal{F} \ell^{1}\left(\Gamma^{*}\right)$, then $K \cong \mathbb{C} \times \mathbb{C}$. The projection $\pi_{K}$ onto $K$ is given by the zeroth Fourier coefficient. In cases where the identification 2.1.15 from proposition 2.1.14 makes sense, this projection is a simple integration:

$$
\pi_{K}: E \rightarrow K, \quad f \mapsto \int_{F} f \mathrm{~d} \mu .
$$

Otherwise, one must rely on the abstract rendition 2.1.13 of the Fourier transform. We shall always denote these subspaces by $K$ and $K^{\perp}$, respectively, regardless of the underlying space. The relevance of $K$ here is that it is the kernel of the free Dirac operator $D(0,0,0)$ :

Lemma B.3.1. The kernel of $D(0,0,0)$ is $K$.

Proof. The equation $D(0,0,0) \psi=0$ implies

$$
-\bar{\partial} \psi_{1}=0, \quad \partial \psi_{2}=0 .
$$

A Fourier transform yields

$$
-\pi\left(i \kappa_{1}+\kappa_{2}\right) \widehat{\psi}_{1}(\kappa)=0, \quad \pi\left(i \kappa_{1}-\kappa_{2}\right) \widehat{\psi}_{2}(\kappa)=0
$$

by proposition 2.1.6. Since $\kappa_{1}, \kappa_{2} \in \mathbb{R}$ for all $\kappa \in \Gamma^{*}$, these equations can only

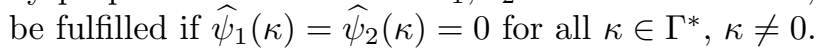

This lemma shows that $K$ is the singular support of the resolvent $R(0,0,0,0)$ (as is expected from proposition B.2.8). In order to gain an explicit local description of the Fermi curve, we shall determine the singular support of $R(0, k, v, w)$ for $k$ in a small open neighbourhood of zero and $v, w$ from small weakly open neighbourhoods of the zero potentials.

Warning. As in section 4.5 , we shall want to investigate $K \oplus K^{\perp}$-decompositions of operators, yielding $2 \times 2$-matrix operators, as suggested by definition 4.5.3. This rendition of operators in $2 \times 2$-matrix form has nothing to do with the natural $2 \times 2$-matrix form which arises when the underlying spaces are product spaces, such as the Dirac operator introduced in section B.1. Indeed, if the underlying spaces are product spaces, the entries of the $2 \times 2$ operator matrices with respect to $K \oplus K^{\perp}$ will be $2 \times 2$-matrices with respect to the product.

Proposition B.3.2. There is an open neighbourhood $V$ of $0 \in \mathbb{C}^{2}$ which depends only on $\Gamma^{*}$, and on which the free resolvent $k \mapsto R(0, k, 0,0)$ has the $K \oplus K^{\perp}$ decomposition

$$
R(0, k, 0,0)=\left(\begin{array}{cc}
S(k) & 0 \\
0 & R_{r}(k)
\end{array}\right)
$$


where $R_{r}$ is holomorphic on $V$ (that is, the singular support of $R_{r}$ is empty) ${ }^{2}$ and $S(k)$ is given by

$$
S(k)=\left(\begin{array}{cc}
0 & \pi i k_{1}+\pi k_{2} \\
-\pi i k_{1}+\pi k_{2} & 0
\end{array}\right)^{-1}
$$

with respect to the canonical basis of $K \cong \mathbb{C} \times \mathbb{C}$.

Proof. The proof is similar to the proof of proposition 4.5.4 Let $k \in \mathbb{C}^{2}$ be a pole of $k \mapsto R(0, k, 0,0)$. Then by theorem B.2.8, there is a $\kappa \in \Gamma^{*}$ such that one of the following equations holds:

$$
k_{2}+\kappa_{2}=i\left(k_{1}+\kappa_{1}\right), \quad k_{2}+\kappa_{2}=-i\left(k_{1}+\kappa_{1}\right) .
$$

If the norm of $k$ is sufficiently small (as in subsection 4.5.1, smaller than half the minimum distance of lattice vectors in $\Gamma_{\mathbb{C}}^{*}$ ), this can only happen if $\kappa=$ 0 because $\kappa_{1}, \kappa_{2} \in \mathbb{R}$. All possible poles are contained in $S(k)$ in this case. Hence, $R_{r}$ is pole-free. The form of $S(k)$ follows by considering the Fourier transform of the Dirac operator at $\kappa=0$, in an analogous way to the proof of proposition 4.5.4.

This proposition shows that the entire information about the Dirac Fermi curve $F_{D}(0,0)$ near the origin is contained in the restriction of the operator $R(0, k, 0,0)$ to $K$, that is, the singular part $S(k)$. Indeed, as theorem B.2.8 tells us, this Fermi curve is determined by the equation

$$
\operatorname{det}\left(\begin{array}{cc}
0 & \pi i k_{1}+\pi k_{2} \\
-\pi i k_{1}+\pi k_{2} & 0
\end{array}\right)=0,
$$

that is, $k^{2}=0$ near the origin. From now on, we always let $V$ be as in the previous proposition. In the remainder of this section, we shall generalise this result to small weakly open neighbourhoods of the zero potentials.

Instead of proving an analogon of lemma 4.5.12 and working from there, we shall exploit the weak continuity properties of the resolvent. For this purpose, we first need three simple lemmata concerning operator inverses.

Lemma B.3.3. There is a $\lambda_{0} \in(-\infty,-1] i$ such that for all $\lambda$ with $\lambda / i \leq$ $\lambda_{0} / i$ and all $k \in V$ the operator $R(\lambda, k, 0,0) D(k, 0,0)$ maps $K^{\perp} \subseteq \mathcal{F} \ell^{1}\left(\Gamma^{*}\right) \times$ $\mathcal{F} \ell^{2,1}\left(\Gamma^{*}\right)$ boundedly into itself. Furthermore, this operator is boundedly invertible on $K^{\perp} \subseteq \mathcal{F} \ell^{1}\left(\Gamma^{*}\right) \times \mathcal{F} \ell^{2,1}\left(\Gamma^{*}\right)$. The inverse is given by

$$
\left.R_{r}(k)(\lambda-D(k, 0,0))\right|_{K^{\perp}} .
$$

Proof. The Fourier transform of $D(k, 0,0)$ is the multiplication with the matrix

$$
\left(\begin{array}{cc}
0 & \pi i\left(\kappa_{1}+k_{1}\right)+\pi\left(\kappa_{2}+k_{2}\right) \\
-\pi i\left(\kappa_{1}+k_{1}\right)+\pi\left(\kappa_{2}+k_{2}\right) & 0
\end{array}\right) .
$$

The Fourier transform of $R(\lambda, k, 0,0)$ is given by (B.1.8). For a suitable choice of $\lambda_{0}$ (see theorem B.1.7) the Fourier transform of $R(\lambda, k, 0,0) D(k, 0,0)$ is a

\footnotetext{
${ }^{2}$ Here, the subscript $r$ stands for "reduced," and is added to avoid confusion with the resolvent of the Dirac operator.
} 
simple matrix multiplication with a matrix whose entries are bounded with respect to the Fourier transform variable $\kappa$. Hence, $K^{\perp} \subseteq \mathcal{F} \ell^{1}\left(\Gamma^{*}\right) \times \mathcal{F} \ell^{2,1}\left(\Gamma^{*}\right)$ is mapped boundedly into itself. For $\kappa \neq 0$, the determinants of the matrices (B.1.8) and (B.3.4) are nonzero. Therefore, $R(\lambda, k, 0,0) D(k, 0,0)$ is boundedly invertible on $K^{\perp}$, and the inverse is given by

$$
\left(\left.R(\lambda, k, 0,0) D(k, 0,0)\right|_{K^{\perp}}\right)^{-1}=R_{r}(k)(\lambda-D(k, 0,0)) .
$$

Lemma B.3.5. Let $v_{0} \in \mathcal{F} \ell^{\infty, 1}\left(\Gamma^{*}\right)$. Then for all $\lambda / i \leq \lambda_{0} / i$, all $k \in V$, all $v \in B_{C_{1}}^{N}$ and all $w \in \mathcal{F} \ell^{1}\left(\Gamma^{*}\right)$ with $\|w\|_{\mathcal{F} 1}<C_{2}$, where the constants $\lambda_{0}, N$, $C_{1}$ and $C_{2}$ are compatible with lemma B.1.10, the operator $R_{r}(k)\left(1-\pi_{K}\right)(\lambda-$ $D(k, v, w))$ is boundedly invertible on $K^{\perp} \subseteq \mathcal{F} \ell^{1}\left(\Gamma^{*}\right) \times \mathcal{F} \ell^{2,1}\left(\Gamma^{*}\right)$.

Proof. By proposition 2.4.15 the operator

$$
\left(\begin{array}{cc}
v & 0 \\
0 & w
\end{array}\right)
$$

maps $\mathcal{F} \ell^{1}\left(\Gamma^{*}\right) \times \mathcal{F} \ell^{2,1}\left(\Gamma^{*}\right)$ boundedly into $\mathcal{F} \ell^{\infty, 1}\left(\Gamma^{*}\right) \times \mathcal{F} \ell^{2,1}\left(\Gamma^{*}\right)$. By theorem B.1.7, $R(\lambda, k, 0,0)$ maps $\mathcal{F} \ell^{\infty, 1}\left(\Gamma^{*}\right) \times \mathcal{F} \ell^{2,1}\left(\Gamma^{*}\right)$ boundedly into $\mathcal{F} \ell^{1}\left(\Gamma^{*}\right) \times$ $\mathcal{F} \ell^{2,1}\left(\Gamma^{*}\right)$. By lemma B.1.10 the operator

$$
\left(\begin{array}{cc}
v & 0 \\
0 & w
\end{array}\right) R(\lambda, k, 0,0)
$$

maps $\mathcal{F} \ell^{\infty, 1}\left(\Gamma^{*}\right) \times \mathcal{F} \ell^{2,1}\left(\Gamma^{*}\right)$ boundedly into itself, and a norm equivalent to the standard norm of this space can be found such that the norm of this operator is smaller than one. The same holds for the operator

$$
\left(1-\pi_{K}\right)\left(\begin{array}{cc}
v & 0 \\
0 & w
\end{array}\right) R(\lambda, k, 0,0)
$$

because the monotonicity property of the standard norm of the space $\ell^{\infty, 1}\left(\Gamma^{*}\right) \times$ $\ell^{2,1}\left(\Gamma^{*}\right)$ passes to the equivalent norm which we introduced in the proof of lemma B.1.10. It follows from the Neumann theorem that

$$
1-\left(1-\pi_{K}\right)\left(\begin{array}{cc}
v & 0 \\
0 & w
\end{array}\right) R(\lambda, k, 0,0)
$$

is boundedly invertible on $\mathcal{F} \ell^{\infty, 1}\left(\Gamma^{*}\right) \times \mathcal{F} \ell^{2,1}\left(\Gamma^{*}\right)$. Hence, by lemma 4.5.23, the operator

$$
1-R(\lambda, k, 0,0)\left(1-\pi_{K}\right)\left(\begin{array}{cc}
v & 0 \\
0 & w
\end{array}\right)
$$

is boundedly invertible on $\mathcal{F} \ell^{1}\left(\Gamma^{*}\right) \times \mathcal{F} \ell^{2,1}\left(\Gamma^{*}\right)$. The Fourier transform of the resolvent $R(\lambda, k, 0,0)$ shows that it leaves $K^{\perp}$ invariant. Therefore, we can apply lemma 4.5 .14 and deduce that

$$
1_{K^{\perp}}-\left.R(\lambda, k, 0,0)\left(1-\pi_{K}\right)\left(\begin{array}{cc}
v & 0 \\
0 & w
\end{array}\right)\right|_{K^{\perp}}
$$


is boundedly invertible on $K^{\perp} \subseteq \mathcal{F} \ell^{1}\left(\Gamma^{*}\right) \times \mathcal{F} \ell^{2,1}\left(\Gamma^{*}\right)$. Applying lemma B.3.3. we obtain

$$
\begin{gathered}
\left.\left(1_{K^{\perp}}-\left.R(\lambda, k, 0,0)\left(1-\pi_{K}\right)\left(\begin{array}{cc}
v & 0 \\
0 & w
\end{array}\right)\right|_{K^{\perp}}\right)^{-1} R(\lambda, k, 0,0) D(k, 0,0)\right|_{K^{\perp}} \\
=\left(R_{r}(k)\left(\left.(\lambda-D(k, 0,0))\right|_{K^{\perp}}-\left.\left(1-\pi_{K}\right)\left(\begin{array}{cc}
v & 0 \\
0 & w
\end{array}\right)\right|_{K^{\perp}}\right)^{-1}\right. \\
=\left(\left.R_{r}(k)\left(1-\pi_{K}\right)(\lambda-D(k, v, w))\right|_{K^{\perp}}\right)^{-1} .
\end{gathered}
$$

This proves the lemma.

Lemma B.3.6. Let $v_{0} \in \mathcal{F} \ell^{\infty, 1}\left(\Gamma^{*}\right)$. Then for all $v \in B_{C_{1}}^{N} \cap \mathcal{F} \ell^{\infty, 1 ; b}\left(\Gamma^{*}\right)$ and all $w \in \mathcal{F} \ell^{1}\left(\Gamma^{*}\right)$ with $\|w\|_{\mathcal{F} 1}<C_{2}$, where the constants $N, C_{1}$ and $C_{2}$ and the Karamata sequence $b$ are compatible with proposition B.1.12, the operator

$$
1-R(0, k, 0,0)\left(1-\pi_{K}\right)\left(\begin{array}{cc}
v & 0 \\
0 & w
\end{array}\right)
$$

is boundedly invertible on $\mathcal{F} \ell^{1}\left(\Gamma^{*}\right) \times \mathcal{F} \ell^{2,1 ; \sqrt{b}}\left(\Gamma^{*}\right)$ for all those $k \in V$ for which the restriction of $k \mapsto R(0, k, v, w)$ to $K^{\perp}$ does not have a pole in the sense of theorem B.1.13, that is, zero is in the resolvent set of said restriction.

Proof. By proposition B.1.12 the resolvent $R(\lambda, k, v, w)$ maps the function space $\mathcal{F} \ell^{1}\left(\Gamma^{*}\right) \times \mathcal{F} \ell^{2,1 ; \sqrt{b}}\left(\Gamma^{*}\right)$ compactly into itself for a suitable $\lambda$. This is also true of the restriction of this operator to $K^{\perp}$. If zero is in the resolvent set of the restriction of $R(0, k, v, w)$ to $K^{\perp}$, then

$$
1_{K^{\perp}}-\left.\left(1-\pi_{K}\right) \lambda R(\lambda, k, v, w)\right|_{K^{\perp}}
$$

is boundedly invertible on $K^{\perp} \subseteq \mathcal{F} \ell^{1}\left(\Gamma^{*}\right) \times \mathcal{F} \ell^{2,1 ; \sqrt{b}}\left(\Gamma^{*}\right)$. Hence, lemma B.3.5 implies

$$
\begin{gathered}
\left(1_{K^{\perp}}-\left.\left(1-\pi_{K}\right) \lambda R(\lambda, k, v, w)\right|_{K^{\perp}}\right)^{-1}\left(\left.R_{r}(k)\left(1-\pi_{K}\right)(\lambda-D(k, v, w))\right|_{K^{\perp}}\right)^{-1} \\
=\left(\left.R_{r}(k)\left(1-\pi_{K}\right)(\lambda-D(k, v, w))\right|_{K^{\perp}}-\left.R_{r}(k)\left(1-\pi_{K}\right) \lambda\right|_{K^{\perp}}\right)^{-1} \\
=\left(-R_{r}(k)\left(1-\pi_{K}\right)\left(\left.D(k, 0,0)\right|_{K^{\perp}}+\left.\left(\begin{array}{cc}
v & 0 \\
0 & w
\end{array}\right)\right|_{K^{\perp}}\right)\right)^{-1} \\
=\left(-1_{K^{\perp}}+\left.R_{r}(k)\left(1-\pi_{K}\right)\left(\begin{array}{cc}
v & 0 \\
0 & w
\end{array}\right)\right|_{K^{\perp}}\right)^{-1} .
\end{gathered}
$$

The lemma now follows from lemma 4.5.14.

We can now prove a proposition similar to proposition 4.5.15

Proposition B.3.7. Let $v_{0} \in \mathcal{F} \ell^{\infty, 1}\left(\Gamma^{*}\right)$. Then for all $v \in B_{C_{1}}^{N} \cap \mathcal{F} \ell^{\infty, 1 ; b}\left(\Gamma^{*}\right)$ and all $w \in \mathcal{F} \ell^{1}\left(\Gamma^{*}\right)$ with $\|w\|_{\mathcal{F} 1}<C_{2}$, where the constants $N, C_{1}$ and $C_{2}$ and the Karamata sequence $b$ are compatible with proposition B.1.12, the intersection of the Fermi curve $F_{D}(v, w)$ with $V$ is characterised by the poles of

$$
\begin{aligned}
& k \mapsto\left(1-R(0, k, 0,0) \pi_{K}\left(\begin{array}{cc}
v & 0 \\
0 & w
\end{array}\right)\right. \\
&\left.\cdot\left(1-R(0, k, 0,0)\left(1-\pi_{K}\right)\left(\begin{array}{cc}
v & 0 \\
0 & w
\end{array}\right)\right)^{-1}\right)^{-1}
\end{aligned}
$$


for those $k \in V$ for which $k \notin F_{D}(0,0)$.

Proof. Set

$$
U:=\left(\begin{array}{cc}
v & 0 \\
0 & w
\end{array}\right) .
$$

From now on, we shall write operators in $K \oplus K^{\perp}$ matrix from. Lemma B.3.6 ensures that the operator

$$
1-\left(\begin{array}{cc}
0 & 0 \\
0 & R_{r}(k)
\end{array}\right) U
$$

is boundedly invertible on $\mathcal{F} \ell^{1}\left(\Gamma^{*}\right) \times \mathcal{F} \ell^{2,1 ; \sqrt{b}}\left(\Gamma^{*}\right)$ for all those $k \in V$ for which the restriction of $R(0, k, v, w)$ to $K^{\perp}$ does not have a pole. Now, $R(0, k, 0,0)$ has a pole at $k \in V$ if and only if $k \in F_{D}(0,0)$. Hence we have the operator equation

$$
\begin{aligned}
& \left(1-\left(\begin{array}{cc}
0 & 0 \\
0 & R_{r}(k)
\end{array}\right) U\right)^{-1} \\
& \cdot\left(1-\left(\begin{array}{cc}
S(k) & 0 \\
0 & 0
\end{array}\right) U\left(1-\left(\begin{array}{cc}
0 & 0 \\
0 & R_{r}(k)
\end{array}\right) U\right)^{-1}\right)^{-1} R(0, k, 0,0) \\
& \quad=\left(1-\left(\begin{array}{cc}
0 & 0 \\
0 & R_{r}(k)
\end{array}\right) U-\left(\begin{array}{cc}
S(k) & 0 \\
0 & 0
\end{array}\right) U\right)^{-1} R(0, k, 0,0) \\
& \quad=(1-R(0, k, 0,0) U)^{-1} R(0, k, 0,0)=R(0, k, v, w) .
\end{aligned}
$$

This proves the claim.

With this proposition, we can now define a $2 \times 2$-matrix just as in definition 4.5.18,

Definition B.3.8. For all $k \in \mathbb{C}^{2}$, all $v \in \mathcal{F} \ell^{\infty, 1}\left(\Gamma^{*}\right)$ and all $w \in \mathcal{F} \ell^{1}\left(\Gamma^{*}\right)$ such that the operator

$$
1-\left(1-\pi_{K}\right) R(0, k, 0,0)\left(\begin{array}{cc}
v & 0 \\
0 & w
\end{array}\right)
$$

exists and is boundedly invertible on $\mathcal{F} \ell^{1}\left(\Gamma^{*}\right) \times \mathcal{F} \ell^{2,1 ; \sqrt{b}}\left(\Gamma^{*}\right.$ ) (for a suitable Karamata sequence $b)$, let $\mathcal{A}(k, v, w)$ be the restriction of the operator

$$
\left(\begin{array}{cc}
v & 0 \\
0 & w
\end{array}\right)\left(1-\left(1-\pi_{K}\right) R(0, k, 0,0)\left(\begin{array}{cc}
v & 0 \\
0 & w
\end{array}\right)\right)^{-1}
$$

to $K$, that is, a $2 \times 2$-matrix with respect to the basis generated by the two zero modes of the Fourier transform.

As of yet, we still haven't introduced the parameter $\nu$ from section 4.5 This will change in the following analogon of theorem 4.5.19. Nevertheless, the following theorem will still be conceptually slightly simpler than theorem 4.5.19 because both the weak continuity properties and the fact that the Dirac operator is a first-order (instead of a second-order) differential operator can be exploited. 
Theorem B.3.9. Let $v_{0} \in \mathcal{F} \ell^{\infty, 1}\left(\Gamma^{*}\right)$. Then there is a $\delta>0$ such that for all $\nu \in \Gamma_{\delta}^{*}, v \in B_{C_{1}}^{N} \cap \mathcal{F} \ell^{\infty, 1 ; b}\left(\Gamma^{*}\right)$ and all $w \in \mathcal{F} \ell^{1}\left(\Gamma^{*}\right)$ with $\|w\|_{\mathcal{F} 1}<C_{2}$, where the constants $N, C_{1}$ and $C_{2}$ and the Karamata sequence $b$ are compatible with proposition B.1.12 the intersection of the Fermi curve $F_{D}\left(\psi_{-\nu} v, \psi_{\nu} w\right)$ with $V$ is described by the zero locus of

$$
k \mapsto \operatorname{det}\left(\left(\begin{array}{cc}
0 & \pi i k_{1}+\pi k_{2} \\
-\pi i k_{1}+\pi k_{2} & 0
\end{array}\right)+\mathcal{A}\left(k, \psi_{-\nu} v, \psi_{\nu} w\right)\right) .
$$

For $v, w$ of sufficiently small norm, the same statement holds for all $\nu \in \Gamma^{*}$.

Proof. The $k \in F_{D}(0,0)$ part follows in a similar fashion as in the proof of theorem 4.5 .19 (consider the Fourier transform of $D(k, 0,0)$ ). Now let $k \notin F_{D}(0,0)$. The resolvent $R(0, k, 0,0)$ is pole-free on $K^{\perp}$. By theorem B.1.13, this resolvent is continuous with the weak topology on the potentials and the usual topologies elsewhere. Therefore, $k \mapsto R(0, k, v, w)$ is also pole-free on $K^{\perp}$ for $v, w$ with sufficiently small norm or for $v, w$ from a sufficiently small weakly open neighbourhood of zero. In the latter case, we arrive at the pole-free map $k \mapsto R\left(0, k, \psi_{-\nu} v, \psi_{\nu} w\right)$ on $K^{\perp}$ for all $\nu \in \Gamma_{\delta}^{*}$ for sufficiently small $\delta$. Hence, we may apply proposition 4.5.15, that is, the operator

$$
\begin{gathered}
1-R(0, k, 0,0) \pi_{K}\left(\begin{array}{cc}
\psi_{-\nu} v & 0 \\
0 & \psi_{\nu} w
\end{array}\right) \\
\cdot\left(1-R(0, k, 0,0)\left(1-\pi_{K}\right)\left(\begin{array}{cc}
\psi_{-\nu} v & 0 \\
0 & \psi_{\nu} w
\end{array}\right)\right)^{-1}
\end{gathered}
$$

has a pole at $k$ if and only if $k \in F_{D}\left(\psi_{-\nu} v, \psi_{\nu} w\right)$. Hence, by lemma 4.5.14 the Fermi curve $F_{D}\left(\psi_{-\kappa} v, \psi_{\kappa} w\right)$ is locally determined by the equation

$$
\operatorname{det}\left(1_{K}-S(k) \mathcal{A}\left(k, \psi_{-\kappa} v, \psi_{\kappa} w\right)\right)=0
$$

in this case. Multiplication with

$$
\operatorname{det}\left(\left.\pi_{K} D(0, k, 0,0)\right|_{K}\right)
$$

now implies the theorem because this determinant is nonzero if $k \notin F_{D}(0,0)$.

As in subsection 4.5.1, the form of the matrix

$$
\left(\begin{array}{cc}
0 & \pi i k_{1}+\pi k_{2} \\
-\pi i k_{1}+\pi k_{2} & 0
\end{array}\right)+\mathcal{A}\left(k, \psi_{-\nu} v, \psi_{\nu} w\right)
$$

decides the fates of the double points when potentials are switched on. Let us now derive properties for $\mathcal{A}$ as we did in subsection 4.5.1

Lemma B.3.12. For all $v_{0} \in \mathcal{F} \ell^{\infty, 1}\left(\Gamma^{*}\right)$ there is a $\delta>0$ such that the matrix $\mathcal{A}\left(k, \psi_{-\nu} v, \psi_{\nu} w\right)$ is continuously differentiable in $k$ for all $\nu \in \Gamma_{\delta}^{*}$, and we have

$$
\lim _{\|\nu\| \rightarrow \infty}\left\|\frac{\partial}{\partial k} \mathcal{A}\left(k, \psi_{-\nu} v, \psi_{\nu} w\right)\right\|=0
$$

uniformly in $k \in V$, for all $v \in \mathcal{F} \ell^{\infty, 1 ; b}\left(\Gamma^{*}\right) \cap B_{C_{1}}^{N}$ and all $w \in \mathcal{F} \ell^{1}\left(\Gamma^{*}\right)$ with $\|w\|_{\mathcal{F} 1}<C_{2}$, with $N, C_{1}, C_{2}$ and the Karamata sequence $b$ as in theorem B.3.9. 
Proof. In a way analogous to the proof of lemma 4.5.21, we deduce

$$
\begin{gathered}
\frac{\partial}{\partial k} \mathcal{A}\left(k, \psi_{-\nu} v, \psi_{\nu} w\right) \\
=-\pi_{K}\left(\begin{array}{cc}
\psi_{-\nu} v & 0 \\
0 & \psi_{\nu} w
\end{array}\right)\left(1-R(0, k, 0,0)\left(1-\pi_{K}\right)\left(\begin{array}{cc}
\psi_{-\nu} v & 0 \\
0 & \psi_{\nu} w
\end{array}\right)\right)^{-1} \\
\cdot R(0, k, 0,0) \mathcal{C} R(0, k, 0,0)\left(1-\pi_{K}\right)\left(\begin{array}{cc}
\psi_{-\nu} v & 0 \\
0 & \psi_{\nu} w
\end{array}\right) \\
\left.\cdot\left(1-R(0, k, 0,0)\left(1-\pi_{K}\right)\left(\begin{array}{cc}
\psi_{-\nu} v & 0 \\
0 & \psi_{\nu} w
\end{array}\right)\right)^{-1}\right|_{K} .
\end{gathered}
$$

Here, $\mathcal{C}$ is the $k$-derivative of $D(k, 0,0)$, which is a bounded operator since the Dirac operator is a first-order differential operator. The lemma now follows due to the weak convergence properties of the expressions involved.

Theorem B.3.13. The functional variation of $\mathcal{A}\left(k, \psi_{-\kappa} v, \psi_{\kappa} w\right)$ with respect to $(v, w)$ around the zero potentials is a nonlinear perturbation of the Fourier transform. More precisely, the variation of $\mathcal{A}$ at $(v, w)=(0,0)$ has the form

$$
(f, g) \mapsto\left(\begin{array}{cc}
\widehat{f}(\nu) & 0 \\
0 & \widehat{g}(-\nu)
\end{array}\right)_{\nu \in \Gamma^{*}} .
$$

In general, the variation has the form

$$
\begin{array}{r}
(f, g) \mapsto \pi_{K}\left(1-\left(\begin{array}{cc}
\psi_{-\nu} v & 0 \\
0 & \psi_{\nu} w
\end{array}\right) R_{r}(k)\left(1-\pi_{K}\right)\right)^{-1}\left(\begin{array}{cc}
\psi_{-\nu} f & 0 \\
0 & \psi_{\nu} g
\end{array}\right) \\
\left.\cdot\left(1-R_{r}(k)\left(1-\pi_{K}\right)\left(\begin{array}{cc}
\psi_{-\nu} v & 0 \\
0 & \psi_{\nu} w
\end{array}\right)\right)^{-1}\right|_{K}
\end{array}
$$

Proof. The general variation can be calculated exactly as in the proof of theorem 4.5.25 (this time, not even a second summand appears in the final result because we did not incorporate the constant parts of $v$ or $w$ into the boundary conditions as we did with $u$ in the Schrödinger case). The Fourier transform property comes from the fact that the zeroth Fourier mode of $\psi_{-\nu} f$ is precisely the $\nu$-th Fourier mode of $f$.

\section{B.3.2 Approximation with finite-type Dirac Fermi curves}

In theorem B.3.9 we found a description of the Fermi curve $F_{D}\left(\psi_{-\nu} v, \psi_{\nu} w\right)$ in $V$, a subset of $\mathbb{C}^{2}$ containing the origin of $\Gamma_{\mathbb{C}}^{*}$ but sufficiently bounded away from the other lattice vectors, which translates to a description of the Fermi curve $F_{D}(v, w)$ near the double points $k_{\nu}^{ \pm} \in \mathbb{C}^{2} / \Gamma^{*}$ by proposition B.2.6. We shall render this description even more explicit by attaching, as in subsection 4.5.2, perturbed Fourier coefficients $\widehat{v}(v, w, \nu)$ and $\widehat{w}(v, w, \nu)$, whose deviation from the unperturbed Fourier coefficients $\widehat{v}(\nu)$ and $\widehat{w}(\nu)$ depends on the size of $v$ and $w$.

However, we shall find ourselves to be unable to prove that these perturbed Fourier coefficients are in the same spaces as the perturbed Fourier coefficients, that is, $\ell^{\infty, 1}\left(\Gamma^{*}\right)$ and $\ell^{1}\left(\Gamma^{*}\right)$, respectively. 
Proposition B.3.14. For all $v_{0} \in \mathcal{F} \ell^{\infty, 1}\left(\Gamma^{*}\right)$ there is a $\delta>0$ such that for all $\nu \in \Gamma_{\delta}^{*}$, all $v \in B_{C_{1}}^{N} \cap \mathcal{F} \ell^{\infty, 1 ; b}\left(\Gamma^{*}\right)$ and all $w \in \mathcal{F} \ell^{1}\left(\Gamma^{*}\right)$ with $\|w\|_{\mathcal{F} 1}<C_{2}$, where the constants $N, C_{1}$ and $C_{2}$ and the Karamata sequence $b$ are chosen as in lemma B.3.12, there is a unique $k_{\nu} \in V$ such that the matrix

$$
\left(\begin{array}{cc}
0 & \pi i k_{\nu, 1}+\pi k_{\nu, 2} \\
-\pi i k_{\nu, 1}+\pi k_{\nu, 2} & 0
\end{array}\right)+\mathcal{A}\left(k_{\nu}, \psi_{-\nu} v, \psi_{\nu} w\right)
$$

is diagonal.

Proof. We shall use the Banach fixed point theorem as in the proof of proposition 4.5.29, only this time, we don't need the implicit function theorem, as all equations involved are linear and hence uniquely solvable.

By lemma B.3.12 we can choose $\delta$ such that for all $\nu \in \Gamma_{\delta}^{*}$ the norm of the $k$-derivative of $\mathcal{A}\left(k, \psi_{-\nu} v, \psi_{\nu} w\right)$ is sufficiently small for an application of the Banach fixed point theorem through the mean value theorem later on. Let $d_{1}(k)$ and $d_{2}(k)$ be the off-diagonal entries of $\mathcal{A}\left(k, \psi_{-\nu} v, \psi_{\nu} w\right)$. Now, let $k \in V$ and $k^{\prime} \in \mathbb{C}^{2}$ and consider the system of equations

$$
\begin{aligned}
\pi i k_{1}^{\prime}+\pi k_{2}^{\prime}+d_{1}(k) & =0, \\
-\pi i k_{1}^{\prime}+\pi k_{2}^{\prime}+d_{2}(k) & =0 .
\end{aligned}
$$

Obviously, this system has a unique solution $k^{\prime}$ for each $k \in V$. Thus, it defines a map

$$
f: V \rightarrow \mathbb{C}^{2}, \quad k \mapsto k^{\prime} .
$$

Now let $k, \widetilde{k} \in V$ be arbitrary. Then $(\overline{B .3 .16})$ implies

$$
\begin{aligned}
\pi i f_{1}(k)+\pi f_{2}(k)+d_{1}(k) & =0, & \pi i f_{1}(\widetilde{k})+\pi f_{2}(\widetilde{k})+d_{1}(\widetilde{k}) & =0, \\
-\pi i f_{1}(k)+\pi f_{2}(k)+d_{2}(k) & =0, & -\pi i f_{1}(\widetilde{k})+\pi f_{2}(\widetilde{k})+d_{2}(\widetilde{k}) & =0,
\end{aligned}
$$

and thus

$$
\begin{aligned}
\pi i f_{1}(k)+\pi f_{2}(k)-\left(\pi i f_{1}(\widetilde{k})+\pi f_{2}(\widetilde{k})\right)+d_{1}(k)-d_{1}(\widetilde{k}) & =0, \\
-\pi i f_{1}(k)+\pi f_{2}(k)-\left(-\pi i f_{1}(\widetilde{k})+\pi f_{2}(\widetilde{k})\right)+d_{2}(k)-d_{2}(\widetilde{k}) & =0 .
\end{aligned}
$$

Now, lemma B.3.12 and the mean value theorem imply for sufficiently small $\delta$ :

$$
\begin{aligned}
& \left\|d_{1}(k)-d_{1}(\widetilde{k})\right\| \leq \epsilon\|k-\widetilde{k}\|, \\
& \left\|d_{2}(k)-d_{2}(\widetilde{k})\right\| \leq \epsilon\|k-\widetilde{k}\|,
\end{aligned}
$$

where $\epsilon>0$ can be chosen arbitrarily small in dependence of $\delta$. The equation system $(\sqrt{B .3 .17})$ can be solved for $f_{1}(k)-f_{1}(\widetilde{k})$ and $f_{2}(k)-f_{2}(\widetilde{k})$ in the same way (B.3.16) can be solved for $k_{1}^{\prime}$ and $k_{2}^{\prime}$, yielding the inequality

$$
\|f(k)-f(\widetilde{k})\| \leq \frac{1}{2}\|k-\widetilde{k}\|
$$

for all sufficiently small $\epsilon$. Domain and codomain of $f$ can be altered to be a closed subset slightly smaller than $V$ by the same reasoning as in proposition 4.5 .29 for sufficiently small $\delta$. Hence, for all $\nu \in \Gamma_{\delta}^{*}$, there is a $k_{\nu} \in V$ such that $f\left(k_{\nu}\right)=k_{\nu}$ by Banach's fixed point theorem. This fixed point precisely fulfils the requirements of (B.3.15). 
Given this proposition, we denote the diagonal entries of $\mathcal{A}\left(k_{\nu}, \psi_{-\nu} v, \psi_{\nu} w\right)$ by $\widehat{v}(v, w, \nu)$ and $\widehat{w}(v, w, \nu)$. In analogy to $\widehat{u}(u, \nu)$, we call them the perturbed Fourier transforms of $v$ and $w$, respectively.

Definition B.3.18. Let $v_{0} \in \mathcal{F} \ell^{\infty, 1}\left(\Gamma^{*}\right)$ and $w_{0} \in \mathcal{F} \ell^{1}\left(\Gamma^{*}\right)$, then we say, in analogy to definition 4.5.32 that the pair of potentials $\left(v_{0}, w_{0}\right)$ has finite type if there are appropriate potentials $v \in \mathcal{F} \ell^{\infty, 1}\left(\Gamma^{*}\right)$ and $w \in \mathcal{F} \ell^{1}\left(\Gamma^{*}\right)$ for which the perturbed Fourier transform is defined for almost all $\nu \in \Gamma^{*}$, such that $F_{D}\left(v_{0}, w_{0}\right)=F_{D}(v, w)$ and for which

$$
\widehat{v}(v, w, \nu)=0 \quad \text { and } \quad \widehat{w}(v, w, \nu)=0
$$

for all but finitely many of those $\nu \in \Gamma^{*}$ for which this condition makes sense.

Recall that, in the above definition, the passing from $F_{D}\left(v_{0}, w_{0}\right)$ to $F_{D}(v, w)$ can be considered a mere technicality since by theorem A.1.6 and proposition B.2.1. it is always possible to switch to a set of potentials covered by previous analysis.

\section{B.3.3 Failure to estimate the perturbed Fourier trans- forms}

At this point, it should be our goal to estimate the perturbed Fourier transforms $(\widehat{v}(v, w, \nu))_{\nu \in \Gamma_{\delta}^{*}}$ and $(\widehat{w}(v, w, \nu))_{\nu \in \Gamma_{\delta}^{*}}$, or, more generally, the (diagonal) entries of $\mathcal{A}\left(k, \psi_{-\nu} v, \psi_{\nu} w\right)$, as we did in the proof of theorem 4.5.42. For this purpose, we should investigate the action of the operator

$$
\left(\begin{array}{cc}
\psi_{-\nu} v & 0 \\
0 & \psi_{\nu} w
\end{array}\right)\left(1-\left(1-\pi_{K}\right) R(0, k, 0,0)\left(\begin{array}{cc}
\psi_{-\nu} v & 0 \\
0 & \psi_{\nu} w
\end{array}\right)\right)^{-1}
$$

on the constant functions. It is easy to see that B.3.19 uniformly maps the Bochner space $\ell^{\infty}\left(\Gamma_{\delta}^{*}, \mathcal{F} \ell^{1}\left(\Gamma^{*}\right)\right) \times \ell^{\infty}\left(\Gamma_{\delta}^{*}, \mathcal{F} \ell^{2,1 ; \sqrt{b}}\left(\Gamma^{*}\right)\right)$ boundedly into the Bochner space $\ell^{\infty}\left(\Gamma_{\delta}^{*}, \mathcal{F} \ell^{\infty, 1 ; b}\left(\Gamma^{*}\right)\right) \times \ell^{\infty}\left(\Gamma_{\delta}^{*}, \mathcal{F} \ell^{2,1 ; \sqrt{b}}\left(\Gamma^{*}\right)\right)$ for suitable $\delta>0$ and Karamata sequence $b$ : consider the operator

$$
\left(1-\pi_{K}\right) R(0, k, 0,0)\left(\begin{array}{cc}
\psi_{-\nu} v & 0 \\
0 & \psi_{\nu} w
\end{array}\right),
$$

use weak convergence properties to invert it for $\nu \in \Gamma_{\delta}^{*}$, then use proposition 2.4.15 to build the full operator (B.3.19). Unfortunately, we can't seem to achieve much more than this rather preliminary result. Let us see what fails to work when we try anyway.

We should work out the diagonal entries of $\mathcal{A}\left(k, \psi_{-\nu} v, \psi_{\nu} w\right)$, that is, the first and the second component, respectively, of the Fourier transform of the operator B.3.19) applied to $\left(\delta_{\kappa 0}, 0\right)^{t}$ and $\left(0, \delta_{\kappa 0}\right)^{t}$, evaluated at $\kappa=0$. For this purpose, we can first expand (B.3.19) as in the proof of theorem 4.5.42

$$
\begin{gathered}
\left(\begin{array}{cc}
\psi_{-\nu} v & 0 \\
0 & \psi_{\nu} w
\end{array}\right)\left(1-\left(1-\pi_{K}\right) R(0, k, 0,0)\left(\begin{array}{cc}
\psi_{-\nu} v & 0 \\
0 & \psi_{\nu} w
\end{array}\right)\right)^{-1} \\
=\left(\begin{array}{cc}
\psi_{-\nu} v & 0 \\
0 & \psi_{\nu} w
\end{array}\right)+\left(\begin{array}{cc}
\psi_{-\nu} v & 0 \\
0 & \psi_{\nu} w
\end{array}\right)\left(1-\pi_{K}\right) R(0, k, 0,0) \\
\cdot\left(\begin{array}{cc}
\psi_{-\nu} v & 0 \\
0 & \psi_{\nu} w
\end{array}\right)\left(1-\left(1-\pi_{K}\right) R(0, k, 0,0)\left(\begin{array}{cc}
\psi_{-\nu} v & 0 \\
0 & \psi_{\nu} w
\end{array}\right)\right)^{-1}
\end{gathered}
$$


The restriction of the first term to $K$ yields

$$
\left(\begin{array}{cc}
\widehat{v}(\nu) & 0 \\
0 & \widehat{w}(-\nu)
\end{array}\right)
$$

which is certainly $\ell^{\infty, 1 ; b}\left(\Gamma_{\delta}^{*}\right)$ and $\ell^{1}\left(\Gamma_{\delta}^{*}\right)$, respectively.

But the second term gives us trouble. Since $\lambda=0$, the Fourier transform of $R(0, k, 0,0)$ only has off-diagonal entries, to wit

$$
\left(\begin{array}{cc}
0 & \frac{1}{\pi i\left(k_{1}+\kappa_{1}\right)-\pi\left(k_{2}+\kappa_{2}\right)} \\
\frac{1}{-\pi i\left(k_{1}+\kappa_{1}\right)-\pi\left(k_{2}+\kappa_{2}\right)} & 0
\end{array}\right)
$$

by B.1.8. Hence, we can write the restricted free resolvent as

$$
\left(1-\pi_{K}\right) R(0, k, 0,0)=\left(\begin{array}{cc}
0 & R_{1}(k) \\
R_{2}(k) & 0
\end{array}\right),
$$

where $R_{1}(k)$ and $R_{2}(k)$ are compact operators from $\mathcal{F} \ell^{2,1 ; \sqrt{b}}\left(\Gamma^{*}\right)$ to $\mathcal{F} \ell^{1}\left(\Gamma^{*}\right)$ and from $\mathcal{F} \ell^{\infty, 1 ; b}\left(\Gamma^{*}\right)$ to $\mathcal{F} \ell^{2,1 ; \sqrt{b}}\left(\Gamma^{*}\right)$, respectively (these operators are, up to sign, simply the restrictions of the inverses of the Wirtinger operators with boundary condition $k$ ). Letting (B.3.19) act on the constant functions, we can define

$$
\begin{aligned}
\left(\begin{array}{l}
f_{1}(k, \nu, x) \\
f_{2}(k, \nu, x)
\end{array}\right):= & \left(\begin{array}{cc}
\psi_{-\nu} v & 0 \\
0 & \psi_{\nu} w
\end{array}\right) \\
& \cdot\left(1-\left(1-\pi_{K}\right) R(0, k, 0,0)\left(\begin{array}{cc}
\psi_{-\nu} v & 0 \\
0 & \psi_{\nu} w
\end{array}\right)\right)^{-1}\left(\begin{array}{l}
1 \\
0
\end{array}\right), \\
\left(\begin{array}{c}
f_{3}(k, \nu, x) \\
f_{4}(k, \nu, x)
\end{array}\right):= & \left(\begin{array}{cc}
\psi_{-\nu} v & 0 \\
0 & \psi_{\nu} w
\end{array}\right) \\
& \cdot\left(1-\left(1-\pi_{K}\right) R(0, k, 0,0)\left(\begin{array}{cc}
\psi_{-\nu} v & 0 \\
0 & \psi_{\nu} w
\end{array}\right)\right)^{-1}\left(\begin{array}{l}
0 \\
1
\end{array}\right) .
\end{aligned}
$$

Considering the expansion (B.3.20), we see the following recursive interdependencies between $f_{1}, \ldots, f_{4}$ :

$$
\begin{array}{ll}
f_{1}=\psi_{-\nu} v+\psi_{-\nu} v R_{1} f_{2}, & f_{3}=\psi_{-\nu} v R_{1} f_{4}, \\
f_{2}=\psi_{\nu} w R_{2} f_{1}, & f_{4}=\psi_{\nu} w+\psi_{\nu} w R_{2} f_{3} .
\end{array}
$$

Let us see what happens when we try to estimate $f_{1}$ and $f_{4}$. We already showed that the first summand of $f_{4}$ yields an $\ell^{1}\left(\Gamma_{\delta}^{*}\right)$-term for the lower diagonal entry of $\mathcal{A}$. To do the same for the second summand, we consider, up to a constant factor, the $\ell^{1}\left(\Gamma^{*}\right)$-norm of the Fourier transform with respect to $x$ (denoted by a hat) at $\kappa=0$. It looks like this:

$$
\sum_{\nu \in \Gamma_{\delta}^{*}}\left|\sum_{\substack{\rho \in \Gamma^{*} \\ \rho \neq 0}} \widehat{w}(-\nu-\rho) \frac{1}{-\pi i\left(k_{1}+\rho_{1}\right)-\pi\left(k_{2}+\rho_{2}\right)} \widehat{f}_{3}(k, \nu, \rho)\right| .
$$

It is possible to make this expression similar to 4.5.44, via

$$
\begin{aligned}
i\left(k_{1}+\rho_{1}\right)+\left(k_{2}+\rho_{2}\right) & =i\left(k_{1}+\rho_{1}+k_{\nu, 1}^{-}\right)+\left(k_{2}+\rho_{2}+k_{\nu, 2}^{-}\right), \\
i\left(k_{1}+\rho_{1}+k_{\nu, 1}^{-}\right)-\left(k_{2}+\rho_{2}+k_{\nu, 2}^{-}\right) & =i\left(k_{1}+\rho_{1}+\nu_{1}\right)-\left(k_{2}+\rho_{2}+\nu_{2}\right) .
\end{aligned}
$$


Hence, we can rewrite the above expression, using the function $g$ defined by 4.5.10 (with sign choice -), by

$$
\sum_{\nu \in \Gamma_{\delta}^{*}}\left|\sum_{\rho \in \Gamma^{*}} \widehat{w}(-\nu-\rho)\left(-\pi i\left(k_{1}+\rho_{1}+\nu_{1}\right)+\pi\left(k_{2}+\rho_{2}+\nu_{2}\right)\right) g(k, \nu, \rho) \widehat{f}_{3}(k, \nu, \rho)\right|,
$$

but by proposition 2.1.6 this is of course equal to

$$
\sum_{\nu \in \Gamma_{\delta}^{*}}\left|\sum_{\rho \in \Gamma^{*}} \widehat{\partial_{k} w}(-\nu-\rho) g(k, \nu, \rho) \widehat{f_{3}}(k, \nu, \rho)\right| .
$$

Now, the potential $w$ being differentiable is not among our prerequisites. With differentiable $w$, with $\partial_{k}$ being in $\mathcal{F} \ell^{1}\left(\Gamma^{*}\right)$, one might think to prove the above expression to be finite with an application of analogs of proposition 4.5.35 and lemmata 4.5 .39 and 4.5 .40 by transforming these statements about $\ell^{\infty, 1}\left(\Gamma^{*}\right)$ sequences to statements about $\ell^{1}\left(\Gamma^{*}\right)$-sequences using the Cauchy condensation theorem. Unfortunately, it turns out that this doesn't work with lemma 4.5.40. It turns out that a straightforward generalisation of these statements works down to $\ell^{2, \infty}$ on the Lorentz scale of spaces, whereas a coarse analysis of the proof of lemma 4.5 .40 shows that it is not applicable for $\ell^{p}$ with $p<2$.

One might seek the reason for this failure in the fact that we assumed very little of $f_{3}$ to begin with. Maybe the situation improves if we first show that $f_{3}$ is from some much smaller space than $\ell^{\infty}\left(\Gamma_{\delta}^{*}, \ell^{\infty, 1}\left(\Gamma^{*}\right)\right)$ ? In order to investigate this avenue of thought, it is instructive to develop $f_{3}$ recursively into its first few terms according to (B.3.21):

$$
\begin{gathered}
\psi_{\nu} w \\
\psi_{\nu} w R_{2} \psi_{-\nu} v R_{1} \psi_{\nu} w \\
\psi_{\nu} w R_{2} \psi_{-\nu} v R_{1} \psi_{\nu} w R_{2} \psi_{-\nu} v R_{1} \psi_{\nu} w
\end{gathered}
$$

While for the first two terms, it is not difficult to show that their Fourier transforms at $\kappa=0$ are in $\ell^{1}\left(\Gamma_{\delta}^{*}\right)$, already in the third term, the convolutions induced by the Fourier transform become so entangled that making an estimate with the usual means appears to be impossible. This is on account of the $\nu$ in $\psi_{ \pm \nu}$ appearing with alternating signs, which in turn is caused by the symmetry established in proposition B.2.6. Loosely put, the influence of $v$ and $w$ to the perturbed Fourier transform mixes badly.

If $v$ and $w$ were from the same space of potentials, that space would necessarily have to be $L^{2}(F)$ or better. In this case, the above problems would not occur (see [Sch02, section 3.5]), and one can legitimately call $\widehat{v}(v, w, \nu)$ the perturbed Fourier transform of $v$, and $\widehat{w}(v, w, \nu)$ the perturbed Fourier transform of $w$, for both $\widehat{v}$ and $\widehat{w}$ are the leading terms of a series expansion of $f_{1}$ and $f_{4}$, respectively. In our case, however, it can be viewed to be somewhat misleading to call $\widehat{w}(v, w, \nu)$ the perturbed Fourier transform of $w$ just because of the leading term when the subsequent terms contain contributions from $v$, which is from a space with much less regular potentials than the space of $w$. Rather, $\widehat{v}(v, w, \nu)$ and $\widehat{w}(v, w, \nu)$ should be viewed as the outcome of the perturbation of $(v, w)$, and unfortunately, they have lost the connexion to the original spaces of $v$ and $w$. 


\section{Bibliography}

[AS61] N. Aronszaijn, K. T. Smith. Theory of Bessel potentials. I. Ann. Inst. Fourier 11 (1961), 385-475.

[Ban22] S. BANACH. Sur les opérations dans les ensembles abstracts et leur application aux équations intégrales. Fund. Math. 3 (1922), 133181.

[Blo72] A. P. Blozinski. On a Convolution Theorem for $L(p, q)$ Spaces. Trans. Amer. Math. Soc. 164 (1972), 255-265.

[Boc33] S. Bochner. Integration von Funktionen, deren Werte die Elemente eines Vektorraumes sind. Fund. Math. 20 (1933), 262-276.

[Boc98] S. V. Bochkarev. The Hausdorff-Young-Riesz theorem in Lorentz spaces and multiplicative inequalities. Proc. Steklov Inst. Math. 219 (1998), 96-107.

[Bor98] É. Borel. Leçons sur la théorie des fonctions. Gauthier-Villars et Fils, Paris, 1898.

[BR80] C. Bennett, K. Rudnick. On Lorentz-Zygmund spaces. Dissertationes Math. 175 (1980), 1-67.

[BS88] C. Bennett, R. Sharpley. Interpolation of Operators, volume 129 of Pure and applied mathematics. Academic Press, Boston, 1988. ISBN 0-12-088730-4.

[Cal61] A. P. CALDERón. Lebesgue spaces of differentiable functions and distributions. In C. B. Morrey, editor, Partial Differential Equations, volume 4 of Proceedings of Symposia in Pure Mathematics. American Mathematical Society, Providence, RI, 1961, 33-49.

[Cal66] A. P. CALderón. Spaces between $L^{1}$ and $L^{\infty}$ and the theorem of Marcinkiewicz. Studia Math. 26 (1966), 273-299.

[CS88] J. H. Conway, N. J. A. Slonne. Sphere Packings, Lattices and Groups, volume 290 of Grundlehren der mathematischen Wissenschaften. Springer-Verlag, New York, 1988. ISBN 0-387-96617$\mathrm{X}$.

[EE04] D. E. Edmunds, W. D. Evans. Hardy Operators, Function Spaces and Embeddings. Monographs in Mathematics. Springer, Heidelberg Berlin, 2004. ISBN 3-540-21972-2. 
[Enf73] P. Enflo. A counterexample to the approximation problem in Banach spaces. Acta Math. 130 (1973), no. 1, 309-317.

[ERT84] G. Eskin, J. Ralston, E. Trubowitz. On Isospectral Periodic Potentials in $\mathbb{R}^{n}$. Commun. Pure Appl. Math. XXXVII (1984), 647676.

[Fis07] E. Fischer. Sur la convergence en moyenne. C. R. Acad. Sci. 144 (1907), 1022-1024.

[FKT90] J. Feldman, H. Knörrer, E. Trubowitz. The perturbatively stable spectrum of a periodic Schrödinger operator. Invent. Math. 100 (1990), 259-300.

[FKT03] J. Feldman, H. Knörrer, E. Trubowitz. Riemann Surfaces of Infinite Genus, volume 20 of Centre de Recherches Mathématiques Université de Montreal Monograph Series. American Mathematical Society, Providence, 2003. ISBN 0-8218-3357-X.

[Fri44] K. O. Friedrichs. The Identity of Weak and Strong Extensions of Differential Operators. Trans. Amer. Math. Soc. 55 (1944), no. 1, $132-151$.

[GGKM67] C. S. Gardner, J. M. Greene, M. D. Kruskal, R. M. Miura. Method for solving the Korteweg-deVries equation. Phys. Rev. Lett. 19 (1967), no. 19, 1095-1097.

[GJ87] J. Glimm, A. Jaffe. Quantum Physics - A Functional Integral Point of View. Springer-Verlag, New York, 2nd edition, 1987. ISBN 0-387-96477-0.

[Gol79] D. Goldberg. A local version of real Hardy spaces. Duke Math. J. 46 (1979), no. 1, 27-42.

[Gro55] A. Grothendieck. Produits tensoriels topologiques et espaces nucléaires. Memoirs of the AMS 1955 (1955), no. 16, 140 pp.

[Hau23] F. HausdorfF. Eine Ausdehnung des Parsevalschen Satzes über Fourierreihen. Math. Zeit. 16 (1923), 163-170.

[Hel12] E. HeLly. Über lineare Funktionaloperationen. Wien. Ber. IIa 121 (1912), 265-297.

[Hen69] D. W. Henderson. Infinite-dimensional manifolds are open subsets of Hilbert space. Bull. Amer. Math. Soc. 75 (1969), 759-762.

[HL30] G. H. Hardy, J. E. Littlewood. A maximal theorem with function-theoretic applications. Acta Math. 54 (1930), 81-116.

[Hö89] O. HÖLDER. Ueber einen Mittelwerthssatz. Gött. Nachr. 1889 (1889), no. 2, 38-47.

[Kat72] T. KATO. Schrödinger operators with singular potentials. Israel J. Math. 13 (1972), no. 1-2, 135-148. 
[Kno64] K. Knopp. Theorie und Anwendung der unendlichen Reihen, volume 2 of Die Grundlehren der mathematischen Wissenschaften in Einzeldarstellungen. Springer, Berlin, 5th edition, 1964. ISBN 3540591117 .

[Kon45] V. I. Kondrashov. Sur certaines propriétés des fonctions dans l'espace $L_{\nu}^{p}$. Dokl. Akad. Nauk SSSR 48 (1945), 535-538.

[Kor08] E. Korotyaev. A priori estimates for the Hill and Dirac operators. Russ. J. Math. Phys. 15 (2008), no. 3, 320-331.

[KP66] S. G. Krein, Y. I. Petunin. Scales of Banach spaces. Russian Math. Surveys 21 (1966), 85-159. Translated by J. L. B. Cooper.

[Kri77] I. M. KRIČEver. Methods of algebraic geometry in the theory of nonlinear equations. Russian Math. Surveys 32 (1977), no. 6, $185-213$.

[KS09] A. Klauer, M. U. Schmidt. Bloch varieties of higherdimensional, periodic Schrödinger operators. J. Appl. Anal. 15 (2009), no. 1, 33-46.

[KT90] H. KNöRRER, E. Trubowitz. A directional compactification of the complex Bloch variety. Comment. Math. Helvetici 65 (1990), $114-149$.

[Kuc93] P. Kuchment. Floquet Theory for Partial Differential Equations, volume 60 of Operator Theory Advances and Applications. Birkhäuser, Basel, 1993. ISBN 3-7643-2901-7.

[Lax55] P. D. LAx. On Cauchy's problem for hyperbolic equations and the differentiability of solutions of elliptic equations. Commun. Pure Appl. Math. 8 (1955), 615-633.

[Leb01] H. Lebesgue. Sur une généralisation de l'intégrale définie. $C$. $R$. Acad. Sci. 132 (1901), 1025-1028.

[Leb02] H. LebesGue. Intégrale, Longueur, Aire. Annali di Mat. serie III, tomo VII (1902), 231-359.

[Lor50] G. G. LoRentz. Some new functional spaces. Ann. Math. 51 (1950), 37-55.

[Lor51] G. G. Lonentz. On the theory of spaces L. Pac. J. Math. 1 (1951), 411-429.

[Lux55] W. A. J. Luxemburg. Banach function spaces. Ph.D. thesis, Technische Hogeschool te Delft, 1955.

[Mar39] J. Marcinkiewicz. Sur l'interpolation d'opérations. C. R. Acad. Sci. 208 (1939), 1272-1272.

[Mar86] V. A. MARČENKO. Sturm-Liouville operators and applications, volume 22 of Operator theory. Birkhäuser, Basel, 1986. ISBN 37643-1794-9. Translated from the Russian by A. Iacob. 
[Min96] H. Minkowski. Geometrie der Zahlen. Lieferung 1. B. G. Teubner, Leipzig, 1896.

[MM75] H. P. McKean, P. van Moerbeke. The spectrum of Hill's equation. Invent. Math. 30 (1975), no. 3, 217-274.

[MO75] V. A. MarČEnko, I. V. OstrovskiI. A characterization of the spectrum of the Hill operator. Mat. Sb. 97 (139) (1975), no. 4 (8), $540-606,633-634$.

[MO80] V. A. Marchenko, I. V. OstrovskiI. Approximation of periodic potentials by finite zone potentials. Vestnik Kharkov. Gos. Univ. 205 (1980), 4-40, 139.

[MR87] J. Mehra, H. Rechenberg. Erwin Schrödinger and the Rise of Wave Mechanics. Part 1: Schrödinger in Vienna and Zurich 18871925, volume 5 of The Historical Development of Quantum Theory. Springer, New York, 1987. ISBN 0-387-95179-2.

[MS64] N. G. Meyers, J. Serrin. $H=W$. Proc. Nat. Acad. Sci. U.S.A. 51 (1964), no. 6, 1055-1056.

[MT76] H. P. McKean, E. Trubowitz. Hill's operator and hyperelliptic function theory in the presence of infinitely many branch points. Commun. Pure Appl. Math. 29 (1976), no. 2, 143-226.

[Nag36] M. NAGumo. Einige analytische Untersuchungen in linearen, metrischen Ringen. Jap. J. Math. 13 (1936), 61-80.

[Nev02] J. S. Neves. Lorentz-Karamata spaces, Bessel and Riesz potentials and embeddings. Dissertationes Math. 405 (2002), 1-46. doi:10. 4064/dm405-0-1.

[NV97] S. P. Novikov, A. P. Veselov. Exactly solvable two-dimensional Schrödinger operators and Laplace transformations. In Solitons, geometry, and topology: on the crossroad, volume 179 of Amer. Math. Soc. Transl. Ser. 2. American Mathematical Society, Providence, RI, 1997, 109-132.

[O'N63] R. O'NeIL. Convolution operators and $L(p, q)$ spaces. Duke Math. J. 30 (1963), 129-142.

[Par06] M.-A. Parseval. Mémoire sur les séries et sur l'intégration complète d'une équation aux différences partielles linéaires du second ordre, à coefficents constants. Mémoires présentés a l'Institut des Sciences, Lettres et Arts, par divers savans, et lus dans ses assemblées 1 (1806), 638-648.

[Pla10] M. Plancherel. Contribution à l'étude de la représentation d'une fonction arbitraire par des intégrales définies. Rend. Circ. Mat. Palermo 30 (1910), 289-335. 
[Ray02] J. W. S. RAYLEIGH. On the character of the complete radiation at a given temperature. In Scientific Papers, volume III, chapter 160. Cambridge University Press, Cambridge, 1902, 268-276. Reprint of Phil. Mag. XXVII (1889), 460-469.

[Rel30] F. ReLlich. Ein Satz über mittlere Konvergenz. Gött. Nachr. 1930 (1930), 30-35.

[Rie07a] F. RiEsz. Sur les systèmes orthogonaux de fonctions. C. R. Acad. Sci. 144 (1907), 615-619.

[Rie07b] F. RiEsz. Ueber orthogonale Funktionensysteme. Gött. Nachr. 1907 (1907), 116-122.

[Rie09] F. Riesz. Sur les suites de fonctions mesurables. C. R. Acad. Sci. 148 (1909), 1303-1305.

[Rie10] F. RIESz. Untersuchungen über Systeme integrierbarer Funktionen. Math. Ann. 69 (1910), 449-497.

[Rie16] F. RIEsz. Über lineare Funktionalgleichungen. Acta Math. 41 (1916), no. 1, 71-98.

[Rie27] M. Riesz. Sur les maxima des formes bilinéaires et sur les fonctionnelles linéaires. Acta Math. 49 (1927), no. 4, 465-497.

[Rog87] L. J. Rogers. An extension of a certain theorem in inequalities. Mess. XVII (1887), no. 2, 145-150.

[RS78] M. Reed, B. Simon. Methods of Modern Mathematical Physics, volume IV: Analysis of Operators. Academic Press, San Diego, 1978. ISBN 0-12-585004-2.

[Sch00] A. Schoenflies. Die Entwickelung der Lehre von den Punktmannigfaltigkeiten. Deutsche Math. Ver. 8 (1900), no. 2, 1-250.

[Sch26a] E. SchröDinger. Quantisierung als Eigenwertproblem. Dritte Mitteilung: Störungstheorie, mit Anwendung auf den Starkeffekt der Balmerlinien. Ann. Phys. 385 (1926), no. 13, 437-490.

[Sch26b] E. SchrÖDINGER. Quantisierung als Eigenwertproblem. Erste Mitteilung. Ann. Phys. 384 (1926), no. 4, 361-376.

[Sch26c] E. SchröDINGER. Quantisierung als Eigenwertproblem. Vierte Mitteilung. Ann. Phys. 386 (1926), no. 18, 109-139.

[Sch26d] E. SchröDinger. Quantisierung als Eigenwertproblem. Zweite Mitteilung. Ann. Phys. 384 (1926), no. 6, 489-527.

[Sch30] J. Schauder. Über lineare, vollstetige Funktionaloperationen. Studia Math. 2 (1930), 183-196.

[Sch50] R. Schatten. A Theory of Cross-Spaces, volume 26 of Annals of Mathematics Studies. Princeton University Press, Princeton, NJ, 1950. ISBN 978-0-691-08396-4. 
[Sch96] M. U. SchmidT. On complex Bloch-spaces of periodic Schrödinger operators, March 1996. Sfb 288 Preprint No. 200, http://www-sfb288.math.tu-berlin.de/Publications/ preprint-list/151/200.

[Sch02] M. U. SchmidT. A proof of the Willmore conjecture, March 2002. Preprint, arXiv:math.DG/0203224v2.

[Sem94] S. SEmmes. A primer on Hardy spaces, and some remarks on a theorem of Evans and Müller. Commun. in Partial Differential Equations 19 (1994), no. 1\&2, 277-319.

[Sha80] R. Sharpley. Counterexamples for classical operators on LorentzZygmund spaces. Studia Math. 68 (1980), no. 2, 141-158.

[Sob63] S. L. Sobolev. On a theorem of functional analysis. In 11 papers on analysis, volume 34 of AMS Transl. (2nd series), 39-68. American Mathematical Society, Providence, RI, 1963. Translated by J. R. Brown from Mat. Sb. (N.S.) 4 (46) (1938), 471-497.

[Ste69] E. M. Stein. Note on the class L log L. Studia Math. 32 (1969), $305-310$.

[Str72] R. S. Strichartz. The Hardy space $H^{1}$ on manifolds and submanifolds. Canad. J. Math. 24 (1972), 915-925.

[Tho39] G. O. Thorin. An extension of the convexity theorem of M. Riesz. Kungl. Fysiogr. Sällsk. Lund Förh. 8 (1939), no. 14. Cited as in Bondesson et al., Life and work of Olof Thorin, Proc. Acad. Sci. Estoniae 57 no. 1 (2008), 18-25.

[Wey12] H. WEYL. Das asymptotische Verteilungsgesetz der Eigenwerte linearer partieller Differentialgleichungen (mit einer Anwendung auf die Theorie der Hohlraumstrahlung). Math. Ann. 71 (1912), 441479 .

[Yos95] K. YosidA. Functional Analysis, volume 123 of Grundlehren der mathematischen Wissenschaften. Springer-Verlag, Berlin, 6th edition, 1995. ISBN 3-540-58654-7.

[You12a] W. H. Young. On the Multiplication of Successions of Fourier Constants. Proc. Roy. Soc. London Ser. A 87 (1912), 331-339.

[You12b] W. H. Young. Sur la généralisation du théorème de Parseval. $C$. R. Acad. Sci. 154 (1912), 30-33.

[You13] W. H. Young. On the determination of the summability of a function by means of its Fourier constants. Proc. London Math. Soc. 12 (1913), 71-88.

[Zyg28] A. Zygmund. Sur les fonctions conjugées. C. R. Acad. Sci. 187 (1928), 1025-1026.

[Zyg29] A. Zygmund. Sur les fonctions conjugées. Fund. Math. 13 (1929), $284-303$. 
[Zyg56] A. Zygmund. On a theorem of Marcinkiewicz concerning interpolation of operations. J. Math. Pures Appl. Ser. IX 35 (1956), $223-248$. 